\title{
Modelos de regressão lineares mistos sob a classe de distribuições normal-potência
}

\author{
Roger Jesús Tovar Falón
}

TESE APRESENTADA

AO

Instituto de Matemática e EstatísticA

DA

Universidade DE SÃo PAUlo

PARA

OBTENÇÃO DO TÍTULO

$\mathrm{DE}$

Doutor EM CiÊnCIAS

\author{
Programa: Estatística \\ Orientador: Prof. Dr. Heleno Bolfarine \\ Coorientador: Prof. Dr. Guillermo Martínez Flórez
}

Durante o desenvolvimento deste trabalho o autor recebeu auxílio financeiro da CAPES e CNPq

São Paulo, Novembro de 2017 


\section{Modelos de regressão lineares mistos sob a classe de distribuições normal-potência}

Esta tese contém as correções e alterações sugeridas

pela Comissão Julgadora durante a defesa realizada por Roger Jesús Tovar Falón em 27/11/2017.

$\mathrm{O}$ original encontra-se disponível no Instituto de Matemática e Estatística da Universidade de São Paulo.

Comissão Julgadora:

- Prof. Dr. Heleno Bolfarine (orientador) - IME-USP

- Prof. Dr. Guillermo Domingo Martínez Flórez - Universidad de Córdoba-Colombia

- Prof. Dr. Aldo William Medina Garay - DE-CCEN-UfPE

- Prof. Dr. Clécio da Silva Ferreira - UFJF

- Prof. Dr. Vicente Garibay Cancho - ICMC-USP 


\section{Agradecimentos}

Em primeiro lugar agradeço a Deus por ter-me acompanhado e guiado em todo momento.

Aos meus pais, Marina e Nicolas, meus irmãos e meus sobrinhos, pelo apoio incondicional em todo momento e as forças que sempre me brindaram.

A Zunny pelo amor e carinho, pela sua paciência, compreensão e pelo apoio durante os dias e momentos de ausência.

A meu orientador o Professor Heleno Bolfarine, pela dedicação, compreensão e confiança em mim depositada, pela orientação que recebi ao longo da elaboração deste trabalho.

A Lina por ter compartido conmigo parte deste tempo, pelo apoio, amor e compreensão durante os momentos difíceis, e pelos grandes momentos vividos durante os últimos anos em São Paulo.

A meu coorientador e amigo, o Professor Guillermo Martínez, em especial pela sua amizade todos estes anos, por ter-me motivado a seguir pelo caminho do trabalho acadêmico e pelas valiosas sugestões ao longo deste trabalho.

A todos os professores da banca examinadora pelas suas sugestões e contribuições importantes para que este trabalho pudesse ser melhorado.

Aos meus amigos e colegas do IME-USP, em especial Elizabeth, Aline e Fabiana, pelos gratos momentos compartilhados.

Ao meu amigo Haroldo Fortes, por ter-me aceito em casa e pela sua amizade.

Aos meus amigos e colegas da Colômbia aqui em São Paulo: Edgardo, Hugo, Mario, Duvan e Edwin, pela sua amizade.

Aos professores do Departamento de Estatística do IME-USP, pelos seus ensinamentos concedidos. 
Aos meus amigos e colegas da Universidad de Córdoba, os professores Carlos Reales e Hugo Aduén, pelo apoio e a confiança depositada em mim para cursar meus estudos de Doutorado.

À Universidade de Córdoba por me dar a oportunidade de vir estudar nesta excelente Universidade.

Finalmente, agradeço o apoio financiero por parte da CAPES e do CNPq. 


\section{Resumo}

Neste trabalho são apresentadas algumas extensões dos modelos potência-alfa assumindo o contexto em que as observações estão censuradas ou limitadas. Inicialmente propomos um novo modelo assimétrico que estende os modelos t-assimétrico (Azzalini e Capitanio, 2003) e $t$-potência (Zhao e Kim, 2016) e inclui a distribuição $t$ de Student como caso particular. Este novo modelo é capaz de ajustar dados com alto grau de assimetria e curtose, ainda maior do que os modelos $t$-assimétrico e $t$-potência. Em seguida estendemos o modelo $t$-potência às situações em que os dados apresentam censura, com alto grau de assimetria e caudas pesadas. Este modelo generaliza o modelo de regressão linear $t$ de Student para dados censurados por Arellano-Valle et al. (2012)

O trabalho também introduz o modelo linear misto normal-potência para dados assimétricos. Aqui a inferência estatística é realizada desde uma perspectiva clássica usando o método de máxima verossimilhança junto com o método de integração numérica de Gauss-Hermite para aproximar as integrais envolvidas na função de verossimilhança. Mais tarde, o modelo linear com interceptos aleatórios para dados duplamente censurados é estudado. Este modelo é desenvolvido sob a suposição de que os erros e os efeitos aleatórios seguem distribuições normal-potência e normal-assimétrica. Para todos os modelos estudados foram realizados estudos de simulação a fim de estudar as suas bondades de ajuste e limitações. Finalmente, ilustram-se todos os métodos propostos com dados reais.

Palavras-chave: modelos lineares mistos, distribuição normal-potência, distribuição normalassimétrica, estimação por máxima verossimilhança, quadratura de Gauss-Hermite. 


\section{Abstract}

In this work some extensions of the alpha-power models are presented, assuming the context in which the observations are censored or limited. Initially we propose a new asymmetric model that extends the skew- $t$ (Azzalini e Capitanio, 2003) and power- $t$ (Zhao e Kim, 2016) models and includes the Student's $t$-distribution as a particular case. This new model is able to adjust data with a high degree of asymmetry and cursose, even higher than the skew- $t$ and power- $t$ models. Then we extend the power- $t$ model to situations in which the data present censorship, with a high degree of asymmetry and heavy tails. This model generalizes the Student's $t$ linear censored regression model $t$ by Arellano-Valle et al. (2012)

The work also introduces the power-normal linear mixed model for asymmetric data. Here statistical inference is performed from a classical perspective using the maximum likelihood method together with the Gauss-Hermite numerical integration method to approximate the integrals involved in the likelihood function. Later, the linear model with random intercepts for doubly censored data is studied. This model is developed under the assumption that errors and random effects follow power-normal and skew-normal distributions. For all the models studied, simulation studies were carried out to study their benefits and limitations. Finally, all proposed methods with real data are illustrated.

Keywords: linear mixed models, power-normal distribution, skew-normal distribution, maximum likelihood estimation, Gauss-Hermite quadrature. 


\section{Sumário}

Lista de Figuras $\quad$ viii

Lista de Tabelas $\quad$ x

1 Introdução 1

2 Preliminares 3

2.1 Truncamento e Censura . . . . . . . . . . . . . . . . . . . . 3

2.1 .1 Truncamento . . . . . . . . . . . . . . . . 3

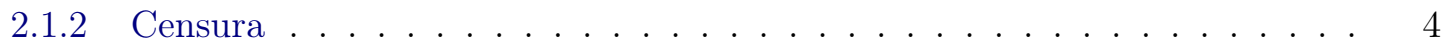

2.2 Modelo Linear Normal de Efeitos Mistos . . . . . . . . . . . . . . . . . 4

2.2.1 Abordagem Marginal . . . . . . . . . . . . . . . . . . 5

2.2 .2 Abordagem Condicional . . . . . . . . . . . . . . . 5

2.3 Distribuições Normal-Assimétrica e $t$-Assimétrica . . . . . . . . . . . . . 6

2.3.1 Distribuição Normal-Assimétrica . . . . . . . . . . . . . . . . 6

2.3.2 Distribuição Normal-Assimétrica Truncada . . . . . . . . . . . . . 7

2.3 .3 Distribuição t-Assimétrica . . . . . . . . . . . . . . . . . . . 8

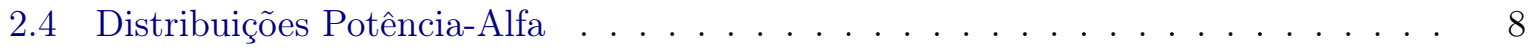

2.4.1 Distribuição Normal-Potência . . . . . . . . . . . . . . . . . . 9

2.4.2 Distribuição t-Potência . . . . . . . . . . . . . . . . . . . . . 10

2.4.3 Momentos nos Modelos Normal-Potência e t-Potência . . . . . . . . . . . 10

2.4.4 Distribuição Normal-Potência Multivariada . . . . . . . . . . . . . . . . . 12

2.5 Quadratura de Gauss-Hermite . . . . . . . . . . . . . . . . . . . . 14

2.5.1 Quadratura de Gauss-Hermite Unidimensional . . . . . . . . . . . . . 15

2.5.2 Quadratura de Gauss-Hermite Multidimensional . . . . . . . . . . . . 15

3 Extensões do Modelo t-Potência $\quad 16$

3.1 Introdução . . . . . . . . . . . . . . . . . . . . . . . 16

3.2 Modelo t-Assimétrico-Potência . . . . . . . . . . . . . . . 17

$3.2 .1 \quad$ Estimação . . . . . . . . . . . . . . . . . . . . . . . . . . 18

3.2 .2 Matriz de Informação . . . . . . . . . . . . . . . . . . . . . . . . . . . . . . . . . . . . . . . . . . . . . . . . .

3.2 .3 Modelo PST de Locação e Escala . . . . . . . . . . . . . . . . . . . . . . . 21

3.3 Aplicação . . . . . . . . . . . . . . . . . . . . 25 
3.4 Modelo $t$-Potência para Dados Censurados e Truncados . . . . . . . . . . . . 28

3.4.1 O Modelo t-Potência para Dados Censurados . . . . . . . . . . . . . . 28

3.4.2 O Modelo t-Potência para Dados Truncados . . . . . . . . . . . . . . . 30

3.5 O Modelo de Regressão Linear t-Potência Censurado . . . . . . . . . . . . . . . . 31

3.5.1 Momentos . . . . . . . . . . . . . . . . . . . . . 32

3.5 .2 Estimação . . . . . . . . . . . . . . . . . . . . . . . . 33

3.6 Seleção de Modelos e Análise de Resíduos . . . . . . . . . . . . . . . . . . . . . . 35

3.6.1 Seleção de Modelos . . . . . . . . . . . . . . . . . . . . . . . . . . . . 35

3.6 .2 Análise de resíduos . . . . . . . . . . . . . . . . . . . . . . 35

3.7 Simulação . . . . . . . . . . . . . . . . . . . . . . . . . 36

3.8 Aplicação . . . . . . . . . . . . . . . . . . . . . . . . 36

3.9 Conclusões . . . . . . . . . . . . . . . . . . . . . . . . . . 39

4 Modelos Lineares Mistos Normal-Potência 41

4.1 Introdução . . . . . . . . . . . . . . . . . . . . . . . . . . . . . . . 41

4.2 O Modelo com Interceptos Aleatórios Normal-Potência . . . . . . . . . . . . . . . 42

4.2 .1 Momentos . . . . . . . . . . . . . . . . . . . . . . . . . 42

$4.2 .2 \quad$ Estimação . . . . . . . . . . . . . . . . . . . . . . . . . . . 44

4.2 .3 Predição de efeitos aleatórios . . . . . . . . . . . . . . . . . . 46

4.2 .4 Seleção de modelos . . . . . . . . . . . . . . . . . . . . . . . . . . . 46

4.2 .5 Análise de resíduos . . . . . . . . . . . . . . . . . . . . . . . 46

4.3 O Modelo Linear Misto Normal-Potência . . . . . . . . . . . . . . . . . . . . . 48

4.3 .1 Momentos . . . . . . . . . . . . . . . . . . . . . . . 49

4.3 .2 Estimação . . . . . . . . . . . . . . . . . . . . . . . . . . 50

4.4 Simulação . . . . . . . . . . . . . . . . . . . . . . . . . . . . 51

4.5 Aplicação . . . . . . . . . . . . . . . . . . . . . . . . . 52

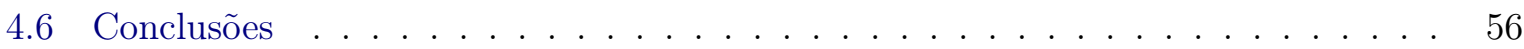

5 Modelos de Regressão Lineares Mistos Assimétricos para Dados Duplamente Censurados $\quad 57$

5.1 Introdução . . . . . . . . . . . . . . . . . . . . . . . . . 57

5.2 Modelo de Regressão Duplamente Censurado com Efeitos Aleatórios . . . . . . . 57

5.2 .1 Momentos . . . . . . . . . . . . . . . . . . . . . . . . 59

5.2 .2 Estimação . . . . . . . . . . . . . . . . . . . . . . . . . 60

5.3 Modelo PNDC-RE . . . . . . . . . . . . . . . . . . . . . 60

5.3.1 Momentos no Modelo PNDC-RE . . . . . . . . . . . . . . . . . . . 60

5.3 .2 Estimação no Modelo PNDC-RE . . . . . . . . . . . . . . . . . . . . . 61

5.3 .3 Modelo PNT-RE . . . . . . . . . . . . . . . . . . . . . . . 63

5.4 Modelo SNDC-RE . . . . . . . . . . . . . . . . . . . . . . 66

5.4.1 Momentos no Modelo SNDC-RE . . . . . . . . . . . . . . . . . 67

5.4 .2 Estimação no Modelo SNDC-RE . . . . . . . . . . . . . . . . . . . . . 68 
5.4 .3 Modelo SNT-RE . . . . . . . . . . . . . . . . . . . . . . . . . 69

5.5 Seleção de modelos . . . . . . . . . . . . . . . . . . . . . . . 72

5.6 Simulação . . . . . . . . . . . . . . . . . . . . . . . . . . . 72

5.7 Aplicações . . . . . . . . . . . . . . . . . . . . . . . . . . . . . . . . 73

5.7.1 Dados de Doença Periodontal . . . . . . . . . . . . . . . . . . . . 73

5.7 .2 Dados de Colesterol . . . . . . . . . . . . . . . . . . . . . . 75

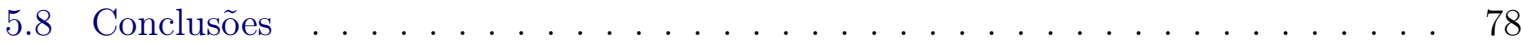

6 Conclusões Finais e Perspectivas Futuras $\quad 81$

6.1 Conclusões Finais . . . . . . . . . . . . . . . . . . . . . . . . . . . . . . . 81

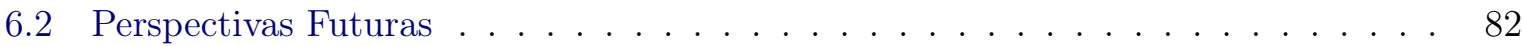

A Tabelas das Simulações $\quad 83$

B Funções Escore e Matrizes de Informação. $\quad 88$

B.1 Matriz de Informação para o Modelo PST . . . . . . . . . . . . . . . . . . . 88

B.1.1 Modelo PST Padronizado . . . . . . . . . . . . . . . . . . . . . . . 88

B.1.2 Modelo PST de Locação-Escala . . . . . . . . . . . . . . . . . . . . . . . . 91

B.2 Matriz de Informação Observada: Modelo PT Censurado . . . . . . . . . . . . . . 98

B.3 Matriz de Informação Observada: Modelo PT Truncado . . . . . . . . . . . . . . 100

B.4 Matriz de Informação Modelo PT-CR . . . . . . . . . . . . . . . . . . . . . . . 103

B.4.1 Matriz de Informação Observada . . . . . . . . . . . . . . . . . . . . 103

B.4.2 Matriz de Informação Esperada . . . . . . . . . . . . . . . . . . . . . . . 105

B.5 Função Escore e Matriz de Informação Observada no Modelo PNLMM . . . . . . 108

B.5.1 Função Escore . . . . . . . . . . . . . . . . . . . . . . . . . . . . . . . 108

B.5.2 Matriz de Informação Observada . . . . . . . . . . . . . . . . . . . . . . 109

B.5.3 Função Escore para o PNLMM . . . . . . . . . . . . . . . . . . . . . . . . 112

B.6 Função Escore e Matriz de Informação Observada para o Modelo PNDC-RE . . . 113

B.6.1 Função Escore . . . . . . . . . . . . . . . . . . . . . . . . . . . . . . . . . 114

B.6.2 Matriz de Informação Observada . . . . . . . . . . . . . . . . . . . . . . 114

Referências Bibliográficas $\quad 120$ 


\section{Lista de Figuras}

2.1 Função densidade de probabilidade da $\operatorname{SN}(\lambda)$ para $\lambda=-3$ (linha sólida), $\lambda=0$ (linha tracejada), $\lambda=1$ (linha pontilhada), $\lambda=3$ (linha pontilhada-tracejada) e $\lambda=5$ (linha longa-tracejada) . . . . . . . . . . . . . . .

2.2 Função densidade de probabilidade de: (a) Distribuição $\operatorname{PN}(\alpha)$ para $\alpha=0.25$ (linha sólida), $\alpha=0.50$ (linha tracejada), $\alpha=1.0$ (linha pontilhada), $\alpha=1.5$ (linha pontilhada-tracejada) e $\alpha=3.5$ (linha longa-tracejada). (b) Distribuição $\operatorname{PT}(\alpha, \nu=5)$ para $\alpha=0.25$ (linha sólida), $\alpha=0.50$ (linha tracejada), $\alpha=1.0$ (linha pontilhada), $\alpha=1.5$ (linha pontilhada-tracejada) e $\alpha=3.5$ (linha longatracejada) . . . . . . . . . . . . . . . . . .

2.3 Contornos da distribuição normal-potência 2-variada para of $\boldsymbol{\alpha}=\left(\alpha_{1}, \alpha_{2}, \alpha\right)^{\top}$ : (a) $(0.5,0.5,0.5)$, (b) $(0.10,100,100),(c)(0.20,0.20,2.0)$ e (d) $(1.0,1.0,100)$.

3.1 Função densidade de probabilidade de: (a) Distribuição $\operatorname{PST}(\lambda, 2.5,6)$ para $\lambda=$ -2.0 (linha sólida), $\lambda=-1.0$ (linha tracejada), $\lambda=0.5$ (linha pontilhada), $\lambda=2.5$ (linha pontilhada-tracejada). (b) Distribuição $\operatorname{PST}(-1, \alpha, 6)$ para $\alpha=0.5$ (linha sólida), $\alpha=0.75$ (linha tracejada), $\alpha=1.5$ (linha pontilhada), $\alpha=4$ (linha

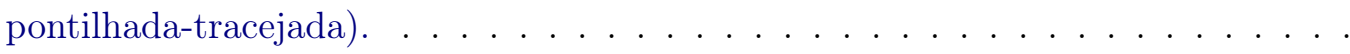

3.2 Perfil da função de log-verossimilhança $\ell_{p}(\alpha)$ (primeira linha), $\ell_{p}(\lambda)$ (segunda linha) e $\ell_{p}(\nu)$ (terceira linha). Tamanhos de amostras $n=50$ (primeira coluna), $n=100$ (segunda coluna), e $n=200$ (terceira coluna).

3.3 Envelopes dos resíduos quantis aleatorizados para (a) Modelo T, (b) Modelo ST, (c) Model PT, (d) Modelo PST. . . . . . . . . . . . . . . . . . . . . . .

3.4 (a) Gráfico dos modelos ajustados aos dados de vulcões. PST (linha sólida), T (linha tracejada), PS (linha pontilhada) e ST (linha pontilhada-tracejada). (b) fda empírica de $Y$ e fda de modelo PST ajustado. . . . . . . . . . . . . . 28

3.5 Histograma dos Salários, dados de Mroz. . . . . . . . . . . . . . . . . . . . 37

3.6 Envelopes dos resíduos tipo martingais transformados $r_{M T i}$, para (a) Modelo PTCR. (b) Modelo T-CR. (c) Model SL-CR. (d) Modelo N-CR. . . . . . . . . . . .

4.1 Dados de colesterol: (a) Histograma do nível de colesterol para 200 sujeitos do estudo de Framingham. (b) Trajetórias para cinco sujeitos aleatóriamente selecionados. 
4.2 Gráfico de probabilidade meio-normal com envelope simulado para o modelo PNPN (a), modelo N-PN (b), modelo N-SN (c), e modelo N-N (d) . . . . . . . . . . . 55

5.1 Histograma para os dados de doença periodontal. Os "pinos"nos extremos representam a proporção de observações censuradas $(9,8 \%)$ à esquerda e $(8,1 \%)$ à direita. 74

5.2 Histograma para os dados de Colesterol. O "pino"no extremo inferior representa a frequência de observações censuradas (108) no valor $c_{0}=1.82 \ldots$. . . . . . . . 77 


\section{Lista de Tabelas}

3.1 Intervalo de valores para os coeficientes de assimetria e curtose dos modelos $\operatorname{ST}(\lambda, \nu), \operatorname{PT}(\alpha, \nu)$ e $\operatorname{PST}(\lambda, \alpha, \nu)$ baseados num estudo de simulação. Valores de $\lambda$ de -40 a $40, \alpha$ de 0.5 a 50 , e $\nu=2,3,4,5,6,7 \ldots \ldots \ldots \ldots$

3.2 Resumo estatístico para os dados de vulcões. . . . . . . . . . . . . . 26

3.3 Estimativas dos parâmetros e erros padrão (EP) para os modelos T, ST, PT e PST ajustados aos dados de vulcões. . . . . . . . . . . . . . . 26

3.4 Critérios de seleção dos modelos ajustados aos dados de vulcões. . . . . . . . . 26

3.5 Resumo estatístico para os dados de salários. . . . . . . . . . . . . . . 37

3.6 Estimativas de MV dos parâmetros e erros padrões (EP) obtidos no ajuste dos modelos PT-CR, T-CR, SL-CR e N-CR para os dados de salários. . . . . . . . . 38

3.7 Critérios de seleção dos modelos ajustados aos dados de salários. . . . . . . . . . 38

4.1 Resumo estatístico para os dados de colesterol. . . . . . . . . . . . . . . . 53

4.2 Estimativas de MV dos parâmetros e erros padrões (EP) obtidos no ajuste dos modelos PNPN, NPN, NSN, e N para os dados de colesterol. . . . . . . . . . . 54

4.3 Critérios de seleção dos modelos ajustados aos dados de colesterol. . . . . . . . . . 54

5.1 Estimativas de MV dos parâmetros e erros padrões (EP) obtidos no ajuste dos modelos DCN-PN, DCPN-N, DCN-SN, DCSN-N e DCN-N aos dados de doença periodontal. . . . . . . . . . . . . . . . . 76

5.2 Critérios de seleção dos modelos ajustados aos dados de doença periodontal. . . . 77

5.3 Estimativas de MV dos parâmetros e erros padrões (EP) obtidos no ajuste dos modelos DCN-PN, DCPN-N, DCN-SN, DCSN-N e DCN-N aos dados de colesterol. 79

5.4 Critérios de seleção dos modelos ajustados aos dados de colesterol. . . . . . . . . 80

A.1 Avaliação do desempenho dos estimadores de máxima verossimilhança de $\beta_{0}, \beta_{1}$, $\eta, \alpha$ e $\nu$ no modelo PT-CR. . . . . . . . . . . . . . . . . 84

A.2 Avaliação do desempenho dos estimadores de máxima verossimilhança de $\beta_{0}, \beta_{1}$, $\eta_{e}, \eta_{g}, \alpha_{e}$ e $\alpha_{g}$ no modelo com interceptos aleatórios normal-potência. . . . . . . 85

A.3 Avaliação do desempenho dos estimadores de máxima verossimilhança de $\beta_{0}, \beta_{1}$, $\eta_{e}, \eta_{g}, \alpha_{e}$ e $\alpha_{g}$ no modelo PNDC-RE. . . . . . . . . . . . . . 86 
A.4 Avaliação do desempenho dos estimadores de máxima verossimilhança de $\beta_{0}, \beta_{1}$,

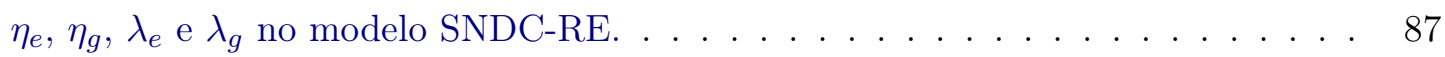




\section{Capítulo 1}

\section{Introdução}

Modelos lineares mistos (MLM's) tornaram-se bastante populares e são frequentemente usados na análise de medidas repetidas contínuas a partir de uma amostra de unidades em várias áreas do conhecimento, tais como agricultura, biologia, ensaios clínicos, pesquisas médicas e estudos de meio ambiente (veja por exemplo, Diggle et al. (2004), McCulloch et al. (2008) e Verbeke e Molenberghs (2009)). Estes modelos também têm sido estudados para análise de dados discretos (Molenberghs e Verbeke, 2005). Usualmente no ajuste dos modelos lineares mistos os efeitos aleatórios e o termo de erro são considerados ter uma distribuição normal; mas esta suposição pode não ser adequada e pode levar a consequências danosas para a inferência. Por exemplo, Vock et al. (2012) mostram que em geral, os estimadores de máxima verossimilhança não são consistentes quando a função densidade de probabilidade para os efeitos aleatórios é incorretamente especificada, e este efeito pode ser maior quanto mais a verdadeira densidade dos efeitos aleatórios desvia-se da densidade normal.

Uma complicação adicional na utilização dos modelos mistos para modelagem de dados longitudinais surge quando a resposta estudada é limitada ou censurada. Por exemplo, nos ensaios clínicos, a variável resposta pode tomar valores limites para uma parte dos dados; estes valores são chamados limites de detecção mínimo $(\mathrm{LDm})$ quando temos truncamento à esquerda (como considerado em Martínez-Flórez et al. (2013b)) e máximo (LDM) quando temos truncamento à direita. Alguns trabalhos desenvolvidos neste contexto são: Hughes (1999), Jacqmin-Gadda et al. (2000), Pan e Louis (2000) e Wu (2002) entre outros).

Apesar de que uma variedade de métodos têm sido propostos para flexibilizar a suposição de normalidade dos efeitos aleatórios no uso dos modelos lineares mistos (Verbeke e Lasaffre (1997), Aitkin (1999), Chen et al. (2002) e Lee e Thompson (2008)), existe pouca literatura em que estes métodos têm sido implementados para dados longitudinais limitados ou censurados. Uma proposta de estudo interessante é considerar distribuições mais flexíveis para os efeitos aleatórios como a família de distribuições potência normal (Gupta e Gupta, 2008), as quais têm mostrado ser capazes de modelar o grau de variabilidade do viés e a curtose num conjunto de dados.

Embora os modelos mistos com respostas limitadas tem recebido considerável atenção na literatura recente, a grande maioria propõem a distribuição beta (no caso de proporções) para a modelagem da resposta; entre outros mencionamos Ferrari e Cribari-Neto (2004), Branscum et al. (2007), Bayes et al. (2012) e mais recentemente Galvis et al. (2014); uma alternativa a considerar para este tipo de dados é o modelo tobit modificado (Martínez-Flórez et al., 2013b) que é uma extensão do modelo tobit (Tobin, 1958) o qual, em situações práticas em que os dados apresentam assimetria e curtose maior do que é esperado para o modelo normal, pode ser mais preciso que o modelo de regressão beta usual.

A abordagem Bayesiana também têm sido proposta para a inferência estatística nos modelos mistos (Branscum et al. (2007), Fong et al. (2010) e Figueroa-Zúñiga et al. (2013), e para 
dados duplamente censurados como em Yu (2010) e Galvis et al. (2014)). Esta abordagem, contudo, tem sido prejudicada pela falta de implementação rápida e a dificuldade na especificação das distribuições a priori, além de ter que considerar prioris para os componentes de variância, que são particularmente problemáticos.

Um cenário interessante de estudo na literatura é portanto, considerar modelos mistos censurados com distribuições da classe potência normal para os erros e os efeitos aleatórios. Apresentaremos neste trabalho, um estudo dos modelos lineares mistos com respostas limitadas e censuradas, isto com o objetivo de preencher esta lacuna e propor alternativas de modelagem deste tipo de dados. Para a inferência dos modelos serão consideradas as abordagem clássica.

\section{Organização do trabalho}

Este trabalho tem a seguinte estrutura. O Capítulo 2 é um capítulo introdutório, que apresenta alguns conceitos de grande importância no desenvolvimento deste trabalho. Faz-se uma revisão do modelo linear misto introduzido por Laird e Ware (1982) e da famímila de distribuições potência-alfa junto com as principais características dessas distribuições. A distribuição normal-assimétrica e $t$-assimétrica são também apresentadas. Finalmente o método de integração numérica de Gauss-Hermite nas versões unidimensional e multidimensional é apresentada.

No Capítulo 3 fazemos extensões do modelo $t$-potência introduzido por Zhao e Kim (2016). Inicialmente introduzimos o modelo $t$-assimétrico-potência para a modelagem de dados com presença de assimetria e curtose. Para este modelo usamos o método de máxima verossimilhança para fazer a inferência dos parâmetros envolvidos no modelo. São apresentados os elementos da função escore bem como os elementos da matriz de informação observada. Uma aplicação a dados reais para mostrar o uso de modelo é também apresentada. Em seguida, o modelo de regressão $t$-potência censurado (PT-CR) é apresentado. O modelo PT-CR é útil para as situações em que os dados apresentam grau de assimetria e curtose maior do que o modelo normal usual. Apresentamos a metodologia de estimação dos parâmetros e uma simulação é realizada para estudar as bondades de ajuste e limitações do modelo. Por fim uma aplicação a dados reais é realizada.

O Capítulo 4 introduz o modelo de regressão linear normal-potência com interceptos aleatórios normal-potência. Derivamos os dois primeiros momentos da variável resposta e propomos um método de estimação dos parâmetros do modelo. Uma metodologia para obter as predições dos interceptos aleatórios é proposta. Derivamos também a função escore aproximada e os elementos aproximados da matriz de informação observada. A extensão do modelo ao caso multivariado é também considerada neste capítulo. Para estudar as bondades dos estimadores de máxima verossimilhança realizamos uma simulação de Monte Carlo e finalmente uma aplicação a datos de colesterol é realizada para ilustrar a proposta.

Finalmente, no Capítulo 5 fazemos a extensão do modelo linear normal-potência com interceptos aleatórios normal-potência para as situasões em que os dados (assimétricos e de caudas pesadas) apresentam censura ou estão limitados (duplamente censurados). São introduzidos dois novos modelos os quais são baseados nas distribuições normal-potência e normal-assimétrica. Para ambos os modelos derivamos os dois primeiros momentos e propomos uma metodologia de estimação dos parâmetros. Propomos também alguns critérios para a seleção dos modelos e uma simulação para estudar as propriedades assintóticas dos estimadores de máxima verossimilhança é realizada. Por fim, uma aplicação com dados reais é apresentada. 


\section{Capítulo 2}

\section{Preliminares}

Ao longo deste trabalho utilizaremos algumas notações as quais serão inicialmente denotadas neste capítulo.

Sendo assim, seja $X \sim \mathrm{N}\left(\mu, \sigma^{2}\right)$ uma variável aleatória seguindo uma distribuição normal com média $\mu$ e variância $\sigma^{2}$, e $\phi\left(\cdot \mid \mu, \sigma^{2}\right)$ denota a sua função densidade de probabilidade (fdp). $\phi(\cdot)$ e $\Phi(\cdot)$ denotam respectivamente a fdp e a função de distribuição acumulada (fda) da normal padrão, isto é, $\mu=0$ e $\sigma^{2}=1$.

Similarmente $Y \sim \mathrm{T}(\mu, \sigma, \nu)$ uma variável aleatória seguindo uma distribuição $t$ de Student com parâmetro de locação $\mu$, parâmetro de escala $\sigma$ e graus de liberdade $\nu>0$, e $t(\cdot \mid \mu, \sigma, \nu)$ denota a sua fdp. $t(\cdot \mid \nu)$ e $T(\cdot \mid \nu)$ denotam respectivamente a fdp e a fda da $t$ de Student padrão com graus de liberdade $\nu$, isto é, $\mu=0, \sigma=1 \mathrm{e}$

$$
t(z \mid \nu)=\frac{\Gamma\left(\frac{\nu+1}{2}\right)}{\sqrt{\pi \nu} \Gamma\left(\frac{\nu}{2}\right)}\left\{1+\frac{z^{2}}{\nu}\right\}^{-\frac{\nu+1}{2}},
$$

e

$$
T(z \mid \nu)=\int_{-\infty}^{z} t(s \mid \nu) d s .
$$

Em geral, utilizamos a convenção tradicional denotado uma variável aleatória (ou um vetor aleatório) por uma letra maiúscula e sua realização pela letra minúscula correspondente. Vetores aleatórios e matrizes são representados por letras em negrito. $\mathbf{X}^{\top}$ é a transposição de $\mathbf{X}$.

\subsection{Truncamento e Censura}

Neste trabalho estudaremos alguns modelos regressão quando a variável resposta apresenta observações incompleta devido a censura. Seguindo Garay (2014) esclarecemos a diferença entre as duas principais causas de dados incompletos: a censura e o truncamento os quais serão utilizados ao longo de nosso texto.

\subsubsection{Truncamento}

O truncamento ocorre quando algumas observações, tanto na variável resposta como nas variáveis regressoras não estão disponíveis, isto é, o truncamento é caracterizado por uma condição que exclui certos indivíduos do estudo. Bem como Garay (2014) apresentamos o exemplo dado por Colosimo e Giolo (2006), em que o truncamento ocorre se, para a estimação da distribuição do tempo de vida dos moradores de uma certa localidade, for usada uma amostra retirada do banco de dados da previdência local. Desta forma, somente moradores que atingiram 
a aposentadoria fazem parte da amostra. Assim, estas observações são conhecidas por truncadas à esquerda. Outros exemplos de truncamentos podem ser encontrados em Nelson (1990) e Kalbfleish e Lawless (1992).

\subsubsection{Censura}

A censura ocorre quando dados sobre a variável resposta não estão completamente disponíveis para algumas unidades da amostra, no entanto para estas unidades, os dados sobre as variáveis regressoras são totalmente conhecidos. Em varias situações práticas, a censura acontece por razões tais como as limitações dos equipamentos de medição ou do projeto experimental. Assim, informalmente podemos dizer que uma observação censurada contém apenas informações parciais sobre um evento de interesse. Por exemplo a agulha de uma escala que não fornece uma leitura acima de $200 \mathrm{~kg}$ para todos os objetos que pesam mais do que o limite.

Modelos emvolvendo respostas censuradas com erros de observação normalmente distribuídos têm sido estudados extensivamente na literatura (veja por exemplo Nelson (1977); Park et al. (2007); Stapleton e Young (1984); Thompson e Nelson (2003) e Vaida e Liu (2009)).

Em modelos de regressão linear censurados com distibuição $t$ de Student, os traballhos de Arellano-Valle et al. (2012) e Matos et al. (2013) podem ser mencionados, a partir de uma abordagem clássica. Finalmente, modelos com respostas com erros de observação com uma classe de distribuição com caudas mais pesadas do que a normal, a partir de uma abordagem clássica e Bayesiana foram introduzidos por Garay et al. (2017).

\subsection{Modelo Linear Normal de Efeitos Mistos}

Um de nossos objetivos neste trabalho é estudar modelos de regressão mistos quando a variável resposta apresenta censura (à esquerda, direita ou dupla censura). Assim, descreveremos brevemente o modelo linear de efeitos mistos introduzido por Laird e Ware (1982).

O modelo linear de efeitos mistos introduzido por Laird e Ware (1982) é dado por

$$
\mathbf{Y}_{i}=\mathbf{X}_{i} \boldsymbol{\beta}+\mathbf{Z}_{i} \gamma_{i}+\boldsymbol{\epsilon}_{i}, \quad i=1, \ldots, n,
$$

em que $\mathbf{Y}_{i}=\left(Y_{i 1}, \ldots, Y_{i m_{i}}\right)^{\top}$ é o vetor de dimensão $m_{i} \times 1$ que contém as respostas correspondentes ao $i$-ésimo indivíduo para $i=1, \ldots, n ; \boldsymbol{\beta}=\left(\beta_{1}, \ldots, \beta_{p}\right)^{\top}$ de dimensão $p \times 1$ é o vetor de parâmetros de efeitos fixos; $\mathbf{X}_{i}$ é uma matriz de planejamento de dimensão $m_{i} \times p$ correspondente ao vetor de parâmetros de efeitos fixos $\boldsymbol{\beta}$; e $\mathbf{Z}_{i}$ é matriz de planejamento de dimensão $m_{i} \times q$, correspondente ao vetor de efeitos aleatórios $\gamma_{i}=\left(\gamma_{i 1}, \ldots, \gamma_{i q}\right)^{\top}$ de dimensão $q \times 1$ em que $\gamma_{i}$ segue distribuição $\gamma_{i} \sim \mathrm{N}_{q}(\mathbf{0}, \mathbf{D})$. O vetor de erros $\boldsymbol{\epsilon}_{i}=\left(\epsilon_{i 1}, \ldots, \epsilon_{i m_{i}}\right)^{\top}$ de dimensão $m_{i} \times 1$ segue uma distribuição normal com vetor de média zero e matriz de variância-covariância $\mathbf{R}_{i}$, isto é $\boldsymbol{\epsilon}_{i} \sim \mathrm{N}_{m_{i}}\left(\mathbf{0}, \mathbf{R}_{\mathbf{i}}\right)$.

A inferência para o modelo (2.3) pode ser feita usando abordagem marginal em que os $\gamma_{i}$ 's são considerados como uma amostra aleatória independente da distribuição $\mathrm{N}_{q}(\mathbf{0}, \mathbf{D})$ e é apropriada quando estamos interessados nos coeficientes específicos para o indivíduo $i$ (veja Verbeke e Molenberghs, 2009). Outra abordagem é a condicional (Verbeke et al., 2001), em que os $\gamma_{i}$ 's são considerados como efeitos perturbadores e é adequada quando estamos interessados em um subconjunto de coeficientes de regressão que não varia entre os indivíduos.

Um caso especial do modelo linear normal de efeitos mistos muito utilizado na prática ocorre quando temos

$$
\mathbf{D}=\sigma_{g}^{2} \mathbf{I}_{q} \quad \mathbf{R}_{i}=\sigma_{e}^{2} \mathbf{I}_{m_{i}},
$$

com $\sigma_{g}>0$ e $\sigma_{e}>0$, para $i=1, \ldots, n$. Nesse caso particular temos um modelo misto com 
interceptos aleatórios. A seguir descreveremos as duas abordagem para a inferência no modelo linear normal de efeitos mistos.

\subsubsection{Abordagem Marginal}

O modelo linear normal de efeitos mistos proposto por Laird e Ware (1982) satisfaz:

$$
\begin{aligned}
& \mathbf{Y}_{i}=\mathbf{X}_{i} \boldsymbol{\beta}+\mathbf{Z}_{i} \boldsymbol{\gamma}_{i}+\boldsymbol{\epsilon}_{i}, \\
& \boldsymbol{\epsilon}_{i} \stackrel{\text { ind }}{\sim} \mathrm{N}_{m_{i}}\left(\mathbf{0}, \mathbf{R}_{i}\right),, i=1, \ldots, n, \\
& \gamma_{i} \stackrel{\text { iid }}{\sim} \mathrm{N}_{q}(\mathbf{0}, \mathbf{D}), i=1, \ldots, n, \\
& \gamma_{1}, \ldots, \gamma_{n}, \boldsymbol{\epsilon}_{1}, \ldots, \boldsymbol{\epsilon}_{n}, \quad \text { são independentes. }
\end{aligned}
$$

em que $\mathbf{R}_{i}$ é uma matriz $m_{i} \times m_{i}$ de variância-covariância definida positiva que depende de $i$ somente através de sua dimensão $m_{i}$, $\mathbf{D}$ é uma matriz $q \times q$ de variância-covariância definida positiva. Segue de (2.4) que condicional aos efeitos aleatórios $\boldsymbol{\epsilon}_{i}, \mathbf{Y}_{i}$ segue uma distribuição normal com vetor de média $\mathbf{X}_{i} \boldsymbol{\beta}$ e matriz de variância-covariância $\boldsymbol{\Sigma}_{i}=\mathbf{Z}_{i} \mathbf{D} \mathbf{Z}_{i}^{\top}$.

Denotando a função densidade de probabilidade (fdp) de $\mathbf{Y}_{i} \mid \boldsymbol{\gamma}_{i}$ e a fdp de $\gamma_{i}$ por $f\left(\mathbf{y}_{i} \mid \boldsymbol{\gamma}_{i}\right)$ e $f\left(\gamma_{i}\right)$ respectivamente, temos que a fdp marginal de do vetor $\mathbf{Y}_{i}$ é dada por

$$
f\left(\mathbf{y}_{i}\right)=\int_{\mathbb{R}^{q}} f\left(\mathbf{y}_{i} \mid \boldsymbol{\gamma}_{i}\right) f\left(\boldsymbol{\gamma}_{i}\right) d \boldsymbol{\gamma}_{i}
$$

a qual pode ser mostrado que é a fdp de uma normal multivariada de dimensão $m_{i}$ com vetor de média $\mathbf{X}_{i} \boldsymbol{\beta}$ e matriz de variância-covariância $\mathbf{V}_{i}=\mathbf{Z}_{i} \mathbf{D} \mathbf{Z}_{i}^{\top}+\mathbf{R}_{i}$, isto é

$$
\mathbf{Y}_{i} \sim \mathrm{N}_{m_{i}}\left(\mathbf{X}_{i} \boldsymbol{\beta}, \mathbf{Z}_{i} \mathbf{D} \mathbf{Z}_{i}^{\top}+\mathbf{R}_{i}\right)
$$

A definição do modelo (2.3) através das fdp's $f\left(\mathbf{y}_{i} \mid \boldsymbol{\gamma}_{i}\right)$ e $f\left(\boldsymbol{\gamma}_{i}\right)$ é chamada a formulação hierárquica do modelo linear misto e a correspondente distribuição normal marginal de $\mathbf{Y}_{i}$ é chamada a formulação marginal do modelo.

Sobre abordagem marginal, a inferência estatística para o modelo linear normal de efeitos mistos é baseada no método da máxima verossimilhança ou no método da máxima verossimilhança restrita, (veja por exemplo Laird e Ware, 1982). Aqui, a média marginal $\mathrm{E}\left[\mathbf{Y}_{i}\right]=\mathbf{X}_{i} \boldsymbol{\beta}$, é a média sobre todos os efeitos aleatórios e a inferência estatística para cada individuo é feita pelo condicionamento sobre os efeitos aleatórios $\gamma_{i}$.

A estrutura de correlação entre as observações é indicada pela matriz $\mathbf{V}_{i}=\mathbf{Z}_{i} \mathbf{D} \mathbf{Z}_{i}^{\top}+\mathbf{R}_{i}$, essa estrutura pode ser induzida por meio do termo da variabilidade entre individuos $\mathbf{Z}_{i} \mathbf{D} \mathbf{Z}_{i}^{\top}$, ou por meio da matriz de variância-covariância das observações dentro do indivíduo, $\mathbf{R}_{i}$. Assim, mesmo que os erros $\boldsymbol{\epsilon}_{i}$ sejam independentes, as observações $\mathbf{Y}_{i}$ podem ser correlacionadas devido à variabilidade entre os indivíduos.

Diferentes estruturas de correlação para as matrizes $\mathbf{D}$ e $\mathbf{R}_{i}$ têm sido consideradas por varios autores, veja por ejemplo, Fitzmaurice et al. (2004); Pinheiro e Bates (2000); Rocha (2004); Weiss (2005), enquanto que mais detalhes sobre os métodos de estimação de máxima verossimilhança e máxima verossimilhança restrita podem ser encontrados em Laird e Ware (1982) e Lindstrom e Bates (1988).

\subsubsection{Abordagem Condicional}

A abordagem condicional é uma alternativa à estimação clássica pelo método da máxima verossimilhança e consiste na estimação dos parâmetros por meio da maximização da função de 
verossimilhança dos dados $\mathbf{Y}_{i}$, condicional a estatísticas suficientes para os parâmetros perturbadores $\gamma_{i}$.

A principal vantagem da inferência condicional é que não são necessárias suposições adicionais em relação aos parâmetros de perturbação $\gamma_{i}$ (Molenberghs e Verbeke, 2005; Verbeke et al., 2001), e a inferência torna-se disponível para os parâmetros de interesse desconsiderando completamente os parâmetros de perturbação sem perda de qualquer informação; porém uma desvantagem é que precisam ser encontradas estatísticas suficientes para os $\gamma_{i}$ e, posteriormente calcular e maximizar a função de verossimilhança condicional dos dados condicional a essas estatísticas. Detalhes desta abordagem podem ser consultados em Verbeke e Molenberghs (2009).

\subsection{Distribuições Normal-Assimétrica e $t$-Assimétrica}

Nesta seção vamos apresentar a classe de distribuições normal-assimétrica e $t$-assimétrica introduzidas por Azzalini (1985) e Azzalini e Capitanio (2003), respectivamente; as quais são extensões dos modelos normal e $t$ de Student, respectivamente, e permitem modelar dados com maior grau de assimetria e curtose do que essas distribuições.

\subsubsection{Distribuição Normal-Assimétrica}

A família de densidades normal-assimétrica foi introduzida inicialmente por Azzalini (1985) como uma alternativa razoável ao modelo normal clássico, sendo muito útil na prática pela robustez do modelo contra afastamentos da suposição de normalidade dos dados sobre estudo.

Definição 1 Dizemos que uma variável aleatória $X$ tem uma distribuição normal-assimétrica com parâmetro de locação $\xi$, parâmetro de escala $\eta>0$ e parâmetro de assimetria $\lambda$, se sua fdp é da forma

$$
f_{S N}(x \mid \xi, \eta, \lambda)=\frac{2}{\eta} \phi\left(\frac{x-\xi}{\eta}\right) \Phi\left(\lambda\left(\frac{x-\xi}{\eta}\right)\right), \quad x \in \mathbb{R} .
$$

Cometendo um pequeno abuso de notação, escreveremos $X \sim \operatorname{SN}(\xi, \eta, \lambda)$ (Skew-Normal). Quando $\xi=0$ e $\eta=1$ temos a distribuição $\mathrm{SN}$ padronizada e escreveremos $\mathrm{SN}(0,1, \lambda) \equiv \operatorname{SN}(\lambda)$. A densidade SN é simétrica quando $\lambda=0$ e em tal caso a densidade normal é obtida. Quando $\lambda$ tende a $+\infty$ (tende a $-\infty$ ), a variável aleatória $X$ tende a uma variável aleatória mitade-normal (half-normal) positiva (negativa) (Azzalini, 1985). Exemplos da densidade (2.6) para diferentes valores de $\lambda$ são mostrados na Figura 2.1.

Seja $Z \sim \operatorname{SN}(\lambda)$ então a média e a variância de $Z$ são dados respectivamente por

$$
\mathrm{E}[Z]=\sqrt{\frac{2}{\pi}} \frac{\lambda}{\sqrt{1+\lambda^{2}}}, \quad \operatorname{Var}[Z]=1-\frac{2 \lambda^{2}}{\pi\left(1+\lambda^{2}\right)}
$$

O principal problema da densidade SN é que sob a hipótese de simetria dos dados, isto é $\lambda=0$, a matriz de informação de Fisher é singular e portanto as condições de regularidade não são satisfeitas para a inferência vía máxima verossimilhança, limitando assim o uso do estimador de máxima verossimilhança na construção de testes de hipóteses baseados na estatística de razão de verossimilhanças.

Para resolver a dificuldade de singularidade da matriz de informação, Arellano-Valle e Azzalini (2008) propuseram a parametrização centrada da distribuição SN quando $\lambda=0$, definido a variável aleatória $X=\xi+\eta\left(\frac{Z-\mathrm{E}[Z]}{\sqrt{\operatorname{Var}[Z]}}\right)$, em que $\xi$ e $\eta$ são parâmetros da variável aleatória $X$ e $Z \sim \operatorname{SN}(\lambda)$. Neste trabalho iremos considerar a forma usual da distribuição SN dada em (2.6), 


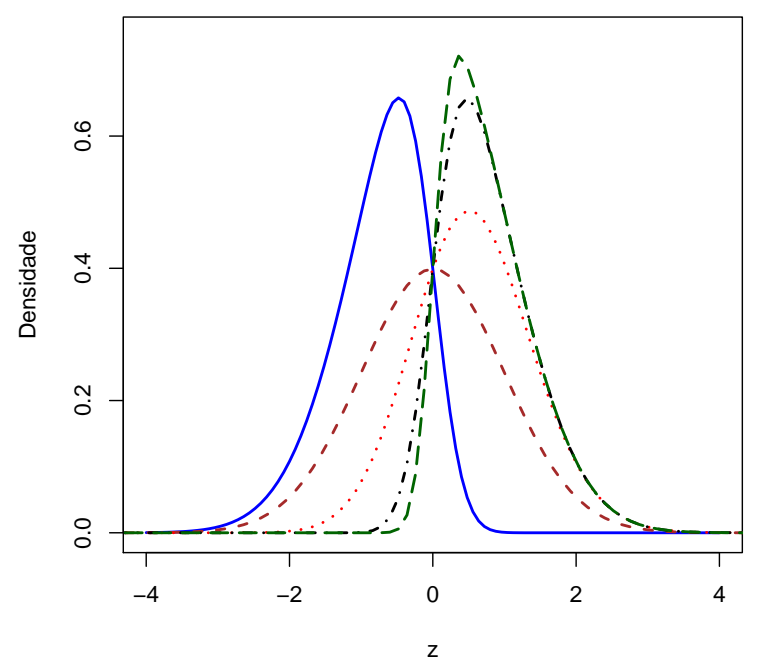

Figura 2.1: Função densidade de probabilidade da $\mathrm{SN}(\lambda)$ para $\lambda=-3$ (linha sólida), $\lambda=0$ (linha tracejada), $\lambda=1$ (linha pontilhada), $\lambda=3$ (linha pontilhada-tracejada) e $\lambda=5$ (linha longa-tracejada).

enquanto que mais detalhes sob a parametrização centrada da distribuição SN podem ser vistos em Arellano-Valle e Azzalini (2008).

\subsubsection{Distribuição Normal-Assimétrica Truncada}

A distribuição normal-assimétrica truncada a qual denotaremos por TSN (Truncated SkewNormal) foi introduzida e estudada em detalhe por Flecher et al. (2010). Definimos a distribuição TSN a partir da densidade SN dada em (2.6) como segue

Definição 2 Seja $X \sim S N(\xi, \eta, \lambda)$ e $a<b$, tal que $P(a<X<b)>0$. A variável aleatória $Y$ tem uma distribuição normal-assimétrica truncada no intervalo $(a, b)$, se esta tem a mesma distribuição que $X \mid X \in(a, b)$. Neste caso, escrevemos $Y \sim T S N_{(a, b)}(\xi, \eta, \lambda)$.

Como uma consequência obvia da Definição 2, podemos obter a densidade de $Y \sim \operatorname{TSN}_{(a, b)}(\xi, \eta, \lambda)$ dada por

$$
\begin{aligned}
f_{T S N}(y \mid \xi, \eta, \lambda ;(a, b))=\frac{2}{\eta} & \phi\left(\frac{y-\xi}{\eta}\right) \Phi\left(\lambda\left(\frac{y-\xi}{\eta}\right)\right) \\
& \times\left[F_{S N}\left(\frac{b-\xi}{\eta}\right)-F_{S N}\left(\frac{a-\xi}{\eta}\right)\right]^{-1}
\end{aligned}
$$

e $f_{T S N}(y \mid \xi, \eta, \lambda ;(a, b))=0$ em outro caso, em que $F_{S N}(\cdot)$ é a fda da distribuição normalassimetrica padronizada. 
Proposição 1 Seja $Y \sim T S N_{(a, b)}(\xi, \eta, \alpha)$. Então, para $a<b$ a $\mathrm{E}\left[Y^{r}\right]$ para $r=1,2$ é dada por

$$
\begin{aligned}
\mathrm{E}[Y] & =\sqrt{\frac{2}{\pi}} \frac{\lambda}{\sqrt{1+\lambda^{2}}} \frac{\Phi\left(\lambda_{*} z_{b}\right)-\Phi\left(\lambda_{*} z_{a}\right)}{F_{S N}\left(z_{b}\right)-F_{S N}\left(z_{a}\right)}-\frac{f_{S N}\left(z_{b}\right)-f_{S N}\left(z_{a}\right)}{F_{S N}\left(z_{b}\right)-F_{S N}\left(z_{a}\right)} \\
\mathrm{E}\left[Y^{2}\right] & =1-\sqrt{\frac{2}{\pi}} \frac{\lambda}{1+\lambda^{2}} \frac{\phi\left(\lambda_{*} z_{b}\right)-\phi\left(\lambda_{*} z_{a}\right)}{F_{S N}\left(z_{b}\right)-F_{S N}\left(z_{a}\right)}-\frac{z_{b} f_{S N}\left(z_{b}\right)-z_{a} f_{S N}\left(z_{a}\right)}{F_{S N}\left(z_{b}\right)-F_{S N}\left(z_{a}\right)}
\end{aligned}
$$

em que $\phi(\cdot)$ e $\Phi(\cdot)$ são a fdp e a fda da distribuição normal padronizada, respectivamente; $f_{S N}(\cdot)$ e $F_{S N}(\cdot)$ são a fdp e a fda da distribuição $S N$ padronizada respectivamente, $z_{a}=\frac{a-\xi}{\eta}, z_{b}=\frac{b-\xi}{\eta}$ $e \lambda_{*}=\sqrt{1+\lambda^{2}}$.

A Proposição 1 é um resultado que pode ser obtido usando as expressões recorrentes dadas em Flecher et al. (2010).

\subsubsection{Distribuição $t$-Assimétrica}

Branco e Dey (2001) introduziram a distribuição $t$-assimétrica como um caso particular de uma classe mais geral de distribuições chamada de distribuições elípticas-assimétricas multivardas, enquanto que Azzalini e Capitanio (2003) discutiram a distribuição t-assimétrica usando um procedimento geral que perturba a densidade da distribuição $t$ de Student e gera um conjunto de densidades assimétricas. Neste trabalho iremos usar a distribuição $t$-assimétrica introduzida por Azzalini e Capitanio (2003) definida a seguir.

Definição 3 Dizemos que uma variável aleatória $X$ tem uma distribuição t-assimétrica com parâmetro de locação $\xi$, parâmetro de escala $\eta>0$, parâmetro de assimetria $\lambda$ e $\nu>0$ graus de liberdade, se sua fdp é da forma

$$
f_{S T}(x \mid \xi, \eta, \lambda, \nu)=\frac{2}{\eta} t\left(\frac{x-\xi}{\eta} \mid \nu\right) T\left(\lambda\left(\frac{x-\xi}{\eta}\right) \sqrt{\frac{\nu+1}{Q_{x}+\nu}} \mid \nu+1\right), \quad x \in \mathbb{R}
$$

em que $Q_{x}=((x-\xi) / \eta)^{2}, t(\cdot \mid \nu)$ e $T(\cdot \mid \nu)$ são a fdp e a fda dadistribuição $t$ de Student padronizada dadas em (2.1) e (2.2), respectivamente.

Escreveremos $X \sim \operatorname{ST}(\xi, \eta, \lambda, \nu)($ Skew- $T)$. Quando $\xi=0$ e $\eta=1$ temos a distribuição ST padronizada e escreveremos $\mathrm{ST}(0,1, \lambda, \nu) \equiv \mathrm{ST}(\lambda, \nu)$.

\subsection{Distribuições Potência-Alfa}

A grande maioria do desenvolvimento deste trabalho é baseada nas distribuições da família potência-alfa as quais têm mostrado ser alternativas viáveis para modelar dados assimétricos (veja por exemplo Gupta e Gupta, 2008; Pewsey et al., 2012), em particular as distribuições normal-potência (Gupta e Gupta, 2008) e $t$-potência (Zhao e Kim, 2016). A seguir apresentamos as definições e propriedades básicas dessas distribuições.

Começaremos definindo a classe de distribuições potência-alfa e potência-alfa truncada, logo em seguida introduzimos as distribuições potência-normal e potência-t.

Definição 4 Dizemos que a variável aleatória $X$ tem uma distribuição univariada potência-alfa com parâmetro de locação $\xi$, parâmetro de escala $\eta>0$ e parâmetro de forma $\alpha>0$, se a fdp de 
$X$ é dada por

$$
f_{A P}(x \mid \xi, \eta, \alpha)=\frac{\alpha}{\eta} f\left(\frac{x-\xi}{\eta}\right)\left\{F\left(\frac{x-\xi}{\eta}\right)\right\}^{\alpha-1}, \quad x \in \mathbb{R}
$$

em que $F(\cdot)$ é uma função de distribuição absolutamente contínua com fdp dada por $f=d F$.

Escreveremos $X \sim \operatorname{AP}(\xi, \eta, \alpha)$ (Alpha-Power). Quando $\xi=0$ e $\eta=1$ temos a distribuição potência-alfa padronizada e escrevemos $X \sim \operatorname{AP}(\alpha)$.

O modelo AP foi derivado num contexto hidrológico como uma estatística de ordem fracionária por Durrans (1992), mas este modelo tem sido discutido amplamente por outros autores (Veja Arnold, 2004; Gupta e Gupta, 2008). O modelo AP constitui uma alternativa à família de distribuições que podem ajustar alto grau de assimetria e/ou curtose em um conjunto de dados, por exemplo a família de distribuições normal-assimétrica de Azzalini (1985).

Se $Z \sim \operatorname{AP}(\alpha)$, os momentos da variável $Z$ não têm uma forma fechada mas fazendo a mudança de variável $U=F(Z)$, o $r$-ésimo momento da variável $Z$ pode ser escrito como

$$
\mathrm{E}\left[Z^{r}\right]=\alpha \int_{0}^{1}\left[F^{-1}(u)\right]^{r} u^{\alpha-1} d u
$$

em que $F^{-1}(\cdot)$ é a inversa da função $F(\cdot)$. Esta esperança concorda com a esperança da expressão $\left[F^{-1}(U)\right]^{r}$ de uma variável aleatória $U$ com distribuição beta de parâmetros $\alpha$ e 1 , isto é,

$$
\mathrm{E}\left[Z^{r}\right]=\mathrm{E}\left[\left(F^{-1}(U)\right)^{r}\right]
$$

em que $U \sim \operatorname{Beta}(\alpha, 1)$.

Definição 5 Seja $X \sim A P(\xi, \eta, \alpha)$ e $a<b$, tal que $P(a<X<b)>0$. A variável aleatória $Y$ tem uma distribuição AP truncada no intervalo $(a, b)$, se esta tem a mesma distribuição que $X \mid X \in(a, b)$. Neste caso, escrevemos $Y \sim T A P_{(a, b)}(\xi, \eta, \alpha)$.

Como uma consequência obvia da Definição 5, podemos obter a densidade de $Y \sim T A P$ dada por

$$
f_{T A P}(y \mid \xi, \eta, \alpha ;(a, b))=f_{A P}(y \mid \xi, \eta, \alpha)\left[F_{A P}\left(\frac{b-\xi}{\eta}\right)-F_{A P}\left(\frac{a-\xi}{\eta}\right)\right]^{-1}
$$

e $f_{T A P}(y \mid \xi, \eta, \alpha ;(a, b))=0$ em outro caso, em que $F_{A P}(\cdot)$ é a fda da distribuição AP padronizada.

\subsubsection{Distribuição Normal-Potência}

Durrans (1992) considera o modelo (2.10) para o caso em que $F(\cdot)=\Phi(\cdot)$ é fda da distribuição normal padrão, e neste caso a família obtida é chamada de classe de distribuições normal-potência assimétrica e é denotada por PN (power-normal). A fdp de $X$ é dada por

$$
f_{P N}(x \mid \xi, \eta, \alpha)=\frac{\alpha}{\eta} \phi\left(\frac{x-\xi}{\eta}\right)\left\{\Phi\left(\frac{x-\xi}{\eta}\right)\right\}^{\alpha-1}, \quad x \in \mathbb{R}, \alpha \in \mathbb{R}^{+}
$$

Escreveremos $X \sim \operatorname{PN}(\xi, \eta, \alpha)$ e se segue portanto que $\mathrm{PN}(0,1, \alpha) \equiv \mathrm{PN}(\alpha)$ denota o modelo PN padronizado.

Quando $\alpha=1$, o modelo (2.13) se reduz à distribuição normal de média $\xi$ e variância $\eta^{2}$; e para $\alpha=2$, o modelo contém a fdp da distribuição normal-assimétrica de Azzalini (1985) 
para $\lambda=1$. Valores de $\alpha$ tais que $0<\alpha<1(\alpha>1)$ correspondem a uma assimetria negativa (positiva) na densidade (2.13). Exemplos da densidade PN são mostrados na Figura 2.2.

As distribuição PN e as suas principais propriedades têm sido estudadas amplamente por Durrans (1992); Gupta e Gupta (2004, 2008); Lehmann (1953) e Pewsey et al. (2012). Embora o modelo PN tem intervalo de assimetria mais curto do que o modelo SN de Azzalini (1985), o modelo PN pode ser capaz de ajustar dados com coeficiente de curtose maior do que o modelo SN e além disso, apresenta matriz de informação não singular, (veja Pewsey et al., 2012). Finalmente extensões ao caso multivariado desse modelo são discutidas por Martínez-Flórez et al. (2013a) e Kundu e Gupta (2013).

\subsubsection{Distribuição $t$-Potência}

Zhao e Kim (2016) estudaram o caso para $F(\cdot)=T(\cdot)$ no modelo $(2.10)$, em que $T(\cdot)$ é a fda da distribuição $t$ de Student. Neste caso a fdp da variável aleatória $X$ é dada por

$$
f_{P T}(x \mid \xi, \eta, \alpha, \nu)=\frac{\alpha}{\eta} t\left(\frac{x-\xi}{\eta} \mid \nu\right)\left\{T\left(\frac{x-\xi}{\eta} \mid \nu\right)\right\}^{\alpha-1}, \quad x \in \mathbb{R}, \alpha \in \mathbb{R}^{+}
$$

em que $\nu>0$ são os graus de liberdade.

A classe de distribuições em (2.14) é chamada de classe de distribuições t-potência assimétrica, e escreveremos $X \sim \mathrm{PT}(\xi, \eta, \alpha, \nu)$. Se segue que $\mathrm{PT}(0,1, \alpha, \nu) \equiv \mathrm{PT}(\alpha, \nu)$ denota o modelo PT padronizado. Quando $\alpha=1$, o modelo contém a distribuição $t$ de Student e para $\alpha=2$ contém a fdp da distribuição $t$ assimétrica com $\lambda=1$ estudada en detalhe por Azzalini e Capitanio (2003). Exemplos da densidade (2.14) são mostrados na Figura 2.2.

$\mathrm{O}$ modelo $t$-potência generaliza o amplamente conhecido modelo $t$ de Student e para um mesmo grau de liberdade apresenta maior amplitude de assimetria e curtose em relação ao modelo $t$-assimétrico de Azzalini e Capitanio (2003), assim o modelo $t$-potência é capaz de lidar com dados com caudas ainda mais pesadas do que o modelo normal-assimétrico (veja Zhao e Kim, 2016).

Bem como no modelo normal-potência, o modelo t-potência apresenta matrix de informação não-singular e portanto podem ser usadas estatísticas de razão de verossimilhanças para a construção de testes de hipótese a partir de grandes amostras. Mais detalhes deste modelo podem ser consultadas em Zhao e Kim (2016).

\subsubsection{Momentos nos Modelos Normal-Potência e $t$-Potência}

Os momentos de uma variável aleatória com distribuição PN e PT podem ser obtidos a partir de (2.11) considerando as respectivas fdp's das distribuições PN e PT. Assim se $X \sim \operatorname{PN}(\xi, \eta, \alpha)$ ou $X \sim \operatorname{PT}(\xi, \eta, \alpha, \nu)$, segue que

$$
\mathrm{E}\left[X^{r}\right]=\sum_{k=0}^{r}\left(\begin{array}{l}
r \\
k
\end{array}\right) \xi^{k} \eta^{r-k} \mu_{r-k}
$$

em que $\mu_{j}$ é o $j$-ésimo momento de uma variável aleatória $Y \sim \operatorname{PN}(\alpha)$ ou $Y \sim \operatorname{PT}(\alpha, \nu)$ padronizada, respectivamente, isto é,

$$
\mu_{j}= \begin{cases}\int_{0}^{1} \alpha\left[\Phi^{-1}(u)\right]^{j} u^{\alpha-1} d u, & \text { se } Y \sim \operatorname{PN}(\alpha) \\ \int_{0}^{1} \alpha\left[T^{-1}(u \mid \nu)\right]^{j} u^{\alpha-1} d u, & \text { se } Y \sim \operatorname{PT}(\alpha, \nu)\end{cases}
$$




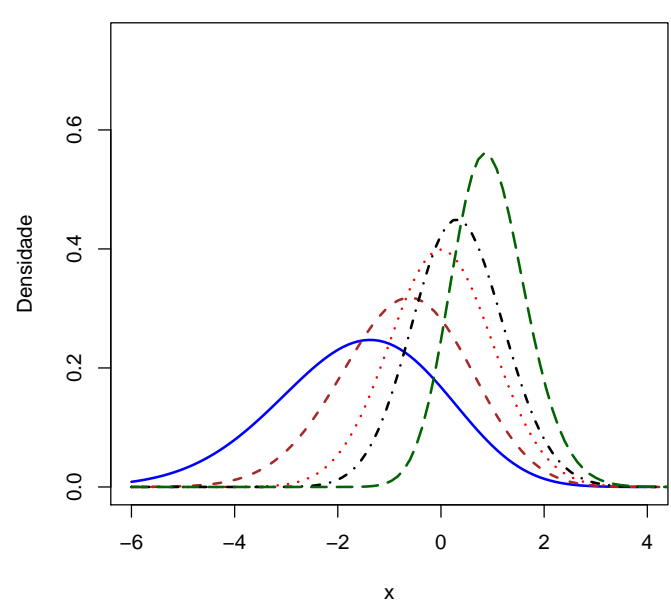

(a)

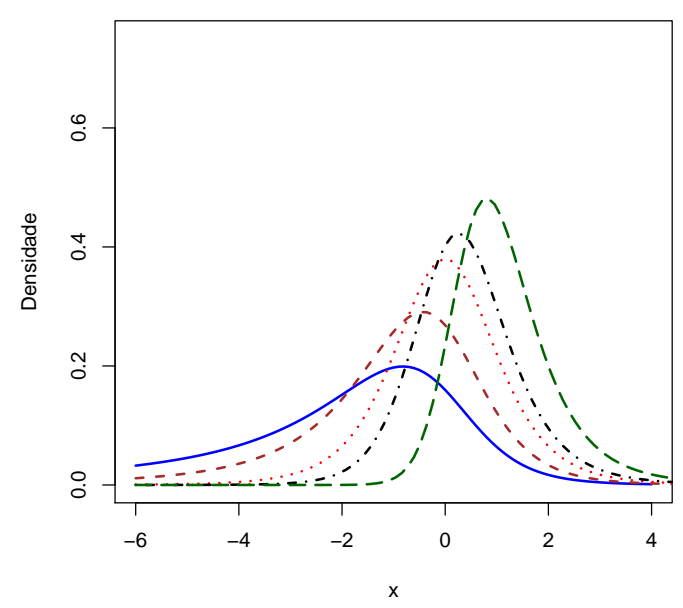

(b)

Figura 2.2: Função densidade de probabilidade de: (a) Distribuição $\mathrm{PN}(\alpha)$ para $\alpha=0.25$ (linha sólida), $\alpha=0.50$ (linha tracejada), $\alpha=1.0$ (linha pontilhada), $\alpha=1.5$ (linha pontilhada-tracejada) e $\alpha=3.5$ (linha longa-tracejada). (b) Distribuição $\mathrm{PT}(\alpha, \nu=5$ ) para $\alpha=0.25$ (linha sólida), $\alpha=0.50$ (linha tracejada), $\alpha=1.0$ (linha pontilhada), $\alpha=1.5$ (linha pontilhada-tracejada) e $\alpha=3.5$ (linha longatracejada)

para $j=1,2, \ldots$ e $\mu_{0}=1$.

Da Definição 5 se segue que se $Y \sim \operatorname{PN}(\xi, \eta, \alpha)$ então a pdf da variável aleatória normalpotência truncada, denotada TPN (Truncated Power-Normal) no intervalo $(a, b)$, é dada por

$$
\begin{aligned}
f_{T P N}(y \mid \xi, \eta, \alpha ;(a, b))=\frac{\alpha}{\eta} & \phi\left(\frac{y-\xi}{\eta}\right)\left\{\Phi\left(\frac{y-\xi}{\eta}\right)\right\}^{\alpha-1} \\
\times & {\left[F_{P N}\left(\frac{b-\xi}{\eta}\right)-F_{P N}\left(\frac{a-\xi}{\eta}\right)\right]^{-1} }
\end{aligned}
$$

em que $F_{P N}(\cdot)$ é a fda da distribuição normal-potência padronizada.

Segue também da Definição 5 que $Y \sim \operatorname{PT}(\xi, \eta, \alpha, \nu)$, a fdp de uma variável aleatória $t$ potência truncada, denotada TPT (Truncated Power-t) no intervalo $(a, b)$ é dada por

$$
\begin{aligned}
& f_{T P T}(y \mid \xi, \eta, \alpha, \nu ;(a, b))=\frac{\alpha}{\eta} t\left(\frac{y-\xi}{\eta} \mid \nu\right)\left\{T\left(\frac{y-\xi}{\eta} \mid \nu\right)\right\}^{\alpha-1} \\
& \times\left[F_{P T}\left(\frac{b-\xi}{\eta}\right)-F_{P T}\left(\frac{a-\xi}{\eta}\right)\right]^{-1}
\end{aligned}
$$

em que $F_{P T}(\cdot)$ é a fda da distribuição $t$-potência padronizada.

Proposição 2 Se $Y \sim T P N_{(a, b)}(\xi, \eta, \alpha)$, então o r-ésimo momento da variável aleatória $Y$ é dado por

$$
\mathrm{E}\left[Y^{r}\right]=\sum_{k=0}^{r} \xi^{k} \eta^{r-k} \mu_{r-k}
$$


em que

$$
\mu_{s}=\left[F_{P N}\left(z_{b}\right)-F_{P N}\left(z_{a}\right)\right]^{-1} \int_{\Phi\left(z_{a}\right)}^{\Phi\left(z_{b}\right)} \alpha\left[\Phi^{-1}(u)\right]^{s} u^{\alpha-1} d u, \quad s=1,2, \ldots
$$

$e \mu_{0}=1, \operatorname{com} F_{P N}(z)=[\Phi(z)]^{\alpha}$ e $z_{c}=\frac{c-\xi}{\eta}$.

Proposição 3 Seja $Z \sim P T(\alpha, \nu)$, então segue que $E\left[Z^{r} \mid Z+c>0\right]=m_{r}(c \mid \nu)$, com

$$
m_{r}(c \mid \nu)=\frac{1}{1-\{T(-c \mid \nu)\}^{\alpha}} \int_{T(-c \mid \nu)}^{1}\left[T^{-1}(u \mid \nu)\right]^{r} \alpha u^{\alpha-1} d u .
$$

em que $T^{-1}(\cdot \mid \nu)$ é a função inversa da fda $T(\cdot \mid \nu)$.

Proposição 4 Seja $Z \sim P T(\alpha, \nu)$, e defina $r(Z \mid \nu)=t(Z \mid \nu) / T(Z \mid \nu)$. Então

(i) $E\left\{[r(Z \mid \nu)]^{k}\left(1+\frac{Z^{2}}{\nu}\right)^{-m / 2} Z^{n} \mid Z+c>0\right\}=\frac{[t(0 \mid \nu)]^{k} a_{k m n}(-c \mid \nu)}{1-\{T(-c \mid \nu)\}^{\alpha}}$, em que

$$
a_{k m n}(-c \mid \nu)=\int_{T(-c \mid \nu)}^{1}\left[T^{-1}(u \mid \nu)\right]^{n}\left\{1+\frac{\left[T^{-1}(u \mid \nu)\right]^{2}}{\nu}\right\}^{-\frac{k(\nu+1)+m}{2}} \alpha u^{\alpha-k-1} d u
$$

(ii) $E\left\{[r(Z \mid \nu)]^{k} \log \left(1+\frac{Z^{2}}{\nu}\right) Z^{n} \mid Z+c>0\right\}=\frac{[t(0 \mid \nu)]^{k} a_{k n}(-c \mid \nu)}{1-\{T(-c \mid \nu)\}^{\alpha}}$, em que

$$
a_{k n}(-c \mid \nu)=\int_{T(-c \mid \nu)}^{1} \log \left\{1+\frac{\left[T^{-1}(u \mid \nu)\right]^{2}}{\nu}\right\}\left[T^{-1}(u \mid \nu)\right]^{n} \alpha u^{\alpha-k-1} d u
$$

(iii) $E\left\{[r(Z \mid \nu)]^{k}\left[b_{01}(Z \mid \nu)\right]^{m} Z^{n} \mid z+c>0\right\}=\frac{[t(0 \mid \nu)]^{k} b_{k m n}(-c \mid \nu)}{1-\{T(-c \mid \nu)\}^{\alpha}}$, em que

$$
b_{k m n}(-c \mid \nu)=\int_{T(-c \mid \nu)}^{1}\left[b_{01}\left(T^{-1}(u \mid \nu)\right)\right]^{m}\left[T^{-1}(u \mid \nu)\right]^{n} \alpha u^{\alpha-k-1} d u
$$

com $b_{01}(c \mid \nu)=\int_{-\infty}^{c}\left\{\log \left(1+\frac{s^{2}}{\nu}\right)\right\} \frac{t(s \mid \nu)}{T(c \mid \nu)} d s$.

\subsubsection{Distribuição Normal-Potência Multivariada}

Extensões do modelo normal-potência univariado (2.13) ao caso multivariado têm sido consideradas por alguns autores (Kundu e Gupta, 2013; Martínez-Flórez et al., 2013a). Inicialmente, a distribuição normal-potência bivariada foi introduzida e estudada por Kundu e Gupta (2013). Os autores obtiveram essa distribuição usando a cópula de Clayton e considerando que as distribuições marginais seguem distribuições normal-potência. Os autores também propuseram a extensão do modelo normal-potência para $p$ variáveis.

Por outro lado, Martínez-Flórez et al. (2013a) propuseram a extensão multivariada de modelo potência-alfa, seguindo a teoria de distribuições multivariadas a partir de distribuições condicionais proposto por Arnold et al. (1999). 
A seguir consideramos o modelo normal-potência multivariado proposto por Kundu e Gupta (2013) que será usado para o desenvolvimento do modelo linear misto potência-normal no Capítulo 4 .

Definição 6 O vetor aleatório $\mathbf{Y}=\left(Y_{1}, \ldots, Y_{p}\right)^{\top}$ tem uma distribuição normal-potência $p$ variada com vetor de parâmetros $\boldsymbol{\alpha}=\left(\alpha_{1}, \ldots, \alpha_{p}, \alpha\right)^{\top}$; a qual é denotada por $P N_{p}(\boldsymbol{\alpha})$, se $\mathbf{Y}$ tem fdp conjunta dada por

$$
f(\mathbf{y} ; \boldsymbol{\alpha})=\frac{c_{1} \prod_{i=1}^{p} \phi\left(y_{i}\right)\left[\Phi\left(y_{i}\right)\right]^{-\alpha_{i} / \alpha-1}}{\left[\sum_{i=1}^{p}\left[\Phi\left(y_{i}\right)\right]^{-\alpha_{i} / \alpha}-(p-1)\right]^{\alpha+p}}, \quad y_{1}, \ldots, y_{p} \in \mathbb{R},
$$

em que $\alpha_{1}, \ldots, \alpha_{p}>0$, e $c_{1}=\Gamma(\alpha+p) / \alpha^{p} \Gamma(\alpha) \prod_{i=1}^{p} \alpha_{i}$.

A Figura 2.3 mostra alguns contornos da densidade normal-potência bivariada (normalpotência 2-variada, $P N_{2}$ ), a qual é obtida tomando $p=2 \mathrm{em}(2.19)$, para diferentes valores de $\boldsymbol{\alpha}=\left(\alpha_{1}, \alpha_{2}, \alpha\right)^{\top}$. Nota-se que esses contornos não são elípticos e podem ser assimétricos.

Como as distribuições marginais da fdp conjunta (2.19) seguem distribuições normal-potência univariada (veja, Kundu e Gupta, 2013), podemos usar a equação (2.15) para obter o vetor de médias e a matriz de variâncias-covariâncias do vetor aleatório $\mathbf{Y}$. Assim, se $\mathbf{Y} \sim \operatorname{PN}_{p}(\boldsymbol{\alpha})$, então o vetor de médias e a matriz de covariâncias de $\mathbf{Y}$ são dados por

$$
\mathrm{E}[\mathbf{Y}]=\left(\begin{array}{c}
\mu_{1} \\
\vdots \\
\mu_{p}
\end{array}\right)=\boldsymbol{\mu}, \quad \text { e } \quad \operatorname{Var}[\mathbf{Y}]=\left(\begin{array}{ccc}
\sigma_{11} & \cdots & \sigma_{1 p} \\
\vdots & \ddots & \vdots \\
\sigma_{p 1} & \cdots & \sigma_{p p}
\end{array}\right)=\boldsymbol{\Sigma},
$$

respectivamente, em que

$$
\mu_{i}=\int_{0}^{1} \alpha_{i} \Phi^{-1}(u) u^{\alpha_{i}-1} d u
$$

e

$$
\sigma_{i i}=\int_{0}^{1} \alpha_{i}\left[\Phi^{-1}(u)\right]^{2} u^{\alpha_{i}-1} d u-\left(\int_{0}^{1} \alpha_{i} \Phi^{-1}(u) u^{\alpha_{i}-1} d u\right)^{2}
$$

para $i=1, \ldots, p ;$ e

$$
\sigma_{i j}=\alpha(\alpha+1)\left\{\int_{1}^{+\infty} \int_{1}^{+\infty} \frac{\Phi^{-1}\left(u^{-\alpha / \alpha_{i}}\right) \Phi^{-1}\left(v^{-\alpha / \alpha_{j}}\right)}{(u+v-1)^{\alpha+2}} d u d v\right\}-\mu_{i} \mu_{j} .
$$

para $i \neq j$; com $i, j=1, \ldots, p$.

Seja $\mathbf{Y} \sim \operatorname{PN}_{p}(\boldsymbol{\alpha})$, então a versão de locação-escala do modelo (2.19) é obtida pela definição de $\mathbf{Z}=\boldsymbol{\xi}+\boldsymbol{\eta}^{\frac{1}{2}} \mathbf{Y}$, em que $\boldsymbol{\xi}=\left(\xi_{1}, \ldots, \xi_{p}\right)^{\top}$ e $\boldsymbol{\eta}=\operatorname{diag}\left(\eta_{1}^{2} \ldots, \eta_{p}^{2}\right)$, com $\xi_{1}, \ldots, \xi_{p} \in \mathbb{R}$, e $\eta_{1}, \ldots, \eta_{p} \in \mathbb{R}^{+}$. A fdp conjunta do vetor aleatório $\mathbf{Z}=\left(Z_{1}, \ldots, Z_{p}\right)^{\top}$ é dada por

$$
f(\mathbf{z} ; \boldsymbol{\xi}, \boldsymbol{\eta}, \boldsymbol{\alpha})=\frac{c_{2} \prod_{i=1}^{p} \phi\left(\frac{z_{i}-\xi_{i}}{\eta_{i}}\right)\left[\Phi\left(\frac{z_{i}-\xi_{i}}{\eta_{i}}\right)\right]^{-\alpha_{i} / \alpha-1}}{\left\{\sum_{i=1}^{p}\left[\Phi\left(\frac{z_{i}-\xi_{i}}{\eta_{i}}\right)\right]^{-\alpha_{i} / \alpha}-(p-1)\right\}^{\alpha+p}}
$$

em que $c_{2}=\Gamma(\alpha+p) / \alpha^{p} \Gamma(\alpha) \prod_{i=1}^{p} \alpha_{i} / \eta_{i}$, para $z_{1}, \ldots, z_{p} \in \mathbb{R}$. Além disso, o vetor de média e a matriz de covariâncias são dados por

$$
\mathrm{E}[\mathbf{Z}]=\boldsymbol{\xi}+\boldsymbol{\eta}^{\frac{1}{2}} \boldsymbol{\mu} \quad \text { e } \quad \operatorname{Var}[\mathbf{Z}]=\boldsymbol{\eta}^{\frac{1}{2}} \boldsymbol{\Sigma} \boldsymbol{\eta}^{\frac{1}{2}}
$$




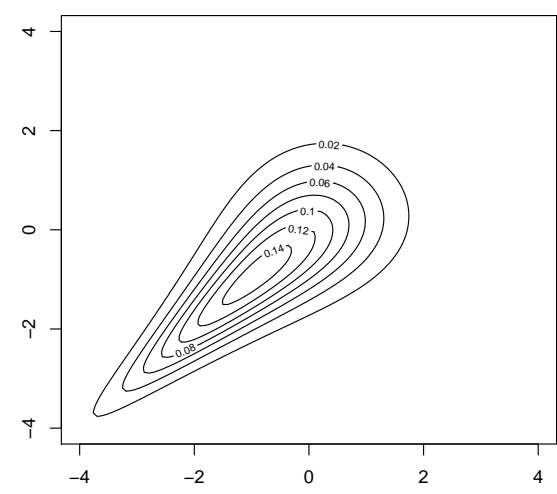

(a)

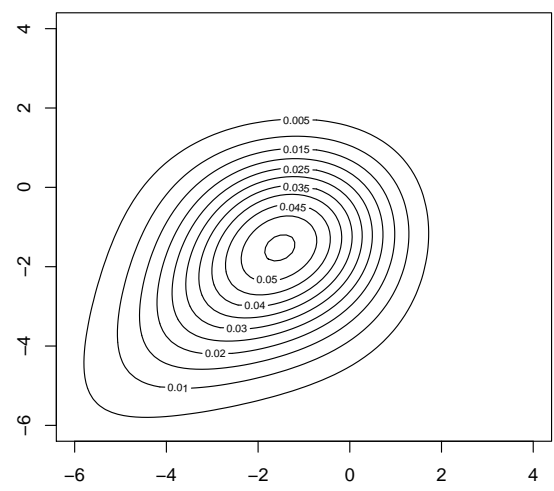

(c)

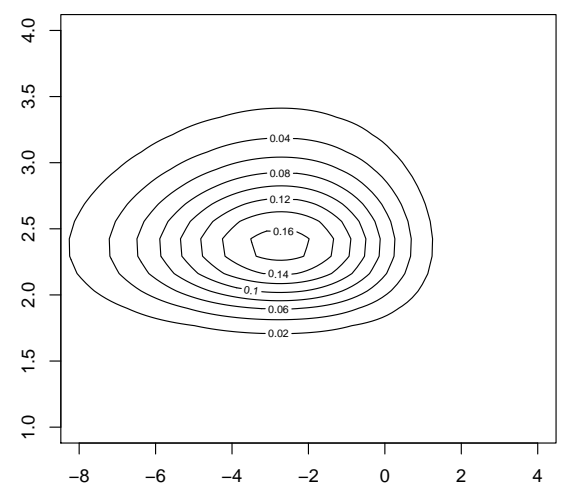

(b)

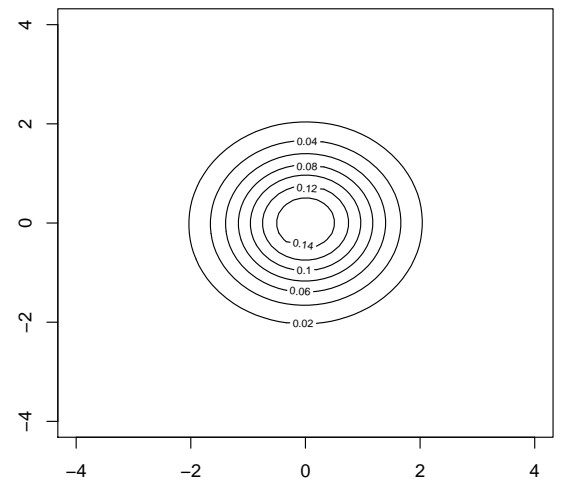

(d)

Figura 2.3: Contornos da distribuição normal-potência 2-variada para of $\boldsymbol{\alpha}=\left(\alpha_{1}, \alpha_{2}, \alpha\right)^{\top}$ : (a) $(0.5,0.5$, $0.5)$, (b) $(0.10,100,100)$, (c) $(0.20,0.20,2.0)$ e (d) $(1.0,1.0,100)$.

com $\boldsymbol{\mu}$ e $\boldsymbol{\eta}$ dados como em (2.20). Esta extensão é denotada por $\mathbf{Z} \sim \mathrm{PN}_{p}(\boldsymbol{\xi}, \boldsymbol{\eta}, \boldsymbol{\alpha})$.

Propriedades adicionais da distribuição potência-normal $p$-variada podem ser econtradas em Kundu e Gupta (2013), especialmente para o caso $p=2$.

\subsection{Quadratura de Gauss-Hermite}

O método da quadratura de Gauss é usado para aproximar integrais de funções em relação a um kernel dado por uma média ponderada do integrando avaliado em abscisas predeterminadas.

Neste trabalho usaremos o método da quadratura de Gauss-Hermite para aproximar as integrais envolvidas nas funções de verossimilhanças dos modelos mistos estudados nos Capítulos seguintes, portanto é necessário descrever brevemente esse método. 


\subsubsection{Quadratura de Gauss-Hermite Unidimensional}

O método da quadratura de Gauss-Hermite unidimensional aproxima integrais da forma

$$
\int_{-\infty}^{\infty} g(x) \exp \left(-x^{2}\right) d x
$$

por uma soma ponderada da seguinte forma

$$
\int_{-\infty}^{\infty} g(x) \exp \left(-x^{2}\right) d x \approx \sum_{k=1}^{Q} \omega_{k} g\left(x_{k}\right)
$$

em que $g(x)$, com $x \in \mathbb{R}$ é uma função regular e o nó $x_{k}$ é o $k$-ésimo zero do polinômio de Hermite de ordem $Q, H_{Q}(x)$,

$$
H_{Q}(x)=(-1)^{Q} \exp \left(x^{2} / 2\right) \frac{d^{Q} \exp \left(-x^{2} / 2\right)}{d x^{Q}},
$$

e os pesos $\omega_{k}$ dependem do número de nós $Q$, e do polinômio de Hermite $H_{Q-1}(x)$ avaliado em $x_{k}$,

$$
\omega_{k}\left(x_{k}\right)=\frac{2^{Q-1} Q ! \sqrt{\pi}}{Q^{2}\left(H_{Q-1}\left(x_{k}\right)\right)^{2}} .
$$

\subsubsection{Quadratura de Gauss-Hermite Multidimensional}

No método da quadratura de Gauss-Hermite multidimensional considera-se o vetor de dimensão $m, \mathbf{x}=\left(x_{1}, \ldots, x_{m}\right)$, uma função regular $f(\mathbf{x}): \mathbb{R}^{m} \rightarrow \mathbb{R}$, uma função de peso $\omega(\mathbf{x}): \mathbb{R}^{m} \rightarrow \mathbb{R}^{+}$, e outra função $g(\mathbf{x}): \mathbb{R}^{m} \rightarrow \mathbb{R}$, tal que $f(\mathbf{x})=\omega(\mathbf{x}) g(\mathbf{x})$. A expressão (2.24) pode ser usada para aproximar a integral $m$-dimensional

$$
\int_{\mathbb{R}^{m}} f(\mathbf{x}) d \mathbf{x}=\int_{\mathbb{R}} \cdots \int_{\mathbb{R}} \omega\left(x_{1}, \ldots, x_{m}\right) g\left(x_{1}, \ldots, x_{m}\right) d x_{1} \cdots d x_{m},
$$

em que a função de peso é dada por

$$
\omega\left(x_{1}, \ldots, x_{m}\right)=\exp \left(-\mathbf{x}^{\top} \mathbf{x}\right)=\exp \left(-x_{1}^{2}\right) \cdots \exp \left(-x_{m}^{2}\right)=\exp \left(-\sum_{i=1}^{m} x_{i}^{2}\right) .
$$

A quadratura de Gauss-Hermite unidimensional da forma (2.24) pode ser aplicada a cada um dos componentes de $\mathbf{x}$.

$$
\int_{\mathbb{R}^{m}} f(\mathbf{x}) d \mathbf{x}=\sum_{k_{1}=1}^{Q_{1}} \omega_{k_{1}}^{(1)} \cdots \sum_{k_{m}=1}^{Q_{m}} \omega_{k_{m}}^{(m)} g\left(x_{k_{1}}^{(1)}, \ldots, x_{k_{m}}^{(m)}\right)
$$

em que $x_{k_{1}}^{(1)}$ é o $k_{r}$-ésimo zero do polinômio de Hermite de ordem $Q_{r}$, e $\omega_{k_{r}}^{(r)}, r=1, \ldots, m$ é o peso correspondente. Neste trabalho iremos denotar os $x_{k}$ como os pontos da quadratura e $Q$ como o número de pontos da quadratura. Mais detalhes sobre a quadratura de Gauss-Hermite podem ser vistos em Evans e Swartz (2000) e Fahrmeir e Tutz (2001) por exemplo. 


\section{Capítulo 3}

\section{Extensões do Modelo $t$-Potência}

\subsection{Introdução}

Nos últimos anos, houve um interesse considerável na literatura estatística em relação a famílias flexíveis de distribuições capazes de modelar dados que apresentam alto ou baixo grau de assimetria, com curtose maior ou menor do que o modelo normal usual. Neste contexto, dois modelos considerados que têm mostrado um comportamento promissor em cenários não padronizados são a distribuição SN de Azzalini (1985); e a distribuição PN por Durrans (1992).

As duas principais características dos modelos SN e PN (maior intervalo de assimetria no modelo SN e maior grau de curtose no modelo PN com matriz de informação não singular) foram combinados por Martínez-Flórez et al. (2014) num novo modelo chamado de normal-assimétricopotência e denotado PSN (power-skew-normal). Esse novo modelo inclui como casos particulares os modelos SN e PN e é capaz de modelar dados con maior ou menor grau de assimetria e curtose do que as distribuições SN e PN.

Outro conjunto de distribuições com matrizes de informação não singular, úteis para modelar dados assimétricos e de caudas pesadas são baseados em generalizações da distribuição $t$ de Student, veja por exemplo Azzalini e Capitanio (2003); Branco e Dey (2001); Gupta (2003); Sahu et al. (2003) e Jones e Faddy (2003).

Recentemente, Zhao e Kim (2016) propõem a família de distribuições chamada de $t$-potência, como alternativa à distribuição ST de (Azzalini e Capitanio, 2003) para modelar dados com caudas ainda mais pesadas e coeficiente de curtose maior do que o modelo ST.

Neste capítulo propomos algumas extensões do modelo PT para as situações em que os dados apresentam censura ou truncamento, com a possibilidade de incluir covariáves no modelo. Os novos modelos são particularmente atraentes devido ao fato que têm matriz de informação não singular e são capazes de ajustar dados com grau de assimetria e curtose maiores (ou menores) do que os modelos ST e PT.

O plano a seguir neste capítulo é como segue. Inicialmente na Seção 3.2 propomos o modelo $t$-assimétrico-potência para modelar dados com alto (ou baixo) grau de assimetria, e coeficiente de curtose maior (ou menor) do que os modelos expostos acima podem apresentar. Apresentamos também um procedimento de estimação dos parâmetros e estudamos o caso da versão de locaçãoescala do modelo. Este modelo inclui como caso particular o modelo normal-assimétrico-potência (veja Martínez-Flórez et al., 2014). Na Seção 3.3 ilustramos o nosso modelo usando um conjunto de dados sob altitudes de vulcões no mundo. Na Seção 3.4 introduzimos o modelo $t$-potência para dados censurados e truncados, apresentamos os elementos das matrizes de informação observadas dos modelos propostos. Na Seção 3.5 o modelo de regressão $t$-potência para dados censurados é introduzido. Este modelo generaliza o modelo de regressão $t$ de Student censurado estudado em detalhe por Arellano-Valle et al. (2012), e o amplamente conhecido modelo Tobit (Tobin, 
1958). Na Seção 3.6 propomos a metodologia para a análise de resíduos e a seleção do modelo. Na Seção 3.7 apresentamos um estudo de simulação em que mostramos que o método de estimação proposto oferece boas propriedades assintóticas dos estimadores dos parâmetros. Uma aplicação da utilidade do modelo introduzido a um conjuto de dados sob Salários de mulheres é apresentado na Seção 3.8. Finalmente, na Seção 3.9 discutimos as principais conlusões dos métodos propostos.

\subsection{Modelo $t$-Assimétrico-Potência}

Definição 7 Dizemos que a variável aleatória $X$ tem uma distribuição t-assimétrica-potência com parâmetro de assimetria $\lambda$, parâmetro de forma $\alpha$, e graus de liberdade $\nu$, denotada por $\operatorname{PST}(\lambda, \alpha, \nu)$, se sua fdp é dada por

$$
f_{P S T}(x \mid \lambda, \alpha, \nu)=\alpha f_{S T}(x \mid \lambda, \nu)\left[F_{S T}(x \mid \lambda, \nu)\right]^{\alpha-1}, \quad x \in \mathbb{R}, \lambda \in \mathbb{R}, \alpha \in \mathbb{R}^{+}
$$

em que $f_{S T}(\cdot)$ denota a $f d p$ da distribuição t-assimétrica padronizada com $\nu$ graus de liberdade $\operatorname{ST}(\lambda)$, e $F_{S T}(\cdot)$ é a sua fda associada.

Como casos especiais do modelo (3.1) temos que para $\alpha=1$, se segue o modelo ST, $f_{P S T}(x \mid$ $\lambda, \alpha=1, \nu) \equiv f_{S T}(x \mid \lambda, \nu)$. O modelo PT com fdp $f_{P S T}(x \mid \lambda=0, \alpha, \nu) \equiv f_{P T}(x \mid \alpha, \nu)$ segue quando $\lambda=0$. A distribuição $t$ de Student é também um caso especial a qual segue tomando $\alpha=1$ e $\lambda=0$, isto é, $f_{P S T}(x \mid \lambda=0, \alpha=1, \nu) \equiv f_{T}(x \mid \nu)=t(x \mid \nu)$. Quando $\nu \rightarrow+\infty$, a fdp da variável aleatória $X$ converge para a fdp da distribuição normal-assimétrica-potência introduzida por Martínez-Flórez et al. (2014), denotada por $\operatorname{PSN}(x ; \lambda, \alpha)$, isto é, $f_{P S T}(x \mid \lambda, \alpha, \nu \rightarrow+\infty) \equiv$ $f_{P S N}(x \mid \lambda, \alpha)$, em que

$$
f_{P S N}(x \mid \lambda, \alpha)=\alpha f_{S N}(x \mid \lambda)\left[F_{S N}(x \mid \lambda)\right]^{\alpha-1}, \quad x \in \mathbb{R}, \alpha \in \mathbb{R}^{+}
$$

$\operatorname{com} f_{S N}(\cdot)$ sendo a densidade da normal-assimétrica padronizada, e $F_{S N}(\cdot)$ é a sua fda associada.

Note-se também que os modelos SN e PN são casos particulares do modelo (3.1) tomando $\alpha=1$ e $\nu \rightarrow+\infty\left(f_{P S T}(x \mid \lambda, \alpha=1, \nu \rightarrow+\infty) \equiv f_{S N}(x \mid \lambda)\right)$; e $\lambda=0$ e $\nu \rightarrow+\infty\left(f_{P S T}(x \mid \lambda=\right.$ $\left.0, \alpha, \nu \rightarrow+\infty) \equiv f_{P N}(x \mid \alpha)\right)$ respectivamente. Finalmente, o modelo normal é obtido fazendo $\alpha=1, \lambda=0$ e $\nu \rightarrow+\infty\left(f_{P S T}(x \mid \lambda=0, \alpha=1, \nu \rightarrow+\infty) \equiv f_{N}(x) \equiv N(0,1)\right)$.

A Figura 3.1 mostra o comportamento da distribuição PST para diferentes valores de $\lambda$ e $\alpha$ quando $\nu=6$. Pode-se observar que os parâmetros $\alpha$ e $\lambda$ afetam a assimetria e a curtose da distribuição, com o qual se obtem um modelo mais flexível que os obtidos por Azzalini e Capitanio (2003) e Zhao e Kim (2016).

O $r$-ésimo momento de uma variável $X \sim \operatorname{PST}(\lambda, \alpha, \nu)$ não tem forma fechada, mas com uma mudança de variável pode ser obtido como

$$
\mathrm{E}\left[X^{r}\right]=\mu_{r}=\alpha \int_{0}^{1}\left[F_{S T}^{-1}(x \mid \lambda, \nu)\right]^{r} x^{\alpha-1} d x
$$

em que $F_{S T}^{-1}(\cdot)$ denota a inversa da função $F_{S T}(\cdot)$. Os momentos centrais, $\mu_{r}^{\prime}=\mathrm{E}\left[(X-\mathrm{E}[X])^{r}\right]$, para $r=2,3,4$ podem ser obtidos usando $\mu_{2}^{\prime}=\mu_{2}-\mu_{1}^{2}, \mu_{3}^{\prime}=\mu_{3}-3 \mu_{2} \mu_{1}+2 \mu_{1}^{3}$ e $\mu_{4}^{\prime}=\mu_{4}-$ $4 \mu_{3} \mu_{1}+6 \mu_{2} \mu_{1}^{2}-3 \mu_{1}^{4}$. Portanto, a variância, o coeficiente de variação, e coeficientes de assimetria e curtose podem ser obtidos como: $\operatorname{Var}[X]=\sigma_{X}^{2}=\mu_{2}^{\prime}, \mathrm{CV}=\sqrt{\mu_{2}^{\prime}} / \mu_{1}, \sqrt{\beta_{1}}=\mu_{3}^{\prime} /\left(\mu_{2}^{\prime}\right)^{3 / 2} \mathrm{e}$ $\beta_{2}=\mu_{4}^{\prime} /\left(\mu_{2}^{\prime}\right)^{2}$, respectivamente.

A Tabela 3.1 apresenta o intervalo de possíveis valores para os coeficientes de assimetria e curtose para os modelos $\operatorname{ST}(\lambda, \nu), \operatorname{PT}(\alpha, \nu)$ e $\operatorname{PST}(\lambda, \alpha, \nu)$, com valores de $\lambda$ entre -40 e 40 , valores de $\alpha$ de 0.5 a 50 , e valores de $\nu=2,3,4,5,6,7$. Os cálculos foram feitos usando a função 


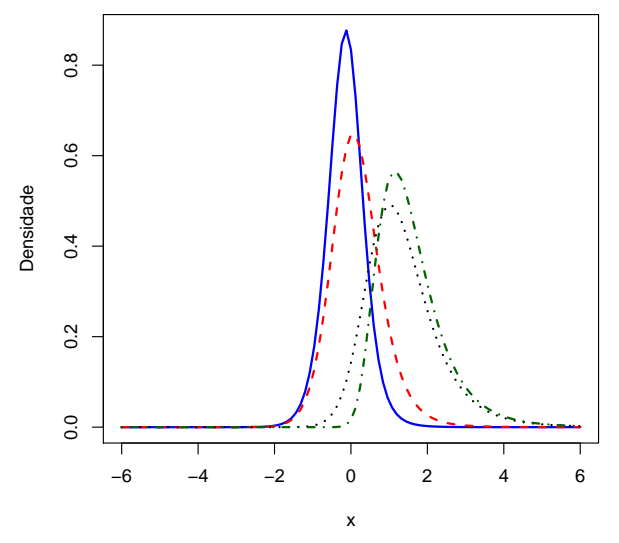

(a)

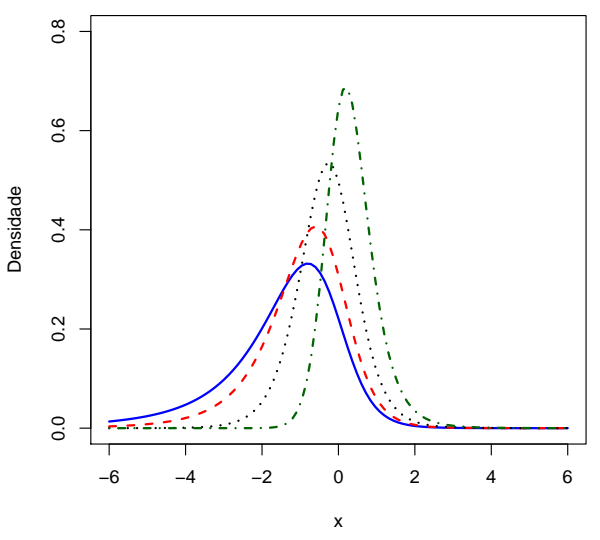

(b)

Figura 3.1: Função densidade de probabilidade de: (a) Distribuição $P S T(\lambda, 2.5,6)$ para $\lambda=-2.0$ (linha sólida), $\lambda=-1.0$ (linha tracejada), $\lambda=0.5$ (linha pontilhada), $\lambda=2.5$ (linha pontilhada-tracejada). (b) Distribuição $\operatorname{PST}(-1, \alpha, 6)$ para $\alpha=0.5$ (linha sólida), $\alpha=0.75$ (linha tracejada), $\alpha=1.5$ (linha pontilhada), $\alpha=4$ (linha pontilhada-tracejada).

integrate do pacote estatístico R (R Development Core Team, 2017).

Pode-se observar da Tabela 3.1 que o intervalo de valores dos coeficientes de assimetria e curtose do modelo PST, contêm os respectivos intervalos de valores dos coeficientes de assimetria e curtose dos modelos ST e PT. Isto é um indicador de que o modelo PST é mais flexível em termos de assimetria e curtose que os modelos ST e PT.

\subsubsection{Estimação}

No modelo PST consideramos o método de máxima verossimilhança para realizar a inferência do vetor de parâmetros $\boldsymbol{\theta}=(\lambda, \alpha, \nu)^{\top}$. Assim, dada uma amostra aleatória $\mathbf{X}=\left(X_{1}, \ldots, X_{n}\right)$ $\operatorname{com} X_{i} \sim \operatorname{PST}(\lambda, \alpha, \nu)$, temos que a função de verossimilhança para o vetor $\boldsymbol{\theta}$ é dada por

$$
\begin{aligned}
L(\boldsymbol{\theta} ; \mathbf{X})=(2 \alpha)^{n}(\pi \nu)^{-\frac{n}{2}}\left\{\frac{\Gamma\left(\frac{\nu+1}{2}\right)}{\Gamma\left(\frac{\nu}{2}\right)}\right\}^{n} \\
\quad \times \prod_{i=1}^{n}\left\{1+\frac{x_{i}^{2}}{\nu}\right\}^{-\left(\frac{\nu+1}{2}\right)} T\left(\lambda x_{i} \sqrt{\frac{\nu+1}{x_{i}^{2}+\nu}} \mid \nu+1\right)\left\{F_{S T}\left(x_{i} \mid \lambda, \nu\right)\right\}^{\alpha-1}
\end{aligned}
$$

e a função de log-verossimilhança obtida de (3.4) é

$$
\begin{aligned}
\ell(\boldsymbol{\theta} ; \mathbf{X})=\text { cte } & +n \log \alpha-\frac{n}{2} \log \nu+n \log \Gamma\left(\frac{\nu+1}{2}\right) \\
& -n \log \Gamma\left(\frac{\nu}{2}\right)-\frac{\nu+1}{2} \sum_{i=1}^{n} \log \left(1+\frac{x_{i}^{2}}{\nu}\right) \\
+ & \sum_{i=1}^{n} \log T\left(\lambda x_{i} w_{i} \mid \nu+1\right)+(\alpha-1) \sum_{i=1}^{n} \log F_{S T}\left(x_{i} \mid \lambda, \nu\right)
\end{aligned}
$$


Tabela 3.1: Intervalo de valores para os coeficientes de assimetria e curtose dos modelos $\operatorname{ST}(\lambda, \nu)$, $\operatorname{PT}(\alpha, \nu)$ e $\operatorname{PST}(\lambda, \alpha, \nu)$ baseados num estudo de simulação. Valores de $\lambda$ de -40 a $40, \alpha$ de 0.5 a 50 , e $\nu=2,3,4,5,6,7$.

\begin{tabular}{llrrrrrr}
\hline & & \multicolumn{2}{c}{ Modelo ST } & \multicolumn{2}{c}{ Modelo PT } & \multicolumn{2}{c}{ Modelo PST } \\
$\nu$ & Amplitude & Assimetria & Curtose & Assimetria & Curtose & Assimetria & Curtose \\
\hline 2 & Min. & -0.9625 & 3.1698 & -0.1190 & 1.5521 & -2.4521 & 1.3951 \\
& Max. & 0.9625 & 3.4883 & 3.0395 & 10.4355 & 14.3140 & 864.3852 \\
3 & Min. & -0.9503 & 3.1460 & -0.0855 & 1.3245 & -2.1298 & 1.6282 \\
& Max. & 0.9503 & 3.3573 & 1.3621 & 3.2226 & 4.9023 & 114.0981 \\
4 & Min. & -1.8526 & 5.0989 & -0.5303 & 3.4606 & -1.8984 & 3.1525 \\
& Max. & 1.8526 & 7.8239 & 1.1783 & 5.2986 & 3.2146 & 29.8740 \\
5 & Min. & -0.9474 & 3.0507 & -0.4753 & 1.1756 & -1.9682 & 3.8623 \\
& Max. & 0.9474 & 3.3266 & 0.2712 & 3.1300 & 3.0461 & 19.9245 \\
6 & Min. & -1.6812 & 4.5543 & -0.5330 & 3.9737 & -1.6812 & 3.8919 \\
& Max. & 1.6812 & 7.2786 & 1.1180 & 5.1733 & 2.1446 & 11.8930 \\
7 & Min. & -0.9443 & 3.0068 & -0.7104 & 1.2635 & -1.5349 & 3.1358 \\
& Max. & 0.9443 & 3.3674 & 0.2430 & 3.0821 & 2.5362 & 15.9235 \\
\hline
\end{tabular}

em que $w=\sqrt{\frac{\nu+1}{x^{2}+\nu}}$. A Figura 3.2 mostra os perfis da função de log-verossimilhança para $\alpha$ variando de 0.1 a 6 para amostras simuladas de uma distribuição $\operatorname{PST}(-2, \alpha, 5)$, o perfil da função de log-verossimilhança para $\lambda$ tomando valores de -6 a 6 para amostras simuladas de uma distribuição $\operatorname{PST}(\lambda, 2.5,10)$ e o perfil da função de log-verossimilhança para $\nu$ tomando valores de 0.9 a 40 para amostras simuladas de uma distribuição $\operatorname{PST}(1.5,0.5, \nu)$. Os tamanhos de amostras são 50, 100 e 200.

Definindo $r(x \mid \nu)=t(x \mid \nu) / T(x \mid \nu)$ e $R_{\lambda}(x \mid \nu)=\left(1+\left(1+\lambda^{2}\right) x^{2} / \nu\right)^{-\frac{\nu}{2}} / F_{S T}(x \mid \lambda, \nu)$, temos que os componentes da função escore $U_{\theta_{i}}(\boldsymbol{\theta})$ são obtidos derivando parcialmente $\ell(\boldsymbol{\theta} ; X)$ em (3.5), com respecto a $\lambda, \alpha$ e $\nu$, e ficam dados por

$$
\begin{aligned}
& U_{\lambda}(\boldsymbol{\theta})=\sum_{i=1}^{n} x_{i} w_{i} r\left(\lambda x_{i} w_{i} \mid \nu+1\right)-\frac{\alpha-1}{\pi\left(1+\lambda^{2}\right)} \sum_{i=1}^{n} R_{\lambda}\left(x_{i} \mid \nu\right), \\
& U_{\alpha}(\boldsymbol{\theta})=\frac{n}{\alpha}+\sum_{i=1}^{n} \log F_{S T}\left(x_{i} \mid \lambda, \nu\right),
\end{aligned}
$$



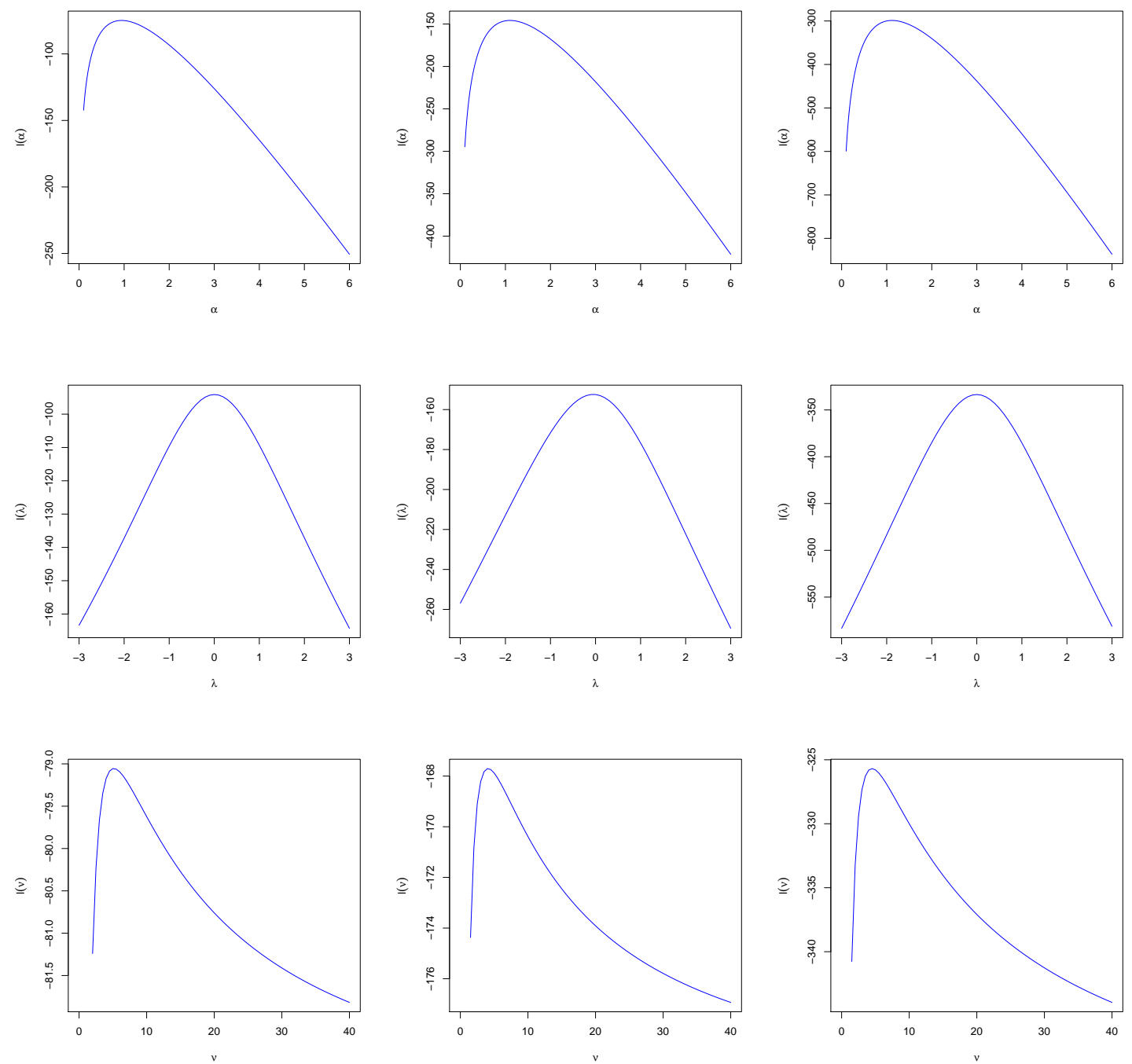

Figura 3.2: Perfil da função de log-verossimilhança $\ell_{p}(\alpha)$ (primeira linha), $\ell_{p}(\lambda)$ (segunda linha) e $\ell_{p}(\nu)$ (terceira linha). Tamanhos de amostras $n=50$ (primeira coluna), $n=100$ (segunda coluna), e $n=200$ (terceira coluna).

$$
\begin{aligned}
U_{\nu}(\boldsymbol{\theta})=\frac{n \alpha}{2} & \left(\psi\left(\frac{\nu+1}{2}\right)-\psi\left(\frac{\nu}{2}\right)-\frac{1}{\nu}\right)-\frac{1}{2} \sum_{i=1}^{n} \log \left(1+\frac{x_{i}^{2}}{\nu}\right) \\
& +\frac{\nu+1}{2 \nu} \sum_{i=1}^{n} \frac{x_{i}^{2}}{\nu}\left(1+\frac{x_{i}^{2}}{\nu}\right)^{-1} \\
& +\frac{\lambda}{2(\nu+1)} \sum_{i=1}^{n} \frac{x_{i}^{3}}{\nu}\left(1+\frac{x_{i}^{2}}{\nu}\right)^{-1} w_{i} r\left(\lambda x_{i} w_{i} \mid \nu+1\right) \\
& -\frac{\lambda}{2(\nu+1)} \sum_{i=1}^{n} \frac{x_{i}}{\nu}\left(1+\frac{x_{i}^{2}}{\nu}\right)^{-1} w_{i} r\left(\lambda x_{i} w_{i} \mid \nu+1\right) \\
& -\frac{(\alpha-1)}{2 \pi(\nu+1)} \frac{\lambda}{\left(1+\lambda^{2}\right)} \sum_{i=1}^{n} R_{\lambda}\left(x_{i} \mid \nu\right) \\
& +\frac{\alpha-1}{2} \sum_{i=1}^{n} \frac{g\left(x_{i} ; \nu\right)}{F_{S T}\left(x_{i} \mid \lambda, \nu\right)}
\end{aligned}
$$


em que $\psi(x)=\frac{d}{d x} \log \Gamma(x)$ é a função digama, e

$$
\begin{gathered}
g(x ; \nu)=\int_{-\infty}^{x}\left\{\frac{(\nu+1) s^{2}}{\nu\left(s^{2}+\nu\right)}-\log \left(1+\frac{s^{2}}{\nu}\right)\right\} f_{S T}(s \mid \lambda, \nu) d s \\
-\frac{\lambda}{\pi} \int_{-\infty}^{x} \frac{s}{s^{2}+\nu}\left\{1+\frac{\left(1+\lambda^{2}\right) s^{2}}{\nu}\right\}^{-\frac{\nu+2}{2}} d s
\end{gathered}
$$

Note-se que, se $\nu \rightarrow \infty$, então $U_{\nu}(\boldsymbol{\theta}) \rightarrow 0$ e as equações (3.6) e (3.7) se reduzem às funções escore da distribuição normal-assimétrica-potência (veja Martínez-Flórez et al., 2014), as quais são dadas por

$$
U_{\lambda}(\boldsymbol{\theta})=\sum_{i=1}^{n} x_{i} \frac{\phi\left(\lambda x_{i}\right)}{\Phi\left(\lambda x_{i}\right)}-\sqrt{\frac{2}{\pi}} \frac{(\alpha-1)}{1+\lambda^{2}} \sum_{i=1}^{n} \frac{\phi\left(\sqrt{1+\lambda^{2}} x_{i}\right)}{F_{S N}\left(x_{i} \mid \lambda\right)}
$$

e

$$
U_{\alpha}(\boldsymbol{\theta})=\frac{n}{\alpha}+\sum_{i=1}^{n} \log F_{S N}\left(x_{i} \mid \lambda\right)
$$

O estimador de máxima verosimilhança para $\boldsymbol{\theta}=(\lambda, \alpha, \nu)^{\top}$ é obtido usando procedimentos iterativos baseados no algoritmo de Newton-Raphson usando as funções escore (3.6) - (3.8).

\subsubsection{Matriz de Informação}

Os elementos da matriz de informação observada $\boldsymbol{J}(\boldsymbol{\theta})$, denotados por $j_{\boldsymbol{\theta}_{k} \boldsymbol{\theta}_{i}}$, são obtidos depois de computar a segunda derivada parcial da função de log-verossimilhança (3.5) em relação a cada um dos parâmetros e multiplicar por -1, isto é, $j_{\boldsymbol{\theta}_{k} \boldsymbol{\theta}_{i}}=-\partial^{2} \ell(\boldsymbol{\theta} ; \mathbf{X}) / \partial \boldsymbol{\theta}_{k} \partial \boldsymbol{\theta}_{i}$. Estes elementos são dados no Apêndice B.1.1. As estimativas das variâncias dos estimadores podem ser obtidas avaliando a inversa da matriz de informação observada $\boldsymbol{J}^{-1}(\boldsymbol{\theta})$ no estimador de máxima verossimilhança $\widehat{\boldsymbol{\theta}}=(\widehat{\lambda}, \widehat{\alpha}, \widehat{\nu})^{\top}$.

Sob as condições usuais de regularidade, o estimador de máxima verossimilhança $\widehat{\boldsymbol{\theta}}$ é assintóticamente normal com média $\boldsymbol{\theta}$ e matriz de covariâncias $\boldsymbol{I}^{-1}(\boldsymbol{\theta})$, isto é,

$$
\sqrt{n}(\widehat{\boldsymbol{\theta}}-\boldsymbol{\theta}) \rightarrow N\left(\mathbf{0}, \boldsymbol{I}^{-1}(\boldsymbol{\theta})\right)
$$

em que $\boldsymbol{I}(\boldsymbol{\theta})$ é a matriz de informação esperada, obtida como $\boldsymbol{I}(\boldsymbol{\theta})=\mathrm{E}[\boldsymbol{J}(\boldsymbol{\theta})]$.

\subsubsection{Modelo PST de Locação e Escala}

Considerando uma variável aleatória $Z$ com distribuição $\operatorname{PST}(\lambda, \alpha, \nu)$, a extensão para locaçãoescala de $Z$ pode ser obtida usando a transformação $X=\xi+\eta Z$, em que $\xi \in \mathbb{R}$ e $\eta \in \mathbb{R}^{+}$. A fdp de $Z$ é dada por

$$
f_{P S T}(x \mid \xi, \eta, \lambda, \alpha, \nu)=\alpha f_{S T}(x \mid \xi, \eta, \lambda, \nu)\left[F_{S T}(x \mid \xi, \eta, \lambda, \nu)\right]^{\alpha-1}, x \in \mathbb{R}, \alpha \in \mathbb{R}^{+},
$$

em que

$$
f_{S T}(x \mid \xi, \eta, \lambda, \nu)=\frac{2}{\eta} t\left(\frac{x-\xi}{\eta} \mid \nu\right) T\left(\lambda\left(\frac{x-\xi}{\eta}\right) \sqrt{\frac{\nu+1}{Q_{x}+\nu}} \mid \nu+1\right), \quad x \in \mathbb{R}, \lambda \in \mathbb{R},
$$

$\mathrm{e}$

$$
F_{S T}(x \mid \xi, \eta, \lambda, \nu)=\int_{-\infty}^{x} f_{S T}(s \mid \xi, \eta, \lambda, \nu) d s
$$


Escreveremos esta extensão por $X \sim \operatorname{PST}(\xi, \eta, \lambda, \alpha, \nu)$, portanto $\operatorname{PST}(0,1, \lambda, \alpha, \nu) \equiv \operatorname{PST}(\lambda, \alpha, \nu)$, e temos que se $X \sim \operatorname{PST}(\xi, \eta, \lambda, \alpha, \nu)$, a fda de $X$ é dada por

$$
F_{P S T}(x \mid \xi, \eta, \lambda, \alpha, \nu)=\left[F_{S T}(x \mid \xi, \eta, \lambda, \nu)\right]^{\alpha}, \quad x \in \mathbb{R}, \alpha \in \mathbb{R}^{+} .
$$

O método da transformação inversa pode ser usado para gerar uma variável aleatória $X \sim$ $\operatorname{PST}(\xi, \eta, \lambda, \alpha, \nu)$ de (3.13), a partir de uma variável aleatória $U$ com distribuição uniforme $\mathrm{U}(0,1)$, e aplicando a relação

$$
X=\xi+\eta \times F_{S T}^{-1}\left(U^{1 / \alpha} \mid \lambda, \nu\right)
$$

em que $F_{S T}^{-1}(\cdot \mid \lambda, \nu)$ é a inversa da função $F_{S T}(\cdot \mid \lambda, \nu)$, (isto é, a fda da distribuição ST padrão com $\nu$ graus de liberdade).

O $r$-ésimo momento de uma variável aleatória $X \sim \operatorname{PST}(\xi, \eta, \lambda, \alpha, \nu)$ pode ser calculado como

$$
\mathrm{E}\left[X^{r}\right]=\sum_{k=0}^{r}\left(\begin{array}{l}
r \\
k
\end{array}\right) \xi^{k} \eta^{r-k} \mu_{r-k},
$$

em que $\mu_{j}$, é o $j$-ésimo momento de uma variável aleatória $Z \sim \operatorname{PST}(\lambda, \alpha, \nu)$ para $j=1,2, \ldots$, e $\mu_{0}=1$. O processo de inferência é realizado usando o método da máxima verossimilhança. Temos que, dada uma amostra aleatória $\mathbf{X}=\left(X_{1}, \ldots, X_{n}\right) \operatorname{com} X_{i} \sim \operatorname{PST}(\xi, \eta, \lambda, \alpha, \nu)$, a função de $\log$-verossimilhança para o vetor de parâmetros $\boldsymbol{\theta}=(\xi, \eta, \lambda, \alpha, \nu)^{\top}$ é dado por

$$
\begin{aligned}
\ell(\boldsymbol{\theta} ; \mathbf{X})=\text { cte } & +n \log (\alpha)-n \log (\eta)-\frac{n}{2} \log (\nu) \\
& +n \log \Gamma\left(\frac{\nu+1}{2}\right)-n \log \Gamma\left(\frac{\nu}{2}\right)-\frac{\nu+1}{2} \sum_{i=1}^{n} \log \left(1+\frac{z_{i}^{2}}{\nu}\right) \\
+ & \sum_{i=1}^{n} \log T\left(\lambda z_{i} \sqrt{\frac{\nu+1}{z_{i}^{2}+\nu}} ; \nu+1\right)+(\alpha-1) \sum_{i=1}^{n} \log F_{S T}\left(z_{i} \mid \lambda, \nu\right)
\end{aligned}
$$

em que $z_{i}=\left(x_{i}-\xi\right) / \eta$. A função escore é dada por

$$
\boldsymbol{U}(\boldsymbol{\theta})=\left(U_{\xi}(\boldsymbol{\theta}), U_{\eta}(\boldsymbol{\theta}), U_{\lambda}(\boldsymbol{\theta}), U_{\alpha}(\boldsymbol{\theta}), U_{\nu}(\boldsymbol{\theta})\right)^{\top}
$$

e seus componentes $U_{\theta_{i}}(\boldsymbol{\theta})$ são obtidos de (3.15) derivando parcialmente $\ell(\boldsymbol{\theta} ; \mathbf{X})$ com relação a $\xi, \eta, \lambda, \alpha$ e $\nu$ dados por 


$$
\begin{aligned}
& U_{\xi}(\boldsymbol{\theta})=\frac{\nu+1}{\eta} \sum_{i=1}^{n} \frac{z_{i}}{\nu}\left(1+\frac{z_{i}^{2}}{\nu}\right)^{-1} \\
& -\frac{\lambda \nu}{\eta} \sum_{i=1}^{n} \frac{w_{i}}{\nu}\left(1+\frac{z_{i}^{2}}{\nu}\right)^{-1} r\left(\lambda z_{i} w_{i} \mid \nu+1\right) \\
& -\frac{\alpha-1}{\eta} \sum_{i=1}^{n} r_{\lambda}\left(z_{i} \mid \nu\right) \\
& U_{\eta}(\boldsymbol{\theta})=-\frac{n}{\eta}+\frac{\nu+1}{\eta} \sum_{i=1}^{n} \frac{z_{i}^{2}}{\nu}\left(1+\frac{z_{i}^{2}}{\nu}\right)^{-1} \\
& -\frac{\lambda \nu}{\eta} \sum_{i=1}^{n} \frac{z_{i} w_{i}}{\nu}\left(1+\frac{z_{i}^{2}}{\nu}\right)^{-1} r\left(\lambda z_{i} w_{i} \mid \nu+1\right) \\
& -\frac{\alpha-1}{\eta} \sum_{i=1}^{n} z_{i} r_{\lambda}\left(z_{i} \mid \nu\right) \\
& U_{\lambda}(\boldsymbol{\theta})=\sum_{i=1}^{n} z_{i} w_{i} r\left(\lambda z_{i} w_{i} \mid \nu+1\right) \\
& -\frac{\alpha-1}{\pi\left(1+\lambda^{2}\right)} \sum_{i=1}^{n} R_{\lambda}\left(z_{i} \mid \nu\right) \\
& U_{\alpha}(\boldsymbol{\theta})=\frac{n}{\alpha}+\sum_{i=1}^{n} \log \Phi_{\lambda, \nu}\left(z_{i}\right) \\
& U_{\nu}(\boldsymbol{\theta})=\frac{n \alpha}{2}\left(\psi\left(\frac{\nu+1}{2}\right)-\psi\left(\frac{\nu}{2}\right)-\frac{1}{\nu}\right)-\frac{1}{2} \sum_{i=1}^{n} \log \left(1+\frac{z_{i}^{2}}{\nu}\right) \\
& +\frac{\nu+1}{2 \nu} \sum_{i=1}^{n} \frac{z_{i}^{2}}{\nu}\left(1+\frac{z_{i}^{2}}{\nu}\right)^{-1} \\
& +\frac{\lambda}{2(\nu+1)} \sum_{i=1}^{n} \frac{z_{i}^{3}}{\nu}\left(1+\frac{z_{i}^{2}}{\nu}\right)^{-1} w_{i} r\left(\lambda z_{i} w_{i} \mid \nu+1\right) \\
& -\frac{\lambda}{2(\nu+1)} \sum_{i=1}^{n} \frac{z_{i}}{\nu}\left(1+\frac{z_{i}^{2}}{\nu}\right)^{-1} w_{i} r\left(\lambda z_{i} w_{i} \mid \nu+1\right) \\
& -\frac{(\alpha-1)}{2 \pi(\nu+1)} \frac{\lambda}{\left(1+\lambda^{2}\right)} \sum_{i=1}^{n} R_{\lambda}\left(z_{i} \mid \nu\right)+\frac{\alpha-1}{2} \sum_{i=1}^{n} \frac{g\left(z_{i} ; \nu\right)}{F_{S T}\left(z_{i} \mid \lambda, \nu\right)}
\end{aligned}
$$

em que $\psi(z)$ é a função digama, $w=\sqrt{\frac{\nu+1}{z^{2}+\nu}}, r(z \mid \nu)=t(z \mid \nu) / T(z \mid \nu), r_{\lambda}(z \mid \nu)=f_{S T}(z \mid$ $\lambda, \nu) / F_{S T}(z \mid \lambda, \nu), R_{\lambda}(x \mid \nu)=\left(1+\left(1+\lambda^{2}\right) z^{2} / \nu\right)^{-\frac{\nu}{2}} / F_{S T}(z \mid \lambda, \nu)$ e $g(z ; \nu)$ é a função definida em (3.9).

De novo como no modelo PST padronizado, os elementos da matriz de informação observada $\boldsymbol{J}(\boldsymbol{\theta})$, denotados por $j_{\boldsymbol{\theta}_{k} \boldsymbol{\theta}_{i}}$, são obtidos depois de computar a segunda derivada parcial da função de $\log$-verossimilhança (3.15) e multiplicar por -1, isto é, $j_{\boldsymbol{\theta}_{k} \boldsymbol{\theta}_{i}}=-\partial^{2} \ell(\boldsymbol{\theta} ; \mathbf{X}) / \partial \boldsymbol{\theta}_{k} \partial \boldsymbol{\theta}_{i}$. Estes elementos são dados no Apêndice B.1.2. Pode-se mostrar que para $\lambda=0$ e $\alpha=1$, as matrizes de informação observada e esperada, a qual é obtida como $\boldsymbol{I}(\boldsymbol{\theta})=\mathrm{E}[\boldsymbol{J}(\boldsymbol{\theta})]$ são não singulares quando $\nu$ é pequeno (veja Zhao e Kim, 2016). 
Quando $\nu \rightarrow \infty$, a distribuição ST converge para a distribuição SN, e aqui nós lembramos que a matriz de informação de uma variável aleatória $X \sim \mathrm{SN}(\xi, \eta, \lambda)$ denotada por $\mathbf{I}_{\lambda}(\boldsymbol{\varphi})$, em que $\boldsymbol{\varphi}=(\xi, \eta, \lambda)^{\top}$, é singular quando $\lambda=0$. Portanto, é conveniente usar a representação centrada da ST estudada por Arellano-Valle e Azzalini (2013).

Inicialmente, a parametrização centrada da distribuição SN é uma alternativa ao problema da singularidade da matriz de informação da SN quando $\lambda=0$, assim Arellano-Valle e Azzalini (2008) propuseram uma segunda representação da distribuição SN definindo uma nova variável aleatória $X$ como

$$
X=\mu+\sigma\left(\frac{Z-\mathrm{E}[Z]}{\sqrt{\operatorname{Var}[Z]}}\right),
$$

em que $\mu \in \mathbb{R}$ e $\sigma>0$ são parâmetros da variável aleatória $X$ e $Z \sim \operatorname{SN}(\lambda)$. Essa representação é chamada de parametrização centrada, uma vez que $\mathrm{E}[X]=\mu$ e $\operatorname{Var}[X]=\sigma^{2}$ e é denotada por $\mathrm{SN}_{c}\left(\mu, \sigma, \gamma_{1}\right)$, em que $-0.9953<\gamma_{1}<0.9953$.

Sob o modelo de parametrização centrado, $\mu, \sigma$ e $\gamma_{1}=\sqrt{\beta_{1}}$ representam como de costume, a média, o desvío padrão e o coeficiente de assimetria de $X$, respectivamente. Agora, se $Z \sim \mathrm{SN}(\lambda)$ então $\mathrm{E}[Z]=b \delta$ e $\operatorname{Var}[Z]=1-(b \delta)^{2}$, em que $b=\sqrt{2 / \pi}$ e $\delta=\lambda / \sqrt{1+\lambda^{2}}$; então segue que a variável aleatória $X$ pode ser escrita na forma $X=\xi+\eta Z$ a qual tem uma distribuição $S N\left(\lambda_{1}, \lambda_{2}, \lambda\right)$, em que

$$
\lambda_{1}=\mu-c \sigma \gamma_{1}^{1 / 3}, \quad \lambda_{2}=\sigma \sqrt{1+c^{2} \gamma_{1}^{2 / 3}}, \quad \lambda=\frac{c \gamma_{1}^{1 / 3}}{\sqrt{b^{2}+c^{2}\left(b^{2}-1\right) \gamma_{1}^{2 / 3}}}
$$

com $c=\{2 /(4-\pi)\}^{1 / 3}$. Sob essa designação, temos que a matriz de informação pode ser escrita na forma $\mathbf{I}_{\gamma_{1}}=\mathbf{D}^{\top} \mathbf{I}_{\lambda} \mathbf{D}$, em que $\mathbf{D}$ é uma matriz que representa a derivada dos parâmetros da representação padrão $\left(\lambda_{1}, \lambda_{2}\right.$ e $\left.\lambda\right)$ com relação aos novos parâmetros $\left(\mu, \sigma\right.$ e $\left.\gamma_{1}\right)$. Segue também que a matriz de informação converge para a matriz diagonal $\boldsymbol{\Sigma}_{c}^{-1}=\operatorname{diag}\left(\sigma^{2}, \sigma^{2} / 2,6\right)$, quando $\lambda \rightarrow 0$. Isso garante a existência e unicidade do estimador de máxima verossimilhança (EMV) de $\lambda_{1}$ e $\lambda_{2}$ para cada valor fixo de $\lambda$.

Seguindo essa mesma linha de pensamento, nós propomos que $Y$ segue uma distribuição ST de locação-escala (3.12) com parâmetro de locação $\xi \in \mathbb{R}$ e parâmetro de escala $\eta>0$, isto é

$$
\phi_{\lambda, \nu}(y ; \xi, \eta)=\frac{2}{\eta} t\left(\frac{y-\xi}{\eta} \mid \nu\right) T\left(\lambda\left(\frac{y-\xi}{\eta}\right) \sqrt{\frac{\nu+1}{Q_{y}+\nu}} \mid \nu+1\right),
$$

para $\lambda \in \mathbb{R}$, com $Q_{y}=((y-\xi) / \eta)^{2}$ e $z=(y-\xi) / \eta$, a qual é denotada $\operatorname{ST}(\xi, \eta, \lambda, \nu)$. Essa representação faz relação com a parametrização direta da distribuição ST com vetor de parâmetro $\boldsymbol{\rho}=(\xi, \eta, \lambda, \nu)^{\top}$. Então, segue que $Z_{T}=(Y-\xi) / \eta \sim \mathrm{ST}(0,1, \lambda, \nu)$, e pela representação estocástica da distribuição ST dada por $Z_{T}=Z / \sqrt{V}$, em que $Z \sim \operatorname{SN}(0,1, \lambda)$ e $V \sim \chi_{\nu}^{2} / \nu$, Isso leva a calcular os quatro primeiros cumulantes de $Z_{T}$ denotados por $\mu_{1}(\delta, \nu), \mu_{2}(\delta, \nu), \mu_{3}(\delta, \nu) \mathrm{e}$ $\mu_{4}(\delta, \nu)$ (veja Arellano-Valle e Azzalini, 2013).

A parametrização centrada da distribuição ST de uma variável aleatória $Y$ segue da definição 
de

$$
\begin{aligned}
\mu_{t} & =\mathrm{E}(Y)=\xi+\eta \mu_{1}(\delta, \nu) \\
& =\xi+\eta b_{\nu} \delta \\
\sigma_{t}^{2} & =\operatorname{Var}(Y)=\eta^{2} \mu_{2}(\delta, \nu) \\
& =\eta^{2}\left\{\frac{\nu}{\nu-2}-b_{\nu}^{2} \delta^{2}\right\} \\
\gamma_{1 t} & =\frac{\mu_{3}(\delta, \nu)}{\mu_{2}(\delta, \nu)^{3 / 2}} \\
& =\frac{b_{\nu} \delta}{\mu_{2}(\delta, \nu)^{3 / 2}}\left\{\frac{\nu\left(3-\delta^{2}\right)}{\nu-3}-\frac{3 \nu}{\nu-2}+2 b_{\nu}^{2} \delta^{2}\right\} \quad \mathrm{e} \\
\gamma_{2 t} & =\frac{\mu_{4}(\delta, \nu)}{\mu_{2}(\delta, \nu)^{2}} \\
& =\frac{1}{\mu_{2}(\delta, \nu)^{2}}\left\{\frac{3 \nu^{2}}{(\nu-2)(\nu-4)}-\frac{4 b_{\nu}^{2} \delta^{2} \nu\left(3-\delta^{2}\right)+\frac{6 b_{\nu}^{2} \delta^{2} \nu}{\nu-2}-4 b_{\nu}^{4} \delta^{4}}{\nu-3}\right\}-3 .
\end{aligned}
$$

A nova representação será definida como a distribuição $t$-assimétrica centrada com vetor de parâmetros $\overline{\boldsymbol{\rho}}=\left(\mu, \sigma^{2}, \gamma_{1}, \gamma_{2}\right)^{\top}$. De acordo com Arellano-Valle e Azzalini (2013), temos que a matriz de informação dessa representação pode ser escrita como

$$
\mathbf{I}(\overline{\boldsymbol{\rho}})=\mathbf{B}^{\top} \mathbf{I}(\boldsymbol{\rho}) \mathbf{B}
$$

em que $\mathbf{B}$ é uma matriz que representa a derivada do vetor de parâmetros $\boldsymbol{\rho}$ com respecto ao novo vetor $\overline{\boldsymbol{\rho}}$. Pode-se mostrar que $b_{\nu} \rightarrow b$ quando $\nu \rightarrow \infty$ (veja Arellano-Valle e Azzalini, 2013). Portanto, temos que os parâmetros do modelo ST centrado convergem para $\mu_{t} \rightarrow \mu, \sigma_{t}^{2} \rightarrow \sigma^{2} \mathrm{e}$ $\gamma_{1 t} \rightarrow \gamma_{1}$, quando $\nu \rightarrow \infty$, isto é, os parâmetros da $\mathrm{SN}_{c}$.

Então como $Z_{T} \rightarrow \mathrm{SN}(0,1, \lambda)$ quando $\nu \rightarrow \infty$, segue que a variável aleatória $Y$ converge para uma distribuição com matriz de informação dada por

$$
\mathbf{I}\left(\mu, \sigma^{2}, \gamma_{1}, \alpha\right)=\left(\begin{array}{cc}
\mathbf{I}_{\boldsymbol{\theta}_{1} \boldsymbol{\theta}_{1}} & \mathbf{I}_{\boldsymbol{\theta}_{1}, \alpha} \\
\mathbf{I}_{\boldsymbol{\theta}_{1}, \alpha}^{\top} & I_{\alpha, \alpha}
\end{array}\right)
$$

quando $\nu \rightarrow \infty$, em que os elementos da diagonal correspondentes à informação do vetor de parâmetros $\boldsymbol{\theta}_{1}=\left(\mu, \sigma^{2}, \gamma_{1}\right)$ e $\alpha$; e $\mathbf{I}_{\boldsymbol{\theta}_{1}, \alpha}$ é a informação conjunta de $\boldsymbol{\theta}_{1}=\left(\mu, \sigma^{2}, \gamma_{1}\right)^{\top}$ e $\alpha$. Agora, para $\lambda \rightarrow 0$ e $\alpha=1$, pode-se mostrar que $\mathbf{I}_{\boldsymbol{\theta}_{1} \boldsymbol{\theta}_{1}} \rightarrow \operatorname{diag}\left(\sigma^{2}, \sigma^{2} / 2,6\right)$, com determinante $0.3333 / \sigma^{4}$, e $\mathbf{I}_{\boldsymbol{\theta}_{1}, \alpha}=(0.9031 / \sigma,-0.5956 / \sigma, 0.7206)^{\top}$, portanto, o determinante $\left|\mathbf{I}\left(\mu, \sigma^{2}, \gamma_{1}, \alpha\right)\right| \neq 0$, isto é, as linhas (ou colunas) da matriz $\mathbf{I}\left(\mu, \sigma^{2}, \gamma_{1}, \alpha\right)$ são linearmente independentes, e se conclui que a variável aleatória $Y$ converge para uma distribuição com matriz de informação não singular quando $\nu \rightarrow \infty$.

\subsection{Aplicação}

Nesta seção, aplicamos o modelo proposto usando um conjunto de dados consistente de altitudes de 1520 vulcões do mundo. Os dados estão disponíveis em Global Volcanism Program (2013). A Tabela 3.2 mostra os coeficientes de assimetria e curtose para os dados. Note-se que os valores dos coeficientes de assimetria $\left(\sqrt{b_{1}}=0.6461\right)$ e curtose $\left(b_{2}=4.3809\right)$ justificam o uso de um modelo assimétrico e de caudas pesadas como o modelo PST, por exemplo.

O conjunto de datos é analisado ajustando un modelo $t$ de Student (T), $t$-assimétrico (ST), 
Tabela 3.2: Resumo estatístico para os dados de vulcões.

\begin{tabular}{ccccc}
\hline$n$ & Média & Variância & $\sqrt{b_{1}}$ & $b_{2}$ \\
\hline 1520 & 16.7760 & 15.6682 & 0.6461 & 4.3809 \\
\hline
\end{tabular}

Tabela 3.3: Estimativas dos parâmetros e erros padrão (EP) para os modelos T, ST, PT e PST ajustados aos dados de vulcões.

\begin{tabular}{|c|c|c|c|c|c|c|c|c|}
\hline \multirow[b]{2}{*}{$\theta$} & \multicolumn{2}{|c|}{ Modelo T } & \multicolumn{2}{|c|}{ Modelo ST } & \multicolumn{2}{|c|}{ Modelo PT } & \multicolumn{2}{|c|}{ Modelo PST } \\
\hline & Estimativa & EP & Estimativa & EP & Estimativa & EP & Estimativa & EP \\
\hline$\xi$ & 14.7835 & 0.3615 & 4.7469 & 0.6892 & 8.4027 & 0.7923 & 11.5509 & 0.1337 \\
\hline$\eta$ & 11.0045 & 0.3975 & 14.1532 & 0.7237 & 11.8146 & 0.4707 & 22.6885 & 0.0792 \\
\hline$\lambda$ & - & - & 1.5673 & 0.1838 & - & - & 5.2347 & 0.2870 \\
\hline$\alpha$ & - & - & - & - & & 0.1147 & & 0.0347 \\
\hline D & 3.4156 & 0.3601 & 3.4075 & 0.3454 & 2.7473 & 0.2566 & 12.8734 & 2.9729 \\
\hline
\end{tabular}

t-potência (PT) e t-assimétrico-potência (PST). Para comparar os modelos ajustados, usamos o Critério de Informação de Akaike (AIC), (Akaike, 1974), em que $A I C=-2 \ell(\widehat{\boldsymbol{\theta}} \mid \mathbf{y})+2 k$; Critério de informação Bayesiano (BIC), (Schwarz, 1978), em que $B I C=-2 \ell(\widehat{\boldsymbol{\theta}} \mid \mathbf{y})+\log (n) k$, e o AIC consistente, (CIAC) (Bozdogan, 1987), em que CAIC $=-2 \ell(\widehat{\boldsymbol{\theta}} \mid \mathbf{y})+k(1+\log (n))$. Em todos os critérios temos que $k$ é o número de parâmetros no modelo ajustado, e $n$ é o tamanho da amostra.

A Tabela 3.3 apresenta as estimativas de máxima verossimilhança e os erros padrões (EP) para os modelos T, ST, PT e PST. Note-se que os valores dos erros padrão das estimativas de $\xi$ e $\eta$ no modelo PST são menores que os correspondentes erros padrões dos respectivos parâmetros para os modelos T, ST e PT. A Tabela 3.4 mostra os critérios de seleção considerados, além do valor da log-verossimilhança obtida para cada modelo considerado. As estimativas dos parâmetros foram obtidas pela maximização da função de verossimilhança usando o método de optimização numérico BFGS na função "optim" do pacote estatístio R (R Development Core Team, 2017).

De acordo com os critérios AIC, BIC e CAIC, o modelo PST parece proporcionar um melhor ajuste aos dados de vulcões que os modelos T, ST e PT, apoiando a afirmação de afastamento da simetria da variável altitude dos vulcões $(Y)$.

A Figura 3.3 mostra os gráficos qqplot para os modelos ajustados. Dessa figura, pode-se ver claramente que o modelo PST se ajusta melhor aos dados do que os modelos T, ST, PT. Além disso, o teste de Kolmogorov-Smirnov produz a estatística de teste $D=0,0027$ com $p$-value $=0,6379$, indicando que o modelo PST é capaz de ajustar os dados sob estudo.

Tabela 3.4: Critérios de seleção dos modelos ajustados aos dados de vulcões.

\begin{tabular}{lcccc}
\hline Critério & Model T & Modelo ST & Modelo PT & Modelo PST \\
\hline$\ell(\boldsymbol{\theta})$ & -6273.35 & -6219.25 & -6228.77 & -6205.94 \\
AIC & 12552.70 & 12446.49 & 12465.53 & 12421.87 \\
BIC & 12568.68 & 12467.79 & 12486.53 & 12448.50 \\
CAIC & 12571.68 & 12471.79 & 12490.83 & 12453.50 \\
\hline
\end{tabular}




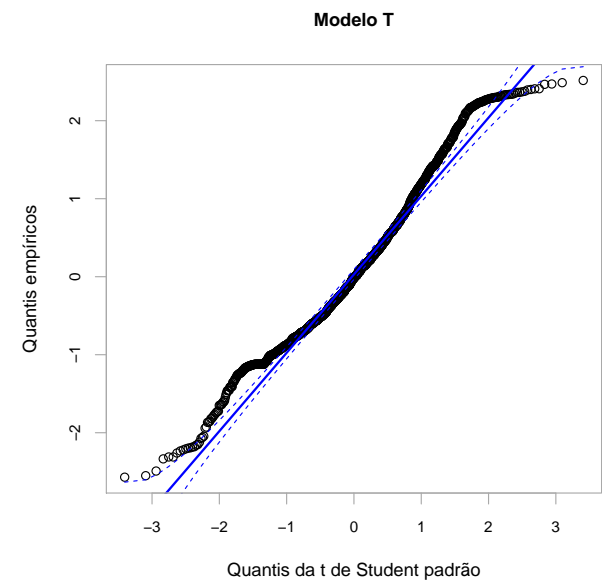

(a)

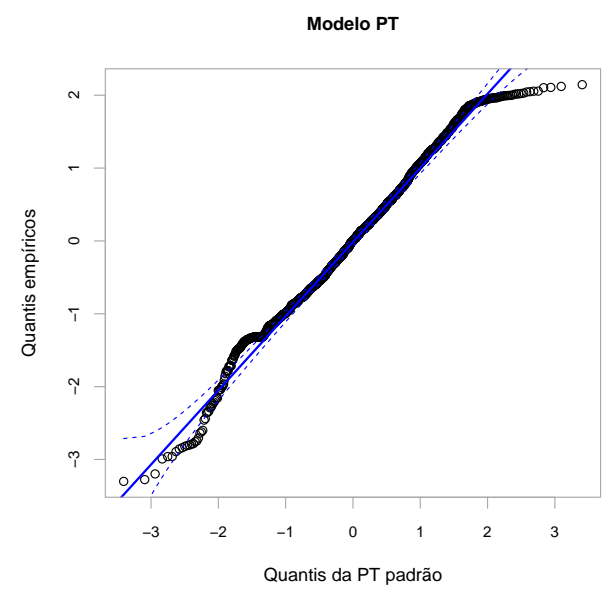

(c)

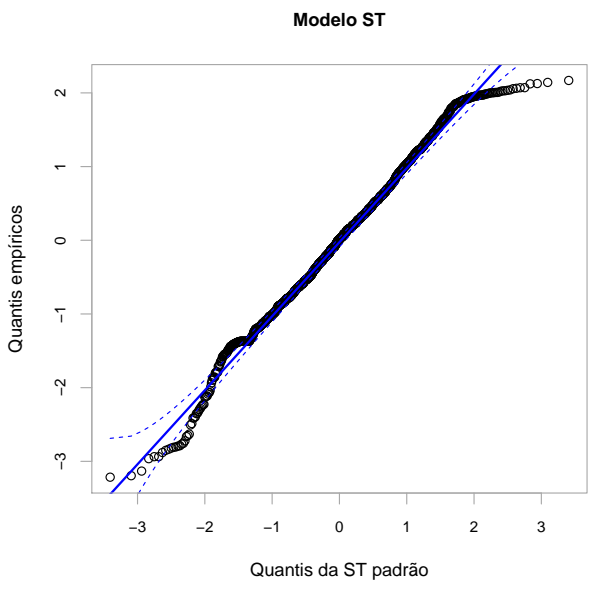

(b)

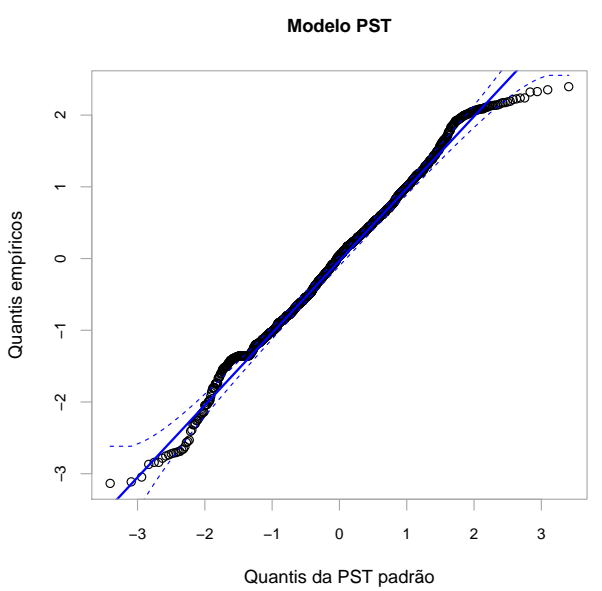

(d)

Figura 3.3: Envelopes dos resíduos quantis aleatorizados para (a) Modelo T, (b) Modelo ST, (c) Model PT, (d) Modelo PST.

A Figura 3.4 (a) mostra o histograma da variável $Y$ (altitude), enquanto que, a Figura 3.4 (b) apresenta a fda empírica (linha sólida) junto com a fda do modelo PST ajustado (linha pontilhada).

Consideramos agora testar a hipótese de nenhuma diferença entre as distribuições $t$ de Student e PST para os dados de altitude dos vulcões, o que corresponde ao teste da hipótese

$$
H_{0}:(\lambda, \alpha)=(0,1) \quad \text { contra } \quad H_{1}:(\lambda, \alpha) \neq(0,1),
$$

usando a estatística de razão de verossimilhanças

$$
\Lambda=\frac{L_{T}(\widehat{\boldsymbol{\theta}})}{L_{P S T}(\widehat{\boldsymbol{\theta}})}
$$




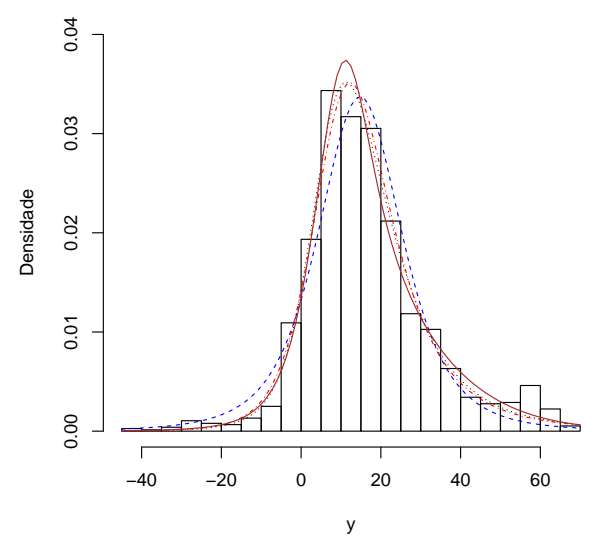

(a)

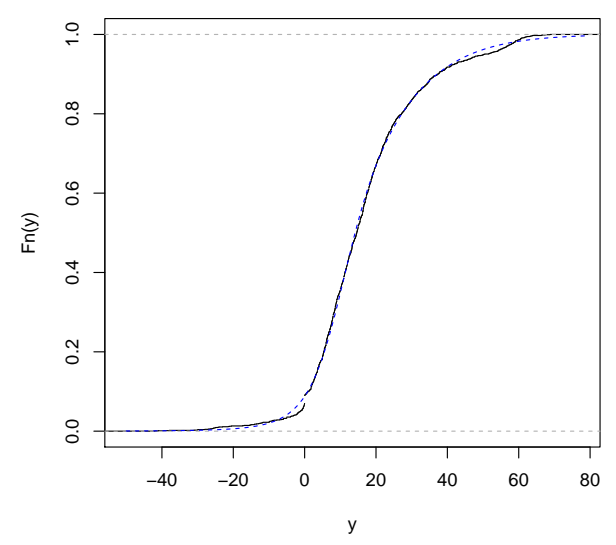

(b)

Figura 3.4: (a) Gráfico dos modelos ajustados aos dados de vulcões. PST (linha sólida), T (linha tracejada), PS (linha pontilhada) e ST (linha pontilhada-tracejada). (b) fda empírica de $Y$ e fda de modelo PST ajustado.

que produz o valor

$$
-2 \log (\Lambda)=134.823
$$

sendo maior que o valor crítico $5 \%$ chi-quadrado, $\chi_{5,95 \%}^{2}=5,99$. Portanto, o modelo PST se ajusta melhor aos dados do que o modelo $t$ de Student. O modelo PST também é comparado aos modelos ST e PT testando as hipóteses

$$
H_{01}: \lambda=0 \quad \text { contra } \quad H_{11}: \lambda \neq 0, \quad \text { e } \quad H_{02}: \alpha=1 \quad \text { contra } \quad H_{12}: \alpha \neq 1,
$$

respectivamente, usando a razão de verossimilhanças

$$
\Lambda_{1}=\frac{L_{S T}(\widehat{\boldsymbol{\theta}})}{L_{P S T}(\widehat{\boldsymbol{\theta}})} \quad \text { e } \quad \Lambda_{2}=\frac{L_{P T}(\widehat{\boldsymbol{\theta}})}{L_{P S T}(\widehat{\boldsymbol{\theta}})} .
$$

Após avaliações numéricas, obtemos

$$
-2 \log \left(\Lambda_{1}\right)=26.620 \quad \text { e } \quad-2 \log \left(\Lambda_{2}\right)=45.660,
$$

que são maiores que o valor $\chi_{1,95 \%}^{2}=3.84$. Note-se que o modelo PST proporciona melhor ajuste ao conjunto de dados analisado.

\subsection{Modelo t-Potência para Dados Censurados e Truncados}

Nesta seção introduzimos agora a extensão do modelo PT às situações em que os dados apresentam censura ou truncamento num valor da variável resposta.

\subsubsection{O Modelo t-Potência para Dados Censurados}

Inicialmente, suponha que a variável aleatória $Y$ tem uma distribuição PT de parâmetros $\xi$, $\eta, \alpha$ e $\nu$; e consideremos uma amostra $Y_{1}, \ldots, Y_{n}$, de tamanho $n$ de $Y$, em que somente relatamos 
aqueles valores $Y_{i}$ maiores que uma constante $k_{i}$; e que para valores $Y_{i} \leq k_{i}$ somente o valor $k_{i}$ é relatado. Os valores observados $Y_{i}^{o}$ podem ser escritos da forma

$$
Y_{i}^{o}= \begin{cases}k_{i}, & \text { se } Y_{i} \leq k_{i} \\ Y_{i}, & \text { se } Y_{i}>k_{i}\end{cases}
$$

para $i=1, \ldots, n$. A amostra resultante é dita ser uma amostra PT censurada. Por conveniência, escolhemos trabalhar com o caso de censura à esquerda, mas os resultados podem ser estendíveis para outros tipos de censura.

Para observações $Y_{i}^{o}=k_{i}$, temos que $P\left(Y_{i}^{o}=k_{i}\right)=P\left(Y_{i} \leq k_{i}\right)=\left\{T\left(\frac{k_{i}-\xi}{\eta} \mid \nu\right)\right\}^{\alpha}$, e para observações $Y_{i}^{o}=Y_{i}$ a distribuição de $Y_{i}^{o}$ é a mesma de $Y_{i}$, isto é, $Y_{i}^{o} \sim \mathrm{PT}(\xi, \eta, \alpha, \nu)$.

Temos interesse no vetor de parâmetros $\boldsymbol{\theta}=(\xi, \eta, \alpha, \nu)^{\top}$. A inferência estatística considerada para estimar $\boldsymbol{\theta}$ é baseada no método da máxima verossimilhança Assim, procedendo como Arellano-Valle et al. (2012), usamos a reparametrização proposta por Olsen (1978) e fazemos $\gamma=\sigma \xi$, e $\sigma=1 / \eta$, então segue que a função de log-verossimilhança para o vetor $\varphi=(\gamma, \sigma, \alpha, \nu)^{\top}$, dada uma amostra observada $\mathbf{Y}^{o}$, é dada por

$$
\begin{aligned}
\ell\left(\boldsymbol{\varphi} ; \mathbf{Y}^{o}\right)=\alpha & \sum_{i=1}^{n}\left(1-d_{i}\right) \log \left\{T\left(c_{i} \mid \nu\right)\right\} \\
& +\sum_{i=1}^{n} d_{i}\left\{\log \alpha+\log \sigma+\log t\left(z_{i} \mid \nu\right)+(\alpha-1) \log T\left(z_{i} \mid \nu\right)\right\}
\end{aligned}
$$

em que $c_{i}=\sigma k_{i}-\gamma, z_{i}=\sigma y_{i}-\gamma$ e $d_{i}$ é uma variável indicadora com $d_{i}=1$ se $Y_{i}^{o}=Y_{i}$; e $d_{i}=0$ se $Y_{i}^{o}=k_{i}$. Os componentes da função escore $\boldsymbol{U}(\boldsymbol{\varphi})$ são obtidos derivando parcialmente $\ell\left(\boldsymbol{\varphi} ; \mathbf{Y}^{o}\right)$ em (3.24) com relação às componentes $\gamma, \sigma, \alpha$ e $\nu$, e ficam dados por

$$
\begin{aligned}
U_{\gamma}(\boldsymbol{\varphi})= & \alpha \sum_{i=1}^{n}\left(1-d_{i}\right) r\left(c_{i} \mid \nu\right) \\
& +\sum_{i=1}^{n} d_{i}\left\{\left(\frac{\nu+1}{\nu}\right)\left(1+\frac{z_{i}^{2}}{\nu}\right)^{-1} z_{i}-(\alpha-1) r\left(z_{i} \mid \nu\right)\right\} \\
U_{\sigma}(\boldsymbol{\varphi})=\alpha & \sum_{i=1}^{n}\left(1-d_{i}\right) r\left(c_{i} \mid \nu\right) k_{i} \\
& +\sum_{i=1}^{n} d_{i}\left\{\frac{1}{\sigma}-\left(\frac{\nu+1}{\nu}\right)\left(1+\frac{z_{i}^{2}}{\nu}\right)^{-1} z_{i} y_{i}+(\alpha-1) r\left(z_{i} \mid \nu\right) y_{i}\right\} \\
U_{\nu}(\boldsymbol{\varphi})=\frac{c^{2}}{2} \sum_{i=1}^{n}\left(1-d_{i}\right)\left\{\psi\left(\frac{\nu+1}{2}\right)-\psi\left(\frac{\nu}{2}\right)-b_{01}\left(c_{i} \mid \nu\right)-\frac{c_{i} r\left(c_{i} \mid \nu\right)}{\nu}\right\} & \\
& +\frac{1}{2} \sum_{i=1}^{n} d_{i}\left\{\psi\left(\frac{\nu+1}{2}\right)-\psi\left(\frac{\nu}{2}\right)-\frac{1}{\nu}\right. \\
& \left.-\log \left(1+\frac{z_{i}^{2}}{\nu}\right)+\left(\frac{\nu+1}{\nu}\right)\left(1+\frac{z_{i}^{2}}{\nu}\right)^{-1} \frac{z_{i}^{2}}{\nu}\right\} \\
& +\frac{\alpha-1}{2} \sum_{i=1}^{n} d_{i}\left\{\psi\left(\frac{\nu+1}{2}\right)-\psi\left(\frac{\nu}{2}\right)-b_{01}\left(z_{i} \mid \nu\right)-\frac{z_{i} r\left(z_{i} \mid \nu\right)}{\nu}\right\}
\end{aligned}
$$




$$
U_{\alpha}(\boldsymbol{\varphi})=\sum_{i=1}^{n}\left(1-d_{i}\right) \log \left\{T\left(c_{i} \mid \nu\right)\right\}+\sum_{i=1}^{n} d_{i}\left\{\frac{1}{\alpha}+\log T\left(z_{i} \mid \nu\right)\right\}
$$

em que $r(x \mid \nu)=t(x \mid \nu) / T(x \mid \nu), \psi(x)=\frac{d}{d x} \log \Gamma(x)$ é a função digama e $b_{m n}\left(c_{m} \mid \nu+m\right)$ é o momento truncado definido por

$$
b_{m n}\left(c_{m} \mid \nu+m\right)=\int_{-\infty}^{c_{m}} s^{m}\left\{\log \left(1+\frac{s^{2}}{\nu+m}\right)\right\}^{n} \frac{t(s \mid \nu+m)}{T\left(c_{m} \mid \nu+m\right)} d s,
$$

com $c_{m}=\sqrt{\frac{\nu+m}{\nu}} c$ e $c_{0}=c$. Os momentos $b_{m n}$ em (3.29) são obtidos por integração numérica, por exemplo a função integrate no pacote estatístico $R$.

Os elementos da matriz de informação observada, denotados por $j_{\gamma \gamma}, j_{\gamma \sigma}, \ldots, j_{\alpha \alpha}$; são obtidos multiplicando por -1 a segunda derivada parcial da função de log-verossimilhança com respeito a cada um dos parâmetros, isto é, $j_{\boldsymbol{\varphi}_{i} \boldsymbol{\varphi}_{k}}=-\partial^{2} \ell\left(\boldsymbol{\varphi} ; \mathbf{Y}^{o}\right) / \partial \boldsymbol{\varphi}_{i} \partial \boldsymbol{\varphi}_{k}$. Estes elementos $j_{\boldsymbol{\varphi}_{i} \boldsymbol{\varphi}_{k}}$ são apresentados no Apêndice B.2.

Para recuperar a matriz de informação observada $\boldsymbol{J}(\boldsymbol{\theta})$ da parametrização original $\boldsymbol{\theta}=$ $(\xi, \eta, \alpha, \nu)^{\top}$, temos que $\boldsymbol{J}(\boldsymbol{\theta})=(\partial \boldsymbol{\varphi} / \partial \boldsymbol{\theta})^{\top} \boldsymbol{J}(\boldsymbol{\varphi})(\partial \boldsymbol{\varphi} / \partial \boldsymbol{\theta})$, em que a matriz Jacobiana é

$$
\frac{\partial \boldsymbol{\varphi}}{\partial \boldsymbol{\theta}}=\left(\begin{array}{cccc}
\frac{1}{\eta} & -\frac{1}{\eta^{2}} & 0 & 0 \\
0 & -\frac{1}{\eta^{2}} & 0 & 0 \\
0 & 0 & 1 & 0 \\
0 & 0 & 0 & 1
\end{array}\right)
$$

As estimativas de máxima verossimilhança dos parâmetros $\xi, \eta, \alpha$, e $\nu$ no modelo PT censurado são obtidas usando algoritmos iterativos baseados nas funções escore (3.25) - (3.28) e aplicando a transformação inversa $\xi=\gamma / \sigma$ e $\eta=1 / \sigma$. As estimativas das variâncias dos estimadores podem ser obtidas avaliando a inversa da matriz de informação observada $\boldsymbol{J}^{-1}(\boldsymbol{\varphi})$ nos estimadores de máxima verossimilhança $\widehat{\varphi}=(\widehat{\gamma}, \widehat{\sigma}, \widehat{\alpha}, \widehat{\nu})^{\top}$ e pelo uso do resultado acima.

\subsubsection{O Modelo $t$-Potência para Dados Truncados}

Vamos considerar agora que antes da amostra ser selecionada, a distribuição de $Y$ é truncada no valor $k$, de modo que somente podemos escolher observações tais que $Y \leq k$. Segue que a variavel aleatória $Y$ tem uma distribuição PT truncada e pela Definição 5, tem-se que a fdp de $Y$ é dada por

$$
f_{T P T}(y \mid \xi, \eta, \alpha ; y \leq k)=\frac{\alpha}{\eta} t\left(\frac{y-\xi}{\eta} \mid \nu\right)\left[T\left(\frac{y-\xi}{\eta} \mid \nu\right)\right]^{\alpha-1}\left[T\left(\frac{k-\xi}{\eta} \mid \nu\right)\right]^{-\alpha}
$$

para $-\infty<y \leq k$. Novamente, usamos a reparametrização proposta por Olsen (1978), em que $\gamma=\sigma \xi$ e $\sigma=1 / \eta$, e temos que a função de $\log$-verossimilhança para o vetor $\boldsymbol{\varphi}=(\gamma, \sigma, \alpha, \nu)^{\top}$, dada uma amostra $\mathbf{Y}$ é dada por

$$
\begin{aligned}
\ell(\boldsymbol{\varphi} ; \mathbf{Y})=- & n_{1} \alpha \log \{T(c \mid \nu)\} \\
& +\sum_{y_{i} \leq k}^{n}\left\{\log \alpha+\log \sigma+\log t\left(z_{i} \mid \nu\right)+(\alpha-1) \log T\left(z_{i} \mid \nu\right)\right\}
\end{aligned}
$$

em que $c=\sigma k-\gamma, z_{i}=\sigma y_{i}-\gamma$, e $n_{1}$ é o número de observações na amostra tais que $-\infty<y \leq k$. Derivando parcialmente a função de log-verossimilhança (3.32) com respecto aos componentes 
do vetor $\varphi$ obtemos os seguintes elementos da função escore

$$
\begin{aligned}
U_{\boldsymbol{\gamma}}(\boldsymbol{\varphi})= & n_{1} \alpha r(c \mid \nu)+\sum_{y_{i} \leq k}\left\{\left(\frac{\nu+1}{\nu}\right)\left(1+\frac{z_{i}^{2}}{\nu}\right)^{-1} z_{i}-(\alpha-1) r\left(z_{i} \mid \nu\right)\right\} \\
U_{\sigma}(\boldsymbol{\varphi})=- & n_{1} \alpha r(c \mid \nu) k+\sum_{y_{i} \leq k}\left\{\frac{1}{\sigma}-\left(\frac{\nu+1}{\nu}\right)\left(1+\frac{z_{i}^{2}}{\nu}\right)^{-1} z_{i}+(\alpha-1) r\left(z_{i} \mid \nu\right)\right\} \\
U_{\nu}(\boldsymbol{\varphi})=- & \frac{n_{1} \alpha}{2}\left\{\psi\left(\frac{\nu+1}{2}\right)-\psi\left(\frac{\nu}{2}\right)-b_{01}(c \mid \nu)-\frac{c r(c \mid \nu)}{\nu}\right\} \\
& +\frac{1}{2} \sum_{y_{i} \leq k}\left\{\psi\left(\frac{\nu+1}{2}\right)-\psi\left(\frac{\nu}{2}\right)-\frac{1}{\nu}\right. \\
& \left.-\log \left(1+\frac{z_{i}^{2}}{\nu}\right)+\left(\frac{\nu+1}{\nu}\right)\left(1+\frac{z_{i}^{2}}{\nu}\right)^{-1} \frac{z_{i}^{2}}{\nu}\right\} \\
& +\frac{\alpha-1}{2} \sum_{y_{i} \leq k}\left\{\psi\left(\frac{\nu+1}{2}\right)-\psi\left(\frac{\nu}{2}\right)-b_{01}\left(z_{i} \mid \nu\right)-\frac{z_{i} r\left(z_{i} \mid \nu\right)}{\nu}\right\} \\
U_{\alpha}(\boldsymbol{\varphi})=- & n_{1} \log \{T(c \mid \nu)\}+\sum_{y_{i} \leq k}\left\{\frac{1}{\alpha}+\log T\left(z_{i} \mid \nu\right)\right\}
\end{aligned}
$$

em que $r(x \mid \nu), \psi(x)$ e $b_{m n}\left(c_{m} \mid \nu+m\right)$ são dados como no modelo PT censurado.

Para obter as estimativas de máxima verossimilhança dos parâmetros $\xi, \eta, \alpha$ e $\nu$ no modelo PT truncado, procedemos como no modelo PT censurado, e usamos métodos iterativos baseados no algoritmo de Newton-Rapshon usando as funções escore (3.33) - (3.36). De novo, para para recuperar a matriz de informação observada $\boldsymbol{J}(\boldsymbol{\theta})$ da parametrização original $\boldsymbol{\theta}=(\xi, \eta, \alpha, \nu)^{\top}$, usamos $\boldsymbol{J}(\boldsymbol{\theta})=(\partial \boldsymbol{\varphi} / \partial \boldsymbol{\theta})^{\top} \boldsymbol{J}(\boldsymbol{\varphi})(\partial \boldsymbol{\varphi} / \partial \boldsymbol{\theta})$, em que $\frac{\partial \boldsymbol{\varphi}}{\partial \boldsymbol{\theta}}$ é dado em (3.30). No Apêndice B.3 são apresentadosos elementos da matriz de informação observada $\boldsymbol{J}(\boldsymbol{\varphi})$, denotados por $j_{\gamma \gamma}, j_{\gamma \sigma}, \ldots, j_{\alpha \alpha}$.

\subsection{O Modelo de Regressão Linear t-Potência Censurado}

Motivados pelas grandes virtudes da distribuição PT para ajustar dados com alto grau de assimetria e curtose maior ou menor do que o modelo $t$ de Student usual, introduzimos nesta seção o modelo de regressão linear $t$-Potência Censurado, que é denotado por modelo PT-CR, (Power-t Censored Regression Model).

O modelo PT-CR resulta da consideração da variável aleatória observada $Y_{i}^{o}=D_{i} Y_{i}$, em que $D_{i}=I\left(Y_{i}>0\right)$ e $Y_{i}=\mathbf{x}_{i}^{\top} \boldsymbol{\beta}+\epsilon_{i}, \operatorname{com} \epsilon_{i} \stackrel{\text { iid }}{\sim} \mathrm{PT}(0, \eta, \alpha, \nu), i=1, \ldots, n$; isto é,

$$
Y_{i}^{o}= \begin{cases}\mathbf{x}_{i}^{\top} \boldsymbol{\beta}+\epsilon_{i}, & \text { if } Y_{i}>0 \\ 0, & \text { if } Y_{i} \leq 0\end{cases}
$$

em que $\boldsymbol{\beta}$ é um vetor de dimensão $p$ de parâmetros desconhecidos, $\mathbf{x}_{i}=\left(x_{i 1}, \ldots, x_{i p}\right)^{\top}, i=$ $1, \ldots, n$, são vetores de covariáveis conhecidas, e $\epsilon_{i}, i=1, \ldots, n$, são variáveis aleatórias independentes com distribuição PT, com parâmetro de locação 0, parâmetro de escala $\eta$, parâmetro de forma $\alpha$, e graus de liberdade $\nu$. A suposição anterior é equivalente a considerar que as variáveis aleatórias não observadas $Y_{1}, \ldots, Y_{n}$ são independentes, com $Y_{i} \sim \mathrm{PT}\left(\mathbf{x}_{i}^{\top} \boldsymbol{\beta}, \eta, \alpha, \nu\right)$, isto é, com fdp dada por

$$
f\left(y_{i} \mid \boldsymbol{\theta}\right)=\frac{\alpha}{\eta} t\left(\frac{y_{i}-\mathbf{x}_{i}^{\top} \boldsymbol{\beta}}{\eta} \mid \nu\right)\left[T\left(\frac{y_{i}-\mathbf{x}_{i}^{\top} \boldsymbol{\beta}}{\eta} \mid \nu\right)\right]^{\alpha-1}
$$


$i=1, \ldots, n$, em que $\boldsymbol{\theta}=\left(\boldsymbol{\beta}^{\top}, \eta, \alpha, \nu\right)^{\top}$. Para observacões $Y_{i}^{o}$ iguais a zero, a contribuição à função de verossimilhança é dada por

$$
\mathrm{P}\left(Y_{i}^{o}=0\right)=\left\{T\left(-\frac{\mathbf{x}_{i}^{\top} \boldsymbol{\beta}}{\eta} \mid \nu\right)\right\}^{\alpha}
$$

e para observações $Y_{i}^{o}$ maiores que zero, tem-se que $Y_{i}^{o} \sim \mathrm{PT}\left(\mathbf{x}_{i}^{\top} \boldsymbol{\beta}, \eta, \alpha, \nu\right)$. Portanto, a função de verossimilhança do modelo PT-CR baseada em uma amostra observada $\mathbf{Y}^{o}$ é dada por

$$
\begin{aligned}
L\left(\boldsymbol{\theta} ; \mathbf{Y}^{o}\right)=\prod_{i=1}^{n}\{ & \left.T\left(-\frac{\mathbf{x}_{i}^{\top} \boldsymbol{\beta}}{\eta} \mid \nu\right)\right\}^{\alpha\left(1-d_{i}\right)}\left\{\frac{\alpha}{\eta} t\left(\frac{y_{i}-\mathbf{x}_{i}^{\top} \boldsymbol{\beta}}{\eta} \mid \nu\right)\right. \\
& \left.\times\left[T\left(\frac{y_{i}-\mathbf{x}_{i}^{\top} \boldsymbol{\beta}}{\eta} \mid \nu\right)\right]^{\alpha-1}\right\}^{d_{i}}
\end{aligned}
$$

em que

$$
d_{i}= \begin{cases}1, & \text { if } Y_{i}^{o}>0 \\ 0, & \text { if } Y_{i}^{o}=0,\end{cases}
$$

O modelo (3.37) é equivalente à situação mais geral em que o valor da censura associado à observação $i$ é substituído pelo valor $k_{i}$ (um valor conhecido), isto é,

$$
Y_{i}^{o}= \begin{cases}\mathbf{x}_{i}^{\top} \boldsymbol{\beta}+\epsilon_{i}, & \text { if } Y_{i}>k_{i}, \\ k_{i}, & \text { if } Y_{i} \leq k_{i},\end{cases}
$$

$i=1, \ldots, n$. Note-se que, fazendo $Y_{i}^{o *}=Y_{i}^{o}-k_{i}, \mathbf{x}_{i}^{*}=\left(\mathbf{x}_{i}^{\top}, k_{i}\right)^{\top}$ e $\boldsymbol{\beta}^{*}=\left(\boldsymbol{\beta}^{\top},-1\right)^{\top}$, tem-se o modelo previo em (3.37), assim, os resultados da inferência baseados no método da máxima verossimilhança podem ser usados para ajustar o modelo mais geral em (3.39).

Pode-se observar que o modelo (3.38) se reduz ao modelo de regressão $t$ de Student censurado (Arellano-Valle et al., 2012) quando $\alpha=1$; e converge para o modelo Tobit normal-potência quando $\nu \rightarrow \infty$, (Martínez-Flórez et al., 2013b). Finalmente, o modelo Tobit usual (Tobin, 1958) é obtido quando $\alpha=1$ e $\nu \rightarrow \infty$.

\subsubsection{Momentos}

A média e a variância para a $i$-ésima resposta observada no modelo PT-CR é obtida desde que $Y_{i}^{o}=D_{i} Y_{i}$, em que $Y_{i}=\mu_{i}+\eta Z_{i}$, com $\mu_{i}=\mathbf{x}_{i}^{\top} \boldsymbol{\beta}$ e $Z_{i} \stackrel{\text { ind }}{\sim} \operatorname{PT}(0,1, \alpha, \nu), i=1, \ldots, n$. Segue que

$$
\begin{aligned}
\mathrm{E}\left[Y_{i}^{o}\right]= & \mathrm{E}\left[Y_{i} \mid Y_{i}>0\right] \mathrm{P}\left(Y_{i}>0\right) \\
= & \left(\mu_{i}+\eta \mathrm{E}\left[Z_{i} \mid Z_{i}+c_{i}>0\right]\right)\left(1-\left\{T\left(-c_{i} \mid \nu\right)\right\}^{\alpha}\right) \\
\operatorname{Var}\left[Y_{i}^{o}\right]= & \mathrm{E}\left[Y_{i}^{2} \mid Y_{i}>0\right] \mathrm{P}\left(Y_{i}>0\right)-\left(\mathrm{E}\left[Y_{i}^{o}\right]\right)^{2} \\
= & \left(\mu_{i}^{2}+2 \eta \mu_{i} \mathrm{E}\left[Z_{i} \mid Z_{i}+c_{i}>0\right]\right)\left(\left\{T\left(-c_{i} \mid \nu\right)\right\}^{\alpha}\right) \\
& \quad \times\left(1-\left\{T\left(-c_{i} \mid \nu\right)\right\}^{\alpha}\right) \\
& +\eta^{2}\left(\mathrm{E}\left[Z_{i}^{2} \mid Z_{i}+c_{i}>0\right]\left(1-\left\{T\left(-c_{i} \mid \nu\right)\right\}^{\alpha}\right)\right. \\
& \left.-\left(\mathrm{E}\left[Z_{i} \mid Z_{i}+c_{i}>0\right]\right)^{2}\left(1-\left\{T\left(-c_{i} \mid \nu\right)\right\}^{\alpha}\right)^{2}\right)
\end{aligned}
$$


em que $c_{i}=\mu_{i} / \eta$, e pela Proposição 3 , tem-se que

$$
\begin{aligned}
\mathrm{E}\left[Y_{i}^{o}\right]=\eta & \left\{c_{i}+m_{1}\left(c_{i} \mid \nu\right)\right\}\left(1-\left\{T\left(-c_{i} \mid \nu\right)\right\}^{\alpha}\right) \\
\operatorname{Var}\left[Y_{i}^{o}\right]=\eta^{2} & \left\{c_{i}^{2}+2 c_{i} m_{1}\left(c_{i} \mid \nu\right)\right\}\left(\left\{T\left(-c_{i} \mid \nu\right)\right\}^{\alpha}\right) \\
& \times\left(1-\left\{T\left(-c_{i} \mid \nu\right)\right\}^{\alpha}\right) \\
& +\eta^{2}\left\{m_{2}\left(c_{i} \mid \nu\right)-\left[m_{1}\left(c_{i} \mid \nu\right)\right]^{2}\left(1-\left\{T\left(-c_{i} \mid \nu\right)\right\}^{\alpha}\right)\right\} \\
& \times\left(1-\left\{T\left(-c_{i} \mid \nu\right)\right\}^{\alpha}\right)
\end{aligned}
$$

em que

$$
m_{r}(c \mid \nu)=\frac{1}{1-\{T(-c \mid \nu)\}^{\alpha}} \int_{T(-c \mid \nu)}^{1}\left[T^{-1}(u \mid \nu)\right]^{r} \alpha u^{\alpha-1} d u .
$$

com $T^{-1}(\cdot \mid \nu)$ a inversa da fda $T(\cdot \mid \nu)$. Note que $m_{r}(c \mid \nu)$ é o momento $\mathrm{E}\left[\left(T^{-1}(U \mid \nu)\right)^{r}\right]$ de uma variável aleatória beta de parâmetros $\alpha$ e 1, truncada em $A=[T(-c \mid \nu), 1]$.

É importante ressaltar que $\mathrm{E}\left[Y_{i}^{o}\right]$ e $\operatorname{Var}\left[Y_{i}^{o}\right]$ são definidos para todo $\nu>0$, e quando $\nu \rightarrow \infty$ segue que (3.40) e (3.41) convergem para a média e a variância do modelo Tobit normal-potência (Martínez-Flórez et al., 2013b), respectivamente, isto é, quando $\nu \rightarrow \infty$

$$
\begin{aligned}
\mathrm{E}\left[Y_{i}^{o}\right] \longrightarrow & \eta\left\{c_{i}+m_{1}\left(c_{i}\right)\right\}\left(1-\left\{\Phi\left(-c_{i}\right)\right\}^{\alpha}\right) \\
\operatorname{Var}\left[Y_{i}^{o}\right] \longrightarrow & \eta^{2}\left\{c_{i}^{2}+2 c_{i} m_{1}\left(c_{i}\right)\right\}\left(\left\{\Phi\left(-c_{i}\right)\right\}^{\alpha}\right)\left(1-\left\{\Phi\left(-c_{i}\right)\right\}^{\alpha}\right) \\
& +\eta^{2}\left\{m_{2}\left(c_{i}\right)-\left[m_{1}\left(c_{i}\right)\right]^{2}\left(1-\left\{\Phi\left(-c_{i}\right)\right\}^{\alpha}\right)\right\}\left(1-\left\{\Phi\left(-c_{i}\right)\right\}^{\alpha}\right)
\end{aligned}
$$

em que

$$
m_{r}(c)=\frac{1}{1-\{\Phi(-c)\}^{\alpha}} \int_{\Phi(-c)}^{1}\left[\Phi^{-1}(u)\right]^{r} \alpha u^{\alpha-1} d u .
$$

com $\Phi(\cdot)$ a fda da distribuição normal padronizada, e $\Phi^{-1}(\cdot)$ a função inversa de $\Phi(\cdot)$. Vale a pena ressaltar também que, se $\alpha=1$ e $\nu \rightarrow \infty$, temos $m_{1}\left(c_{i} \mid \nu\right) \longrightarrow \phi\left(c_{i}\right) / \Phi\left(c_{i}\right)$ e $m_{2}\left(c_{i} \mid \nu\right) \longrightarrow$ $\left(\Phi\left(c_{i}\right)-c_{i} \phi\left(c_{i}\right)\right) / \Phi\left(c_{i}\right)$, portanto

$$
\begin{aligned}
& \mathrm{E}\left[Y_{i}^{o}\right] \longrightarrow \eta\left\{c_{i} \Phi\left(c_{i}\right)+\phi\left(c_{i}\right)\right\} \\
& \operatorname{Var}\left[Y_{i}^{o}\right] \longrightarrow \eta^{2}\left\{c_{i}^{2} \Phi\left(c_{i}\right)+2 c_{i} \phi\left(c_{i}\right)\right\}\left(1-\Phi\left(c_{i}\right)\right) \\
& +\eta^{2}\left\{\Phi\left(c_{i}\right)-\phi\left(c_{i}\right)\left[c_{i}+\phi\left(c_{i}\right)\right]\right\}
\end{aligned}
$$

os quais são respectivamente, a média e a variância do modelo Tobit usual.

\subsubsection{Estimação}

No modelo PT-CR o vetor de parâmetros de interesse é $\boldsymbol{\theta}=(\boldsymbol{\beta}, \eta, \alpha, \nu)^{\top}$, e para fazer a inferência consideramos o método da máxima versossimilhança e usamos novamente a reparametrização de Olsen (1978), em que $\boldsymbol{\gamma}=\sigma \boldsymbol{\beta}$, e $\sigma=1 / \eta$. Definindo

$$
d_{i}= \begin{cases}1, & \text { if } Y_{i}^{o}>k_{i}, \\ 0, & \text { if } Y_{i}^{o}=k_{i},\end{cases}
$$


se segue que a função de log-verossimilhança para $\varphi=\left(\gamma^{\top}, \sigma, \alpha, \nu\right)^{\top}$ obtida de (3.38) sob a nova parametrização é dada por

$$
\begin{aligned}
\ell\left(\boldsymbol{\varphi} ; \mathbf{Y}^{o}\right)= & \alpha \sum_{i=1}^{n}\left(1-d_{i}\right) \log \left\{1-T\left(c_{i} \mid \nu\right)\right\} \\
& +\sum_{i=1}^{n} d_{i}\left\{\log \alpha+\log \sigma+\log t\left(z_{i} \mid \nu\right)+(\alpha-1) \log T\left(z_{i} \mid \nu\right)\right\}
\end{aligned}
$$

em que $z_{i}=\sigma y_{i}-c_{i}$, com $c_{i}=\mathbf{x}_{i}^{\top} \boldsymbol{\gamma}$. Os componentes da função escore $\boldsymbol{U}(\boldsymbol{\varphi})$ são obtidos derivando $\ell\left(\boldsymbol{\varphi} ; \mathbf{Y}^{o}\right)$ parcialmente com relação aos componentes $\gamma, \eta, \alpha$ e $\nu$. Depois de algumas manipulações algébricas obtemos as seguintes componentes da função escore

$$
\begin{aligned}
U_{\boldsymbol{\gamma}}(\boldsymbol{\varphi})= & -\alpha \sum_{i=1}^{n}\left(1-d_{i}\right) r\left(-c_{i} \mid \nu\right) \mathbf{x}_{i} \\
& +\sum_{i=1}^{n} d_{i}\left\{\left(\frac{\nu+1}{\nu}\right)\left(1+\frac{z_{i}^{2}}{\nu}\right)^{-1} z_{i}-(\alpha-1) r\left(z_{i} \mid \nu\right)\right\} \mathbf{x}_{i} \\
U_{\sigma}(\boldsymbol{\varphi})= & \sum_{i=1}^{n} d_{i}\left\{\frac{1}{\sigma}-\left(\frac{\nu+1}{\nu}\right)\left(1+\frac{z_{i}^{2}}{\nu}\right)^{-1} z_{i} y_{i}+(\alpha-1) r\left(z_{i} \mid \nu\right) y_{i}\right\} \\
U_{\nu}(\boldsymbol{\varphi})=- & \frac{\alpha}{2} \sum_{i=1}^{n}\left(1-d_{i}\right)\left\{\psi\left(\frac{\nu+1}{2}\right)-\psi\left(\frac{\nu}{2}\right)-b_{01}\left(c_{i} \mid \nu\right)\right. \\
& \left.-\frac{c_{i} r\left(c_{i} \mid \nu\right)}{\nu}\right\} R\left(c_{i} \mid \nu\right)+\frac{1}{2} \sum_{i=1}^{n} d_{i}\left\{\psi\left(\frac{\nu+1}{2}\right)-\psi\left(\frac{\nu}{2}\right)-\frac{1}{\nu}\right. \\
& \left.-\log \left(1+\frac{z_{i}^{2}}{\nu}\right)+\left(\frac{\nu+1}{\nu}\right)\left(1+\frac{z_{i}^{2}}{\nu}\right)^{-1} \frac{z_{i}^{2}}{\nu}\right\} \\
& +\frac{\alpha-1}{2} \sum_{i=1}^{n} d_{i}\left\{\psi\left(\frac{\nu+1}{2}\right)-\psi\left(\frac{\nu}{2}\right)-b_{01}\left(z_{i} \mid \nu\right)\right. \\
& \left.\quad-\frac{z_{i} r\left(z_{i} \mid \nu\right)}{\nu}\right\} \\
U_{\alpha}(\boldsymbol{\varphi})= & \sum_{i=1}^{n}\left(1-d_{i}\right) \log \left\{1-T\left(c_{i} \mid \nu\right)\right\}+\sum_{i=1}^{n} d_{i}\left\{\frac{1}{\alpha}+\log T\left(z_{i} \mid \nu\right)\right\}
\end{aligned}
$$

em que $r(x \mid \nu)=t(x \mid \nu) / T(x \mid \nu), R(x \mid \nu)=T(x \mid \nu) /(1-T(x \mid \nu)), \psi(x)=\frac{d}{d x} \log \Gamma(x)$ é a função digama e $b_{m n}\left(c_{m} \mid \nu+m\right)$ é o momento truncado definido em (3.29).

Note-se que, se $\alpha=1$ as equações (3.43)-(3.45) são reduzidas às funções escore do modelo de regressão $t$ de Student censurado, enquanto que, se $\alpha=1$ e $\nu \rightarrow \infty$, então $r(c \mid \nu) \rightarrow r(c)=$ $\phi(c) / \Phi(c), R(c \mid \nu) \rightarrow R(c)=\Phi(c) /(1-\Phi(c))$, e $U(\nu) \rightarrow 0$, portanto, as equações (3.43)-(3.44) se reduzem às funções escore de modelo Tobit usual.

Os elementos da matrix de informação observada $\boldsymbol{J}(\boldsymbol{\varphi})$ para o modelo PT-CR denotados por $j_{\boldsymbol{\varphi}_{i} \boldsymbol{\varphi}_{j}}$, podem ser obtidos computando a segunda derivada parcial de função de logverossimilhança (3.42), isto é, $j_{\boldsymbol{\varphi}_{i} \boldsymbol{\varphi}_{j}}=-\partial^{2} \ell\left(\boldsymbol{\varphi} ; \mathbf{Y}^{o}\right) / \partial \boldsymbol{\varphi}_{i} \partial \boldsymbol{\varphi}_{j}$, enquanto que a matriz de informação esperada é obtida como $\boldsymbol{I}(\boldsymbol{\varphi})=\mathrm{E}[\boldsymbol{J}(\boldsymbol{\varphi})]$, o qual envolve o computo de valores esperados truncados que não tem forma fechada e deveriam ser obtidos numéricamente. O Apêndice B.4 apresenta as expressões para os elementos das matrizes $\boldsymbol{I}(\boldsymbol{\varphi})$ e $\boldsymbol{J}(\boldsymbol{\varphi})$. 
A matriz de informação esperada $\boldsymbol{I}(\boldsymbol{\theta})$ da parametrização original $\boldsymbol{\theta}=(\boldsymbol{\beta}, \eta, \alpha, \nu)^{\top}$ pode ser recuperada usando $\boldsymbol{I}(\boldsymbol{\theta})=(\partial \boldsymbol{\varphi} / \partial \boldsymbol{\theta})^{\top} \boldsymbol{I}(\boldsymbol{\varphi})(\partial \boldsymbol{\varphi} / \partial \boldsymbol{\theta})$, em que

$$
\frac{\partial \boldsymbol{\varphi}}{\partial \boldsymbol{\theta}}=\left(\begin{array}{cccc}
\frac{1}{\eta} \mathbf{I}_{p} & -\frac{1}{\eta^{2}} \boldsymbol{\beta} & 0 & 0 \\
0 & -\frac{1}{\eta^{2}} & 0 & 0 \\
0 & 0 & 1 & 0 \\
0 & 0 & 0 & 1
\end{array}\right)
$$

Finalmente, o estimador de máxima verossimilhança para $\boldsymbol{\theta}=(\boldsymbol{\beta}, \eta, \alpha, \nu)^{\top}$ pode ser obtido usando métodos iterativos baseados no algoritmo de Newton-Rapshon a partir da função escore (3.43) - (3.46) e aplicando a transformação inversa $\boldsymbol{\beta}=\gamma / \sigma$ e $\eta=1 / \sigma$. As estimativas das variâncias dos estimadores podem ser obtidas avaliando a inversa da matriz de informação observada $\boldsymbol{J}^{-1}(\boldsymbol{\varphi})$ nos estimadores de máxima verossimilhança $\widehat{\varphi}=(\widehat{\gamma}, \widehat{\sigma}, \widehat{\alpha}, \widehat{\nu})^{\top}$ e pelo uso do resultado acima.

\subsection{Seleção de Modelos e Análise de Resíduos}

Nesta seção propomos alguns critérios para a seleção do modelo ajustado e uma metodologia para a análise dos resíduos.

\subsubsection{Seleção de Modelos}

Existem muitas ferramentas de seleção de modelos que são geralmente utilizadas, tais como o Critério de Informação de Akaike (AIC), (Akaike, 1974), o Critério de informação Bayesiano (BIC) (Schwarz, 1978), e o AIC corrigido (AICc) (Sugiura, 1978), que são definidos por

$$
-2 \ell(\boldsymbol{\theta} \mid \mathbf{y})+c(k, n)
$$

em que o termo $c(k, n)$ é uma quantidade que depende do número de parâmetros livres que são estimados no modelo e $k$, é o número de observações na amostra $n$. Para o AIC temos que $c(k, n)=2 k$, enquanto que para o BIC temos que $c(k, n)=k \log (n)$. Para o AICc temos que $c(k, n)=2 k(k+1) /(n-(k+1))$.

Para a escolha do melhor modelo, nós usaremos os critérios AIC, BIC e AICc, isto é, o melhor modelo é aquele com o menor AIC, BIC ou AICc.

\subsubsection{Análise de resíduos}

A análise de resíduos tem como finalidade detectar a presença de observações atípicas e avaliar as suposições do modelo, podendo incluir testes formais para detectar afastamentos das suposições do modelo considerado, bem como gráficos informais para apresentar características gerais dos resíduos.

Seguindo Garay et al. (2017) e Arellano-Valle et al. (2012), neste trabalho considerou-se os resíduos martingais transformados $r_{M T i}$ propostos por Barros et al. (2010) como ferramenta de diagnóstico para avaliar afastamantos do modelo postulado para a variável resposta, bem como detectar a presença de observações atípicas. Os resíduos são definidos como

$$
r_{M T i}=\operatorname{sign}\left(r_{M i}\right) \sqrt{-2\left[r_{M i}+\delta_{i} \log \left(\delta_{i}-r_{M_{i}}\right)\right]}, \quad i=1, \ldots, n
$$

em que $r_{M i}=\delta_{i}+\log S\left(y_{i} ; \widehat{\boldsymbol{\theta}}\right)$ é o resíduo martingal proposto por Ortega et al. (2003), em que $\delta_{i}=0,1$ indica se a $i$-ésima observação é censurada ou não, respectivamente, $\operatorname{sign}\left(r_{M i}\right)$ denota o 
signal de $r_{M i}$ e $S\left(y_{i} ; \widehat{\boldsymbol{\theta}}\right)=P_{\widehat{\boldsymbol{\theta}}}\left(Y_{i}>y_{i}\right)$ representa a função de sobrevivência avaliada em $y_{i}$, em que $\widehat{\boldsymbol{\theta}}$ representa as estimações de MV para $\boldsymbol{\theta}$.

Como surgerido por Garay et al. (2017), usamos este tipo de resíduos padronizados devido ao fato que são simétricamente distribuídos ao redor de zero, o que facilitará a construção dos envelopes simulados com pouco esforço computacional e será útil para detectar uma especificação incorreta do modelo, assim como a presença de observações atípicas.

\subsection{Simulação}

A fim de estudar o desempenho dos estimadores de máxima verossimilhança $\widehat{\boldsymbol{\beta}}, \widehat{\eta}, \widehat{\alpha}$ e $\widehat{\nu}$ de $\boldsymbol{\beta}$, $\eta, \alpha$ e $\nu$, respectivamente; apresentamos um estudo de simulação de Monte Carlo com tamanhos de amostras $n=150,300,750$, e 1000. Consideramos o modelo PT-CR definido na Seção 3.5 com $\mathbf{x}_{i}^{\top}=\left(1, x_{i}\right)$ para $i=1, \ldots, n$. Os verdadeiros valores dos parâmetros foram tomados como $\boldsymbol{\beta}=(2,1.5)^{\top}, \eta=1.5, \alpha=0.4,2.5$ e $\nu=3$. Consideramos também níveis de censura iguais a $p=0,10,25$ e $45 \%$. A covariável $x_{i}$ foi gerada segundo uma distribuição uniforme $\mathrm{U}(0.1,20)$ como considerado em Garay et al. (2017). Para cada combinação de parâmetros, tamanhos de amostras e níveis de censura, foram geradas 2000 amostras do modelo PT-CR com erros $\epsilon_{i} \sim \mathrm{PT}(0, \eta, \alpha, \nu)$. Para avaliar o desempenho dos estimadores foram considerados o viés relativo (VR) em valor absoluto e a raiz quadrada do erro quadrático médio (REQM). Eles são dados por

$$
\operatorname{VR}\left(\widehat{\theta}_{i}\right)=\frac{1}{2000} \sum_{j=1}^{2000}\left(\frac{\widehat{\theta}_{i}^{(j)}}{\theta_{i}}-1\right), \operatorname{REQM}\left(\widehat{\theta}_{i}\right)=\sqrt{\frac{1}{2000} \sum_{j=1}^{2000}\left(\widehat{\theta}_{i}^{(j)}-\theta_{i}\right)^{2}}
$$

respectivamente, em que $\widehat{\theta}_{i}^{(j)}$ é o estimador de $\theta_{i}$ para a $j$-ésima amostra, para $\theta_{i} \in \boldsymbol{\theta}=$ $\left(\boldsymbol{\beta}^{\top}, \eta, \alpha, \nu\right)^{\top}$.

Os estimadores de máxima verossimilança dos parâmetros foram computados usando a função optim do pacote estatístico R. A optimização da função de verossimilhança foi feita usando métodos iterativos baseados no algoritmo de Newton-Rapshon usando as funções escore. Pode-se observar da Tabela A.1 no Apêndice A que o VR e a REQM tendem a diminuir o valor de $n$ aumenta, indicando que estimativas baseadas no método de máxima verossimilhança têm boas propriedades assintóticas. Esse padrão é o mesmo para os diferentes níveis de censura de $p$ sob consideração.

\subsection{Aplicação}

Nesta seção, ilustramos o método desenvolvido considerando os dados descritos por Mroz (1987). O conjunto de dados referentes a Salários, é composto por 753 mulheres brancas casadas com idade entre 30 e 60 anos em 1975, em que 428 mulheres trabalharam em algum momento desse ano.

A variável de resposta $y$ usada nesta aplicação é o rendimento médio por hora. Se o valor do rendimento for igual a zero, significa que essas mulheres não trabalharam no ano de estudo, assim essas observações serão consideradas como observações censuradas em zero. (censura à esquerda).

As covariáveis consideradas foram a idade da mulher $\left(X_{1}\right)$, anos de escolaridade $\left(X_{2}\right)$, o número de crianças menores de seis anos na casa $\left(X_{3}\right)$ e o número de crianças entre seis e dezenove anos de idade $\left(X_{4}\right)$. Esses dados foram analisados anteriormente por Arellano-Valle et al. (2012) usando um modelo de regressão $t$ de Student censurado, e mais tarde por Garay et al. (2017) 
Tabela 3.5: Resumo estatístico para os dados de salários.

\begin{tabular}{lccccc}
\hline & $n$ & Média & EP & Assimetria & Curtose \\
\hline Dados completos & 753 & 2.3746 & 3.2418 & 2.7778 & 15.7967 \\
Dados não censurados & 428 & 4.1777 & 3.3103 & 3.0801 & 16.8212 \\
\hline
\end{tabular}

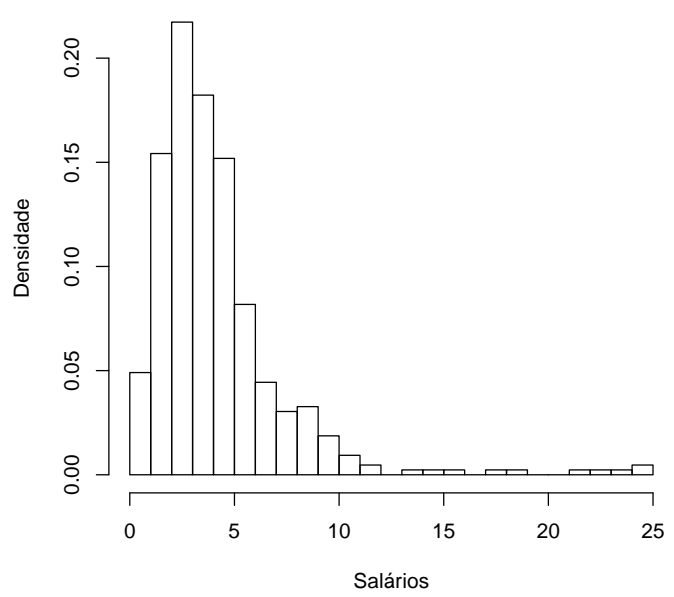

Figura 3.5: Histograma dos Salários, dados de Mroz.

usando os modelos SMN-CR (Scale Mixture of Normal Censored Regression Model) Garay et al. (2017).

Nós analisamos os conjunto de dados descritos por Mroz (1987) ajustando um modelo PT$\mathrm{CR}$, e para comparar nossa proposta com outros métodos existentes na literatura, ajustamos os seguintes modelos SMN-CR propostos por Garay et al. (2017): T-CR (Student-t censored regression model), SL-CR (Slash censored regression model), e N-CR (normal censored regression model), isto é, o modelo Tobit usual.

A Tabela 3.5 mostra os coeficientes de assimetria e curtose para dados completos, bem como para as observações não censuradas. Pode-se observar que os valores dos coeficientes de assimetria e curtose justificam o uso de um modelo que seja capaz de acomodar alto grau de assimetria, por exemplo, o modelo PT-CR, além disso, o histograma das observações não censuradas dado na Figura 3.5 também suporta essa escolha.

A Tabela 3.6 apresenta as estimativas dos parâmetros com seus correspondentes erros padrão (EP) para os modelos PT-CR, T-CR, SL-CR e N-CR. A Tabela 3.7 apresenta alguns critérios de seleção de modelos juntamente com os valores da log-verossimilhança. Pode-se notar que, segundo os criterios AIC, BIC e AICc, o modelo PT-CR parece produzir melhor ajuste aos dados sob salários de Mroz que os modelos SMN-CR e que o modelo Tobit usual (model N-CR), apoiando afirmação de afastamento de erros simétricos. Além disso, o EP das estimativas dos parâmetros obtidos no modelo PT-CR é menor do que o DP das estimativas dos parâmetros obtidos com os modelos SMN-CR (modelos T-CR e SL-CR) e N-CR (modelo Tobit usual).

Uma indicação mais categórica de que um modelo assimétrico deveria ser considerado, resulta de testar a hipótese de um modelo simétrico (T-CR) contra um modelo assimétrico, isto é, um 
Tabela 3.6: Estimativas de MV dos parâmetros e erros padrões (EP) obtidos no ajuste dos modelos PT-CR, T-CR, SL-CR e N-CR para os dados de salários.

\begin{tabular}{lrrrrrrrr}
\hline & \multicolumn{2}{c}{ PT-CR } & \multicolumn{2}{c}{ T-CR } & \multicolumn{2}{c}{ SL-CR } & \multicolumn{2}{c}{ N-CR } \\
$\boldsymbol{\theta}$ & Estimativa & EP & Estimativa & EP & Estimativa & EP & Estimativa & EP \\
\hline$\beta_{0}$ & -8.8412 & 0.3785 & -1.0474 & 1.1549 & -1.1445 & 1.4377 & -2.7510 & 1.7334 \\
$\beta_{1}$ & -0.1331 & 0.0004 & -0.1108 & 0.0204 & -0.1084 & 0.0232 & -0.1046 & 0.0276 \\
$\beta_{2}$ & 0.4887 & 0.0050 & 0.6475 & 0.0658 & 0.6434 & 0.0635 & 0.7281 & 0.0831 \\
$\beta_{3}$ & -3.4010 & 0.1869 & -3.1636 & 0.3878 & -3.0958 & 0.3785 & -3.0264 & 0.4406 \\
$\beta_{4}$ & -0.3053 & 0.0149 & -0.2964 & 0.1247 & -0.2946 & 0.1210 & -0.2143 & 0.1527 \\
$\eta$ & 4.1538 & 0.1320 & 3.2616 & 0.1731 & 2.5958 & 0.3071 & 4.5760 & 0.1697 \\
$\alpha$ & 12.8760 & 0.9871 & - & - & - & - & - & - \\
$\nu$ & 6.5899 & 0.9014 & 4.2000 & 0.8068 & 1.4061 & 0.4361 & - & - \\
\hline
\end{tabular}

Tabela 3.7: Critérios de seleção dos modelos ajustados aos dados de salários.

\begin{tabular}{lrrrr}
\hline Critério & PT-CR & T-CR & SL-CR & N-CR \\
\hline$\ell(\boldsymbol{\theta})$ & -1413.942 & -1440.145 & -1436.286 & -1481.655 \\
AIC & 2843.883 & 2894.291 & 2886.573 & 2975.311 \\
AICc & 2828.077 & 2880.441 & 2872.723 & 2963.424 \\
BIC & 2880.876 & 2926.659 & 2918.941 & 3003.055 \\
\hline
\end{tabular}

modelo PT-CR, através das hipóteses

$$
H_{0}: \alpha=1 \quad \text { versus } \quad H_{1}: \alpha \neq 1 \text {, }
$$

usando a razão de verossimilhanças

$$
\Lambda=\frac{L_{T-C R}(\widehat{\boldsymbol{\theta}})}{L_{P T-C R}(\widehat{\boldsymbol{\theta}})}
$$

a qual para os dados em estudo produz o valor observado

$$
-2 \log \Lambda=52.41
$$

que é maior que o valor crítico da chi-quadrado ao $5 \%$ com um grau de liberdade dado por $\chi_{1,95 \%}^{2}=3.84$. Esta é uma indicação de que o modelo PT-CR parece ajustar melhor os dados de salários que o modelo T-CR.

Finalmente, com o objetivo de verificar se existe alguma especificação incorrecta nas suposições do modelo ajustado, os gráficos dos envelopes simulados para os resíduos tipo martingais transformados são mostrados na Figura 3.6. Essa figura indica que o modelo PT-CR é, aparentemente, mais indicado para o ajuste destes dados do que os modelos SMN-CR. Também pode-se observar que os modelos SMN-CR com caudas mais pesadas ajustam melhor os dados do que o modelo N-CR, uma vez que há poucas observações que se encontram fora dos envelopes. 


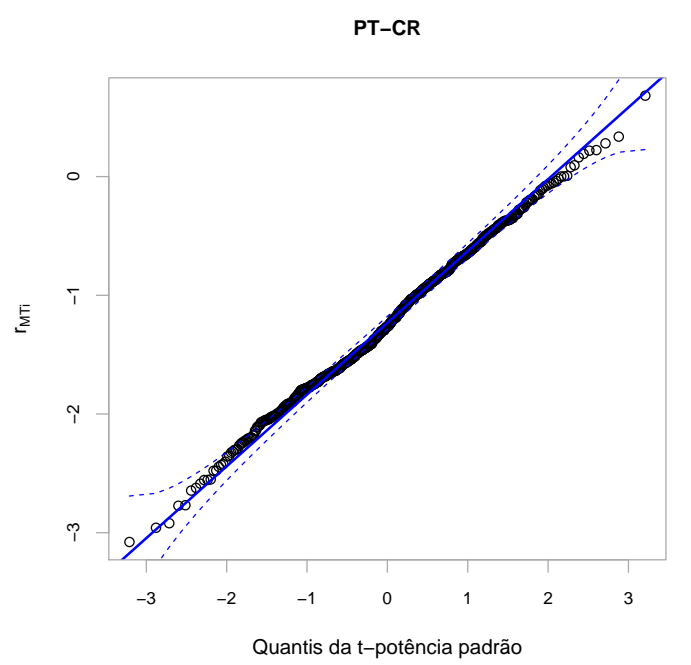

(a)

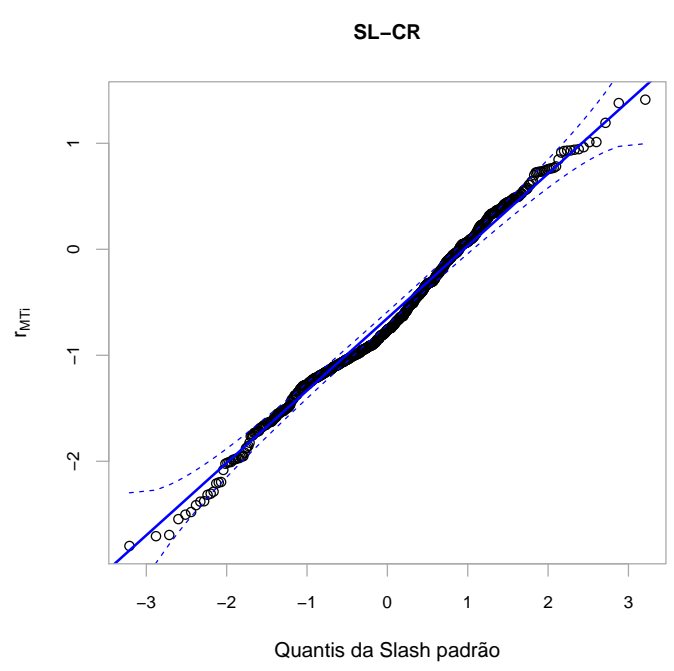

(c)

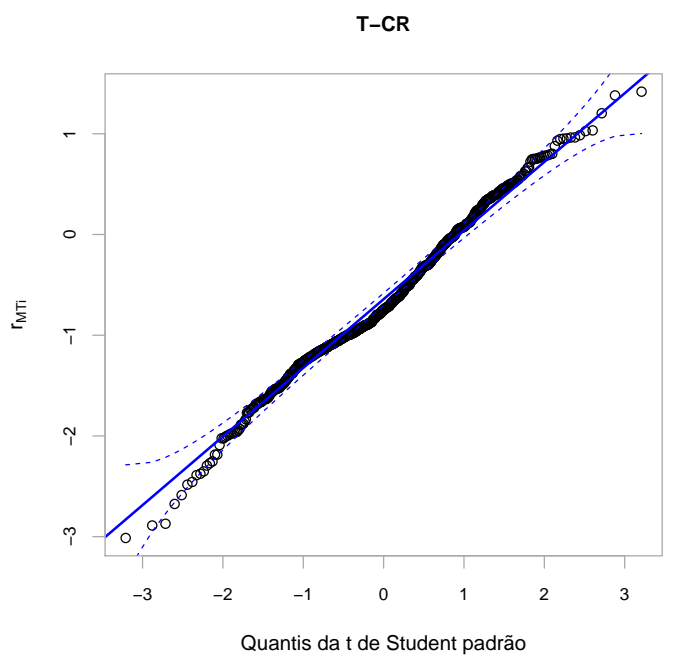

(b)

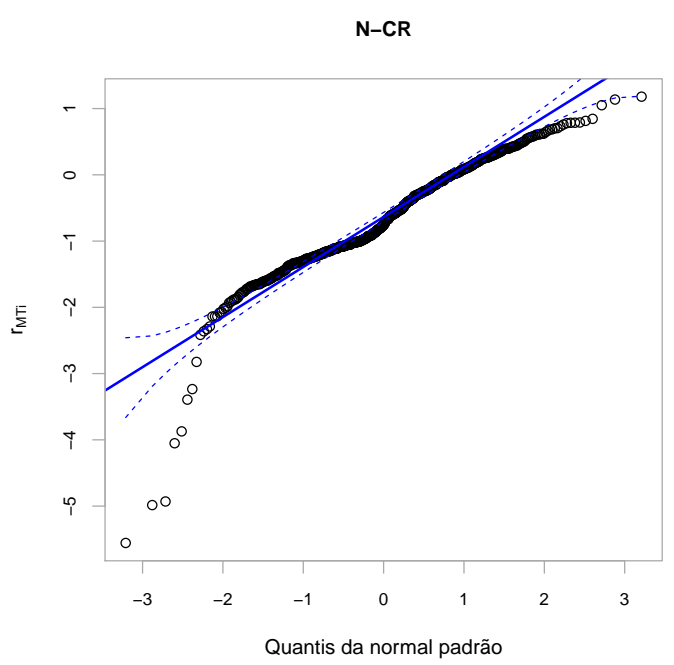

(d)

Figura 3.6: Envelopes dos resíduos tipo martingais transformados $r_{M T i}$, para (a) Modelo PT-CR. (b) Modelo T-CR. (c) Model SL-CR. (d) Modelo N-CR.

\subsection{Conclusões}

Neste capítulo, introduzimos dois novos modelos assimétricos úteis para a modelagem de dados com alto grau de assimetria e coeficiente de curtose maior (ou menor) do que é possível obter com o modelo normal.

O primeiro modelo assimétrico (modelo PST) é baseado na combinação dos modelos $t$ assimétrico por Azzalini e Capitanio (2003) e $t$-potência por Zhao e Kim (2016). Este novo modelo apresenta amplos intervalos de assimetria e curtose, os quais são muito úteis para modelas dados assimétricos e de caudas pesadas. O problema da estimação dos parâmetros no modelo é resolvido usando o método de máxima verossimilhança, que é também usado para estudar as propriedades assintóticas dos estimadores sob a teoria de grandes amostras. A matriz de informa- 
ção observada é analiticamente obtida e mostrada ser não singular, o que garante a validade dos resultados para estatísticas de razão de verossimilhanças com grandes amostras. Os elementos da função escore são também apresentados. Para este modelo proposto, uma aplicação a dados sobre altitude de vulcões no mundo ilustra o fato de que o novo modelo pode ser útil em aplicações reais.

O segundo modelo proposto (modelo PT-CR) é uma alternativa para o modelo de regressão $t$ de Student censurado (modelo T-CR). O modelo é baseado na nova família de distribuições assimétricas e de caudas pesadas $t$-potência. Mais ainda, o modelo de regressão ordinário Tobit e o modelo de regressão $t$-Student censurado são casos especiais. Os elementos da matriz de informação observada e esperada são analiticamente obtidos permitindo a implementação direta da inferência neste tipo de modelos.

O problema da estimação dos parâmetros é tratado usando o método de máxima verossimilhança; e estatísticas de razão de verossimilhança baseados nas propriedades assintóticas dos estimadores para grandes amostras podem ser usados para teste de hipótese. Um estudo de simulação em pequena escala indica boa recuperação de parâmetros com a abordagem de estimativa desenvolvida. Uma aplicação para ilustrar a nossa proposta é apresentada e concluímos que o método proposto pode ser uma alternativa útil a outros modelos existentes na literatura, tais com o modelo T-CR de Arellano-Valle et al. (2012) e a classe de modelos de regressão de escala de mistura normais (SMN-CR) proposta por Garay et al. (2017). 


\section{Capítulo 4}

\section{Modelos Lineares Mistos Normal-Potência}

\subsection{Introdução}

Como discutido no Capítulo 1, a suposição de normalidade dos efeitos aleatórios e os erros é muito comum em modelos lineares mistos (MLM's), mas esta suposição pode não ser adequada e pode levar a consequências prejudiciais para a inferência. Segundo alguns autores (veja, por exemplo Arellano-Valle et al., 2005; Butler e Louis, 1992; Verbeke e Lasaffre, 1997), apesar de que a inferência nos efeitos fixos pode ser robusta à não normalidade dos efeitos aleatórios, podese estar interessado na eficiência e na validade de inferência nos efeitos individuais. Mais ainda, estimação dos efeitos aleatórios menos restritivas pode fornecer considerável discernimento, por exemplo, a exclusão de covariáveis importantes pode ser sugerida pela assimetria na variável resposta revelando características críticas da heterogeneidade presente entre sujeitos (Lachos , 2004).

Recentemente, uma variedade de métodos têm sido propostos para relaxar a suposição de normalidade, por exemplo, Zhang e Davidiam (2001) usaram a representação semi-não-paramétrica proposta por Gallant e Nychka (1987) para aproximar a densidade dos efeitos aleatórios e fornecer flexibilidade na captura de uma ampla gama de comportamentos não normais. Pinheiro et al. (2001) propuseram um modelo linear hierárquico de efeitos mistos robusto assumindo que os efeitos aleatórios e os erros dentro dos sujeitos seguem distribuições $t$ multivariadas.

Arellano-Valle et al. $(2005,2007)$ usaram a distribuição normal-assimétrica para propor o modelo linear misto normal assimétrico (SNLMM). Aqui, os autores desenvolveram um algoritmo tipo EM para a inferência via máxima verossimilhança em abordagem clássica, e também, em uma perspectiva bayesiana (Arellano-Valle et al., 2007) derivaram um esquema MCMC (Markov Chain Monte Carlo) para realizar a inferência. A distribuição normal-assimétrica multivariada foi considerada tembém por Lin e Lee (2008) com um algoritmo híbrido eficiente para a obtenção de estimativas de máxima verossimilhança dos parâmetros. Rosa et al. (2003) realizaram análise a posteriori em modelos lineares de efeitos mistos com distribuições normais e independentes usando um esquema MCMC em uma abordagem bayesiana. Recentemente Lachos et al. (2010) apresentou uma nova classe de modelos lineares assimétricos baseados na distribuição normalassimétrica e independentes.

Neste capítulo, nós introduzimos um novo método que é particularmente atraente em modelos lineares mistos assumindo que ambos, os efeitos aleatórios e o termo de erro no modelo seguem distribuições normal-potência. A suposição de distribuição normal-potência da flexibilidade para acomodar formas assimétricas à direita e à esquerda para os efeitos aleatórios e os erros. Curtose maior ou menor do que o usual modelo normal pode ser também acomodada. 
O plano deste capítulo é como segue. Na Seção 4.2 introduzimos a versão univariada da proposta, considerando que tanto os erros como os efeitos aleatórios seguem uma distribuição normal-potência Durrans (1992). Chamamos este modelo de modelo com interceptos aleatórios normal-potência. Fazemos também um estudo detalhado do procedimento de estimação nesta classe de modelos, derivamos os dois primeiros momentos da variável resposta, e propomos uma metodologia para obter as predições dos interceptos aleatórios. Na Seção 4.3 generalizamos os resultados obtidos para o caso multivariado. Na Seção 4.4 apresentamos um estudo de simulação em que mostramos que o método de estimação proposto oferece boas propriedades assintóticas dos estimadores dos parâmetros. Na Seção 4.5 apresentamos uma aplicação do método proposto a um conjuto de dados de colesterol previamente analisado por Zhang e Davidiam (2001) usando técnicas semi-paramétricas, e por Arellano-Valle et al. (2005) usando modelos lineares mistos normal-assimétrico. Finalmente, na Seção 4.6 discutimos as principais conclusões do método proposto.

\subsection{O Modelo com Interceptos Aleatórios Normal-Potência}

Seja $Y_{i j}$ o valor da $j$-ésima resposta medida para a $i$-ésima unidade amostral (cluster), para $j=1, \ldots, m_{i}$ e $i=1, \ldots, n$. Consideraremos o seguinte modelo com intercepto aleatório

$$
\begin{aligned}
& Y_{i j}=\mathbf{x}_{i j}^{\top} \boldsymbol{\beta}+\gamma_{i}+\epsilon_{i j}, \quad \text { com } \\
& \gamma_{i} \stackrel{\text { iid }}{\sim} \operatorname{PN}\left(0, \eta_{g}, \alpha_{g}\right) \quad \text { e } \quad \epsilon_{i j} \stackrel{\text { ind }}{\sim} \operatorname{PN}\left(0, \eta_{e}, \alpha_{e}\right),
\end{aligned}
$$

em que $\gamma_{i}$ é independente de $\epsilon_{i j}, \mathbf{x}_{i j}=\left(x_{i j 1}, \ldots, x_{i j p}\right)^{\top}$ é um vetor conhecido que contem os valores das variáveis explicativas associadas aos efeitos fixos, e $\boldsymbol{\beta}=\left(\beta_{1}, \ldots, \beta_{p}\right)^{\top}$ é um vetor de parâmetros descohecidos de componentes sistemáticos. Esta suposição leva ao modelo hierárquico:

$$
\begin{aligned}
Y_{i j} \mid \gamma_{i} & \stackrel{\text { ind }}{\sim} \mathrm{PN}\left(\mathbf{x}_{i j}^{\top} \boldsymbol{\beta}+\gamma_{i}, \eta_{e}, \alpha_{e}\right), \\
\gamma_{i} & \stackrel{\mathrm{iid}}{\sim} \mathrm{PN}\left(0, \eta_{g}, \alpha_{g}\right),
\end{aligned}
$$

$i=1, \ldots, n$ e $j=1, \ldots, m_{i}$. No modelo (4.2), pode-se notar que, se $\alpha_{g}=\alpha_{e}=1$, se obtem o modelo linear misto ordinario com ambos, o intercepto aleatório e termo do erro distribuidos normalmente. Por outro lado, $\operatorname{com} \alpha_{e}=1$, o modelo normal com intercepto aleatório normalpotência segue. $\mathrm{O}$ modelo normal-potência com intercepto aleatório normal é também um caso especial tomando $\alpha_{g}=1$.

\subsubsection{Momentos}

Seguindo a abordagem marginal apresentada para o caso do modelo linear normal de efeitos mistos apresentada na Seção 2.2.1, junto com a equação (2.15), é possível obter a média, a variância, a covariância e a correlação da distribuição marginal de $Y_{i j}$, descritas a seguir. 


\section{Média}

A média marginal de $Y_{i j}$ é calculada baseados na esperança condicional de $Y_{i j}$ dado o efeito aleatório $\gamma_{i}$, isto é,

$$
\begin{aligned}
\mathrm{E}\left[Y_{i j}\right] & =\mathrm{E}\left[\mathrm{E}\left(Y_{i j} \mid \gamma_{i}\right)\right] \\
& =\mathrm{E}\left[\mathbf{x}_{i j}^{\top} \boldsymbol{\beta}+\gamma_{i}+\mathrm{E}\left(\epsilon_{i j}\right)\right] \\
& =\mathrm{E}\left[\mathbf{x}_{i j}^{\top} \boldsymbol{\beta}+\gamma_{i}+\eta_{e} \int_{0}^{1} \alpha_{e} \Phi^{-1}(v) v^{\alpha_{e}-1} d v\right] \\
& =\mathbf{x}_{i j}^{\top} \boldsymbol{\beta}+\eta_{g} \int_{0}^{1} \alpha_{g} \Phi^{-1}(u) u^{\alpha_{g}-1} d u+\eta_{e} \int_{0}^{1} \alpha_{e} \Phi^{-1}(v) v^{\alpha_{e}-1} d v \\
& =\mathbf{x}_{i j}^{\top} \boldsymbol{\beta}+\mu_{\gamma}+\mu_{\epsilon}
\end{aligned}
$$

em que $\mu_{\gamma}=\eta_{g} m_{1}$ e $\mu_{\epsilon}=\eta_{e} m_{1}^{\prime}$, com

$$
m_{1}=\int_{0}^{1} \alpha_{g} \Phi^{-1}(u) u^{\alpha_{g}-1} d u \quad \text { e } \quad m_{1}^{\prime}=\int_{0}^{1} \alpha_{e} \Phi^{-1}(v) v^{\alpha_{e}-1} d v
$$

\section{Variância}

Para calcular a variância marginal de $Y_{i j}$ usamos a propriedade da variância condicional dada por

$$
\operatorname{Var}[Y]=\operatorname{Var}[\mathrm{E}(Y \mid X)]+\mathrm{E}[\operatorname{Var}(Y \mid X)]
$$

em que $X$ e $Y$ são duas variáveis aleatórias. Assim, usando essa expressão obtemos

$$
\begin{aligned}
\operatorname{Var}\left[Y_{i j}\right] & =\operatorname{Var}\left[\mathrm{E}\left(Y_{i j} \mid \gamma_{i}\right)\right]+\mathrm{E}\left[\operatorname{Var}\left(Y_{i j} \mid \gamma_{i}\right)\right] \\
& \left.=\operatorname{Var}\left[\mathbf{x}_{i j}^{\top} \boldsymbol{\beta}+\gamma_{i}+\mu_{\epsilon}\right)\right]+\mathrm{E}\left[\operatorname{Var}\left(\epsilon_{i j}\right)\right] \\
& =\operatorname{Var}\left[\gamma_{i}\right]+\operatorname{Var}\left[\epsilon_{i j}\right] \\
& =\sigma_{\gamma}^{2}+\sigma_{\epsilon}^{2}
\end{aligned}
$$

em que $\sigma_{\gamma}^{2}=\eta_{g}^{2}\left(m_{2}-m_{1}^{2}\right)$ e $\sigma_{\epsilon}^{2}=\eta_{e}^{2}\left(m_{2}^{\prime}-m_{1}^{\prime 2}\right)$, com $m_{1}$ e $m_{1}^{\prime}$ dados acima, e

$$
m_{2}=\int_{0}^{1} \alpha_{g}\left[\Phi^{-1}(u)\right]^{2} u^{\alpha_{g}-1} d u \quad \text { e } \quad m_{2}^{\prime}=\int_{0}^{1} \alpha_{e}\left[\Phi^{-1}(v)\right]^{2} v^{\alpha_{e}-1} d v .
$$

\section{Covariância e correlação}

Assumindo indepêndencia condicional dos $Y_{i j}$ e usando a definição de covariância condicional dada por

$$
\operatorname{Cov}[Y, X]=\operatorname{Cov}[\mathrm{E}(Y \mid Z), \mathrm{E}(X \mid Z)]+\mathrm{E}[\operatorname{Cov}(Y, X \mid Z)]
$$

em que $Y, X$ e $Z$ são variáveis aleatórias, temos que a covariância entre $Y_{i j}$ e $Y_{i j^{\prime}}$, duas observações nos momentos $j$ e $j^{\prime}$ do $i$-ésimo indivíduo, é dada por

$$
\operatorname{Cov}\left[Y_{i j}, Y_{i j^{\prime}}\right]=\operatorname{Cov}\left[\mathrm{E}\left(Y_{i j} \mid \gamma_{i}\right), \mathrm{E}\left(Y_{i j^{\prime}} \mid \gamma_{i}\right)\right]+\mathrm{E}\left[\operatorname{Cov}\left(Y_{i j}, Y_{i j^{\prime}} \mid \gamma_{i}\right)\right]
$$


e pela independência condicional das observações, o termo $\mathrm{E}\left[\operatorname{Cov}\left(Y_{i j}, Y_{i j^{\prime}} \mid \gamma_{i}\right)\right]$ é igual a zero, então a covariância de $Y_{i j}$ fica dada por

$$
\begin{aligned}
\operatorname{Cov}\left[Y_{i j}, Y_{i j^{\prime}}\right] & =\operatorname{Cov}\left[\mathrm{E}\left(Y_{i j} \mid \gamma_{i}\right), \mathrm{E}\left(Y_{i j^{\prime}} \mid \gamma_{i}\right)\right] \\
& =\operatorname{Cov}\left[\mathbf{x}_{i j}^{\top} \boldsymbol{\beta}+\gamma_{i}+\mu_{\epsilon}, \mathbf{x}_{i j^{\prime}}^{\top} \boldsymbol{\beta}+\gamma_{i}+\mu_{\epsilon}\right]+\mathrm{E}[0] \\
& =\operatorname{Cov}\left[\gamma_{i}, \gamma_{i}\right] \\
& =\sigma_{\gamma}^{2},
\end{aligned}
$$

Note que as correlações entre as observações $Y_{i j}$ e $Y_{i j^{\prime}}$ para $j \neq j^{\prime}$ são sempre maiores que zero, pois segue que $\operatorname{Corr}\left(Y_{i j}, Y_{i j^{\prime}}\right)=\sigma_{\gamma}^{2} /\left(\sigma_{\gamma}^{2}+\sigma_{\epsilon}^{2}\right)>0$.

\subsubsection{Estimação}

A inferência estatística para o modelo de regressão linear com intercepto aleatório normalpotência proposta neste trabalho é baseada no método de máxima verossimilhança. Sejam $f_{Y \mid \gamma}\left(y_{i j} \mid \gamma_{i} ; \boldsymbol{\beta}, \eta_{e}, \alpha_{e}\right)$ e $f_{\gamma}\left(\gamma_{i} ; \eta_{g}, \alpha_{g}\right)$ as fdp de $Y_{i j} \mid \gamma_{i}$ e $\gamma_{i}$, respectivamente. Então, segue que a fdp marginal do vetor aleatório $\mathbf{Y}_{i}=\left(Y_{i 1}, \ldots, Y_{i m_{i}}\right)^{\top}$, é dada por

$$
f\left(\mathbf{y}_{i} ; \boldsymbol{\theta}\right)=\int_{\mathbb{R}}\left\{\prod_{j=1}^{m_{i}} f_{Y \mid \gamma}\left(y_{i j} \mid \gamma_{i} ; \boldsymbol{\beta}, \eta_{e}, \alpha_{e}\right)\right\} f_{\gamma}\left(\gamma_{i} ; \eta_{g}, \alpha_{g}\right) d \gamma_{i},
$$

em que $\boldsymbol{\theta}=\left(\boldsymbol{\beta}^{\top}, \eta_{e}, \alpha_{e}, \eta_{g}, \alpha_{g}\right)^{\top}$ é o vetor de parâmetros a ser estimado no espaço paramétrico

$$
\boldsymbol{\Theta}=\left\{\boldsymbol{\theta} \in \mathbb{R}^{q} \mid \boldsymbol{\beta} \in \mathbb{R}^{p}, \eta_{e} \in \mathbb{R}^{+}, \alpha_{e} \in \mathbb{R}^{+}, \eta_{g} \in \mathbb{R}^{+}, \alpha_{g} \in \mathbb{R}^{+}\right\}
$$

com $q=p+4$, e a função de verossimilhança para o vetor $\mathbf{Y}=\left(\mathbf{Y}_{1}, \ldots, \mathbf{Y}_{n}\right)^{\top}$ é da forma

$$
L(\boldsymbol{\theta} ; \mathbf{Y})=\prod_{i=1}^{n} \int_{\mathbb{R}}\left\{\prod_{j=1}^{m_{i}} f_{Y \mid \gamma}\left(y_{i j} \mid \gamma_{i} ; \boldsymbol{\beta}, \eta_{e}, \alpha_{e}\right)\right\} f_{\gamma}\left(\gamma_{i} ; \eta_{g}, \alpha_{g}\right) d \gamma_{i},
$$

A densidade marginal (4.3) e a função de verossimilhança (4.4) não tem expressões analíticas fechadas com no caso dos modelos lineares mistos normais, e a limitação na inferência estatística baseada no método da máxima verossimilhança é a avaliação das integrais envolvidas na função de verossimilhança (4.4). Geralmente essa limitação é resolvida usando métodos de integração de Monte Carlo, algoritmos tipo EM e métodos aproximados (Wu, 2010). Neste contexto, os métodos de Monte Carlo usam a quadratura de Gauss-Hermite ou técnicas de integração de Monte Carlo para aproximar a integral na função de verossimilhança (4.4) (veja Pinheiro e Chao, 2006), enquanto que os algoritmos tipo EM maximizam indiretamente a verossimilhança usando métodos numéricos estocásticos ou de Monte Carlo no passo E (Vaida et al., 2007). Por fim, os métodos aproximados são denominados assim pelo fato de usar expansões de Taylor ou aproximações de Laplace para aproximar diretamente a função de verossimilhança.

Neste trabalho, utilizamos o método de integração numérica de Gauss-Hermite descrito na Seção 2.5.1 para a avaliação das integrais envolvidas na função de verossimilhança (4.4). Note-se 
que precisamos avaliar a integral $I_{i}$, em que

$$
\begin{aligned}
I_{i} & =\int_{\mathbb{R}}\left\{\prod_{j=1}^{m_{i}} f_{Y \mid \gamma}\left(y_{i j} \mid \gamma_{i} ; \boldsymbol{\beta}, \eta_{e}, \alpha_{e}\right)\right\} f_{\gamma}\left(\gamma_{i} ; \eta_{g}, \alpha_{g}\right) d \gamma_{i} \\
& =\int_{\mathbb{R}}\left\{\prod_{j=1}^{m_{i}} f_{Y \mid \gamma}\left(y_{i j} \mid \gamma_{i} ; \boldsymbol{\beta}, \eta_{e}, \alpha_{e}\right)\right\} \frac{\alpha_{g}}{\sqrt{2 \pi} \eta_{g}} \exp \left(-\frac{\gamma_{i}^{2}}{2 \eta_{g}^{2}}\right)\left\{\Phi\left(\frac{\gamma_{i}}{\eta_{g}}\right)\right\}^{\alpha_{g}-1} d \gamma_{i}
\end{aligned}
$$

Fazendo a mudança de variável $u_{i}=\gamma_{i} / \sqrt{2} \eta_{g}$, a integral $I_{i}$ fica dada por:

$$
I_{i}=\int_{\mathbb{R}} \prod_{j=1}^{m_{i}} f_{Y \mid \gamma}\left(y_{i j} \mid \sqrt{2} \eta_{g} u_{i} ; \boldsymbol{\beta}, \eta_{e}, \alpha_{e}\right)\left\{\Phi\left(\sqrt{2} u_{i}\right)\right\}^{\alpha_{g}-1} \frac{\alpha_{g} \exp \left(-u_{i}^{2}\right)}{\sqrt{\pi}} d u_{i}
$$

que tem a forma (2.23). Usando o método da quadratura de Gauss-Hermite a integral $I_{i}$ pode ser aproximada como:

$$
I_{i} \approx \sum_{k=1}^{Q} \prod_{j=1}^{m_{i}} f_{Y \mid \gamma}\left(y_{i j} \mid \sqrt{2} \eta_{g} u_{k} ; \boldsymbol{\beta}, \eta_{e}, \alpha_{e}\right)\left\{\Phi\left(\sqrt{2} u_{k}\right)\right\}^{\alpha_{g}-1} \frac{\alpha_{g} \omega_{k}}{\sqrt{\pi}}
$$

em que $u_{k}$ são os pontos da quadratura e $\omega_{k}$ os pesos correspondentes. Portanto, a função de verossimilhança (4.4) pode ser aproximada por:

$$
L(\boldsymbol{\theta} ; \mathbf{Y}) \approx \prod_{i=1}^{n}\left(\sum_{k=1}^{Q} \prod_{j=1}^{m_{i}} f_{Y \mid \gamma}\left(y_{i j} \mid \sqrt{2} \eta_{g} u_{k} ; \boldsymbol{\beta}, \eta_{e}, \alpha_{e}\right)\left\{\Phi\left(\sqrt{2} u_{k}\right)\right\}^{\alpha_{g}-1} \frac{\alpha_{g} \omega_{k}}{\sqrt{\pi}}\right)
$$

e o logaritmo da função de verossimilhança $\ell(\boldsymbol{\theta} ; \mathbf{Y})$ pode ser escrito como:

$$
\ell(\boldsymbol{\theta} ; \mathbf{Y}) \approx \sum_{i=1}^{n} \log \left(\sum_{k=1}^{Q} \prod_{j=1}^{m_{i}} f_{Y \mid \gamma}\left(y_{i j} \mid \sqrt{2} \eta_{g} u_{k} ; \boldsymbol{\beta}, \eta_{e}, \alpha_{e}\right)\left\{\Phi\left(\sqrt{2} u_{k}\right)\right\}^{\alpha_{g}-1} \frac{\alpha_{g} \omega_{k}}{\sqrt{\pi}}\right) .
$$

A função escore aproximada é obtida diferenciando o logaritmo da função de verossimilhança $\ell(\boldsymbol{\theta} ; \mathbf{Y})$ em relação a cada um dos parâmetros e é dada por

$$
\boldsymbol{U}(\boldsymbol{\theta})=\left(\boldsymbol{U}_{\boldsymbol{\beta}}^{\top}(\boldsymbol{\theta}), U_{\eta_{e}}(\boldsymbol{\theta}), U_{\eta_{g}}(\boldsymbol{\theta}), U_{\alpha_{e}}(\boldsymbol{\theta}), U_{\alpha_{g}}(\boldsymbol{\theta})\right)^{\top}
$$

No Apêndice B.5.1 são apresentadas as expressões para os componentes do vetor $\boldsymbol{U}(\boldsymbol{\theta})$. O estimador de máxima verossimilhança de $\boldsymbol{\theta}=\left(\boldsymbol{\beta}^{\top}, \eta_{e}, \alpha_{e}, \eta_{g}, \alpha_{g}\right)^{\top}$ é obtido como solução ao sistema de equações $\boldsymbol{U}(\boldsymbol{\theta})=\mathbf{0}$. Note-se que os estimadores dos componentes de $\boldsymbol{\theta}$ não têm uma expressão fechada e precisam ser obtidos numericamente maximizando (4.7).

Sob as condições usuais de regularidade, o estimador de máxima verossimilhança $\widehat{\boldsymbol{\theta}}$ é assintóticamente normal com média $\boldsymbol{\theta}$ e matriz de covariâncias $\boldsymbol{J}^{-1}(\boldsymbol{\theta})$, isto é,

$$
\sqrt{n}(\widehat{\boldsymbol{\theta}}-\boldsymbol{\theta}) \rightarrow N\left(\mathbf{0}, \boldsymbol{J}^{-1}(\boldsymbol{\theta})\right)
$$

em que $\boldsymbol{J}(\boldsymbol{\theta})$ é a matriz de informação observada, obtida como $\boldsymbol{J}(\boldsymbol{\theta})=-\partial^{2} \ell(\boldsymbol{\theta} ; \mathbf{Y}) / \partial \boldsymbol{\theta} \partial \boldsymbol{\theta}^{\top}$. Os elementos da matriz $\boldsymbol{J}(\boldsymbol{\theta})$ são apresentados no Apêndice B.5.2. Para obter os desvios padrão precisa-se obter também de forma numérica as estimativas dos elementos de $\boldsymbol{J}(\boldsymbol{\theta})$. 


\subsubsection{Predição de efeitos aleatórios}

Além de obter estimativas dos parâmetros da distribuição marginal de $\mathbf{Y}_{i}$, muitas vezes precisa-se obter também as predições dos efeitos aleatórios. Essas predições refletem a variabilidade entre indivíduos e permitem a deteção de perfis especiais, por exemplo, indivíduos atípicos ou grupos de indivíduos que evoluem de forma diferente no tempo (veja Molenberghs e Verbeke, 2005). Também, se quer obter predições dos perfis individuais, as predições dos efeitos aleatórios são necessárias.

A abordagem usada para atribuir valores aos efeitos aleatórios é a metodologia que considera o melhor preditor empírico de Bayes. Assim, no modelo linear misto normal (2.3), por exemplo, a estimação dos efeitos aleatórios é baseada na distribuição a posteriori $f\left(\boldsymbol{\gamma}_{i} \mid \mathbf{y}_{i}, \boldsymbol{\beta}, \boldsymbol{\Sigma}\right)$ dada por

$$
f\left(\boldsymbol{\gamma}_{i} \mid \mathbf{y}_{i}, \boldsymbol{\beta}, \boldsymbol{\Sigma}\right)=\frac{f\left(\mathbf{y}_{i} \mid \boldsymbol{\gamma}_{i}, \boldsymbol{\beta}\right) f\left(\boldsymbol{\gamma}_{i}, \boldsymbol{\Sigma}\right)}{\int_{\mathbb{R}^{q}} f\left(\mathbf{y}_{i} \mid \boldsymbol{\gamma}_{i}, \boldsymbol{\beta}\right) f\left(\boldsymbol{\gamma}_{i}, \boldsymbol{\Sigma}\right) d \boldsymbol{\gamma}_{i}}
$$

Geralmente, $\gamma_{i}$ é predito por meio da média da distribuição a posteriori, chamada de média a posteriori de $\gamma_{i}$. Este preditor é denominado melhor preditor empírico de Bayes e é dado por

$$
\begin{aligned}
\widetilde{\boldsymbol{\gamma}}_{i} & =\mathrm{E}\left[\boldsymbol{\gamma}_{i} \mid \mathbf{Y}_{i}\right] \\
& =\int_{\mathbb{R}^{q}} \boldsymbol{\gamma}_{i} f\left(\boldsymbol{\gamma}_{i} \mid \mathbf{y}_{i}, \boldsymbol{\beta}, \boldsymbol{\Sigma}\right) d \boldsymbol{\gamma}_{i}
\end{aligned}
$$

No modelo de regressão linear com interceptos aleatórios normal-potência, propomos usar essa abordagem para obter o melhor preditor linear dos interceptos aleatórios como segue

$$
\widetilde{\gamma}_{i}=\mathrm{E}\left[\gamma_{i} \mid \mathbf{Y}_{i}\right]=\frac{\int_{\mathbb{R}} \gamma_{i} \prod_{j=1}^{m_{i}} f_{Y \mid \gamma}\left(y_{i j} \mid \gamma_{i} ; \widehat{\boldsymbol{\beta}}, \widehat{\eta}_{e}, \widehat{\alpha}_{e}\right) f_{\gamma}\left(\gamma_{i} ; \widehat{\eta}_{g}, \widehat{\alpha}_{g}\right) d \gamma_{i}}{\int_{\mathbb{R}} \prod_{j=1}^{m_{i}} f_{Y \mid \gamma}\left(y_{i j} \mid \gamma_{i} ; \widehat{\boldsymbol{\beta}}, \widehat{\eta}_{e}, \widehat{\alpha}_{e}\right) f_{\gamma}\left(\gamma_{i} ; \widehat{\eta}_{g}, \widehat{\alpha}_{g}\right) d \gamma_{i}}
$$

em que $i=1, \ldots, n$ e $j=1, \ldots, m_{i}$. As integrais no numerador e denominador de (4.9) são resolvidas usando quadratura de Guass-Hermite, da mesma forma que a integral (4.5).

\subsubsection{Seleção de modelos}

Para a seleção de modelos, propomos avaliar a série de ajustes inspecionando critérios de informação, tais como o Critério de Informação de Akaike Marginal, $m A I C=-2 \ell(\widehat{\boldsymbol{\theta}} \mid \mathbf{Y})+$ $2 p N /(N-p-1)$, em que $p$ é o número de parâmetros estimados no modelo considerado, e $N=\sum_{i=1}^{n} m_{i}$, (veja Vaida e Blanchard, 2005); o Critério de Informação Bayesiano de Schwarz, $B I C_{h}=-2 \ell(\widehat{\boldsymbol{\theta}} \mid \mathbf{Y})+p_{r} \log (n)+p_{f} \log (N)$, em que $p_{r}$ é o número de parâmetros correspondentes à componente aleatória, $p_{f}$ é o número de parâmetros correspondentes à componente fixa e $N=$ $\sum_{i=1}^{n} m_{i}$ (veja Delandre et al., 2014). Propomos também usar o Critério de Informação de Akaike modificado, usualmente chamado de AIC consistente, $C A I C=-2 \ell(\widehat{\boldsymbol{\theta}} \mid \mathbf{Y})+p(1+\log (N))$, e o Critério de Informaçãp de Hannan-Quinn, $H Q I C=-2 \ell(\widehat{\boldsymbol{\theta}} \mid \mathbf{Y})+p \log (\log (N))$, em que $p$ é o número de parâmetros estimados no modelo sendo considerado. O melhor modelo é aquele com o menor mAIC (ou $\mathrm{BIC}_{h}$ ou CAIC ou HQIC).

\subsubsection{Análise de resíduos}

Seguindo Usuga (2013), nós propomos usar o gráfico de probabilidade meio normal com envelope simulado sugerido por Atkinson (1985) e Kutner et al. (2005) como ferramenta de diagnóstico para avaliar o afastamento da ditribuição postulada para a respostas $\mathbf{Y}_{i}$ 's bem com a presença de observações atípicas. 
Para construir o gráfico de probabilidade meio normal com envelope simulado, pode ser usado o resíduo quantil aleatorizado, o resíduo condicional ou o resíduo marginal, os quais são definidos por Usuga (2013) como segue

\section{Resíduo quantil aleatorizado}

Tem como objetivo avaliar a adequação geral do modelo aos dados e é definido como

$$
r_{q i j}=\Phi^{-1}\left(F\left(y_{i j} ; \widehat{\boldsymbol{\beta}}, \widehat{\eta}_{e}, \widehat{\alpha}_{e}, \widetilde{\gamma}_{i}\right)\right),
$$

$i=1, \ldots, n$, em que $\widehat{\boldsymbol{\beta}}, \widehat{\eta}_{e}$ e $\widehat{\alpha}_{e}$ são os estimadores de máxima verossimilhança de $\boldsymbol{\beta}, \eta_{e}$ e $\alpha_{e}$, respectivamente; $\widetilde{\gamma}_{i}$ é o melhor preditor linear de $\gamma_{i} ; \Phi^{-1}$ é a função inversa da função de distribuição acumulada de uma variável aleatória com distribuição normal padrão, e $F\left(y_{i j} ; \widehat{\boldsymbol{\beta}}, \widehat{\eta}_{e}, \widehat{\alpha}_{e}, \widetilde{\gamma}_{i}\right)$ é a função de distribuição acumulada de uma variável aleatória com distribuição normal-potência, isto é,

$$
F\left(y_{i j} ; \widehat{\boldsymbol{\beta}}, \widehat{\eta}_{e}, \widehat{\alpha}_{e}, \widetilde{\gamma}_{i}\right)=\left\{\Phi\left(\frac{y_{i j}-\mathbf{x}_{i j}^{\top} \widehat{\boldsymbol{\beta}}-\widetilde{\gamma}_{i}}{\widehat{\eta}_{e}}\right)\right\}^{\widehat{\alpha}_{e}}
$$

\section{Resíduo condicional}

Este resíduo incorpora o efeito da presença de observações atípicas e é dado por

$$
r_{c i j}=\frac{y_{i j}-\widehat{\mathrm{E}}\left[Y_{i j} \mid \widetilde{\gamma}_{i}\right]}{\sqrt{\widehat{\operatorname{Var}}\left[Y_{i j} \mid \widetilde{\gamma}_{i}\right]}}
$$

em que $\widehat{\mathrm{E}}\left[Y_{i j} \mid \widetilde{\gamma}_{i}\right]=\mathbf{x}_{i j}^{\top} \widehat{\boldsymbol{\beta}}+\widetilde{\gamma}_{i}+\widehat{\mu}_{\epsilon}, \mathrm{com}$

$$
\widehat{\mu}_{\epsilon}=\widehat{\eta}_{e} \int_{0}^{1} \widehat{\alpha}_{e} \Phi^{-1}(v) v^{\widehat{\alpha}_{e}-1} d v
$$

e $\widehat{\operatorname{Var}}\left[Y_{i j} \mid \widetilde{\gamma}_{i}\right]=\widehat{\sigma}_{\epsilon}^{2}, \mathrm{com}$

$$
\widehat{\sigma}_{\epsilon}^{2}=\widehat{\eta}_{e}^{2}\left[\int_{0}^{1} \widehat{\alpha}_{e}\left[\Phi^{-1}(v)\right]^{2} v^{\widehat{\alpha}_{e}-1} d v-\left(\int_{0}^{1} \widehat{\alpha}_{e} \Phi^{-1}(v) v^{\widehat{\alpha}_{e}-1} d v\right)^{2}\right]
$$

\section{Resíduo marginal}

O resíduo marginal padronizado incorpora o efeito da presença de observações atípicas e pode ser escrito como

$$
r_{m i j}=\frac{y_{i j}-\widehat{\mu}_{i j}}{\widehat{\sigma}_{i j}}
$$

em que $\widehat{\mu}_{i j}=\mathbf{x}_{i j}^{\top} \widehat{\boldsymbol{\beta}}+\widehat{\mu}_{\gamma}+\widehat{\mu}_{\epsilon}$ e $\widehat{\sigma}_{i j}^{2}=\widehat{\sigma}_{\gamma}^{2}+\widehat{\sigma}_{\epsilon}^{2} ; \operatorname{com} \widehat{\mu}_{\epsilon}$ e $\widehat{\sigma}_{\epsilon}^{2}$ dados em (4.12) e (4.13), respectivamente, e

$$
\widehat{\mu}_{\gamma}=\widehat{\eta}_{g} \int_{0}^{1} \widehat{\alpha}_{g} \Phi^{-1}(u) u^{\widehat{\alpha}_{g}-1} d u
$$

e

$$
\widehat{\sigma}_{\gamma}^{2}=\widehat{\eta}_{g}^{2}\left[\int_{0}^{1} \widehat{\alpha}_{g}\left[\Phi^{-1}(u)\right]^{2} v^{\widehat{\alpha}_{g}-1} d u-\left(\int_{0}^{1} \widehat{\alpha}_{g} \Phi^{-1}(u) u^{\widehat{\alpha}_{g}-1} d u\right)^{2}\right]
$$


sendo $\widehat{\boldsymbol{\beta}}, \widehat{\eta}_{e}, \widehat{\alpha}_{e}, \widehat{\eta}_{g}$ e $\widehat{\alpha}_{g}$ os estimadores de máxima verossimilhança de $\boldsymbol{\beta}, \eta_{e}, \alpha_{e}, \eta_{g}$ e $\alpha_{g}$, respectivamente; e $\widetilde{\gamma}_{i}$ é o melhor preditor linear de $\gamma_{i}$.

\section{Resíduo aleatório}

Exploram a presença de indivíduos atípicos e são definidos por

$$
r_{a i}=\frac{\widetilde{\gamma}_{i}}{\sqrt{\widehat{\operatorname{Var}}\left[\widetilde{\gamma}_{i}\right]}}
$$

$\operatorname{com} i=1, \ldots, n ; \widetilde{\gamma}_{i}$ é o melhor preditor linear de $\gamma_{i}$ e $\widehat{\operatorname{Var}}\left[\widetilde{\gamma}_{i}\right]$ é a variância estimada da distribuição do efeito aleatório.

\section{Envelope simulado}

Atkinson (1985) e Kutner et al. (2005) sugerem usar o gráfico de probabilidade mitadenormal com envelope simulado como ferramenta de diagnóstico. Esse gráfico pode ser construído para cada um dos resíduos descritos anteriormente e permite identificar observações atípicas e avaliar as suposição sobre a distribuição da variável resposta.

Para a construção do gráfico, dispomos do $k$-ésimo valor ordenado $(k=1, \ldots, n)$ do resíduo versus o correspondente valor esperado da estatística de ordem, em valor absoluto, da distribuição $N(0,1)$ dado por

$$
\Phi^{-1}\left(\frac{k+n-1 / 8}{2 n+1 / 2}\right)
$$

em que $\Phi(\cdot)$ é a função de distribuição acumulada da $N(0,1)$.

Para a construção do gráfico meio-normal com envelope simulado seguimos os seguintes passos

1. Simule uma amostra de $n$ observações independentes para a variável resposta normalpotência usando a informação do modelo ajustado aos dados originais.

2. Ajuste às respostas normal-potência simuladas no passo 1 o mesmo modelo ajustado aos dados originais.

3. Calcule o resíduo proposto, e ordene os seus valores absolutos.

4. Repita os passos 1 a $3 k$ vezes.

5. Calcule o mínimo, a média e o máximo dos $k$ resíduos.

6. Disponha no gráfico de probabilidade mitade-normal do resíduo proposto os valores mínimos, médios e máximos dos dados simulados versus os correspondentes valores esperados em (4.16) e conecte os pontos para formar as bandas de confiança.

Segundo Atkinson (1985) é suficiente usar $k=19$. As observações correspondentes aos resíduos fora das bandas de confiança devem ser identificadas e investigadas. A além disso, se há uma proporção considerável de pontos fora das bandas é evidência de que o modelo ajustado não é adequado.

\subsection{O Modelo Linear Misto Normal-Potência}

Nós consideraremos uma alternativa à proposta de Arellano-Valle et al. (2005) assumindo que os vetores aleatórios $\boldsymbol{\gamma}_{i}$ são independentes e identicamente distribuidos $\mathrm{PN}_{q}\left(\mathbf{0}, \boldsymbol{\eta}_{g}, \boldsymbol{\alpha}_{g}\right)$, com 
$\boldsymbol{\eta}_{g}=\operatorname{diag}\left(\eta_{1}^{2} \ldots, \eta_{q}^{2}\right)$ e $\boldsymbol{\alpha}_{g}=\left(\alpha_{1}, \ldots, \alpha_{q}, \alpha\right)$; e para $j=1, \ldots, m_{i}, i=1, \ldots, n$, os $\epsilon_{i j}$ 's sao independentes $\operatorname{PN}\left(0, \eta_{e}, \alpha_{e}\right)$, com $\gamma_{i 1}, \ldots, \gamma_{i q}$ independentes de $\epsilon_{i j}$. Então, esta suposição leva ao modelo hierárquico que chamaremos de modelo linear misto normal-potência, e denotaremos por PNLMM (Power-Normal Linear Mixed Model):

$$
\begin{aligned}
Y_{i j} \mid \boldsymbol{\gamma}_{i} & \stackrel{\operatorname{ind}}{\sim} \mathrm{PN}\left(\mathbf{x}_{i j}^{\top} \boldsymbol{\beta}+\mathbf{w}_{i j}^{\top} \boldsymbol{\gamma}_{i}, \eta_{e}, \alpha_{e}\right), \\
\boldsymbol{\gamma}_{i} & \stackrel{\mathrm{iid}}{\sim} \operatorname{PN}_{q}\left(\mathbf{0}, \boldsymbol{\eta}_{g}, \boldsymbol{\alpha}_{g}\right),
\end{aligned}
$$

Aqui $\mathbf{x}_{i j}^{\top}=\left(x_{i j 1}, \ldots, x_{i j p}\right)$ é a $i j$-ésima linha da matriz $\mathbf{X}_{i}$, e $\mathbf{w}_{i j}^{\top}=\left(w_{i j 1}, \ldots, w_{i j q}\right)$ é a $i j$-ésima linha da matriz $\mathbf{W}_{i}$.

\subsubsection{Momentos}

Seguindo a mesma ideia dos modelos lineares com interceptos normal-potência, temos que os momentos do vetor aleatório $\mathbf{Y}_{i}=\left(Y_{i 1}, \ldots, Y_{i m_{i}}\right)^{\top}$ podem ser obtidos usando a abordagem marginal nos modelos lineares mistos junto com a equação (2.20). Segue que a média de $\mathbf{Y}_{i}$ é dada por

$$
\begin{aligned}
\mathrm{E}\left[\mathbf{Y}_{i}\right] & =\mathrm{E}\left[\mathrm{E}\left(\mathbf{Y}_{i} \mid \boldsymbol{\gamma}_{i}\right)\right] \\
& =\mathrm{E}\left[\mathrm{E}\left(\mathbf{X}_{i} \boldsymbol{\beta}+\mathbf{W}_{i} \boldsymbol{\gamma}_{i}+\boldsymbol{\epsilon}_{i} \mid \boldsymbol{\gamma}_{i}\right)\right] \\
& =\mathrm{E}\left[\mathbf{X}_{i} \boldsymbol{\beta}+\mathbf{W}_{i} \boldsymbol{\gamma}_{i}+\boldsymbol{\mu}_{\epsilon}\right] \\
& =\mathbf{X}_{i} \boldsymbol{\beta}+\mathbf{W}_{i} \boldsymbol{\mu}_{\gamma}+\boldsymbol{\mu}_{\epsilon}
\end{aligned}
$$

em que $\boldsymbol{\mu}_{\gamma}=\boldsymbol{\eta}_{g}^{\frac{1}{2}} \boldsymbol{m}_{g}, \operatorname{com} \boldsymbol{m}_{g}=\left(m_{1}, \ldots, m_{q}\right)^{\top}$ e

$$
m_{k}=\int_{0}^{1} \alpha_{k} \Phi^{-1}(u) u^{\alpha_{k}-1} d u, \quad k=1, \ldots, q
$$

$\boldsymbol{\mu}_{\epsilon}=\mu_{\epsilon} \mathbf{1}_{m_{i}}, \operatorname{com} \mu_{\epsilon}=\eta_{e} m_{e} \mathrm{e}$

$$
m_{e}=\int_{0}^{1} \alpha_{e} \Phi^{-1}(v) v^{\alpha_{e}-1} d v
$$

A variância de $\mathbf{Y}_{i}$ é dada por

$$
\begin{aligned}
\operatorname{Var}\left[\mathbf{Y}_{i}\right] & =\operatorname{Var}\left[\mathrm{E}\left(\mathbf{Y}_{i} \mid \boldsymbol{\gamma}_{i}\right)\right]+\mathrm{E}\left[\operatorname{Var}\left(\mathbf{Y}_{i} \mid \boldsymbol{\gamma}_{i}\right)\right] \\
& =\operatorname{Var}\left[\mathbf{X}_{i} \boldsymbol{\beta}+\mathbf{W}_{i} \boldsymbol{\gamma}_{i}+\boldsymbol{\mu}_{\epsilon}\right]+\mathrm{E}\left[\boldsymbol{\Sigma}_{\epsilon}\right] \\
& =\mathbf{W}_{i}^{\top} \operatorname{Var}\left[\boldsymbol{\gamma}_{i}\right] \mathbf{W}_{i}+\boldsymbol{\Sigma}_{\epsilon} \\
& =\mathbf{W}_{i} \boldsymbol{\Sigma}_{\gamma} \mathbf{W}_{i}^{\top}+\boldsymbol{\Sigma}_{\epsilon}
\end{aligned}
$$

em que $\boldsymbol{\Sigma}_{\gamma}=\boldsymbol{\eta}_{g}^{\frac{1}{2}} \boldsymbol{\Sigma} \boldsymbol{\eta}_{g}^{\frac{1}{2}}$, com

$$
\boldsymbol{\Sigma}=\left(\begin{array}{ccc}
\sigma_{11} & \cdots & \sigma_{1 q} \\
\vdots & \ddots & \vdots \\
\sigma_{q 1} & \cdots & \sigma_{q q}
\end{array}\right)
$$

sendo

$$
\sigma_{k k}=\sigma_{k}^{2}=\int_{0}^{1} \alpha_{k}\left[\Phi^{-1}(u)\right]^{2} u^{\alpha_{k}-1} d u-m_{k}^{2}, \quad k=1, \ldots, q
$$


e

$$
\sigma_{j k}=\alpha(\alpha+1)\left\{\int_{1}^{+\infty} \int_{1}^{+\infty} \frac{\Phi^{-1}\left(u^{-\alpha / \alpha_{j}}\right) \Phi^{-1}\left(v^{-\alpha / \alpha_{k}}\right)}{(u+v-1)^{\alpha+2}} d u d v\right\}-m_{j} m_{k}
$$

para $j, k=1, \ldots, q ; \operatorname{com} j \neq k ;$ e $\boldsymbol{\Sigma}_{\epsilon}=\sigma_{\epsilon}^{2} \mathbf{I}_{m_{i}} \operatorname{com}$

$$
\sigma_{\epsilon}^{2}=\eta_{e}^{2}\left(\int_{0}^{1} \alpha_{e}\left[\Phi^{-1}(u)\right]^{2} u^{\alpha_{e}-1} d u-m_{e}^{2}\right)
$$

\subsubsection{Estimação}

Para fazer inferência estatística no modelo linear misto normal-potência, consideramos novamente o método de máxima verossimilhança. Sejam $f_{Y \mid \boldsymbol{\gamma}}\left(y_{i j} \mid \boldsymbol{\gamma}_{i} ; \boldsymbol{\beta}, \eta_{e}, \alpha_{e}\right)$ e $f_{\boldsymbol{\gamma}}\left(\boldsymbol{\gamma}_{i} ; \boldsymbol{\eta}_{g}, \boldsymbol{\alpha}_{g}\right)$ a fdp de $Y_{i j} \mid \boldsymbol{\gamma}_{i}$ e $\boldsymbol{\gamma}_{i}$, respectivamente, a fdp marginal do vetor aleatório $\mathbf{Y}_{i}=\left(Y_{i 1}, \ldots, Y_{i m_{i}}\right)^{\top}$, é dada por

$$
f\left(\mathbf{y}_{i} ; \boldsymbol{\theta}\right)=\int_{\mathbb{R}^{q}}\left\{\prod_{j=1}^{m_{i}} f_{Y \mid \boldsymbol{\gamma}}\left(y_{i j} \mid \boldsymbol{\gamma}_{i} ; \boldsymbol{\beta}, \eta_{e}, \alpha_{e}\right)\right\} f_{\boldsymbol{\gamma}}\left(\boldsymbol{\gamma}_{i} ; \boldsymbol{\eta}_{g}, \boldsymbol{\alpha}_{g}\right) d \boldsymbol{\gamma}_{i},
$$

em que $\boldsymbol{\theta}=\left(\boldsymbol{\beta}^{\top}, \boldsymbol{\eta}_{g}^{\top}, \boldsymbol{\alpha}_{g}^{\top}, \eta_{e}, \alpha_{e}\right)^{\top}$ é o vetor de parâmetros a ser estimado no espaço paramétrico

$$
\boldsymbol{\Theta}=\left\{\boldsymbol{\theta} \in \mathbb{R}^{s} \mid \boldsymbol{\beta} \in \mathbb{R}^{p}, \boldsymbol{\eta}_{g} \in \mathbb{R}^{+q}, \boldsymbol{\alpha}_{g} \in \mathbb{R}^{+(q+1)}, \eta_{e} \in \mathbb{R}^{+}, \alpha_{e} \in \mathbb{R}^{+}\right\}
$$

com $s=p+2 q+3$, e $\mathbb{R}^{+q}=\underbrace{\mathbb{R}^{+} \times \mathbb{R}^{+} \times \cdots \times \mathbb{R}^{+}}_{q \text { vezes }}$. A função de verossimilhança para o vetor $\mathbf{Y}=\left(\mathbf{Y}_{1}, \ldots, \mathbf{Y}_{n}\right)^{\top}$ é da forma

$$
L(\boldsymbol{\theta} ; \mathbf{Y})=\prod_{i=1}^{n} \int_{\mathbb{R}^{q}}\left\{\prod_{j=1}^{m_{i}} f_{Y \mid \boldsymbol{\gamma}}\left(y_{i j} \mid \boldsymbol{\gamma}_{i} ; \boldsymbol{\beta}, \eta_{e}, \alpha_{e}\right)\right\} f_{\boldsymbol{\gamma}}\left(\boldsymbol{\gamma}_{i} ; \boldsymbol{\eta}_{g}, \boldsymbol{\alpha}_{g}\right) d \boldsymbol{\gamma}_{i} .
$$

De novo a densidade marginal (4.18) e a função de verossimilhança (4.19) não têm expressões analíticas fechadas e utilizamos o método de integração numérica de Gauss-Hermite multivariado descrito na Seção 2.5.2 para a avaliação das integrais envolvidas na função de verossimilhança (4.4).

A integral $I_{i}$ na função de verossimilhança (4.19) é dada por

$$
\begin{aligned}
I_{i}=\int_{\mathbb{R}^{q}} & \left\{\prod_{j=1}^{m_{i}} f_{Y \mid \boldsymbol{\gamma}}\left(y_{i j} \mid \boldsymbol{\gamma}_{i} ; \boldsymbol{\beta}, \eta_{e}, \alpha_{e}\right)\right\} f_{\boldsymbol{\gamma}}\left(\boldsymbol{\gamma}_{i} ; \boldsymbol{\eta}_{g}, \boldsymbol{\alpha}_{g}\right) d \boldsymbol{\gamma}_{i} \\
=\int_{\mathbb{R}^{q}} & \left\{\prod_{j=1}^{m_{i}} f_{Y \mid \boldsymbol{\gamma}}\left(y_{i j} \mid \boldsymbol{\gamma}_{i} ; \boldsymbol{\beta}, \eta_{e}, \alpha_{e}\right)\right\} \frac{\Gamma(\alpha+q)}{\alpha^{q} \Gamma(\alpha)} \prod_{l=1}^{q} \frac{\alpha_{l}}{\eta_{l}} \phi\left(\frac{\gamma_{i l}}{\eta_{l}}\right)\left\{\Phi\left(\frac{\gamma_{i l}}{\eta_{l}}\right)\right\}^{-\frac{\alpha_{l}}{\alpha}-1} \\
& \times\left(\sum_{l=1}^{q}\left\{\Phi\left(\frac{\gamma_{i l}}{\eta_{l}}\right)\right\}^{-\frac{\alpha_{l}}{\alpha}}-(q-1)\right)^{-(\alpha+q)} d \boldsymbol{\gamma}_{i}
\end{aligned}
$$

e fazendo a mudança de variável $u_{i l}=\gamma_{i l} / \sqrt{2} \eta_{l}$, para $l=1, \ldots, q$, temos que a integral $I_{i}$ em 
(4.20) fica dada por:

$$
\begin{aligned}
I_{i}=\int_{\mathbb{R}^{q}} & \left\{\prod_{j=1}^{m_{i}} f_{Y \mid \boldsymbol{\gamma}}\left(y_{i j} \mid \mathbf{u}_{i}, \boldsymbol{\eta}_{g} ; \boldsymbol{\beta}, \eta_{e}, \alpha_{e}\right)\right\} \frac{\Gamma(\alpha+q)}{\alpha^{q} \Gamma(\alpha)} \prod_{l=1}^{q} \frac{\alpha_{l}}{\sqrt{\pi}}\left\{\Phi\left(\sqrt{2} u_{i l}\right)\right\}^{-\frac{\alpha_{l}}{\alpha}-1} \\
& \times\left(\sum_{l=1}^{q}\left\{\Phi\left(\sqrt{2} u_{i l}\right)\right\}^{-\frac{\alpha_{l}}{\alpha}}-(q-1)\right)^{-(\alpha+q)} \exp \left\{-\mathbf{u}_{i}^{\top} \mathbf{u}_{i}\right\} d \mathbf{u}_{i}
\end{aligned}
$$

em que $\mathbf{u}_{i}=\left(u_{i 1}, \ldots, u_{i q}\right)^{\top}$, e tem a forma (2.25). Segue que a função de verossimilhança (4.19) pode ser aproximada como:

$$
\begin{aligned}
L(\boldsymbol{\theta} ; \mathbf{Y}) \approx & \prod_{i=1}^{n}\left(\sum_{k_{1}=1}^{Q_{1}} \cdots \sum_{k_{q}=1}^{Q_{q}} \frac{\omega_{k_{1}} \cdots \omega_{k_{q}}}{\pi^{q / 2}} \prod_{j=1}^{m_{i}} f_{Y \mid \boldsymbol{\gamma}}\left(y_{i j} \mid \mathbf{u}_{k}, \boldsymbol{\eta}_{g} ; \boldsymbol{\beta}, \eta_{e}, \alpha_{e}\right) \frac{\Gamma(\alpha+q)}{\alpha^{q} \Gamma(\alpha)}\right. \\
& \left.\times \prod_{l=1}^{q} \alpha_{l}\left\{\Phi\left(\sqrt{2} u_{k_{l}}\right)\right\}^{-\frac{\alpha_{l}}{\alpha}-1}\left(\sum_{l=1}^{q}\left\{\Phi\left(\sqrt{2} u_{k_{l}}\right)\right\}^{-\frac{\alpha_{l}}{\alpha}}-(q-1)\right)^{-(\alpha+q)}\right)
\end{aligned}
$$

em que $\mathbf{u}_{k}=\left(u_{k_{1}}, \ldots, u_{k_{q}}\right)^{\top}$, e o logaritmo da função de verossimilhança $\ell(\boldsymbol{\theta} ; \mathbf{Y})$ pode ser escrito como:

$$
\begin{aligned}
\ell(\boldsymbol{\theta} ; \mathbf{Y}) \approx & \sum_{i=1}^{n} \log \left(\sum_{k_{1}=1}^{Q_{1}} \cdots \sum_{k_{q}=1}^{Q_{q}} \frac{\omega_{k_{1}} \cdots \omega_{k_{q}}}{\pi^{q / 2}} \prod_{j=1}^{m_{i}} f_{Y \mid \boldsymbol{\gamma}}\left(y_{i j} \mid \mathbf{u}_{k}, \boldsymbol{\eta}_{g} ; \boldsymbol{\beta}, \eta_{e}, \alpha_{e}\right) \frac{\Gamma(\alpha+q)}{\alpha^{q} \Gamma(\alpha)}\right. \\
& \left.\times \prod_{l=1}^{q} \alpha_{l}\left\{\Phi\left(\sqrt{2} u_{k_{l}}\right)\right\}^{-\frac{\alpha_{l}}{\alpha}-1}\left(\sum_{l=1}^{q}\left\{\Phi\left(\sqrt{2} u_{k_{l}}\right)\right\}^{-\frac{\alpha_{l}}{\alpha}}-(q-1)\right)^{-(\alpha+q)}\right)
\end{aligned}
$$

A função escore aproximada é obtida diferenciando o logaritmo da função deverossimilhança $\ell(\boldsymbol{\theta} ; \mathbf{Y})$ em relação a cada um dos parâmetros e é dada por

$$
\boldsymbol{U}(\boldsymbol{\theta})=\left(\boldsymbol{U}_{\boldsymbol{\beta}}^{\top}(\boldsymbol{\theta}), \boldsymbol{U}_{\boldsymbol{\eta}_{g}}^{\top}(\boldsymbol{\theta}), \boldsymbol{U}_{\boldsymbol{\alpha}_{g}}^{\top}(\boldsymbol{\theta}), U_{\eta_{e}}(\boldsymbol{\theta}), U_{\alpha_{e}}(\boldsymbol{\theta})\right)^{\top}
$$

No Apêndice B.5.3 são apresentadas as expressões para os componentes do vetor $\boldsymbol{U}(\boldsymbol{\theta})$. O estimador de máxima verossimilhança de $\boldsymbol{\theta}=\left(\boldsymbol{\beta}^{\top}, \boldsymbol{\eta}_{g}^{\top}, \boldsymbol{\alpha}_{g}^{\top}, \eta_{e}, \alpha_{e}\right)^{\top}$ é obtido como solução ao sistema de equações $\boldsymbol{U}(\boldsymbol{\theta})=\mathbf{0}$. Sob as condições usuais de regularidade, o estimador de máxima verossimilhança $\widehat{\boldsymbol{\theta}}$, e assintóticamente normal com média $\boldsymbol{\theta}$ e matriz de covariâncias $\boldsymbol{J}^{-1}(\boldsymbol{\theta})$, isto é,

$$
\sqrt{n}(\widehat{\boldsymbol{\theta}}-\boldsymbol{\theta}) \rightarrow N\left(\mathbf{0}, \boldsymbol{J}^{-1}(\boldsymbol{\theta})\right)
$$

em que $\boldsymbol{J}(\boldsymbol{\theta})$ é a matriz de informação observada, obtida como $\boldsymbol{J}(\boldsymbol{\theta})=-\partial^{2} \ell(\boldsymbol{\theta} ; \mathbf{Y}) / \partial \boldsymbol{\theta} \partial \boldsymbol{\theta}^{\top}$.

\subsection{Simulação}

Com o intuito de estudar as propriedades dos estimadores de máxima verossimilhança $\widehat{\boldsymbol{\beta}}, \widehat{\eta}_{e}$, $\widehat{\alpha}_{e}, \widehat{\eta}_{b}$ e $\widehat{\alpha}_{g}$, de $\boldsymbol{\beta}, \eta_{e}, \alpha_{e}, \eta_{g}$ e $\alpha_{g}$, respectivamente; realizamos um estudo de simulação de Monte Carlo, em que foi considerado o modelo linear com interceptos aleatórios

$$
Y_{i j}=\beta_{0}+\beta_{1} x_{i j}+\gamma_{i}+\epsilon_{i j}
$$


$\operatorname{com} \gamma_{i} \stackrel{\mathrm{iid}}{\sim} \mathrm{PN}\left(0, \eta_{g}, \alpha_{g}\right)$ e $\epsilon_{i j} \stackrel{\mathrm{iid}}{\sim} \mathrm{PN}\left(0, \eta_{e}, \alpha_{e}\right)$, para $j=1, \ldots, 5$ e $i=1, \ldots, n$. Os verdadeiros valores dos parâmetros foram tomados como $\beta_{0}=2, \beta_{1}=1.5, \eta_{e}=\eta_{g}=0.50, \alpha_{e}=0.50,1.5$ e $\alpha_{g}=0.50,1.50,3.0$. Nós tomamos esses valores de $\alpha$ para acomodar assimetria à esquerda (valores de $\alpha_{e}$ e $\alpha_{g}$ menores que 1) e à direita (valores de $\alpha_{e}$ e $\alpha_{g}$ maiores que 1). A covariável $x_{i}$ foi gerada de acordo a uma distribuição $\mathrm{U}(0.1,20)$. Foram simulados 1000 conjuntos da datos de Monte Carlo de tamanhos $n=50,100,200,400$ e 700 do modelo (4.25).

Para estudar as propriedades assintóticas dos estimadores, consideramos o viés relativo (VR) em valor absoluto, e a raiz quadrada do erro quadrático médio (REQM) os quais são dados por

$$
\operatorname{VR}\left(\widehat{\theta}_{i}\right)=\frac{1}{1000} \sum_{k=1}^{1000}\left(\frac{\widehat{\theta}_{i}^{(k)}}{\theta_{i}}-1\right), \operatorname{REQM}\left(\widehat{\theta}_{i}\right)=\sqrt{\frac{1}{1000} \sum_{k=1}^{1000}\left(\widehat{\theta}_{i}^{(k)}-\theta_{i}\right)^{2}},
$$

respectivamente, em que $\widehat{\theta}_{i}^{(k)}$ é o estimador de $\theta_{i}$ para a $k$-ésima amostra, para $\theta_{i} \in \boldsymbol{\theta}=$ $\left(\beta_{0}, \beta_{1}, \eta_{e}, \eta_{g}, \alpha_{e}, \alpha_{g}\right)^{\top}$. Os estimadores de máxima verossimilhança dos parâmetros foram computados usando a função optim do pacote estatístico R Development Core Team (2017), maximizando a função de logverossimilhança em (4.7). Em todas as simulações foram usados 15 pontos na quadratura de Gaus-Hermite para aproximar as integrais envolvidas na função de log-verosimilhança.

Da Tabela A.2 no Apêndice A pode-se observar que os estimadores dos parâmetros são estáveis. Também o VR e a REQM dos estimadores tendem se aproximar de zero quando o valor de $n$ aumenta indicando que os estimadores baseados no método de máxima verossimilhança fornecem boas propriedades assintóticas. Esse padrão é o mesmo para os diferentes valores de $\alpha_{e}$ e $\alpha_{g}$ sob consideração. Concluímos que os estimadores de máxima verossimilhança funcionam bastante bem quando o tamanho das amostras é maior ou igual a 200.

\subsection{Aplicação}

Nesta seção aplicamos o modelo proposto a um conjunto de dados longitudinais coletados como parte do estudo de colesterol em Framingham (Framingham cholesterol study). O conjunto de dados consiste de 200 indivíduos aleatoriamente selecionados e inclui os níveis de colesterol em diferentes instantes de tempo, sexo e a idade no início do estudo (baseline). O conjunto de dados é reportado em Zhang e Davidiam (2001) em que foi mostrado mediante um estudo informal que o comportamento assimétrico é parcialmente explicado pelas covariáveis apresentadas e que os efeitos aleatórios podem não ser normalmente distribuídos.

A Tabela 4.1 apresenta os coeficientes de assimetria e curtose para os níveis do colesterol. Note-se que os valores dos coeficientes justificam o uso de um modelo assimétrico (modelo normalpotência, por exemplo). Além disso, o histograma dos níveis de colesterol dado na Figura 4.1 mostra claramente a presença de assimetria e parece adequado considerar um modelo potêncianormal para analisar o conjunto de dados. Figura 4.1 também retrata as trajetórias de cinco indivíduos selecionados aleatoriamente. Este conjunto de dados também foi analisado anteriormente por Arellano-Valle et al. (2005) usando um modelo linear misto com efeitos aleatórios distribuidos normal-assimétrico.

O seguinte modelo linear misto foi adotado:

$$
Y_{i j}=\beta_{0}+\beta_{1} \operatorname{sexo}_{i}+\beta_{2} \operatorname{idade}_{i}+\beta_{3} t_{i j}+\gamma_{i}+\epsilon_{i j}
$$

em que $Y_{i j}$ é o nível de colesterol $(\mathrm{mg} / \mathrm{dL})$ avaliado no $j$-ésimo tempo $\left(j=1, \ldots, m_{i}\right)$ para o sujeito $i(i=1, \ldots, 200)$, sexo $_{i}$ é o indicador do sexo do sujeito $(0=$ feminino, $1=$ masculino $)$; 
Tabela 4.1: Resumo estatístico para os dados de colesterol.

\begin{tabular}{ccccc}
\hline$n$ & Média & Erro padrão & Assimetria & Curtose \\
\hline 200 & 2.342 & 0.4595 & 0.6865 & 3.4423 \\
\hline
\end{tabular}
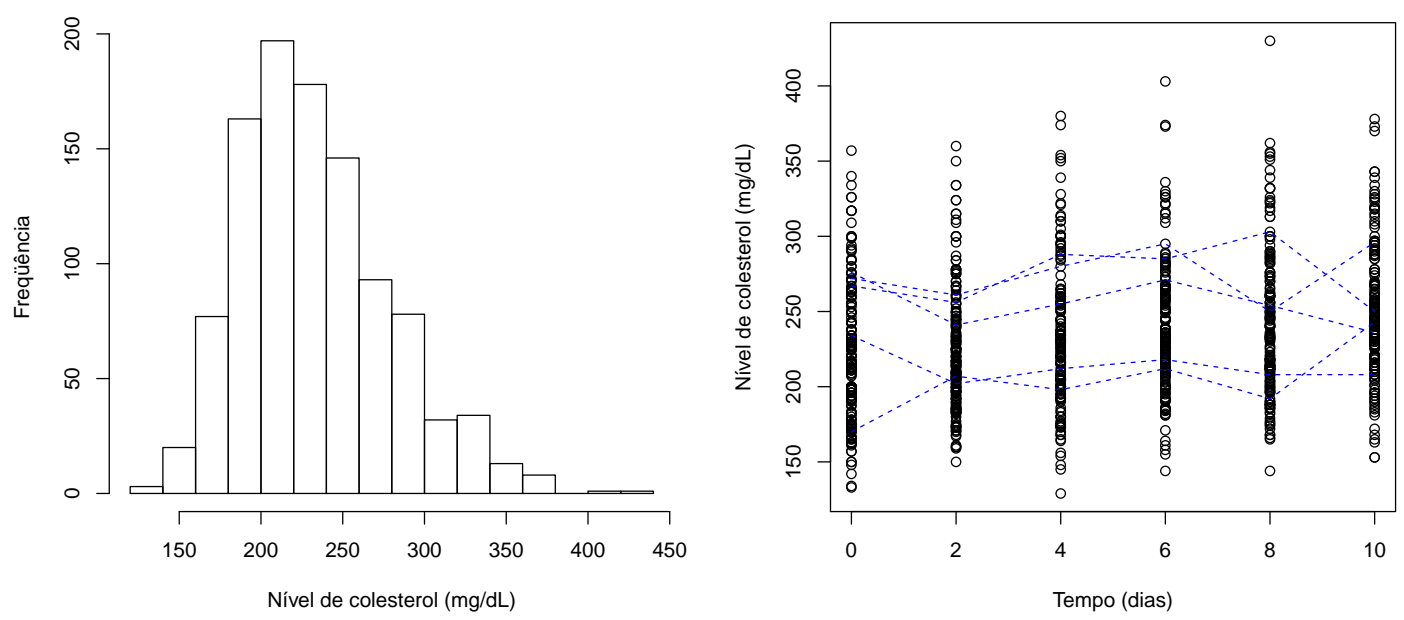

Figura 4.1: Dados de colesterol: (a) Histograma do nível de colesterol para 200 sujeitos do estudo de Framingham. (b) Trajetórias para cinco sujeitos aleatóriamente selecionados.

$i d a d e_{i}$ é a idade no início do estudo (em anos); e $t_{i j}$ é (tempo - 5)/10, com tempo medido em anos.

Baseados nas considerações acima, os seguintes quatro modelos estatísticos, os quais diferem na distribuiçao dos erros e os efeitos aleatórios, foram analisados

- Um modelo com distribuição normal-potência para os erros e os efeitos aleatórios, isto é, $\epsilon_{i j} \stackrel{\text { iid }}{\sim} \mathrm{PN}\left(0, \eta_{e}, \alpha_{e}\right)$ e $\gamma_{i} \stackrel{\text { iid }}{\sim} \mathrm{PN}\left(0, \eta_{g}, \alpha_{g}\right)$; e que chamaremos (modelo $\left.P N-P N\right)$,

- Um modelo com distribuição normal para os erros e distribuição normal-potência para os efeitos aleatórios, isto é, $\epsilon_{i j} \stackrel{\text { iid }}{\sim} \mathrm{N}\left(0, \eta_{e}^{2}\right)$ e $\gamma_{i} \stackrel{\text { iid }}{\sim} \mathrm{PN}\left(0, \eta_{g}, \alpha_{g}\right)$; e que chamaremos (modelo $N-P N)$,

- Um modelo com distribuição normal para os erros e distribuição normal-assimétrico para os efeitos aleatórios, isto é, $\epsilon_{i j} \stackrel{\mathrm{iid}}{\sim} \mathrm{N}\left(0, \eta_{e}^{2}\right)$ e $\gamma_{i} \stackrel{\mathrm{iid}}{\sim} \mathrm{SN}\left(0, \eta_{g}, \lambda_{g}\right)$; e que chamaremos (modelo $N-S N)$,

- Um modelo com distribuição normal para os erros e os efeitos aleatórios, isto é, $\epsilon_{i j} \stackrel{\text { iid }}{\sim}$ $\mathrm{N}\left(0, \eta_{e}^{2}\right)$ e $\gamma_{i} \stackrel{\text { iid }}{\sim} \mathrm{N}\left(0, \eta_{g}^{2}\right)$; e que chamaremos (modelo $\left.N-N\right)$.

De nossa análise, resultou que a covariável sexo não foi significativa para os modelos considerados e portanto, não foi incluída. As estimativas dos parâmetros (apenas para as variáveis importantes), as quais foram obtidos usando a função optim no R (R Development Core Team , 2017), são mostradas na Tabela 4.2. As estimativas dos parâmetros $\mu_{g}$ e $\mu_{e}$ e seus respectivos 
Tabela 4.2: Estimativas de MV dos parâmetros e erros padrões (EP) obtidos no ajuste dos modelos PNPN, NPN, NSN, e N para os dados de colesterol.

\begin{tabular}{|c|c|c|c|c|c|c|c|c|}
\hline \multirow[b]{2}{*}{$\theta$} & \multicolumn{2}{|c|}{ Modelo PN-PN } & \multicolumn{2}{|c|}{ Modelo N-PN } & \multicolumn{2}{|c|}{ Modelo N-SN } & \multicolumn{2}{|c|}{ Modelo N-N } \\
\hline & Estimativa & EP & Estimativa & $\mathrm{EP}$ & Estimativa & EP & Estimativa & EP \\
\hline$\beta_{0}$ & 120.348 & 15.454 & 138.777 & 9.898 & 128.179 & 10.089 & 167.637 & 14.793 \\
\hline$\beta_{2}$ & 1.023 & 0.239 & 1.083 & 0.237 & 1.352 & 0.246 & 1.570 & 0.323 \\
\hline$\beta_{3}$ & 28.569 & 2.013 & 28.258 & 2.025 & 27.951 & 2.056 & 28.244 & 2.021 \\
\hline$\eta_{g}$ & 50.348 & 1.942 & 51.114 & 1.922 & 55.875 & 2.329 & 37.189 & 2.027 \\
\hline$\eta_{e}$ & 28.698 & 3.738 & 22.074 & 0.524 & 22.402 & 0.531 & 22.048 & 0.536 \\
\hline$\alpha_{g}$ & 3.677 & 0.332 & 3.699 & 0.325 & - & - & - & - \\
\hline$\alpha_{e}$ & 2.655 & 1.377 & - & - & - & - & - & - \\
\hline$\lambda_{g}$ & - & - & - & - & 3.459 & 0.698 & - & - \\
\hline$\mu_{g}$ & 49.125 & 0.051 & 50.157 & 0.052 & - & - & - & - \\
\hline$\mu_{e}$ & 21.961 & 0.031 & - & - & - & - & - & - \\
\hline
\end{tabular}

Tabela 4.3: Critérios de seleção dos modelos ajustados aos dados de colesterol.

\begin{tabular}{lcccc}
\hline Critério & Modelo PN-PN & Modelo N-PN & Modelo N-SN & Modelo N-N \\
\hline$\ell(\boldsymbol{\theta})$ & -4973.9 & -4976.2 & -4979.7 & -4982.0 \\
mAIC & 9961.9 & 9964.5 & 9971.5 & 9974.1 \\
BIC $_{h}$ & 9993.2 & 9990.8 & 9997.8 & 9997.1 \\
CAIC & 9996.5 & 9994.1 & 10001.1 & 9998.8 \\
HQIC & 9961.4 & 9964.0 & 9971.0 & 9973.7 \\
\hline
\end{tabular}

erros padrão aproximados também mostrados na Tabela 4.2 foram obtidas usando integração numérica de Monte Carlo utilizando as estimativas de máxima verossimilhança de $\widehat{\alpha}_{g}, \widehat{\alpha}_{e}, \widehat{\eta}_{g}$ e $\widehat{\eta}_{e}$.

Para comparar o ajuste dos modelos, avaliamos uma série de ajustes por inspeção de critérios de informação apresentados na Seção 4.2.4 e que são mostrados na Tabela 4.3. De acordo com os critérios $\mathrm{BIC}_{h}$ e CAIC, o modelo NPN parece proporcionar um melhor ajuste aos dados de colesterol do que os outros modelos considerados, apoiando a afirmação de afastamento da normalidade do efeito aleatório. Também podemos ver a partir da Tabela 4.3 que de acordo com os critérios mAIC e HQIC, o modelo PN-PN é melhor do que o modelo N-PN, e todos os critérios indicam que o modelo PN-PN parece proporcionar um ajuste melhor do que o modelo N-SN e o modelo normal. Isso também pode ser observado comparando o gráfico de probabilidade meionormal com envelope simulado para o resíduo Pearson (resíduo marginal padronizado) na Figura 4.2 .

A suposição de distribuição normal-potência para os erros e os interceptos aleatórios é testada com a hipótese

$$
H_{01}:\left(\alpha_{g}, \alpha_{e}\right)=(1,1) \text { versus } H_{11}:\left(\alpha_{g}, \alpha_{e}\right) \neq(1,1),
$$

usando a estatística de razão de veossimilhança (RV)

$$
\Lambda_{1}=\frac{L_{N-N}(\widehat{\boldsymbol{\theta}})}{L_{P N-P N}(\widehat{\boldsymbol{\theta}})},
$$

a qual sob os dados em consideração, leva a $-2 \log \left(\Lambda_{1}\right)=-2\left(\ell_{N-N}(\widehat{\boldsymbol{\theta}})-\ell_{P N-P N}(\widehat{\boldsymbol{\theta}})\right)=16.10$, com $p$-value $<0.05$, com forte indicação contra a hipótese nula. 


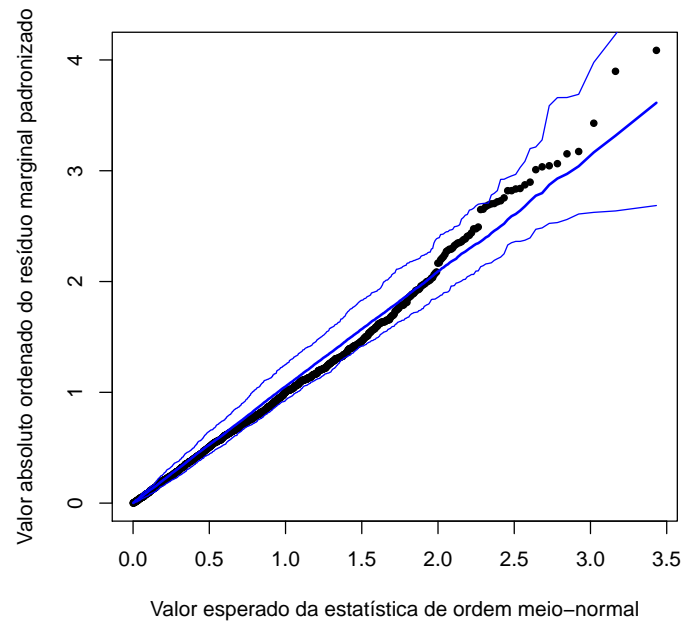

(a)

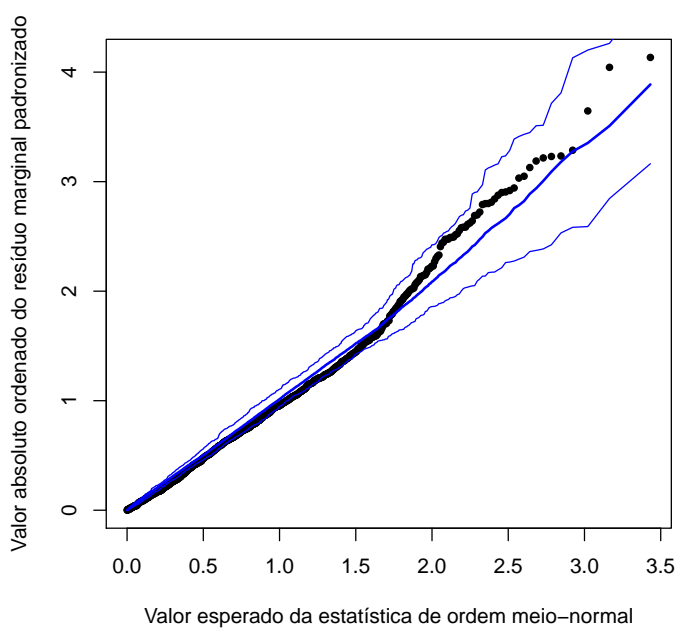

(c)

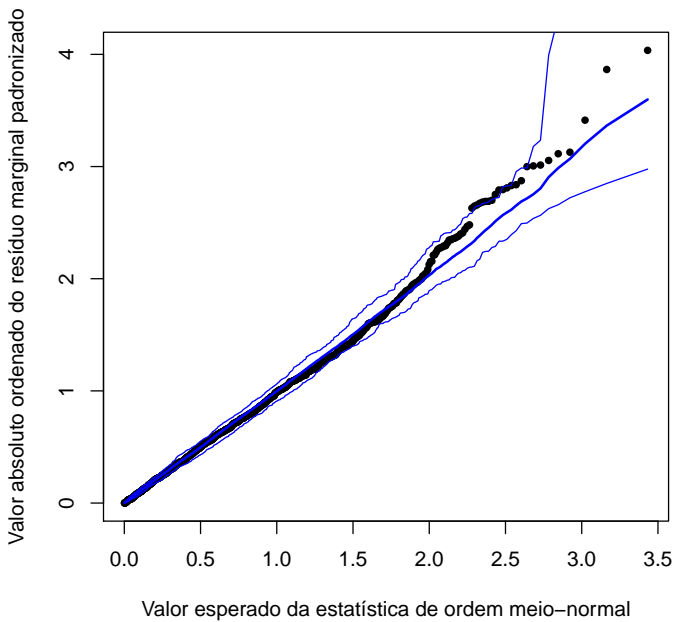

(b)

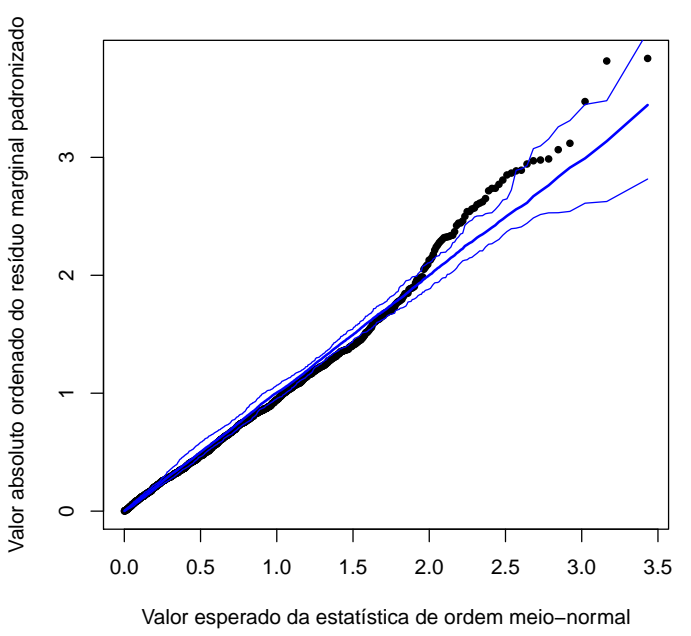

(d)

Figura 4.2: Gráfico de probabilidade meio-normal com envelope simulado para o modelo PN-PN (a), modelo N-PN (b), modelo N-SN (c), e modelo N-N (d).

Similarmente, a suposição de distribuição normal-potência para os efeitos aleatórios, e normalidade dos erros é testada usando a hipótese

$$
H_{02}: \alpha_{g}=1 \quad \text { versus } \quad H_{12}: \alpha_{g} \neq 1,
$$

usando a estatística de RV

$$
\Lambda_{2}=\frac{L_{N-P N}(\widehat{\boldsymbol{\theta}})}{L_{P N-P N}(\widehat{\boldsymbol{\theta}})},
$$

que para os dados sob estudo leva a $-2 \log \left(\Lambda_{2}\right)=-2\left(\ell_{N-P N}(\widehat{\boldsymbol{\theta}})-\ell_{P N-P N}(\widehat{\boldsymbol{\theta}})\right)=5.04$, com $p-$ value $=0.0248$, com forte indicação em contra a hipótese nula. Portanto, o modelo N-PN 
ajusta os dados de colesterol melhor do que o modelo linear com erros e interceptos aleatórios normalmente distribuídos, e o modelo PN-PN ajusta melhor os dados do que o modelo N-PN.

\subsection{Conclusões}

Neste capítulo, abordamos o problema de modelar a relação entre uma variável resposta com coeficiente de assimetria e curtose maior ou menor do que pode ser modelado pela distribuição normal; e um conjunto de variáveis explicativas. Propomos a distribuição normal-potência para dar flexibilidade ao termo do erro e ao efeito aleatório em um modelo linear misto.

A vantagem de considerar essa distribuição é que pode oferecer flexibilidade na captura de uma ampla gama de comportamentos não normais, incluindo formas assimétricas à esquerda e à direita, tendo a normalidade como caso especial. Derivamos os dois primeiros momentos da variável resposta, e propomos um método de estimação dos parâmetros do modelo, bem como uma metodologia para obter as predições dos interceptos aleatórios. A predição dos efeitos aleatórios foi realizada considerando a metodologia do melhor preditor de Bayes empírico. Derivamos também a função escore aproximada e os elementos aproximados da matriz de informação observada.

A análise residual também foi apresentada e as estimativas de parâmetros são obtidas usando a abordagen de máxima verossimilhança com métodos de integração numérica (quadratura de Gauss-Hermite) que também são usados para estudar propriedades assintóticas dos estimadores. Um estudo de simulação em pequena escala indica boa recuperação de parâmetros com o método de estimatição desenvolvido. Além disso, apresentamos um exemplo de motivação para ilustrar a nossa proposta e concluímos que o modelo linear misto normal-potência pode ser uma alternativa útil a outros métodos existentes na literatura como o modelo linear misto normal-assimétrico (Skew-normal linear mixed model, SNLMM) por Arellano-Valle et al. (2005). Então, acreditamos que o modelo proposto neste capítulo amplia a opção na classe de modelos lineares mistos, para ajustar dados particularmente assimétricos com indicação de distribuição não normal para o efeito aleatório. 


\section{Capítulo 5}

\section{Modelos de Regressão Lineares Mistos Assimétricos para Dados Duplamente Censurados}

\subsection{Introdução}

Uma variável aleatória é dita ser duplamente censurada, quando as medições acima do limite de deteção máximo e abaixo do limite de deteção minimo, são tomadas como esses valores. Os limites de deteção mínimo e máximo são especificados pelo pesquisador e geralmente dependem do dispositivo de medição utilizado para produzir as medições. Por exemplo, nos dados sobre vacina analisados em Moulton e Hasley (1995) (dados censurados à esquerda), o limite de deteção mínimo foi tomado como 0,1 U.N. (unidades internacionais). Um cenário com resposta duplamente censurada é considerado em Galvis et al. (2014), em que o conjunto de dados é relacionado a um estudo clínico, em que a resposta de interesse é a proporção de locais doentes de um tipo específico de dente. Neste conjunto de dados, temos a situação de aumento de zeros e uns.

O modelo tobit usual (Tobin, 1958), algumas vezes usado em situações como as expostas acima, pode não ser adequado para modelar dados censurados em que os valores observados para a parte contínua, apresentam assimetria e curtose maior ou menor do que é esperado para o modelo normal; em tais situações, o modelo normal-potência pode ser uma alternativa viável.

Martínez-Flórez et al. (2015) introduzem o modelo normal-potência duplamente censurado como segue: suponhamos que $Y^{*} \sim \operatorname{PN}(\xi, \eta, \alpha)$ e considere $\left(y_{1}^{*}, \ldots, y_{n}^{*}\right)$, uma amostra aleatória de tamanho $n$ de $Y^{*}$, e que parte dos $y^{*}$ estão entre as constantes $c_{0}$ e $c_{2}$, com $c_{0}<c_{2}$. Para $y^{*}<c_{0}$, somente $c_{0}$ é observado e para $y>c_{2}$ somente $c_{2}$ é observado.

\subsection{Modelo de Regressão Duplamente Censurado com Efeitos Aleatórios}

Consideremos o modelo de regressão linear dado por

$$
Y_{i j}=\mathbf{x}_{i j}^{\top} \boldsymbol{\beta}+\gamma_{i}+\epsilon_{i j},
$$

em que $Y_{i j}$ é o valor da $j$-ésima variável resposta para a $i$-ésima unidade amostral (sujeito), para $i=1, \ldots, n$, e $j=1, \ldots, m_{i} ; \mathbf{x}_{i j}=\left(x_{i j 1}, \ldots, x_{i j p}\right)^{\top}$ é um vetor conhecido de dimensão $p \times 1$ de variáveis explicativas associadas aos efeitos fixos para $i=1, \ldots, n$ e $j=1, \ldots, m_{i}$; 
$\boldsymbol{\beta}=\left(\beta_{1}, \ldots, \beta_{p}\right)^{\top}$ é um vetor de parâmetros desconhecido de dimensão $p \times 1$; os $\gamma_{i}$ 's são variáveis aleatórias independentes e igualmente distribuídas $f_{\gamma}\left(\gamma_{i} ; \boldsymbol{\theta}_{\gamma}\right)$, associadas à $i$-ésima unidade amostral; e os $\epsilon_{i j}$ 's são também variáveis aleatórias independentes e igualmente distribuídas $f_{\epsilon}\left(\epsilon_{i j} ; \boldsymbol{\theta}_{\epsilon}\right)$, (independentes de $\gamma_{i}$ ).

Vamos supor que para uma amostra aleatória de vetores $\mathbf{Y}_{1}, \ldots, \mathbf{Y}_{n}$ de tamnaho $n$ em que para $\mathbf{Y}_{i}=\left(Y_{i 1}, \ldots, Y_{i m_{i}}\right)^{\top}$, parte dos $Y_{i j}$ estão entre as constantes $c_{0 i j}$ e $c_{2 i j} \operatorname{com} c_{0 i j}<c_{2 i j}$. Temos que para valores $Y_{i j} \leq c_{0 i j}$ somente o valor $c_{0 i j}$ é relatado, enquanto que para valores $Y_{i j} \geq c_{2 i j}$ somente o valor $c_{2 i j}$ é relatado. Portanto os valores observados $Y_{i j}^{o}$, podem ser escritos como

$$
Y_{i j}^{o}= \begin{cases}c_{0 i j}, & \text { se } Y_{i j} \leq c_{0 i j}, \\ Y_{i j}, & \text { se } c_{0 i j}<Y_{i j}<c_{2 i j}, \\ c_{2 i j}, & \text { se } Y_{i j} \geq c_{2 i j} .\end{cases}
$$

para $i=1, \ldots, n$, e $j=1, \ldots, m_{i}$. A amostra resultante é dita ser uma amostra duplamente censurada. Para as observações $Y_{i j}^{o}=c_{0 i j}$, temos que

$$
\mathrm{P}\left(Y_{i j}^{o}=c_{0 i j}\right)=\mathrm{P}\left(Y_{i j} \leq c_{0 i j}\right)=p_{0 i j}
$$

enquanto que para $Y_{i j}^{o}=c_{2 i j}$, temos que

$$
\mathrm{P}\left(Y_{i j}^{o}=c_{2 i j}\right)=\mathrm{P}\left(Y_{i j} \geq c_{2 i j}\right)=p_{2 i j}
$$

Finalmente, para respostas contínuas, isto é, $c_{0 i j}<Y_{i j}<c_{2 i j}$ vamos definir $\mathrm{P}\left(c_{0 i j}<Y_{i j}<\right.$ $\left.c_{2 i j}\right)=p_{1 i j}$. Note-se que as probabilidades $p_{0 i j}, p_{1 i j}$ e $p_{2 i j}$ dependem das distribuições assumidas para as variáveis aleatórias $\gamma_{i}$ e $\epsilon_{i j}$, e a $i j$-ésima resposta observada pode ser escrita como

$$
Y_{i j}^{o}=D_{0 i j} c_{0 i j}+D_{2 i j} c_{2 i j}+\left(1-D_{0 i j}-D_{2 i j}\right) Y_{i j}
$$

em que $D_{0 i j}=I\left(Y_{i j} \leq c_{0 i j}\right), D_{2 i j}=I\left(Y_{i j} \geq c_{2 i j}\right)$ e $Y_{i j}=\mathbf{x}_{i j}^{\top} \boldsymbol{\beta}+\gamma_{i}+\epsilon_{i j} \operatorname{com} \gamma_{i} \stackrel{\text { iid }}{\sim} f_{\gamma}\left(\gamma_{i} ; \boldsymbol{\theta}_{\gamma}\right)$ e $\epsilon_{i j} \stackrel{\mathrm{iid}}{\sim} f_{\epsilon}\left(\epsilon_{i j} ; \boldsymbol{\theta}_{\epsilon}\right), i=1, \ldots, n$, e $j=1, \ldots, m_{i}$.

Dada a função densidade de probabilidade (fdp) $f_{\epsilon}\left(\epsilon_{i j} ; \boldsymbol{\theta}_{\epsilon}\right)$, denotamos por $f_{Y \mid \gamma}\left(y_{i j} \mid \gamma_{i} ; \boldsymbol{\beta}, \boldsymbol{\theta}_{\epsilon}\right)$ a fdp de $Y_{i j} \mid \gamma_{i}$ e considerando $f_{\gamma}\left(\gamma_{i} ; \boldsymbol{\theta}_{\gamma}\right)$, se segue que a fdp marginal do vetor aleatório observado $\mathbf{Y}_{i}^{o}=\left(Y_{i 1}^{o}, \ldots, Y_{i m_{i}}^{o}\right)^{\top}$ é dado por

$$
\begin{aligned}
f\left(\mathbf{y}_{i}^{o} ; \boldsymbol{\theta}\right)=\int_{\mathbb{R}}\left\{\prod_{j=1}^{m_{i}}\left[\mathrm{P}\left(Y_{i j}^{o}=c_{0 i j}\right)\right]^{d_{0 i j}}\left[\mathrm{P}\left(Y_{i j}^{o}=c_{2 i j}\right)\right]^{d_{2 i j}}\right. \\
\left.\times\left[f_{Y}\left(y_{i j} \mid \gamma_{i} ; \boldsymbol{\beta}, \boldsymbol{\theta}_{\epsilon}\right)\right]^{1-d_{0 i j}-d_{2 i j}}\right\} f_{\gamma}\left(\gamma_{i} ; \boldsymbol{\theta}_{\gamma}\right) d \gamma_{i},
\end{aligned}
$$

em que

$$
d_{0 i j}=\left\{\begin{array}{ll}
1 & \text { se } Y_{i j}^{o}=c_{0 i j}, \\
0 & \text { caso contrário. }
\end{array} \quad d_{2 i j}= \begin{cases}1 & \text { se } Y_{i j}^{o}=c_{2 i j}, \\
0 & \text { caso contrário. }\end{cases}\right.
$$

A fdp marginal em (5.3) é composta pela contribuição de três partes, duas correspondentes aos valores que a variável aleatória $Y_{i j}$ é censurada, e uma parte em que a variável aleatória $Y_{i j}$ pode ser observada.

O modelo descrito acima, nós chamaremos de modelo de regressão duplamente censurados com efeitos aleatórios e será denotado por DCRM-RE (Doubly Censored Regression Model with Random Effects) 


\subsubsection{Momentos}

Os momentos no modelo DCRM-RE, podem ser calculados seguindo o mesmo procedimento que nos modelos lineares mistos normal-potência no Capítulo 3. Usando a abordagem marginal para modelos mistos descrita na Seção 2.2.1 junto com a equação (5.2) é possível calcular a média e a variância de $Y_{i j}^{o}$; e a covariáncia e correlação entre as observaçoes $Y_{i j}^{o}$ e $Y_{i j^{\prime}}^{o}$.

\section{Média}

Definindo $\epsilon_{0 i j}=c_{0 i j}-\mathbf{x}_{i j}^{\top} \boldsymbol{\beta}-\gamma_{i}$ e $\epsilon_{2 i j}=c_{2 i j}-\mathbf{x}_{i j}^{\top} \boldsymbol{\beta}-\gamma_{i}$, se segue que a média da observação $Y_{i j}^{o}$ é dada por

$$
\begin{aligned}
\mathrm{E}\left[Y_{i j}^{o}\right]= & c_{0 i j} \mathrm{P}\left(Y_{i j} \leq c_{0 i j}\right)+c_{2 i j} \mathrm{P}\left(Y_{i j} \geq c_{2 i j}\right) \\
& \quad+\mathrm{E}\left[Y_{i j} \mid c_{0 i j}<Y_{i j}<c_{2 i j}\right] \mathrm{P}\left(c_{0 i j}<Y_{i j}<c_{2 i j}\right) \\
= & c_{0 i j} p_{0 i j}+c_{2 i j} p_{2 i j}+\left(\mathbf{x}_{i j}^{\top} \boldsymbol{\beta}+\mathrm{E}\left[\gamma_{i}\right]+\mathrm{E}\left[\epsilon_{i j} \mid \epsilon_{0 i j}<\epsilon_{i j}<\epsilon_{2 i j}\right]\right) p_{1 i j} .
\end{aligned}
$$

\section{Variáncia}

A variância da observação $Y_{i j}^{o}$ pode ser calculada como

$$
\begin{aligned}
\operatorname{Var}\left[Y_{i j}^{o}\right]= & c_{0 i j}^{2} \mathrm{P}\left(Y_{i j} \leq c_{0 i j}\right)+c_{2 i j}^{2} \mathrm{P}\left(Y_{i j} \geq c_{2 i j}\right) \\
& +\mathrm{E}\left[Y_{i j}^{2} \mid c_{0 i j}<Y_{i j}<c_{2 i j}\right] \mathrm{P}\left(c_{0 i j}<Y_{i j}<c_{2 i j}\right)-\left(E\left[Y_{i j}^{o}\right]\right)^{2} \\
= & c_{0 i j}^{2} p_{0 i j}\left(1-p_{0 i j}\right)+c_{2 i j}^{2} p_{2 i j}\left(1-p_{2 i j}\right)-2 c_{0 i j} c_{2 i j} p_{0 i j} p_{2 i j} \\
& +\left\{\operatorname{Var}\left[\gamma_{i}\right]+\operatorname{Var}\left[\epsilon_{i j} \mid \epsilon_{0 i j}<\epsilon_{i j}<\epsilon_{2 i j}\right]\right. \\
& +\left(1-p_{1 i j}\right)\left(\mathbf{x}_{i j}^{\top} \boldsymbol{\beta}+\mathrm{E}\left[\gamma_{i}\right]+\mathrm{E}\left[\epsilon_{i j} \mid \epsilon_{0 i j}<\epsilon_{i j}<\epsilon_{2 i j}\right]\right)^{2} \\
& \left.-2\left(c_{0 i j} p_{0 i j}+c_{2 i j} p_{2 i j}\right)\left(\mathbf{x}_{i j}^{\top} \boldsymbol{\beta}+\mathrm{E}\left[\gamma_{i}\right]+\mathrm{E}\left[\epsilon_{i j} \mid \epsilon_{0 i j}<\epsilon_{i j}<\epsilon_{2 i j}\right]\right)\right\} p_{1 i j} .
\end{aligned}
$$

\section{Covariância e Correlação}

Assumindo independência condicional dos $Y_{i j}^{o}$, temos que a covariância entre $Y_{i j}^{o}$ e $Y_{i j^{\prime}}^{o}$, duas observações nos momentos $j$ e $j^{\prime}\left(j \neq j^{\prime}\right)$ do $i$-ésimo indivíduo, é dada por

$$
\begin{aligned}
\operatorname{Cov}\left[Y_{i j}^{o}, Y_{i j^{\prime}}^{o}\right]= & \mathrm{E}\left[Y_{i j}^{o} Y_{i j^{\prime}}^{o}\right]-\mathrm{E}\left[Y_{i j}^{o}\right] \mathrm{E}\left[Y_{i j^{\prime}}^{o}\right] \\
= & \mathrm{E}\left[Y_{i j} Y_{i j^{\prime}} \mid c_{0 i j}<Y_{i j}<c_{2 i j} ; c_{0 i j^{\prime}}<Y_{i j^{\prime}}<c_{2 i j^{\prime}}\right] p_{1 i j} p_{1 i j^{\prime}} \\
& \quad-\mathrm{E}\left[Y_{i j} \mid c_{0 i j}<Y_{i j}<c_{2 i j}\right] \mathrm{E}\left[Y_{i j^{\prime}} \mid c_{0 i j^{\prime}}<Y_{i j^{\prime}}<c_{2 i j^{\prime}}\right] p_{1 i j} p_{1 i j^{\prime}} \\
= & p_{1 i j} p_{1 i j^{\prime}} \operatorname{Var}\left[\gamma_{i}\right] .
\end{aligned}
$$

Segue de (5.5) e (5.6) que a correlação entre $Y_{i j}$ e $Y_{i j^{\prime}}$, duas observações nos momentos $j$ e $j^{\prime}$ $\left(j \neq j^{\prime}\right)$ do $i$-ésimo indivíduo, é dada por

$$
\operatorname{Corr}\left[Y_{i j}^{o}, Y_{i j^{\prime}}^{o}\right]=\frac{p_{1 i j} p_{1 i j^{\prime}} \operatorname{Var}\left[\gamma_{i}\right]}{\sqrt{\operatorname{Var}\left[Y_{i j}^{o}\right]} \sqrt{\operatorname{Var}\left[Y_{i j^{\prime}}^{o}\right]}}
$$




\subsubsection{Estimação}

Para a inferência estatística no modelo DCRM-RE propomos usar o método da máxima verosimilhança. O vetor de parâmetros de interesse é $\boldsymbol{\theta}=\left(\boldsymbol{\beta}, \boldsymbol{\theta}_{\gamma}^{\top}, \boldsymbol{\theta}_{\epsilon}^{\top}\right)^{\top}$, em que $\boldsymbol{\theta}_{\gamma}$ de dimensão $p_{1}$, e $\boldsymbol{\theta}_{\epsilon}$ de dimensão $p_{2}$ são os correspondentes vetores de parâmetros associados às distribuições $f_{\gamma}$ e $f_{\epsilon}$, respectivamente. Assim, o espaço paramétrico é dado por

$$
\boldsymbol{\Theta}=\left(\boldsymbol{\theta} \in \mathbb{R}^{q} \mid \boldsymbol{\beta} \in \mathbb{R}^{p}, \boldsymbol{\theta}_{\gamma} \in \mathbb{R}^{p_{1}}, \boldsymbol{\theta}_{\epsilon} \in \mathbb{R}^{p_{2}}\right)^{\top}
$$

com $q=p+p_{1}+p_{2}$. A função de verossimilhança baseada na amostra observada $\mathbf{Y}^{o}=$ $\left(\mathbf{Y}_{1}^{o}, \ldots, \mathbf{Y}_{n}^{o}\right)^{\top}$ pode ser escrita como

$$
\begin{aligned}
L\left(\boldsymbol{\theta} ; \mathbf{Y}^{o}\right)=\prod_{i=1}^{n} & \int_{\mathbb{R}}\left\{\prod_{j=1}^{m_{i}}\left[\mathrm{P}\left(Y_{i j}^{o}=c_{0 i j}\right)\right]^{d_{0 i j}}\left[\mathrm{P}\left(Y_{i j}^{o}=c_{2 i j}\right)\right]^{d_{2 i j}}\right. \\
& \left.\times\left[f_{Y \mid \gamma}\left(y_{i j} \mid \gamma_{i} ; \boldsymbol{\beta}, \boldsymbol{\theta}_{\epsilon}\right)\right]^{1-d_{0 i j}-d_{2 i j}}\right\} f_{\gamma}\left(\gamma_{i} ; \boldsymbol{\theta}_{\gamma}\right) d \gamma_{i},
\end{aligned}
$$

Assim como no modelo linear misto normal-potência desenvolvido no Capítulo 3, a densidade marginal (5.3) e a função de verossimilhança (5.8) não têm expressões analíticas fechadas e a avaliação das integrais é feita usando o método de integração numérica de Gauss-Hermite unidimensional descrito na Seção 2.5.1.

\subsection{Modelo Normal-Potência Duplamente Censurado com Efei- tos Aleatórios}

Estudamos nesta seção o caso particular em que as variáveis aleatórias $\gamma_{i}$ e $\epsilon_{i j}$ no modelo (5.1) seguem uma distribuição normal-potência, isto é, $\gamma_{i} \stackrel{\mathrm{iid}}{\sim} \mathrm{PN}\left(0, \eta_{g}, \alpha_{g}\right)$ e $\epsilon_{i j} \stackrel{\mathrm{iid}}{\sim} \mathrm{PN}\left(0, \eta_{e}, \alpha_{e}\right)$, e portanto segue que $\boldsymbol{\theta}_{\gamma}=\left(\eta_{g}, \alpha_{g}\right)^{\top}$ e $\boldsymbol{\theta}_{\epsilon}=\left(\eta_{e}, \alpha_{e}\right)^{\top}$. Sob essa suposição, temos o modelo chamado de normal-potência duplamente censurado com efeitos aleatórios, e que nós denotaremos por modelo PNDC-RE (Power-Normal Doubly Censored with Random Effects).

As fdp's de $Y_{i j} \mid \gamma_{i}$ e $\gamma_{i}$ são dadas por

$$
\begin{aligned}
f_{Y \mid \gamma}\left(y_{i j} \mid \gamma_{i} ; \boldsymbol{\beta}, \boldsymbol{\theta}_{\epsilon}\right) & =\frac{\alpha_{e}}{\eta_{e}} \phi\left(\frac{y_{i j}-\mathbf{x}_{i j}^{\top} \boldsymbol{\beta}-\gamma_{i}}{\eta_{e}}\right)\left\{\Phi\left(\frac{y_{i j}-\mathbf{x}_{i j}^{\top} \boldsymbol{\beta}-\gamma_{i}}{\eta_{e}}\right)\right\}^{\alpha_{e}-1}, \quad \mathrm{e} \\
f_{\gamma}\left(\gamma_{i} ; \boldsymbol{\theta}_{\gamma}\right) & =\frac{\alpha_{g}}{\eta_{g}} \phi\left(\frac{\gamma_{i}}{\eta_{g}}\right)\left\{\Phi\left(\frac{\gamma_{i}}{\eta_{g}}\right)\right\}^{\alpha_{g}-1}
\end{aligned}
$$

respectivamente.

\subsubsection{Momentos no Modelo PNDC-RE}

No modelo PNDC-RE a média e a variância da observação $Y_{i j}^{o}$ e a covariância e a correlação entre as observações $Y_{i j}^{o}$ e $Y_{i j^{\prime}}^{o}$ são calculadas usando a Proposição 2. As probabilidades $p_{0 i j}, p_{1 i j}$ e $p_{2 i j}$ definidas acima são dadas por

$$
\begin{aligned}
& p_{0 i j}=\left\{\Phi\left(z_{0 i j}^{*}\right)\right\}^{\alpha_{e}}, \\
& p_{1 i j}=\left\{\Phi\left(z_{2 i j}^{*}\right)\right\}^{\alpha_{e}}-\left\{\Phi\left(z_{0 i j}^{*}\right)\right\}^{\alpha_{e}}, \\
& p_{2 i j}=1-\left\{\Phi\left(z_{2 i j}^{*}\right)\right\}^{\alpha_{e}},
\end{aligned}
$$


em que $\Phi(\cdot)$ é a função de distribuição acumulada (fda) da distribuição normal padrão, $z_{0 i j}^{*}=$ $\left(c_{0 i j}-\mathbf{x}_{i j}^{\top} \boldsymbol{\beta}-\gamma_{i}\right) / \eta_{e}$ e $z_{2 i j}^{*}=\left(c_{2 i j}-\mathbf{x}_{i j}^{\top} \boldsymbol{\beta}-\gamma_{i}\right) / \eta_{e}$. Então, de (5.4) e (5.5) temos que a média e a variância são dados por

$$
\mathrm{E}\left[Y_{i j}^{o}\right]=c_{0 i j} p_{0 i j}+c_{2 i j} p_{2 i j}+\eta_{e}\left(\frac{\mathbf{x}_{i j}^{\top} \boldsymbol{\beta}+\mu_{\gamma}}{\eta_{e}}+m_{i j}^{(1)}\right) p_{1 i j}
$$

e

$$
\begin{aligned}
\operatorname{Var}\left[Y_{i j}^{o}\right]=c_{0 i j}^{2} p_{0 i j} & \left(1-p_{0 i j}\right)+c_{2 i j}^{2} p_{2 i j}\left(1-p_{2 i j}\right)-2 c_{0 i j} c_{2 i j} p_{0 i j} p_{2 i j} \\
& +\eta_{e}^{2}\left\{\frac{\sigma_{\gamma}^{2}}{\eta_{e}^{2}}+\left(m_{i j}^{(2)}-\left[m_{i j}^{(1)}\right]^{2}\right)+\left(1-p_{1 i j}\right)\left(\frac{\mathbf{x}_{i j}^{\top} \boldsymbol{\beta}+\mu_{\gamma}}{\eta_{e}}+m_{i j}^{(1)}\right)^{2}\right. \\
& \left.-\frac{2}{\eta_{e}}\left(c_{0 i j} p_{0 i j}+c_{2 i j} p_{2 i j}\right)\left(\frac{\mathbf{x}_{i j}^{\top} \boldsymbol{\beta}+\mu_{\gamma}}{\eta_{e}}+m_{i j}^{(1)}\right)\right\} p_{1 i j},
\end{aligned}
$$

respectivamente; em que $\mu_{\gamma}=\eta_{g} m_{1}$, e $\sigma_{\gamma}^{2}=\eta_{g}^{2}\left(m_{2}-m_{1}^{2}\right)$ com

$$
m_{s}=\int_{0}^{1} \alpha_{g}\left[\Phi^{-1}(v)\right]^{r} v^{\alpha_{g}-1} d v, \quad s=1,2, \ldots
$$

e

$$
m_{i j}^{(r)}=\left(\left\{\Phi\left(z_{2 i j}^{*}\right)\right\}^{\alpha_{e}}-\left\{\Phi\left(z_{0 i j}^{*}\right)\right\}^{\alpha_{e}}\right)^{-1} \int_{\Phi\left(z_{0 i j}^{*}\right)}^{\Phi\left(z_{2 i j}^{*}\right)} \alpha_{e}\left[\Phi^{-1}(u)\right]^{r} u^{\alpha_{e}-1} d u, \quad r=1,2, \ldots
$$

A covariância e a correlação entre duas observações $Y_{i j}^{o}$ e $Y_{i j^{\prime}}^{o}$, nos momentos $j$ e $j^{\prime}\left(j \neq j^{\prime}\right)$ do $i$-ésimo indivíduo, são obtidas de (5.6) e (5.7) e ficam dadas por

$$
\operatorname{Cov}\left[Y_{i j}^{o}, Y_{i j^{\prime}}^{o}\right]=p_{1 i j} p_{1 i j^{\prime}} \sigma_{\gamma}^{2},
$$

$\mathrm{e}$

$$
\operatorname{Corr}\left[Y_{i j}^{o}, Y_{i j^{\prime}}^{o}\right]=\frac{p_{1 i j} p_{1 i j^{\prime}} \sigma_{\gamma}^{2}}{\sqrt{\operatorname{Var}\left[Y_{i j}^{o}\right]} \sqrt{\operatorname{Var}\left[Y_{i j^{\prime}}^{o}\right.}}
$$

respectivamente.

\subsubsection{Estimação no Modelo PNDC-RE}

Substituíndo as expressões para as fdp's $f_{Y}\left(y_{i j} \mid \gamma_{i} ; \boldsymbol{\beta}, \boldsymbol{\theta}_{\epsilon}\right)$ e $f_{\gamma}\left(\gamma_{i} ; \boldsymbol{\theta}_{\gamma}\right)$ dadas em (5.9) e (5.10) respectivamente, juntamente com as probabilidades $p_{0 i j}$ e $p_{2 i j}$ dadas em (5.11) e (5.13), a fdp marginal do vetor aleatório observado $\mathbf{Y}_{i}^{o}=\left(Y_{i 1}^{o}, \ldots, Y_{i m_{i}}^{o}\right)^{\top}$ é da forma

$$
\begin{aligned}
f\left(\mathbf{y}_{i}^{o} ; \boldsymbol{\theta}\right)=\int_{\mathbb{R}} & \left\{\prod_{j=1}^{m_{i}}\left[\Phi\left(\frac{c_{0 i j}-\mathbf{x}_{i j}^{\top} \boldsymbol{\beta}-\gamma_{i}}{\eta_{e}}\right)\right]^{d_{0 i j} \alpha_{e}}\left[1-\left\{\Phi\left(\frac{c_{2 i j}-\mathbf{x}_{i j}^{\top} \boldsymbol{\beta}-\gamma_{i}}{\eta_{e}}\right)\right\}^{\alpha_{e}}\right]^{d_{2 i j}}\right. \\
& \left.\times\left[\frac{\alpha_{e}}{\eta_{e}} \phi\left(\frac{y_{i j}-\mathbf{x}_{i j}^{\top} \boldsymbol{\beta}-\gamma_{i}}{\eta_{e}}\right)\left\{\Phi\left(\frac{y_{i j}-\mathbf{x}_{i j}^{\top} \boldsymbol{\beta}-\gamma_{i}}{\eta_{e}}\right)\right\}^{\alpha_{e}-1}\right]^{1-d_{0 i j}-d_{2 i j}}\right\} \\
& \times \frac{\alpha_{g}}{\eta_{g}} \phi\left(\frac{\gamma_{i}}{\eta_{g}}\right)\left\{\Phi\left(\frac{\gamma_{i}}{\eta_{g}}\right)\right\}^{\alpha_{g}-1} d \gamma_{i},
\end{aligned}
$$


em que $\boldsymbol{\theta}=\left(\boldsymbol{\beta}^{\top}, \boldsymbol{\theta}_{\epsilon}^{\top}, \boldsymbol{\theta}_{\gamma}^{\top}\right)^{\top}$ é o vetor de parâmetros de interesse, com $\boldsymbol{\theta}_{\epsilon}=\left(\eta_{e}, \alpha_{e}\right)^{\top}$ e $\boldsymbol{\theta}_{\gamma}=$ $\left(\eta_{g}, \alpha_{g}\right)^{\top}$. Procedendo como no modelo linear com interceptos aleatórios normal-potência, fazemos a mudança de variável $u_{i}=\gamma_{i} / \sqrt{2} \eta_{g}$ em (5.19), e obtemos a integral,

$$
\begin{aligned}
I_{i}=\int_{\mathbb{R}} & \left\{\prod_{j=1}^{m_{i}}\left[\Phi\left(\frac{c_{0 i j}-\mathbf{x}_{i j}^{\top} \boldsymbol{\beta}-\sqrt{2} \eta_{g} u_{i}}{\eta_{e}}\right)\right]^{d_{0 i j} \alpha_{e}}\left[1-\left\{\Phi\left(\frac{c_{2 i j}-\mathbf{x}_{i j}^{\top} \boldsymbol{\beta}-\sqrt{2} \eta_{g} u_{i}}{\eta_{e}}\right)\right\}^{\alpha_{e}}\right]^{d_{2 i j}}\right. \\
& \left.\times\left[\frac{\alpha_{e}}{\eta_{e}} \phi\left(\frac{y_{i j}-\mathbf{x}_{i j}^{\top} \boldsymbol{\beta}-\sqrt{2} \eta_{g} u_{i}}{\eta_{e}}\right)\left\{\Phi\left(\frac{y_{i j}-\mathbf{x}_{i j}^{\top} \boldsymbol{\beta}-\sqrt{2} \eta_{g} u_{i}}{\eta_{e}}\right)\right\}^{\alpha_{e}-1}\right]^{1-d_{0 i j}-d_{2 i j}}\right\} \\
& \times \frac{\alpha_{g}}{\sqrt{\pi}}\left\{\Phi\left(\sqrt{2} u_{i}\right)\right\}^{\alpha_{g}-1} \exp \left(-u_{i}^{2}\right) d u_{i},
\end{aligned}
$$

a qual tem a forma (2.23). Assim, usando a quadratura de Gauss-Hermite unidimensional temos que $I_{i}$ pode ser aproximada como

$$
\begin{aligned}
I_{i} \approx \sum_{k=1}^{Q} & \prod_{j=1}^{m_{i}}\left[\Phi\left(z_{0 i j}\right)\right]^{d_{0 i j} \alpha_{e}}\left[1-\left\{\Phi\left(z_{2 i j}\right)\right\}^{\alpha_{e}}\right]^{d_{2 i j}}\left[\frac{\alpha_{e}}{\eta_{e}} \phi\left(z_{i j}\right)\left\{\Phi\left(z_{i j}\right)\right\}^{\alpha_{e}-1}\right]^{1-d_{0 i j}-d_{2 i j}} \\
& \times\left\{\Phi\left(\sqrt{2} u_{k}\right)\right\}^{\alpha_{g}-1} \frac{\alpha_{g} \omega_{k}}{\sqrt{\pi}}
\end{aligned}
$$

em que $z_{0 i j}=\left(c_{0 i j}-\mathbf{x}_{i j}^{\top} \boldsymbol{\beta}-\sqrt{2} \eta_{g} u_{k}\right) / \eta_{e}, z_{2 i j}=\left(c_{2 i j}-\mathbf{x}_{i j}^{\top} \boldsymbol{\beta}-\sqrt{2} \eta_{g} u_{k}\right) / \eta_{e}$ e $z_{i j}=\left(y_{i j}-\mathbf{x}_{i j}^{\top} \boldsymbol{\beta}-\right.$ $\left.\sqrt{2} \eta_{g} u_{k}\right) / \eta_{e}$, com $u_{k}$ e $\omega_{k}$ sendo os pontos e os pesos da quadratura respectivamente.

A função de verossimilança aproximada para o vetor $\boldsymbol{\theta}=\left(\boldsymbol{\beta}^{\top}, \boldsymbol{\theta}_{\epsilon}^{\top}, \boldsymbol{\theta}_{\gamma}^{\top}\right)^{\top}$, baseada na amostra observada $\mathbf{Y}^{o}$, é obtida de (5.8) e toma a forma

$$
\begin{aligned}
L\left(\boldsymbol{\theta} ; \mathbf{Y}^{o}\right) \approx & \prod_{i=1}^{n}\left(\sum_{k=1}^{Q} \prod_{j=1}^{m_{i}}\left[\Phi\left(z_{0 i j}\right)\right]^{d_{0 i j} \alpha_{e}}\left[1-\left\{\Phi\left(z_{2 i j}\right)\right\}^{\alpha_{e}}\right]^{d_{2 i j}}\right. \\
& \left.\times\left[\frac{\alpha_{e}}{\eta_{e}} \phi\left(z_{i j}\right)\left\{\Phi\left(z_{i j}\right)\right\}^{\alpha_{e}-1}\right]^{1-d_{0 i j}-d_{2 i j}}\left\{\Phi\left(\sqrt{2} u_{k}\right)\right\}^{\alpha_{g}-1} \frac{\alpha_{g} \omega_{k}}{\sqrt{\pi}}\right),
\end{aligned}
$$

e o logaritmo da função de verossimilhança $\ell\left(\boldsymbol{\theta} ; \mathbf{Y}^{o}\right)$ fica dado por

$$
\begin{aligned}
\ell\left(\boldsymbol{\theta} ; \mathbf{Y}^{o}\right) \approx \sum_{i=1}^{n} & \log \left(\sum_{k=1}^{Q} \prod_{j=1}^{m_{i}}\left[\Phi\left(z_{0 i j}\right)\right]^{d_{0 i j} \alpha_{e}}\left[1-\left\{\Phi\left(z_{2 i j}\right)\right\}^{\alpha_{e}}\right]^{d_{2 i j}}\right. \\
& \left.\times\left[\frac{\alpha_{e}}{\eta_{e}} \phi\left(z_{i j}\right)\left\{\Phi\left(z_{i j}\right)\right\}^{\alpha_{e}-1}\right]^{1-d_{0 i j}-d_{2 i j}}\left\{\Phi\left(\sqrt{2} u_{k}\right)\right\}^{\alpha_{g}-1} \frac{\alpha_{g} \omega_{k}}{\sqrt{\pi}}\right) .
\end{aligned}
$$

A função escore aproximada, obtida diferenciando o logaritmo da função deverossimilhança $\ell(\boldsymbol{\theta} ; \mathbf{Y})$ em $(5.23)$, em relação a cada um dos parâmetros é dada por

$$
\boldsymbol{U}(\boldsymbol{\theta})=\left(\boldsymbol{U}_{\boldsymbol{\beta}}^{\top}(\boldsymbol{\theta}), U_{\alpha_{e}}(\boldsymbol{\theta}), U_{\eta_{e}}(\boldsymbol{\theta}), U_{\eta_{g}}(\boldsymbol{\theta}), U_{\alpha_{g}}(\boldsymbol{\theta})\right)^{\top}
$$

No Apêndice B.6.1 são apresentadas as expressões para os componentes do vetor $\boldsymbol{U}(\boldsymbol{\theta})$. O estimador de máxima verossimilhança de $\boldsymbol{\theta}=\left(\boldsymbol{\beta}^{\top}, \eta_{e}, \alpha_{e}, \eta_{g}, \alpha_{g}\right)^{\top}$ é obtido como solução ao sistema de equações $\boldsymbol{U}(\boldsymbol{\theta})=\mathbf{0}$. Note-se que os estimadores dos componentes de $\boldsymbol{\theta}$ não têm uma expressão fechada e precisam ser obtidos numericamente maximizando (5.23). 
Sob as condições usuais de regularidade, o estimador de máxima verossimilhança $\widehat{\boldsymbol{\theta}}$ é assintóticamente normal com média $\boldsymbol{\theta}$ e matriz de covariâncias $\boldsymbol{J}^{-1}(\boldsymbol{\theta})$, isto é,

$$
\sqrt{n}(\widehat{\boldsymbol{\theta}}-\boldsymbol{\theta}) \rightarrow N\left(\mathbf{0}, \boldsymbol{J}^{-1}(\boldsymbol{\theta})\right)
$$

em que $\boldsymbol{J}(\boldsymbol{\theta})$ é a matriz de informação observada, obtida como $\boldsymbol{J}(\boldsymbol{\theta})=-\partial^{2} \ell\left(\boldsymbol{\theta} ; \mathbf{Y}^{o}\right) / \partial \boldsymbol{\theta} \partial \boldsymbol{\theta}^{\top}$. Os elementos da matriz $\boldsymbol{J}(\boldsymbol{\theta})$ são apresentados no Apêndice B.6.2. Para obter os desvios padrão precisa-se obter também de forma numérica as estimativas dos elementos de $\boldsymbol{J}(\boldsymbol{\theta})$.

\subsubsection{Modelo Tobit Normal-Potência com Efeitos Aleatórios}

Martínez-Flórez et al. (2013b) estudaram uma extensão do modelo Tobit (Tobin, 1958) considerando que os erros seguem a distribuição normal-potência. Nesta seção estendemos o modelo proposto por Martínez-Flórez et al. (2013b) com a incorporação de um efeito aleatório $\gamma_{i}$ para o sujeito $i$, o qual segue uma distribuição normal-potência. o modelo resultante nós chamaremos de modelo tobit normal-potência com efeitos aleatórios, e é denotado por PNT-RE (Power-Normal Tobit with Random Effects).

O modelo PNT-RE é definido considerando a variável aleatória observada $Y_{i j}^{o}=D_{i j} Y_{i j}$, em que $D_{i j}=I\left(Y_{i j}>0\right)$ e $Y_{i j}=\mathbf{x}_{i j}^{\top} \boldsymbol{\beta}+\gamma_{i}+\epsilon_{i j}, \operatorname{com} \gamma_{i} \stackrel{\text { iid }}{\sim} \mathrm{PN}\left(0, \eta_{g}, \alpha_{g}\right)$ e $\epsilon_{i j} \stackrel{\text { iid }}{\sim} \mathrm{PN}\left(0, \eta_{e}, \alpha_{e}\right)$, para $i=1, \ldots, n$, e $j=1, \ldots, m_{i}$; isto é

$$
Y_{i j}^{o}= \begin{cases}\mathbf{x}_{i j}^{\top} \boldsymbol{\beta}+\gamma_{i}+\epsilon_{i j}, & \text { if } Y_{i j}>0 \\ 0, & \text { if } Y_{i j} \leq 0\end{cases}
$$

em que $Y_{i j}$ é o valor da $j$-ésima variável aleatória resposta para a $i$-ésima unidade amostral, para $i=1, \ldots, n$, e $j=1, \ldots, m_{i} ; \mathbf{x}_{i j}=\left(x_{i j 1}, \ldots, x_{i j p}\right)^{\top}$ é um vetor conhecido de dimensão $p \times 1$ de variáveis explicativas associadas aos efeitos fixos, para $i=1, \ldots, n$ e $j=1, \ldots, m_{i}$; $\boldsymbol{\beta}=\left(\beta_{1}, \ldots, \beta_{p}\right)^{\top}$ é um vetor de parâmetros desconhecido de dimensão $p \times 1 ; \gamma_{i}$ são variáveis aleatórias independentes igualmente distribuídas $\operatorname{PN}\left(0, \eta_{g}, \alpha_{g}\right)$ associadas à $i$-ésima unidade amostral; e os $\epsilon_{i j}$ 's são também variáveis aleatórias independentes igualmente distribuídas $\operatorname{PN}\left(0, \eta_{e}, \alpha_{e}\right)$ (independentes de $\left.\gamma_{i}\right)$.

A suposição acima é equivalente a considerar que dado $\gamma_{i}$, as variáveis aleatórias não observadas $Y_{i j}^{o}$ são independentes, $Y_{i j} \mid \gamma_{i} \stackrel{\text { ind }}{\sim} \mathrm{PN}\left(\mathbf{x}_{i j}^{\top} \boldsymbol{\beta}+\gamma_{i}, \eta_{e}, \alpha_{e}\right)$ com fdp dada por

$$
f_{Y \mid \gamma}\left(y_{i j} \mid \gamma_{i} ; \boldsymbol{\beta}, \eta_{e}, \alpha_{e}\right)=\frac{\alpha_{e}}{\eta_{e}} \phi\left(\frac{y_{i j}-\mathbf{x}_{i j}^{\top} \boldsymbol{\beta}-\gamma_{i}}{\eta_{e}}\right)\left\{\Phi\left(\frac{y_{i j}-\mathbf{x}_{i j}^{\top} \boldsymbol{\beta}-\gamma_{i}}{\eta_{e}}\right)\right\}^{\alpha_{e}-1}
$$

$i=1, \ldots, n$, em que

$$
f_{\gamma}\left(\gamma_{i} ; \eta_{g}, \alpha_{g}\right)=\frac{\alpha_{g}}{\eta_{g}} \phi\left(\frac{\gamma_{i}}{\eta_{g}}\right)\left\{\Phi\left(\frac{\gamma_{i}}{\eta_{g}}\right)\right\}^{\alpha_{g}-1}
$$

Para observacões $Y_{i j}^{o}$ iguais a zero, temos

$$
\mathrm{P}\left(Y_{i j}^{o}=0\right)=\left\{\Phi\left(-\frac{\mathbf{x}_{i}^{\top} \boldsymbol{\beta}+\gamma_{i}}{\eta_{e}}\right)\right\}^{\alpha_{e}}
$$

e para observações $Y_{i j}^{o}$ maiores que zero, tem-se que $Y_{i j}^{o} \sim \operatorname{PN}\left(\mathbf{x}_{i}^{\top} \boldsymbol{\beta}+\gamma_{i}, \eta_{e}, \alpha_{e}\right)$. Portanto a fdp 
marginal do vetor aleatório observado $\mathbf{Y}_{i}^{o}=\left(Y_{i 1}^{o}, \ldots, Y_{i m_{i}}^{o}\right)^{\top}$ é dado por

$$
\begin{aligned}
f\left(\mathbf{y}_{i}^{o} ; \boldsymbol{\theta}\right)=\int_{\mathbb{R}}\left\{\prod _ { j = 1 } ^ { m _ { i } } [ \Phi ( - \frac { \mathbf { x } _ { i } ^ { \top } \boldsymbol { \beta } + \gamma _ { i } } { \eta _ { e } } ) ] ^ { \alpha _ { e } ( 1 - d _ { i j } ) } \left[\frac{\alpha_{e}}{\eta_{e}} \phi\left(\frac{y_{i j}-\mathbf{x}_{i j}^{\top} \boldsymbol{\beta}-\gamma_{i}}{\eta_{e}}\right)\right.\right. \\
\left.\left.\times\left\{\Phi\left(\frac{y_{i j}-\mathbf{x}_{i j}^{\top} \boldsymbol{\beta}-\gamma_{i}}{\eta_{e}}\right)\right\}^{\alpha_{e}-1}\right]^{d_{i j}}\right\} \frac{\alpha_{g}}{\eta_{g}} \phi\left(\frac{\gamma_{i}}{\eta_{g}}\right)\left\{\Phi\left(\frac{\gamma_{i}}{\eta_{g}}\right)\right\}^{\alpha_{g}-1} d \gamma_{i},
\end{aligned}
$$

em que $\boldsymbol{\theta}=\left(\boldsymbol{\beta}^{\top}, \eta_{e}, \alpha_{e}, \eta_{g}, \alpha_{g}\right)^{\top}$, e

$$
d_{i j}=\left\{\begin{array}{lll}
1 & \text { se } & Y_{i j}^{o}>0 \\
0 & \text { se } & Y_{i j}^{o}=0
\end{array}\right.
$$

Segue que a função de verossimilhança baseada na amostra observada $\mathbf{Y}^{o}=\left(\mathbf{Y}_{1}^{o}, \ldots, \mathbf{Y}_{n}^{o}\right)^{\top}$ pode ser escrita como

$$
\begin{aligned}
L\left(\boldsymbol{\theta} ; \mathbf{Y}^{o}\right)= & \prod_{i=1}^{n} \int_{\mathbb{R}}\left\{\prod _ { j = 1 } ^ { m _ { i } } [ \Phi ( - \frac { \mathbf { x } _ { i } ^ { \top } \boldsymbol { \beta } + \gamma _ { i } } { \eta _ { e } } ) ] ^ { \alpha _ { e } ( 1 - d _ { i j } ) } \left[\frac{\alpha_{e}}{\eta_{e}} \phi\left(\frac{y_{i j}-\mathbf{x}_{i j}^{\top} \boldsymbol{\beta}-\gamma_{i}}{\eta_{e}}\right)\right.\right. \\
& \left.\left.\times\left\{\Phi\left(\frac{y_{i j}-\mathbf{x}_{i j}^{\top} \boldsymbol{\beta}-\gamma_{i}}{\eta_{e}}\right)\right\}^{\alpha_{e}-1}\right]^{d_{i j}}\right\} \frac{\alpha_{g}}{\eta_{g}} \phi\left(\frac{\gamma_{i}}{\eta_{g}}\right)\left\{\Phi\left(\frac{\gamma_{i}}{\eta_{g}}\right)\right\}^{\alpha_{g}-1} d \gamma_{i} .
\end{aligned}
$$

O modelo (5.26) é equivalente à situação mais geral em que o valor da censura associado à observação $i j$-ésima é substituído pelo valor $k_{i j}$ (um valor conhecido), isto é,

$$
Y_{i j}^{o}= \begin{cases}\mathbf{x}_{i j}^{\top} \boldsymbol{\beta}+\gamma_{i}+\epsilon_{i j}, & \text { if } Y_{i j}>k_{i j}, \\ k_{i j}, & \text { if } Y_{i j} \leq k_{i j}\end{cases}
$$

para $i=1, \ldots, n, j=1, \ldots, m_{i}$. Note-se que, fazendo $Y_{i j}^{o *}=Y_{i j}^{o}-k_{i j}, \mathbf{x}_{i j}^{*}=\left(\mathbf{x}_{i j}^{\top}, k_{i j}\right)^{\top} \mathrm{e}$ $\boldsymbol{\beta}^{*}=\left(\boldsymbol{\beta}^{\top},-1\right)^{\top}$, tem-se o modelo previo em (5.26). Assim, os resultados da inferência baseados no método da máxima verossimilhança podem ser usados para ajustar o modelo mais geral em (5.29).

\section{Momentos no Modelo PNT-RE}

Note-se que $Y_{i j}^{o}=D_{i j} Y_{i j}$, em que $Y_{i j}=\mu_{i j}+\gamma_{i}+\epsilon_{i j}$, com $\mu_{i j}=\mathrm{x}_{i j}^{\top} \boldsymbol{\beta}, \gamma_{i}=\eta_{g} W_{i}$ com $W_{i} \stackrel{\mathrm{iid}}{\sim} \mathrm{PN}\left(0,1, \alpha_{g}\right)$; e $\epsilon_{i j}=\eta_{e} Z_{i j} \operatorname{com} Z_{i j} \stackrel{\mathrm{iid}}{\sim} \mathrm{PN}\left(0,1, \alpha_{e}\right), i=1, \ldots, n, j=1, \ldots, m_{i}$.

Segue que a média e a variância da observação $Y_{i j}^{o}$ são dados por

$$
\begin{aligned}
\mathrm{E}\left[Y_{i j}^{o}\right] & =\mathrm{E}\left[Y_{i j} \mid Y_{i j}>0\right] \mathrm{P}\left(Y_{i j}>0\right) \\
& =\left(\mu_{i j}+\eta_{g} \mathrm{E}\left[W_{i}\right]+\eta_{e} \mathrm{E}\left[Z_{i j} \mid Z_{i j}+c_{i j}>0\right]\right)\left(1-\left\{\Phi\left(-c_{i j}\right)\right\}^{\alpha_{e}}\right)
\end{aligned}
$$


$\mathrm{e}$

$$
\begin{aligned}
\operatorname{Var}\left[Y_{i j}^{o}\right]= & \mathrm{E}\left[Y_{i j}^{2} \mid Y_{i j}>0\right] \mathrm{P}\left(Y_{i j}>0\right)-\left(\mathrm{E}\left[Y_{i j}^{o}\right]\right)^{2} \\
= & \eta_{e}^{2}\left\{\frac{\eta_{g}^{2} \operatorname{Var}\left[W_{i}\right]}{\eta_{e}^{2}}+\mathrm{E}\left[Z_{i j}^{2} \mid Z_{i j}+c_{i j}>0\right]-\left(\mathrm{E}\left[Z_{i j} \mid Z_{i j}+c_{i j}>0\right]\right)^{2}\right. \\
& \left.+\left\{\Phi\left(-c_{i j}\right)\right\}^{\alpha_{e}}\left(\frac{\mu_{i j}+\eta_{g} \mathrm{E}\left[W_{i}\right]}{\eta_{e}}+\eta_{e} \mathrm{E}\left[Z_{i j} \mid Z_{i j}+c_{i j}>0\right]\right)^{2}\right\}\left(1-\left\{\Phi\left(-c_{i j}\right)\right\}^{\alpha_{e}}\right),
\end{aligned}
$$

em que $c_{i j}=\left(\mu_{i j}+\gamma_{i}\right) / \eta_{e}$, e pela Proposição 2 , temos que

$$
\begin{aligned}
\mathrm{E}\left[Y_{i j}^{o}\right]= & \eta_{e}\left(\frac{\mu_{i j}+\mu_{\gamma}}{\eta_{e}}+m_{i j}^{(1)}\right)\left(1-\left\{\Phi\left(-c_{i j}\right)\right\}^{\alpha_{e}}\right) \\
\operatorname{Var}\left[Y_{i j}^{o}\right]=\eta_{e}^{2} & \left\{\frac{\sigma_{\gamma}^{2}}{\eta_{e}^{2}}+\left(m_{i j}^{(2)}-\left[m_{i j}^{(1)}\right]^{2}\right)\right. \\
& \left.+\left\{\Phi\left(-c_{i j}\right)\right\}^{\alpha_{e}}\left(\frac{\mu_{i j}+\mu_{\gamma}}{\eta_{e}}+m_{i j}^{(1)}\right)^{2}\right\}\left(1-\left\{\Phi\left(-c_{i j}\right)\right\}^{\alpha_{e}}\right)
\end{aligned}
$$

em que $\mu_{\gamma}=\eta_{g} m_{1}$ e $\sigma_{\gamma}=\eta_{g}^{2}\left(m_{2}-m_{1}^{2}\right)$, com $m_{1}$ e $m_{2}$ dados em (5.16);

$$
m_{i j}^{(r)}=\left(1-\left\{\Phi\left(-c_{i j}\right)\right\}^{\alpha_{e}}\right)^{-1} \int_{\Phi\left(-c_{i j}\right)}^{1} \alpha_{e}\left[\Phi^{-1}(u)\right]^{r} u^{\alpha_{e}-1} d u, \quad r=1,2, \ldots
$$

A covariância e a correlação entre duas observações $Y_{i j}^{o}$ e $Y_{i j^{\prime}}^{o}$, nos momentos $j$ e $j^{\prime}\left(j \neq j^{\prime}\right)$ do $i$-ésimo sujeito ficam dadas por

$$
\operatorname{Cov}\left[Y_{i j}^{o}, Y_{i j^{\prime}}^{o}\right]=\left(1-\left\{\Phi\left(-c_{i j}\right)\right\}^{\alpha_{e}}\right)\left(1-\left\{\Phi\left(-c_{i j^{\prime}}\right)\right\}^{\alpha_{e}}\right) \sigma_{\gamma}^{2}
$$

e

$$
\operatorname{Corr}\left[Y_{i j}^{o}, Y_{i j^{\prime}}^{o}\right]=\frac{\left(1-\left\{\Phi\left(-c_{i j}\right)\right\}^{\alpha_{e}}\right)\left(1-\left\{\Phi\left(-c_{i j^{\prime}}\right)\right\}^{\alpha_{e}}\right) \sigma_{\gamma}^{2}}{\sqrt{\operatorname{Var}\left[Y_{i j}^{o}\right]} \sqrt{\operatorname{Var}\left[Y_{i j^{\prime}}^{o}\right]}}
$$

respectivamente.

\section{Estimação no Modelo PNT-RE}

A estimação dos parâmetros no modelo PNT-RE é feita como no modelo PNDC-RE usando o método de máxima verossimilhança. O vetor de parâmetros de interesse é $\boldsymbol{\theta}=\left(\boldsymbol{\theta}, \eta_{e}, \alpha_{e}, \eta_{g}, \alpha_{g}\right)^{\top}$. Para a obtenção das estimativas desse vetor precisamos avaliar a integral

$$
\begin{aligned}
I_{i}=\int_{\mathbb{R}} & \left\{\prod _ { j = 1 } ^ { m _ { i } } [ \Phi ( - \frac { \mathbf { x } _ { i } ^ { \top } \boldsymbol { \beta } + \gamma _ { i } } { \eta _ { e } } ) ] ^ { \alpha _ { e } ( 1 - d _ { i j } ) } \left[\frac{\alpha_{e}}{\eta_{e}} \phi\left(\frac{y_{i j}-\mathbf{x}_{i j}^{\top} \boldsymbol{\beta}-\gamma_{i}}{\eta_{e}}\right)\right.\right. \\
& \left.\left.\times\left\{\Phi\left(\frac{y_{i j}-\mathbf{x}_{i j}^{\top} \boldsymbol{\beta}-\gamma_{i}}{\eta_{e}}\right)\right\}^{\alpha_{e}-1}\right]^{d_{i j}}\right\} \frac{\alpha_{g}}{\eta_{g}} \phi\left(\frac{\gamma_{i}}{\eta_{g}}\right)\left\{\Phi\left(\frac{\gamma_{i}}{\eta_{g}}\right)\right\}^{\alpha_{g}-1} d \gamma_{i},
\end{aligned}
$$


a qual, fazendo a mudança de variável $u_{i}=\gamma_{i} / \sqrt{2} \eta_{g}$ fica da forma

$$
\begin{aligned}
I_{i}=\int_{\mathbb{R}} & \left\{\prod _ { j = 1 } ^ { m _ { i } } [ \Phi ( - \frac { \mathbf { x } _ { i } ^ { \top } \boldsymbol { \beta } + \sqrt { 2 } \eta _ { g } u _ { i } } { \eta _ { e } } ) ] ^ { \alpha _ { e } ( 1 - d _ { i j } ) } \left[\frac{\alpha_{e}}{\eta_{e}} \phi\left(\frac{y_{i j}-\mathbf{x}_{i j}^{\top} \boldsymbol{\beta}-\sqrt{2} \eta_{g} u_{i}}{\eta_{e}}\right)\right.\right. \\
& \left.\left.\times\left\{\Phi\left(\frac{y_{i j}-\mathbf{x}_{i j}^{\top} \boldsymbol{\beta}-\sqrt{2} \eta_{g} u_{i}}{\eta_{e}}\right)\right\}^{\alpha_{e}-1}\right]^{d_{i j}}\right\}\left\{\Phi\left(\sqrt{2} u_{i}\right)\right\}^{\alpha_{g}-1} \frac{\alpha_{g}}{\sqrt{\pi}} \exp \left(-u_{i}^{2}\right) d u_{i}
\end{aligned}
$$

que tem a forma (2.23). Assim, usando a quadratura de Gauss-Hermite unidimensional temos que $I_{i}$ em (5.36) pode ser aproximada como

$$
\begin{gathered}
I_{i} \approx \sum_{k=1}^{Q} \prod_{j=1}^{m_{i}}\left[\Phi\left(-\frac{\mathbf{x}_{i}^{\top} \boldsymbol{\beta}+\sqrt{2} \eta_{g} u_{k}}{\eta_{e}}\right)\right]^{\alpha_{e}\left(1-d_{i j}\right)}\left[\frac{\alpha_{e}}{\eta_{e}} \phi\left(\frac{y_{i j}-\mathbf{x}_{i j}^{\top} \boldsymbol{\beta}-\sqrt{2} \eta_{g} u_{k}}{\eta_{e}}\right)\right. \\
\left.\times\left\{\Phi\left(\frac{y_{i j}-\mathbf{x}_{i j}^{\top} \boldsymbol{\beta}-\sqrt{2} \eta_{g} u_{k}}{\eta_{e}}\right)\right\}^{\alpha_{e}-1}\right]^{d_{i j}}\left\{\Phi\left(\sqrt{2} u_{k}\right)\right\}^{\alpha_{g}-1} \frac{\alpha_{g} \omega_{k}}{\sqrt{\pi}}
\end{gathered}
$$

Segue de (5.28) e (5.37) que o logaritmo da função de verossimilhança para o vetor $\boldsymbol{\theta}=$ $\left(\boldsymbol{\theta}, \eta_{e}, \alpha_{e}, \eta_{g}, \alpha_{g}\right)^{\top}$ baseada na amostra observada $\mathbf{Y}^{o}=\left(\mathbf{Y}_{1}^{o}, \ldots, \mathbf{Y}_{n}^{o}\right)^{\top}$ é da forma

$$
\begin{aligned}
\ell\left(\boldsymbol{\theta} ; \mathbf{Y}^{o}\right) \approx \sum_{i=1}^{n} & \log \left(\sum _ { k = 1 } ^ { Q } \prod _ { j = 1 } ^ { m _ { i } } [ \Phi ( - \frac { \mathbf { x } _ { i } ^ { \top } \boldsymbol { \beta } + \sqrt { 2 } \eta _ { g } u _ { k } } { \eta _ { e } } ) ] ^ { \alpha _ { e } ( 1 - d _ { i j } ) } \left[\frac{\alpha_{e}}{\eta_{e}} \phi\left(\frac{y_{i j}-\mathbf{x}_{i j}^{\top} \boldsymbol{\beta}-\sqrt{2} \eta_{g} u_{k}}{\eta_{e}}\right)\right.\right. \\
& \left.\left.\times\left\{\Phi\left(\frac{y_{i j}-\mathbf{x}_{i j}^{\top} \boldsymbol{\beta}-\sqrt{2} \eta_{g} u_{k}}{\eta_{e}}\right)\right\}^{\alpha_{e}-1}\right]^{d_{i j}}\left\{\Phi\left(\sqrt{2} u_{k}\right)\right\}^{\alpha_{g}-1} \frac{\alpha_{g} \omega_{k}}{\sqrt{\pi}}\right),
\end{aligned}
$$

Note-se que o modelo PNT-RE pode ser obtido também com um caso particular do modelo PNDC-RE quando fazemos $c_{0 i j}=0$ e $c_{2 i j} \longrightarrow+\infty$ para $i=1, \ldots, n$ e $j=1, \ldots, m_{i}$.

A função escore aproximada é obtida diferenciando o logaritmo da função deverossimilhança $\ell(\boldsymbol{\theta} ; \mathbf{Y})$ em $(5.38)$ em relação a cada um dos parâmetros e é dada por

$$
\boldsymbol{U}(\boldsymbol{\theta})=\left(\boldsymbol{U}_{\boldsymbol{\beta}}^{\top}(\boldsymbol{\theta}), U_{\eta_{e}}(\boldsymbol{\theta}), U_{\alpha_{e}}(\boldsymbol{\theta}), U_{\eta_{g}}(\boldsymbol{\theta}), U_{\alpha_{g}}(\boldsymbol{\theta})\right)^{\top}
$$

As expressões para os componentes do vetor $\boldsymbol{U}(\boldsymbol{\theta})$ podem ser obtidas a partir dos respectivos componentes da função escore do modelo PNDC-RE que foram dados no Apêndice B.6.1. O estimador de máxima verossimilhança de $\boldsymbol{\theta}=\left(\boldsymbol{\beta}^{\top}, \eta_{e}, \alpha_{e}, \eta_{g}, \alpha_{g}\right)^{\top}$ é obtido como solução ao sistema de equações $\boldsymbol{U}(\boldsymbol{\theta})=\mathbf{0}$ e devem ser obtidos numericamente maximizando (5.38).

Sob as condições usuais de regularidade, o estimador de máxima verossimilhança $\widehat{\theta}$ é assintóticamente normal com média $\boldsymbol{\theta}$ e matriz de covariâncias $\boldsymbol{J}^{-1}(\boldsymbol{\theta})$, isto é,

$$
\sqrt{n}(\widehat{\boldsymbol{\theta}}-\boldsymbol{\theta}) \rightarrow N\left(\mathbf{0}, \boldsymbol{J}^{-1}(\boldsymbol{\theta})\right)
$$

em que $\boldsymbol{J}(\boldsymbol{\theta})$ é a matriz de informação observada, obtida como $\boldsymbol{J}(\boldsymbol{\theta})=-\partial^{2} \ell\left(\boldsymbol{\theta} ; \mathbf{Y}^{o}\right) / \partial \boldsymbol{\theta} \partial \boldsymbol{\theta}^{\top}$.

\subsection{Modelo Normal-Assimétrico Duplamente Censurado com Efei- tos Aleatórios}

Uma alternativa ao modelo PNDC-RE resulta de considerar que as variáveis $\gamma_{i}$ e $\epsilon_{i j}$ no modelo (5.1) seguem uma distribuição assimétrica-normal, isto é, $\gamma_{i} \stackrel{\mathrm{iid}}{\sim} \mathrm{SN}\left(0, \eta_{g}, \lambda_{g}\right)$ e $\epsilon_{i j} \stackrel{\text { iid }}{\sim} \mathrm{SN}\left(0, \eta_{e}, \lambda_{e}\right)$, 
e nesse caso temos $\boldsymbol{\theta}_{\epsilon}=\left(\eta_{e}, \lambda_{e}\right)^{\top}$ e $\boldsymbol{\theta}_{\gamma}=\left(\eta_{g}, \lambda_{g}\right)^{\top}$. O modelo obtido é chamado de modelo normalassimétrico duplamente censurado com efeitos aleatórios, e é denotado por modelo SNDC-RE (Skew-Normal Doubly Censored with Random Effects). Se segue então que as fdp's de $Y_{i j} \mid \gamma_{i}$ e $\gamma_{i}$ são dadas por

$$
\begin{aligned}
f_{Y \mid \gamma}\left(y_{i j} \mid \gamma_{i} ; \boldsymbol{\beta}, \boldsymbol{\theta}_{\epsilon}\right) & =\frac{2}{\eta_{e}} \phi\left(\frac{y_{i j}-\mathbf{x}_{i j}^{\top} \boldsymbol{\beta}-\gamma_{i}}{\eta_{e}}\right) \Phi\left(\lambda_{e} \frac{y_{i j}-\mathbf{x}_{i j}^{\top} \boldsymbol{\beta}-\gamma_{i}}{\eta_{e}}\right), \quad \text { e } \\
f_{\gamma}\left(\gamma_{i} ; \boldsymbol{\theta}_{\gamma}\right) & =\frac{2}{\eta_{g}} \phi\left(\frac{\gamma_{i}}{\eta_{g}}\right) \Phi\left(\lambda_{g} \frac{\gamma_{i}}{\eta_{g}}\right)
\end{aligned}
$$

respectivamente.

\subsubsection{Momentos no Modelo SNDC-RE}

Definindo $z_{0 i j}^{*}=\left(c_{0 i j}-\mathbf{x}_{i j}^{\top} \boldsymbol{\beta}-\gamma_{i}\right) / \eta_{e}$ e $z_{2 i j}^{*}=\left(c_{2 i j}-\mathbf{x}_{i j}^{\top} \boldsymbol{\beta}-\gamma_{i}\right) / \eta_{e}$, as probabilidades $p_{0 i j}$, $p_{1 i j}$ e $p_{2 i j}$ ficam dadas por

$$
\begin{aligned}
& p_{0 i j}=F_{S N}\left(z_{0 i j}^{*}\right), \\
& p_{1 i j}=F_{S N}\left(z_{2 i j}^{*}\right)-F_{S N}\left(z_{0 i j}^{*}\right), \\
& p_{2 i j}=1-F_{S N}\left(z_{2 i j}^{*}\right),
\end{aligned}
$$

em que $F_{S N}(\cdot)$ é a fda da distribuição SN padrão com parâmetro de assimetría $\lambda_{e}, \operatorname{SN}\left(\lambda_{e}\right)$.

A média e a variância da observação $Y_{i j}^{o}$, bem como a covariância e a correlação entre as observações $Y_{i j}^{o}$ e $Y_{i j 1}^{o}$ no modelo SNDC-RE são calculadas procedendo como no modelo PNDCRE. De (5.4) e (5.5) junto com a Proposição 1 temos que a média e a variância de $Y_{i j}^{o}$ são dadas por

$$
\mathrm{E}\left[Y_{i j}^{o}\right]=c_{0 i j} p_{0 i j}+c_{2 i j} p_{2 i j}+\eta_{e}\left\{\frac{\mathbf{x}_{i j}^{\top} \boldsymbol{\beta}+\mu_{\gamma}}{\eta_{e}}+\mu_{i j}^{(1)}\right\} p_{1 i j}
$$

e

$$
\begin{aligned}
\operatorname{Var}\left[Y_{i j}^{o}\right]= & c_{0 i j}^{2} p_{0 i j}\left(1-p_{0 i j}\right)+c_{2 i j}^{2} p_{2 i j}\left(1-p_{2 i j}\right)-2 c_{0 i j} c_{2 i j} p_{0 i j} p_{2 i j} \\
& +\eta_{e}^{2}\left\{\frac{\sigma_{\gamma}^{2}}{\eta_{e}^{2}}+\left(\mu_{i j}^{(2)}-\left[\mu_{i j}^{(1)}\right]^{2}\right)+\left(1-p_{1 i j}\right)\left(\frac{\mathbf{x}_{i j}^{\top} \boldsymbol{\beta}+\mu_{\gamma}}{\eta_{e}}+\mu_{i j}^{(1)}\right)^{2}\right. \\
& \left.-\frac{2}{\eta_{e}}\left(c_{0 i j} p_{0 i j}+c_{2 i j} p_{2 i j}\right)\left(\frac{\mathbf{x}_{i j}^{\top} \boldsymbol{\beta}+\mu_{\gamma}}{\eta_{e}}+m_{i j}^{(1)}\right)\right\} p_{1 i j}
\end{aligned}
$$

com

$$
\begin{gathered}
\mu_{\gamma}=\eta_{g} \sqrt{\frac{2}{\pi}} \frac{\lambda_{g}}{\sqrt{1+\lambda_{g}^{2}}} \quad \text { e } \quad \sigma_{\gamma}^{2}=\eta_{g}^{2}\left(1-\frac{2}{\pi} \frac{\lambda_{g}^{2}}{1+\lambda_{g}^{2}}\right), \\
\mu_{i j}^{(1)}=\sqrt{\frac{2}{\pi}} \frac{\lambda_{e}}{\sqrt{1+\lambda_{e}^{2}}} \frac{\Phi\left(\lambda_{e}^{*} z_{2 i j}^{*}\right)-\Phi\left(\lambda_{e}^{*} z_{0 i j}^{*}\right)}{F_{S N}\left(z_{2 i j}^{*}\right)-F_{S N}\left(z_{0 i j}^{*}\right)}-\frac{f_{S N}\left(z_{2 i j}^{*}\right)-f_{S N}\left(z_{0 i j}^{*}\right)}{F_{S N}\left(z_{2 i j}^{*}\right)-F_{S N}\left(z_{0 i j}^{*}\right)}, \\
\mu_{i j}^{(2)}=1-\sqrt{\frac{2}{\pi}} \frac{\lambda_{e}}{1+\lambda_{e}^{2}} \frac{\phi\left(\lambda_{e}^{*} z_{2 i j}^{*}\right)-\phi\left(\lambda_{e}^{*} z_{0 i j}^{*}\right)}{F_{S N}\left(z_{2 i j}^{*}\right)-F_{S N}\left(z_{0 i j}^{*}\right)}-\frac{z_{2 i j}^{*} f_{S N}\left(z_{2 i j}^{*}\right)-z_{0 i j}^{*} f_{S N}\left(z_{0 i j}^{*}\right)}{F_{S N}\left(z_{2 i j}^{*}\right)-F_{S N}\left(z_{0 i j}^{*}\right)},
\end{gathered}
$$


em que $\lambda_{e}^{*}=\sqrt{1+\lambda_{e}^{2}}$.

A covariância e a correlação entre duas observações $Y_{i j}^{o}$ e $Y_{i j^{\prime}}^{o}$, nos momentos $j$ e $j^{\prime}\left(j \neq j^{\prime}\right)$ do $i$-ésimo indivíduo, são obtidas de (5.6) e (5.7) e ficam dadas por

$$
\operatorname{Cov}\left[Y_{i j}^{o}, Y_{i j^{\prime}}^{o}\right]=p_{1 i j} p_{1 i j^{\prime}} \sigma_{\gamma}^{2},
$$

e

$$
\operatorname{Corr}\left[Y_{i j}^{o}, Y_{i j^{\prime}}^{o}\right]=\frac{p_{1 i j} p_{1 i j^{\prime}} \sigma_{\gamma}^{2}}{\sqrt{\operatorname{Var}\left[Y_{i j}^{o}\right]} \sqrt{\operatorname{Var}\left[Y_{i j^{\prime}}^{o}\right]}},
$$

respectivamente, em que $\sigma_{\gamma}^{2}$ é dado em (5.46).

\subsubsection{Estimação no Modelo SNDC-RE}

A fdp marginal do vetor aleatório observado $\mathbf{Y}_{i}^{o}=\left(Y_{i 1}^{o}, \ldots, Y_{i m_{i}}^{o}\right)^{\top}$ é obtida substituíndo as expressões para as probabilidades $p_{0 i j}$ e $p_{2 i j}$ dadas em (5.43) e (5.45); e sob a suposição de $\gamma_{i} \stackrel{\mathrm{iid}}{\sim} \mathrm{SN}\left(0, \eta_{g}, \alpha_{g}\right)$ e $\epsilon_{i j} \stackrel{\text { iid }}{\sim} \mathrm{SN}\left(0, \eta_{e}, \alpha_{e}\right)$, temos que

$$
\begin{aligned}
f\left(\mathbf{y}_{i}^{o} ; \boldsymbol{\theta}\right)=\int_{\mathbb{R}} & \left\{\prod_{j=1}^{m_{i}}\left[F_{S N}\left(\frac{c_{0 i j}-\mathbf{x}_{i j}^{\top} \boldsymbol{\beta}-\gamma_{i}}{\eta_{e}}\right)\right]^{d_{0 i j}}\left[1-F_{S N}\left(\frac{c_{2 i j}-\mathbf{x}_{i j}^{\top} \boldsymbol{\beta}-\gamma_{i}}{\eta_{e}}\right)\right]^{d_{2 i j}}\right. \\
& \left.\times\left[\frac{2}{\eta_{e}} \phi\left(\frac{y_{i j}-\mathbf{x}_{i j}^{\top} \boldsymbol{\beta}-\gamma_{i}}{\eta_{e}}\right) \Phi\left(\lambda_{e} \frac{y_{i j}-\mathbf{x}_{i j}^{\top} \boldsymbol{\beta}-\gamma_{i}}{\eta_{e}}\right)\right]^{1-d_{0 i j}-d_{2 i j}}\right\} \\
& \times \frac{2}{\eta_{g}} \phi\left(\frac{\gamma_{i}}{\eta_{g}}\right) \Phi\left(\lambda_{g} \frac{\gamma_{i}}{\eta_{g}}\right) d \gamma_{i},
\end{aligned}
$$

em que $\boldsymbol{\theta}=\left(\boldsymbol{\beta}^{\top}, \boldsymbol{\theta}_{\epsilon}^{\top}, \boldsymbol{\theta}_{\gamma}^{\top}\right)^{\top}$ é o vetor de parâmetros de interesse, com $\boldsymbol{\theta}_{\epsilon}=\left(\eta_{e}, \lambda_{e}\right)^{\top}$ e $\boldsymbol{\theta}_{\gamma}=$ $\left(\eta_{g}, \lambda_{g}\right)^{\top}$. Fazendo a mudança de variável $u_{i}=\gamma_{i} / \sqrt{2} \eta_{g}$ em (5.49) obtemos a integral,

$$
\begin{aligned}
I_{i}=\int_{\mathbb{R}} & \left\{\prod_{j=1}^{m_{i}}\left[F_{S N}\left(\frac{c_{0 i j}-\mathbf{x}_{i j}^{\top} \boldsymbol{\beta}-\sqrt{2} \eta_{g} u_{i}}{\eta_{e}}\right)\right]^{d_{0 i j}}\left[1-F_{S N}\left(\frac{c_{2 i j}-\mathbf{x}_{i j}^{\top} \boldsymbol{\beta}-\sqrt{2} \eta_{g} u_{i}}{\eta_{e}}\right)\right]^{d_{2 i j}}\right. \\
& \left.\times\left[\frac{2}{\eta_{e}} \phi\left(\frac{y_{i j}-\mathbf{x}_{i j}^{\top} \boldsymbol{\beta}-\sqrt{2} \eta_{g} u_{i}}{\eta_{e}}\right) \Phi\left(\lambda_{e} \frac{y_{i j}-\mathbf{x}_{i j}^{\top} \boldsymbol{\beta}-\sqrt{2} \eta_{g} u_{i}}{\eta_{e}}\right)\right]^{1-d_{0 i j}-d_{2 i j}}\right\} \\
& \times \frac{2}{\sqrt{\pi}} \Phi\left(\sqrt{2} \lambda_{g} u_{i}\right) \exp \left(-u_{i}^{2}\right) d u_{i},
\end{aligned}
$$

a qual tem a forma (2.23). Assim usando a quadratura de Gauss-Hermite unidimensional temos que $I_{i}$ pode ser aproximada como

$$
\begin{aligned}
I_{i} \approx \sum_{k=1}^{Q} & \prod_{j=1}^{m_{i}}\left[F_{S N}\left(z_{0 i j}\right)\right]^{d_{0 i j}}\left[1-F_{S N}\left(z_{2 i j}\right)\right]^{d_{2 i j}}\left[\frac{2}{\eta_{e}} \phi\left(z_{i j}\right) \Phi\left(\lambda_{e} z_{i j}\right)\right]^{1-d_{0 i j}-d_{2 i j}} \\
& \times \Phi\left(\sqrt{2} \lambda_{g} u_{k}\right) \frac{2 \omega_{k}}{\sqrt{\pi}}
\end{aligned}
$$

em que $z_{0 i j}=\left(c_{0 i j}-\mathbf{x}_{i j}^{\top} \boldsymbol{\beta}-\sqrt{2} \eta_{g} u_{k}\right) / \eta_{e}, z_{2 i j}=\left(c_{2 i j}-\mathbf{x}_{i j}^{\top} \boldsymbol{\beta}-\sqrt{2} \eta_{g} u_{k}\right) / \eta_{e}$ e $z_{i j}=\left(y_{i j}-\mathbf{x}_{i j}^{\top} \boldsymbol{\beta}-\right.$ $\left.\sqrt{2} \eta_{g} u_{k}\right) / \eta_{e}$, com $u_{k}$ e $\omega_{k}$ sendo os pontos e os pesos da quadratura respectivamente.

A função de verossimilhnaça aproximada para o vetor $\boldsymbol{\theta}=\left(\boldsymbol{\beta}^{\top}, \boldsymbol{\theta}_{\epsilon}^{\top}, \boldsymbol{\theta}_{\gamma}^{\top}\right)^{\top}$, baseados na amos- 
tra observada $\mathbf{Y}^{o}$, é obtida de (5.8) e toma a forma

$$
\begin{aligned}
L\left(\boldsymbol{\theta} ; \mathbf{Y}^{o}\right) \approx & \prod_{i=1}^{n}\left(\sum_{k=1}^{Q} \prod_{j=1}^{m_{i}}\left[F_{S N}\left(z_{0 i j}\right)\right]^{d_{0 i j}}\left[1-\left\{F_{S N}\left(z_{2 i j}\right)\right\}\right]^{d_{2 i j}}\left[\frac{2}{\eta_{e}} \phi\left(z_{i j}\right) \Phi\left(\lambda_{e} z_{i j}\right)\right]^{1-d_{0 i j}-d_{2 i j}}\right. \\
& \left.\times \Phi\left(\sqrt{2} \lambda_{g} u_{k}\right) \frac{2 \omega_{k}}{\sqrt{\pi}}\right)
\end{aligned}
$$

e o logaritmo da função de verossimilhança $\ell\left(\boldsymbol{\theta} ; \mathbf{Y}^{o}\right)$ fica dado por

$$
\begin{aligned}
\ell\left(\boldsymbol{\theta} ; \mathbf{Y}^{o}\right) \approx \sum_{i=1}^{n} & \log \left(\sum_{k=1}^{Q} \prod_{j=1}^{m_{i}}\left[F_{S N}\left(z_{0 i j}\right)\right]^{d_{0 i j}}\left[1-\left\{F_{S N}\left(z_{2 i j}\right)\right\}\right]^{d_{2 i j}}\left[\frac{2}{\eta_{e}} \phi\left(z_{i j}\right) \Phi\left(\lambda_{e} z_{i j}\right)\right]^{1-d_{0 i j}-d_{2 i j}}\right. \\
& \left.\times \Phi\left(\sqrt{2} \lambda_{g} u_{k}\right) \frac{2 \omega_{k}}{\sqrt{\pi}}\right)
\end{aligned}
$$

A função escore aproximada é obtida diferenciando o logaritmo da função de verossimilhança $\ell(\boldsymbol{\theta} ; \mathbf{Y})$ em (5.53) em relação a cada um dos parâmetros e é dada por

$$
\boldsymbol{U}(\boldsymbol{\theta})=\left(\boldsymbol{U}_{\boldsymbol{\beta}}^{\top}(\boldsymbol{\theta}), U_{\eta_{e}}(\boldsymbol{\theta}), U_{\lambda_{e}}(\boldsymbol{\theta}), U_{\eta_{g}}(\boldsymbol{\theta}), U_{\lambda_{g}}(\boldsymbol{\theta})\right)^{\top}
$$

O estimador de máxima verossimilhança de $\boldsymbol{\theta}=\left(\boldsymbol{\beta}^{\top}, \eta_{e}, \lambda_{e}, \eta_{g}, \lambda_{g}\right)^{\top}$ é obtido como solução ao sistema de equações $\boldsymbol{U}(\boldsymbol{\theta})=\mathbf{0}$. Note-se que os estimadores dos componentes de $\boldsymbol{\theta}$ não têm uma expressão fechada e precisam ser obtidos numericamente maximizando (5.53).

\subsubsection{Modelo Tobit Normal-Assimétrico com Efeitos Aleatórios}

Quando no modelo (5.26) substituímos a suposição de distribuição normal-potência para as variáveis aleatórias $\gamma_{i}$ e $\epsilon_{i j}$ pela suposição de distribuição normal-assimétrica, o modelo resultante é chamado de modelo tobit normal-assimétrico com efeitos aleatórios, e é denotado por SNTRE (Skew-normal Tobit with Random Effects). Assim, procedendo como no modelo PNT-RE, consideramos a variável aleatória observada $Y_{i j}^{o}=D_{i j} Y_{i j}$, em que $D_{i j}=I\left(Y_{i j}>0\right)$ e $Y_{i j}=$ $\mathbf{x}_{i j}^{\top} \boldsymbol{\beta}+\gamma_{i}+\epsilon_{i j}, \operatorname{com} \gamma_{i} \stackrel{\text { iid }}{\sim} \mathrm{SN}\left(0, \eta_{g}, \lambda_{g}\right)$ e $\epsilon_{i j} \stackrel{\text { iid }}{\sim} \operatorname{SN}\left(0, \eta_{e}, \lambda_{e}\right)$, para $i=1, \ldots, n$, e $j=1, \ldots, m_{i}$.

Se segue então que $Y_{i j} \mid \gamma_{i} \stackrel{\text { ind }}{\sim} \mathrm{SN}\left(\mathbf{x}_{i j}^{\top} \boldsymbol{\beta}+\gamma_{i}, \eta_{e}, \lambda_{e}\right)$, com fdp dada por

$$
f_{Y \mid \gamma}\left(y_{i j} \mid \gamma_{i} ; \boldsymbol{\beta}, \eta_{e}, \lambda_{e}\right)=\frac{2}{\eta_{e}} \phi\left(\frac{y_{i j}-\mathbf{x}_{i j}^{\top} \boldsymbol{\beta}-\gamma_{i}}{\eta_{e}}\right) \Phi\left(\lambda_{e} \frac{y_{i j}-\mathbf{x}_{i j}^{\top} \boldsymbol{\beta}-\gamma_{i}}{\eta_{e}}\right)
$$

$i=1, \ldots, n, \mathrm{e}$

$$
f_{\gamma}\left(\gamma_{i} ; \eta_{g}, \alpha_{g}\right)=\frac{2}{\eta_{g}} \phi\left(\frac{\gamma_{i}}{\eta_{g}}\right) \Phi\left(\lambda_{g} \frac{\gamma_{i}}{\eta_{g}}\right)
$$

Para observacões $Y_{i j}^{o}$ iguais a zero, temos

$$
\mathrm{P}\left(Y_{i j}^{o}=0\right)=F_{S N}\left(-\frac{\mathbf{x}_{i}^{\top} \boldsymbol{\beta}+\gamma_{i}}{\eta_{e}}\right),
$$

e para observações $Y_{i j}^{o}$ maiores que zero, tem-se que $Y_{i j}^{o} \stackrel{\text { ind }}{\sim} \operatorname{SN}\left(\mathbf{x}_{i j}^{\top} \boldsymbol{\beta}+\gamma_{i}, \eta_{e}, \lambda_{e}\right)$. Portanto a fdp 
marginal do vetor aleatório observado $\mathbf{Y}_{i}^{o}=\left(Y_{i 1}^{o}, \ldots, Y_{i m_{i}}^{o}\right)^{\top}$ é dado por

$$
\begin{aligned}
f\left(\mathbf{y}_{i}^{o} ; \boldsymbol{\theta}\right)=\int_{\mathbb{R}} & \left\{\prod _ { j = 1 } ^ { m _ { i } } [ F _ { S N } ( - \frac { \mathbf { x } _ { i } ^ { \top } \boldsymbol { \beta } + \gamma _ { i } } { \eta _ { e } } ) ] ^ { ( 1 - d _ { i j } ) } \left[\frac{2}{\eta_{e}} \phi\left(\frac{y_{i j}-\mathbf{x}_{i j}^{\top} \boldsymbol{\beta}-\gamma_{i}}{\eta_{e}}\right)\right.\right. \\
& \left.\left.\times \Phi\left(\lambda_{e} \frac{y_{i j}-\mathbf{x}_{i j}^{\top} \boldsymbol{\beta}-\gamma_{i}}{\eta_{e}}\right)\right]^{d_{i j}}\right\} \frac{2}{\eta_{g}} \phi\left(\frac{\gamma_{i}}{\eta_{g}}\right) \Phi\left(\lambda_{g} \frac{\gamma_{i}}{\eta_{g}}\right) d \gamma_{i},
\end{aligned}
$$

em que $\boldsymbol{\theta}=\left(\boldsymbol{\beta}^{\top}, \eta_{e}, \lambda_{e}, \eta_{g}, \lambda_{g}\right)^{\top}$, e

$$
d_{i j}=\left\{\begin{array}{lll}
1 & \text { se } & Y_{i j}^{o}>0 \\
0 & \text { se } & Y_{i j}^{o}=0
\end{array}\right.
$$

Segue que a função de verossimilhança baseada na amostra observada $\mathbf{Y}^{o}=\left(\mathbf{Y}_{1}^{o}, \ldots, \mathbf{Y}_{n}^{o}\right)^{\top}$ pode ser escrita como

$$
\begin{aligned}
L\left(\boldsymbol{\theta} ; \mathbf{Y}^{o}\right)=\prod_{i=1}^{n} & \int_{\mathbb{R}}\left\{\prod _ { j = 1 } ^ { m _ { i } } [ F _ { S N } ( - \frac { \mathbf { x } _ { i } ^ { \top } \boldsymbol { \beta } + \gamma _ { i } } { \eta _ { e } } ) ] ^ { ( 1 - d _ { i j } ) } \left[\frac{2}{\eta_{e}} \phi\left(\frac{y_{i j}-\mathbf{x}_{i j}^{\top} \boldsymbol{\beta}-\gamma_{i}}{\eta_{e}}\right)\right.\right. \\
& \left.\left.\times \Phi\left(\lambda_{e} \frac{y_{i j}-\mathbf{x}_{i j}^{\top} \boldsymbol{\beta}-\gamma_{i}}{\eta_{e}}\right)\right]^{d_{i j}}\right\} \frac{2}{\eta_{g}} \phi\left(\frac{\gamma_{i}}{\eta_{g}}\right) \Phi\left(\lambda_{g} \frac{\gamma_{i}}{\eta_{g}}\right) d \gamma_{i},
\end{aligned}
$$

\section{Momentos no Modelo SNT-RE}

A média e a variância do valor observado $Y_{i j}^{o}$ no modelo SNT-RE são calculados desde que $Y_{i j}^{o}=D_{i j} Y_{i j}$, em que $Y_{i j}=\mu_{i j}+\gamma_{i}+\epsilon_{i j}$, com $\mu_{i j}=\mathrm{x}_{i j}^{\top} \boldsymbol{\beta}, \gamma_{i}=\eta_{g} W_{i} \operatorname{com} W_{i} \stackrel{\mathrm{iid}}{\sim} \operatorname{SN}\left(0,1, \lambda_{g}\right)$; e $\epsilon_{i j}=\eta_{e} Z_{i j} \operatorname{com} Z_{i j} \stackrel{\mathrm{iid}}{\sim} \mathrm{SN}\left(0,1, \lambda_{e}\right), i=1, \ldots, n, j=1, \ldots, m_{i}$, assim

$$
\begin{aligned}
\mathrm{E}\left[Y_{i j}^{o}\right] & =\mathrm{E}\left[Y_{i j} \mid Y_{i j}>0\right] \mathrm{P}\left(Y_{i j}>0\right) \\
& =\left(\mu_{i j}+\eta_{g} \mathrm{E}\left[W_{i}\right]+\eta_{e} \mathrm{E}\left[Z_{i j} \mid Z_{i j}+c_{i j}>0\right]\right)\left(1-F_{S N}\left(-c_{i j}\right)\right)
\end{aligned}
$$

e

$$
\begin{aligned}
\operatorname{Var}\left[Y_{i j}^{o}\right]= & \mathrm{E}\left[Y_{i j}^{2} \mid Y_{i j}>0\right] \mathrm{P}\left(Y_{i j}>0\right)-\left(\mathrm{E}\left[Y_{i j}^{o}\right]\right)^{2} \\
= & \eta_{e}^{2}\left\{\frac{\eta_{g}^{2} \operatorname{Var}\left[W_{i}\right]}{\eta_{e}^{2}}+\mathrm{E}\left[Z_{i j}^{2} \mid Z_{i j}+c_{i j}>0\right]-\left(\mathrm{E}\left[Z_{i j} \mid Z_{i j}+c_{i j}>0\right]\right)^{2}\right. \\
& \left.\quad+F_{S N}\left(-c_{i j}\right)\left(\frac{\mu_{i j}+\eta_{g} \mathrm{E}\left[W_{i}\right]}{\eta_{e}}+\eta_{e} \mathrm{E}\left[Z_{i j} \mid Z_{i j}+c_{i j}>0\right]\right)^{2}\right\}\left(1-F_{S N}\left(-c_{i j}\right)\right),
\end{aligned}
$$

em que $c_{i j}=\left(\mu_{i j}+\gamma_{i}\right) / \eta_{e}$, e pela Proposição 1 , temos que

$$
\begin{aligned}
\mathrm{E}\left[Y_{i j}^{o}\right]= & \eta_{e}\left(\frac{\mu_{i j}+\mu_{\gamma}}{\eta_{e}}+\mu_{i j}^{(1)}\right)\left(1-F_{S N}\left(-c_{i j}\right)\right) \\
\operatorname{Var}\left[Y_{i j}^{o}\right]=\eta_{e}^{2} & \left\{\frac{\sigma_{\gamma}^{2}}{\eta_{e}^{2}}+\left(\mu_{i j}^{(2)}-\left[\mu_{i j}^{(1)}\right]^{2}\right)\right. \\
& \left.+F_{S N}\left(-c_{i j}\right)\left(\frac{\mu_{i j}+\mu_{\gamma}}{\eta_{e}}+\mu_{i j}^{(1)}\right)^{2}\right\}\left(1-F_{S N}\left(-c_{i j}\right)\right)
\end{aligned}
$$


em que $\mu_{\gamma}$ e $\sigma_{\gamma}^{2}$ são dados em (5.46); e

$$
\begin{aligned}
\mu_{i j}^{(1)} & =\sqrt{\frac{2}{\pi}} \frac{\lambda_{e}}{\sqrt{1+\lambda_{e}^{2}}} \frac{\Phi\left(\lambda_{e}^{*} c_{i j}\right)}{1-F_{S N}\left(-c_{i j}\right)}+\frac{f_{S N}\left(c_{i j}\right)}{1-F_{S N}\left(-c_{i j}\right)} \\
\mu_{i j}^{(2)} & =1-\sqrt{\frac{2}{\pi}} \frac{\lambda_{e}}{1+\lambda_{e}^{2}} \frac{\phi\left(\lambda_{e}^{*} c_{i j}\right)}{1-F_{S N}\left(-c_{i j}\right)}-\frac{c_{i j} f_{S N}\left(c_{i j}\right)}{1-F_{S N}\left(-c_{i j}\right)}
\end{aligned}
$$

em que $\lambda_{e}^{*}=\sqrt{1+\lambda_{e}^{2}}$.

A covariância e a correlação entre duas observações $Y_{i j}^{o}$ e $Y_{i j^{\prime}}^{o}$, nos momentos $j$ e $j^{\prime}\left(j \neq j^{\prime}\right)$ do $i$-ésimo sujeito ficam dadas por

$$
\operatorname{Cov}\left[Y_{i j}^{o}, Y_{i j^{\prime}}^{o}\right]=\left(1-F_{S N}\left(-c_{i j}\right)\right)\left(1-F_{S N}\left(-c_{i j^{\prime}}\right)\right) \sigma_{\gamma}^{2}
$$

e

$$
\operatorname{Corr}\left[Y_{i j}^{o}, Y_{i j^{\prime}}^{o}\right]=\frac{\left(1-F_{S N}\left(-c_{i j}\right)\right)\left(1-F_{S N}\left(-c_{i j^{\prime}}\right)\right) \sigma_{\gamma}^{2}}{\sqrt{\operatorname{Var}\left[Y_{i j}^{o}\right]} \sqrt{\operatorname{Var}\left[Y_{i j^{\prime}}^{o}\right]}},
$$

respectivamente.

\section{Estimação no Modelo SNT-RE}

Para a inferência estatística no modelo SNT-RE propomos o método da máxima verossimilhança. O vetor de parâmetros de interesse é $\boldsymbol{\theta}=\left(\boldsymbol{\theta}, \eta_{e}, \lambda_{e}, \eta_{g}, \lambda_{g}\right)^{\top}$, e para a obtenção das estimativas do vetor $\boldsymbol{\theta}$ precisamos avaliar a integral

$$
\begin{aligned}
I_{i}=\int_{\mathbb{R}} & \left\{\prod _ { j = 1 } ^ { m _ { i } } [ F _ { S N } ( - \frac { \mathbf { x } _ { i } ^ { \top } \boldsymbol { \beta } + \gamma _ { i } } { \eta _ { e } } ) ] ^ { ( 1 - d _ { i j } ) } \left[\frac{2}{\eta_{e}} \phi\left(\frac{y_{i j}-\mathbf{x}_{i j}^{\top} \boldsymbol{\beta}-\gamma_{i}}{\eta_{e}}\right)\right.\right. \\
& \left.\left.\times \Phi\left(\lambda_{e} \frac{y_{i j}-\mathbf{x}_{i j}^{\top} \boldsymbol{\beta}-\gamma_{i}}{\eta_{e}}\right)\right]^{d_{i j}}\right\} \frac{2}{\eta_{g}} \phi\left(\frac{\gamma_{i}}{\eta_{g}}\right) \Phi\left(\lambda_{g} \frac{\gamma_{i}}{\eta_{g}}\right) d \gamma_{i},
\end{aligned}
$$

a qual fazendo a mudança de variável $u_{i}=\gamma_{i} / \sqrt{2} \eta_{g}$ fica da forma

$$
\begin{aligned}
I_{i}=\int_{\mathbb{R}} & \left\{\prod _ { j = 1 } ^ { m _ { i } } [ F _ { S N } ( - \frac { \mathbf { x } _ { i } ^ { \top } \boldsymbol { \beta } + \sqrt { 2 } \eta _ { g } u _ { i } } { \eta _ { e } } ) ] ^ { ( 1 - d _ { i j } ) } \left[\frac{2}{\eta_{e}} \phi\left(\frac{y_{i j}-\mathbf{x}_{i j}^{\top} \boldsymbol{\beta}-\sqrt{2} \eta_{g} u_{i}}{\eta_{e}}\right)\right.\right. \\
& \left.\left.\times \Phi\left(\lambda_{e} \frac{y_{i j}-\mathbf{x}_{i j}^{\top} \boldsymbol{\beta}-\sqrt{2} \eta_{g} u_{i}}{\eta_{e}}\right)\right]^{d_{i j}}\right\} \Phi\left(\sqrt{2} \lambda_{g} u_{i}\right) \frac{2}{\sqrt{\pi}} \exp \left(-u_{i}^{2}\right) d u_{i}
\end{aligned}
$$

Usando a quadratura de Gauss-Hermite unidimensional temos que $I_{i}$ em (5.63) pode ser aproximada como

$$
\begin{aligned}
I_{i} \approx \sum_{k=1}^{Q} & \prod_{j=1}^{m_{i}}\left[F_{S N}\left(-\frac{\mathbf{x}_{i}^{\top} \boldsymbol{\beta}+\sqrt{2} \eta_{g} u_{k}}{\eta_{e}}\right)\right]^{\left(1-d_{i j}\right)}\left[\frac{2}{\eta_{e}} \phi\left(\frac{y_{i j}-\mathbf{x}_{i j}^{\top} \boldsymbol{\beta}-\sqrt{2} \eta_{g} u_{k}}{\eta_{e}}\right)\right. \\
& \left.\times \Phi\left(\lambda_{e} \frac{y_{i j}-\mathbf{x}_{i j}^{\top} \boldsymbol{\beta}-\sqrt{2} \eta_{g} u_{k}}{\eta_{e}}\right)\right]^{d_{i j}} \Phi\left(\sqrt{2} \lambda_{g} u_{k}\right) \frac{2 \omega_{k}}{\sqrt{\pi}}
\end{aligned}
$$


Segue de (5.56) e (5.64) que o logaritmo da função de verossimilhança para o vetor $\boldsymbol{\theta}=$ $\left(\boldsymbol{\theta}, \eta_{e}, \lambda_{e}, \eta_{g}, \lambda_{g}\right)^{\top}$ baseada na amostra observada $\mathbf{Y}^{o}=\left(\mathbf{Y}_{1}^{o}, \ldots, \mathbf{Y}_{n}^{o}\right)^{\top}$ é da forma

$$
\begin{aligned}
\ell\left(\boldsymbol{\theta} ; \mathbf{Y}^{o}\right) \approx \sum_{i=1}^{n} & \log \left(\sum _ { k = 1 } ^ { Q } \prod _ { j = 1 } ^ { m _ { i } } [ F _ { S N } ( - \frac { \mathbf { x } _ { i } ^ { \top } \boldsymbol { \beta } + \sqrt { 2 } \eta _ { g } u _ { k } } { \eta _ { e } } ) ] ^ { ( 1 - d _ { i j } ) } \left[\frac{2}{\eta_{e}} \phi\left(\frac{y_{i j}-\mathbf{x}_{i j}^{\top} \boldsymbol{\beta}-\sqrt{2} \eta_{g} u_{k}}{\eta_{e}}\right)\right.\right. \\
& \left.\left.\times \Phi\left(\lambda_{e} \frac{y_{i j}-\mathbf{x}_{i j}^{\top} \boldsymbol{\beta}-\sqrt{2} \eta_{g} u_{k}}{\eta_{e}}\right)\right]^{d_{i j}} \Phi\left(\sqrt{2} \lambda_{g} u_{k}\right) \frac{2 \omega_{k}}{\sqrt{\pi}}\right)
\end{aligned}
$$

A função escore aproximada é obtida diferenciando o logaritmo da função deverossimilhança $\ell(\boldsymbol{\theta} ; \mathbf{Y})$ em $(5.65)$, em relação a cada um dos parâmetros e é dada por

$$
\boldsymbol{U}(\boldsymbol{\theta})=\left(\boldsymbol{U}_{\boldsymbol{\beta}}^{\top}(\boldsymbol{\theta}), U_{\eta_{e}}(\boldsymbol{\theta}), U_{\lambda_{e}}(\boldsymbol{\theta}), U_{\eta_{g}}(\boldsymbol{\theta}), U_{\lambda_{g}}(\boldsymbol{\theta})\right)^{\top} .
$$

O estimador de máxima verossimilhança de $\boldsymbol{\theta}=\left(\boldsymbol{\beta}^{\top}, \eta_{e}, \lambda_{e}, \eta_{g}, \lambda_{g}\right)^{\top}$ é obtido como solução ao sistema de equações $\boldsymbol{U}(\boldsymbol{\theta})=\mathbf{0}$ e devem obtidos numericamente maximizando (5.65).

\subsection{Seleção de modelos}

Para a seleção de modelos, propomos avaliar a série de ajustes de modelos inspecionando os critérios de informação usados no modelo PNLMM. Lembramos aqui os critérios considerados. O Critério de Informação de Akaike Marginal, $m A I C=-2 \ell(\widehat{\boldsymbol{\theta}} \mid \mathbf{Y})+2 p N /(N-p-1)$, em que $p$ é o número de parâmetros estimados no modelo considerado, e $N=\sum_{i=1}^{n} m_{i}$, (veja Vaida e Blanchard, 2005); o Critério de Informação Bayesiano de Schwarz, $B I C_{h}=-2 \ell(\widehat{\boldsymbol{\theta}}$ $\mathbf{Y})+p_{r} \log (n)+p_{f} \log (N)$, em que $p_{r}$ é o número de parâmetros correspondentes à componente aleatória, $p_{f}$ é o número de parâmetros correspondentes à componente fixa (veja Delandre et al. , 2014). Propomos também usar o Critério de Informação de Akaike modificado, usualmente chamado de AIC consistente, $C A I C=-2 \ell(\widehat{\boldsymbol{\theta}} \mid \mathbf{Y})+p(1+\log (N))$, e o Critério de Informaçãp de Hannan-Quinn, $H Q I C=-2 \ell(\widehat{\boldsymbol{\theta}} \mid \mathbf{Y})+p \log (\log (N))$, em que $p$ é o número de parâmetros estimados no modelo sendo considerado. Assim como no modelo PNLMM, o melhor modelo é aquele com o menor mAIC (ou $\mathrm{BIC}_{h}$ ou CAIC ou HQIC).

\subsection{Simulação}

Com o intuito de estudar as propriedades do estimador de máxima verossimilhança $\boldsymbol{\theta}=$ $\left(\boldsymbol{\beta}^{\top}, \boldsymbol{\theta}_{\epsilon}^{\top}, \boldsymbol{\theta}_{\gamma}^{\top}\right)^{\top}$, realizamos um estudo de simulação de Monte Carlo em que foi considerado o modelo linear misto

$$
Y_{i j}=\beta_{0}+\beta_{1} x_{i j}+\gamma_{i}+\epsilon_{i j}
$$

para $j=1, \ldots, 5$ e $i=1, \ldots, n$. Nós consideramos dois cenârios.

No primerio cenârio supomos que $\gamma_{i} \stackrel{\text { iid }}{\sim} \mathrm{PN}\left(0, \eta_{g}, \alpha_{g}\right)$, e $\epsilon_{i j} \stackrel{\text { ind }}{\sim} \mathrm{PN}\left(0, \eta_{e}, \alpha_{e}\right)$, portanto $\boldsymbol{\beta}=$ $\left(\beta_{0}, \beta_{1}\right)^{\top}, \boldsymbol{\theta}_{\epsilon}=\left(\eta_{e}, \alpha_{e}\right)^{\top}$ e $\boldsymbol{\theta}_{\alpha}=\left(\eta_{g}, \alpha_{g}\right)^{\top}$, em que os verdadeiros valores dos parâmetros foram tomados como $\beta_{0}=3, \beta_{1}=-1.5, \eta_{e}=\eta_{g}=1.50, \alpha_{e}=0.50,1.5$ e $\alpha_{g}=0.50$, 3.0. Tomamos esses valores de $\alpha$ para acomodar assimetria à esquerda (valores de $\alpha<1$ ) e à direita (valores de $\alpha>1)$.

No segundo cenârio supomos que $\gamma_{i} \stackrel{\text { iid }}{\sim} \mathrm{SN}\left(0, \eta_{g}, \lambda_{g}\right)$, e $\epsilon_{i j} \stackrel{\text { ind }}{\sim} \mathrm{SN}\left(0, \eta_{e}, \lambda_{e}\right)$, portanto $\boldsymbol{\beta}=$ $\left(\beta_{0}, \beta_{1}\right)^{\top}, \boldsymbol{\theta}_{\epsilon}=\left(\eta_{e}, \lambda_{e}\right)^{\top}$ e $\boldsymbol{\theta}_{\alpha}=\left(\eta_{g}, \lambda_{g}\right)^{\top}$, em que os verdadeiros valores dos parâmetros foram 
tomados como $\beta_{0}=3, \beta_{1}=-1.5, \eta_{e}=\eta_{g}=1.50, \lambda_{e}=-2.5,1.5$ e $\alpha_{g}=-1.5,1.5,2.5$. O valores de $\lambda$ foram assumidos para acomodar assimetria à esquerda (valores de $\lambda<0$ ) e à direita (valores de $\lambda>0$ ).

Nos dois cenârios descritos acima, a covariável $x_{i}$ foi gerada de acordo com uma distribuição $\mathrm{U}(0.1,20)$ e foram simulados 1000 conjuntos da dados de Monte Carlo de tamanhos $n=50,100$, 200,500 e 1000 do modelo (5.67). Consideramos também níveis de censura iguais a $p_{0}=10,15 \%$ (à esquerda), e $p_{2}=10,20 \%$ à direita.

Para estudar as propriedades assintóticas dos estimadores, consideramos o viés relativo (VR) em valor absoluto, e a raiz quadrada do erro quadrático médio (REQM) as quais são dados por

$$
\operatorname{VR}\left(\widehat{\theta}_{i}\right)=\frac{1}{1000} \sum_{k=1}^{1000}\left(\frac{\widehat{\theta}_{i}^{(k)}}{\theta_{i}}-1\right), \operatorname{REQM}\left(\widehat{\theta}_{i}\right)=\sqrt{\frac{1}{1000} \sum_{k=1}^{1000}\left(\widehat{\theta}_{i}^{(k)}-\theta_{i}\right)^{2}}
$$

respectivamente, em que $\widehat{\theta}_{i}^{(k)}$ é o estimador de $\theta_{i}$ para a $k$-ésima amostra, para $\theta_{i} \in \boldsymbol{\theta}=$ $\left(\boldsymbol{\beta}^{\top}, \boldsymbol{\theta}_{\epsilon}^{\top}, \boldsymbol{\theta}_{\gamma}^{\top}\right)^{\top}$. Os estimadores de máxima verossimilhança dos parâmetros foram computados usando a função optim do pacote estatístico R (R Development Core Team, 2017).

Das Tabelas A.3 e A.4 no Apêndice A pode-se observar que os estimadores dos parâmetros são estáveis. Também o VR e a REQM dos estimadores tendem se aproximar de zero quando o valor de $n$ aumenta indicando que os estimadores baseados no método de máxima verossimilhança fornecem boas propriedades assintóticas. Esse padrão é o mesmo para os diferentes valores de $\alpha_{e}$ e $\alpha_{g}$ sob consideração.

\subsection{Aplicações}

Nesta seção aplicamos os modelos estudados a dois conjuntos de dados. O primeiro deles são relacionados dados de doença periodontal e foram analisados previamente por Galvis et al. (2014) e Fernandes et al. (2006). O segundo conjunto de dados correspondem aos longitudinais estudo de colesterol em Framingham analisados no Capítulo 4.

\subsubsection{Dados de Doença Periodontal}

O objetivo desta aplicação é ilustrar o uso dos modelos PNDC-RE e SNDC-RE desenvolvidos anteriormente. O conjunto de dados usado nesta aplicação são relacionados a um estudo de periodontologia clínica. O nível de inserção clínica (NIC), um marcador clínico da doença periodontal (DP), foi registrado em cada um de 6 locais do dente por dente para 28 dentes. (considerado dentição total, excluindo os 4 terceiros molares). Com 290 sujeitos, o interesse foi quantificar a extensão e gravidade de DP para os tipos de dentes (4 caninos e 8 incisivos, pré-molares e molares). A variável resposta foi: "Proporção de locais doentes do dente, para cada um dos quatro tipos de dentes (com NIC $\geq 3 \mathrm{~mm}$ )". Isso dá origem a uma estrutura de dados em cluster em que cada sujeito registra 4 observações correspondentes aos 4 tipos de dentes. Os dentes perdidos foram considerados "perdidos devido à DP", em que todos os locais para esse dente contribuíram para a categoria doente. As covariáveis consideradas neste conjunto de dados incluem o sexo $(0=$ feminino; $1=$ masculino), idade do sujeito no momento do exame (em anos, variando de 26 a 87 anos $)$, indicador do status de hemoglobina glicosilada, HbA1c $(0=$ controlada, $<7 \%$; $1=$ não controlada $\geq 7 \%$ ) e indicador do status de Tabagismo ( $0=$ não fumante, $1=$ fumante). A categoria de fumante é composta tanto de fumantes atuais como passadas.

Para este análise, foram consideradas os tipos de dentes incisivos, pré-molares e molares a fim de ter um conjunto de dados com uma estrutura de assimetria. A categoria tipo de dente 


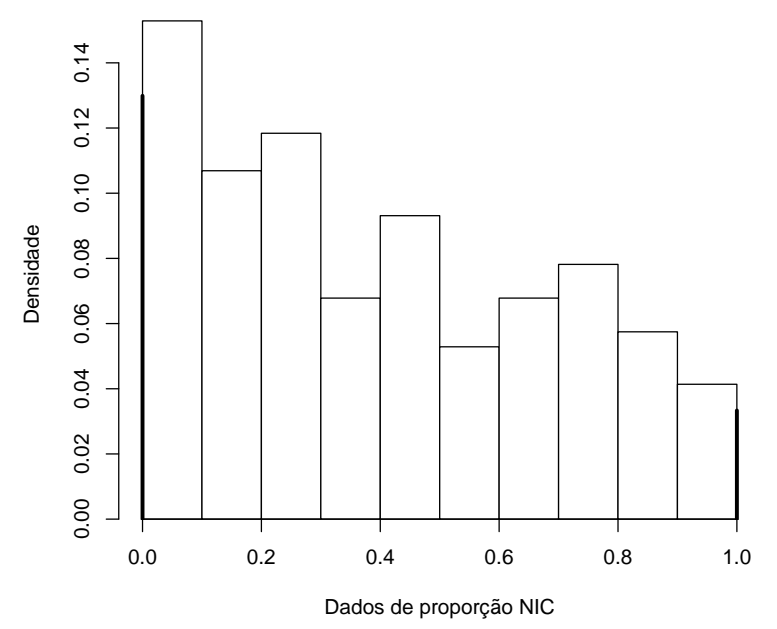

Figura 5.1: Histograma para os dados de doença periodontal. Os "pinos"nos extremos representam a proporção de observações censuradas $(9,8 \%)$ à esquerda e $(8,1 \%)$ à direita.

incisivos foi considerada como linha base. A Figura 5.1 apresenta o histograma para os dados da doença periodontal.

Para a análise dos dados foi adotado o seguinte modelo linear com intercepto aleatório:

$$
Y_{i j}=\beta_{0}+\beta_{1} \operatorname{sexo}_{i}+\beta_{2} \text { idade }_{i}+\beta_{3} \text { hba1c.d }+\beta_{4} \operatorname{premolar}_{i j}+\beta_{5} \operatorname{molar}_{i j}+\gamma_{i}+\epsilon_{i j}
$$

em que $Y_{i j}$ é a proporção de locais doentes do dente, para cada um dos três tipos de dentes considerados avaliado no $j$-ésimo tipo de dente $(j=1,2,3)$ para o sujeito $i(i=1, \ldots, 290)$.

Os seguintes modelos estatísticos, os quais diferem na distribuição dos erros e os efeitos aleatórios foram analisados

- Modelo 1: Um modelo com distribuição normal para os erros e distribuição normal-Potência para os efeitos aleatorios, isto é, $\epsilon_{i j} \stackrel{\text { iid }}{\sim} \mathrm{N}\left(0, \eta_{e}^{2}\right)$ e $\gamma_{i} \stackrel{\text { iid }}{\sim} \mathrm{PN}\left(0, \eta_{g}, \alpha_{g}\right)$; e que chamaremos ( modelo DCN-PN),

- Modelo 2: Um modelo com distribuição normal-potência para os erros e distribuição normal para os efeitos aleatorios, isto é, $\epsilon_{i j} \stackrel{\text { iid }}{\sim} \mathrm{PN}\left(0, \eta_{e}, \alpha_{e}\right)$ e $\gamma_{i} \stackrel{\text { iid }}{\sim} \mathrm{N}\left(0, \eta_{g}^{2}\right)$; e que chamaremos (modelo DCPN-N),

- Modelo 3: Um modelo com distribuição normal para os erros e distribuição normal-assimétrico para os efeitos aleatorios, isto é, $\epsilon_{i j} \stackrel{\text { iid }}{\sim} \mathrm{N}\left(0, \eta_{e}^{2}\right)$ e $\gamma_{i} \stackrel{\text { iid }}{\sim} \mathrm{SN}\left(0, \eta_{g}, \lambda_{g}\right)$; e que chamaremos (modelo $D C N-S N)$,

- Modelo 4: Um modelo com distribuição normal-assimétrico para os erros e distribuição normal para os efeitos aleatorios, isto é, $\epsilon_{i j} \stackrel{\text { iid }}{\sim} \mathrm{SN}\left(0, \eta_{e}, \lambda_{e}\right)$ e $\gamma_{i} \stackrel{\text { iid }}{\sim} \mathrm{N}\left(0, \eta_{g}^{2}\right)$; e que nós chamaremos (modelo DCSN-N),

- Modelo 5: Um modelo com distribuição normal para os erros e os efeitos aleatorios, isto é, $\epsilon_{i j} \stackrel{\mathrm{iid}}{\sim} \mathrm{N}\left(0, \eta_{e}^{2}\right)$ e $\gamma_{i} \stackrel{\mathrm{iid}}{\sim} \mathrm{N}\left(0, \eta_{g}^{2}\right)$; e que chamaremos (modelo $\left.D C N-N\right)$. 
As estimativas dos parâmetros (apenas para as variáveis significativas no modelo) foram obtidos usando a função optim no R (R Development Core Team, 2017), e são mostrados na Tabela 4.2. Para o ajuste dos modelos foram usados 25 pontos da quadratura de Gauss-Hermite.

Escolhimos esse número de pontos de quadratura porque o aumento de pontos contribuiu pouco à aproximação do logaritmo da função de log-verosimilhança, isto é, as estimativas se mantiveram no mesmo valor à medida que aumentamos o número de pontos da quadratura.

A Tabela 5.2 mostra os critérios de comparação dos modelos ajustados. Segundo a tabela, todos os criterios (mAIC, $\mathrm{BIC}_{h}$, CAIC e HQIC), idicam que o modelo 4 (modelo DCN-SN) parece proporcionar um melhor ajuste aos dados da doença periodontal em relação aos outros modelos considerados, apoiando a contenção de afastamento da normalidade do efeito aleatório.

A suposição de distribuição normal-potência para os interceptos aleatórios é testada com a hipótese

$$
H_{01}: \alpha_{g}=1 \text { versus } H_{11}: \alpha_{g} \neq 1,
$$

usando a estatística de razão de veossimilhança (RV)

$$
\Lambda_{1}=\frac{L_{D C N-N}(\widehat{\boldsymbol{\theta}})}{L_{D C N-P N}(\widehat{\boldsymbol{\theta}})}
$$

a qual sob os dados em consideração, leva a $-2 \log \left(\Lambda_{1}\right)=-2\left(\ell_{D C N-N}(\widehat{\boldsymbol{\theta}})-\ell_{D C N-P N}(\widehat{\boldsymbol{\theta}})\right)=$ $12.091, \operatorname{com} p-v a l u e=0.0005$, com forte indicação em contra a hipótese nula.

Similarmente, a suposição de distribuição normal-potência para os efeitos aleatórios, e normalidade dos erros é testada usando a hipótese

$$
H_{02}: \alpha_{e}=1 \quad \text { versus } \quad H_{12}: \alpha_{e} \neq 1,
$$

usando a estatística de RV

$$
\Lambda_{2}=\frac{L_{D C N-N}(\widehat{\boldsymbol{\theta}})}{L_{D C P N-N}(\widehat{\boldsymbol{\theta}})},
$$

que para os dados sob estudo leva a $-2 \log \left(\Lambda_{2}\right)=-2\left(\ell_{D C N-N}(\widehat{\boldsymbol{\theta}})-\ell_{D C P N-N}(\widehat{\boldsymbol{\theta}})\right)=1.6824$, com $p$-value $=0.1946059$, e portanto rejeitamos a hipótese nula de que os erros seguem uma distribuição normal-potência.

As hipóteses acima permitem concluir que os efeitos aleatórios seguem uma distribuição assimétrica, assim o modelo 1 parece ser um modelo que ajusta bem aos dados em relação ao modeo normal.

\subsubsection{Dados de Colesterol}

Consideramos agora os dados de colesterol do estudo de Framingham que foram usados no Capítulo 4 para ilustrar os modelos tobit normal-potência com efeitos aleatórios (PNT-RE) e tobit normal-assimétrico com efeitos aleatórios (SNT-RE). Vamos supor que a variável resposta $Y_{i j}$, nível de colesterol em $\mathrm{mg} / \mathrm{dL}$ (dividido por 100) avaliado no $j$-ésimo tempo $\left(j=1, \ldots, m_{i}\right)$ para o sujeito $i(i=1, \ldots, 200)$ apresenta censura no valor $c_{0}=1.82$. (Este valor foi determinado considerando que o $10 \%$ das observações menores foi censurada). Esclarecemos que esta aplicação tem com objetivo simplesmente ilustrar o uso dos modelos PNT-RE e SNT-RE.

A Figura 5.2 apresenta o histograma para os dados de colesterol com a presença de censura no valor $c_{0}=1.82$. Note-se claramente a presença de assimetria e parece adequado considerar um modelo com efeitos aleatórios assimetricos para analisar o conjunto de dados.

Baseados nos resultados obtidos na Seção 4.5 em que a variável sexo não foi significativa, 


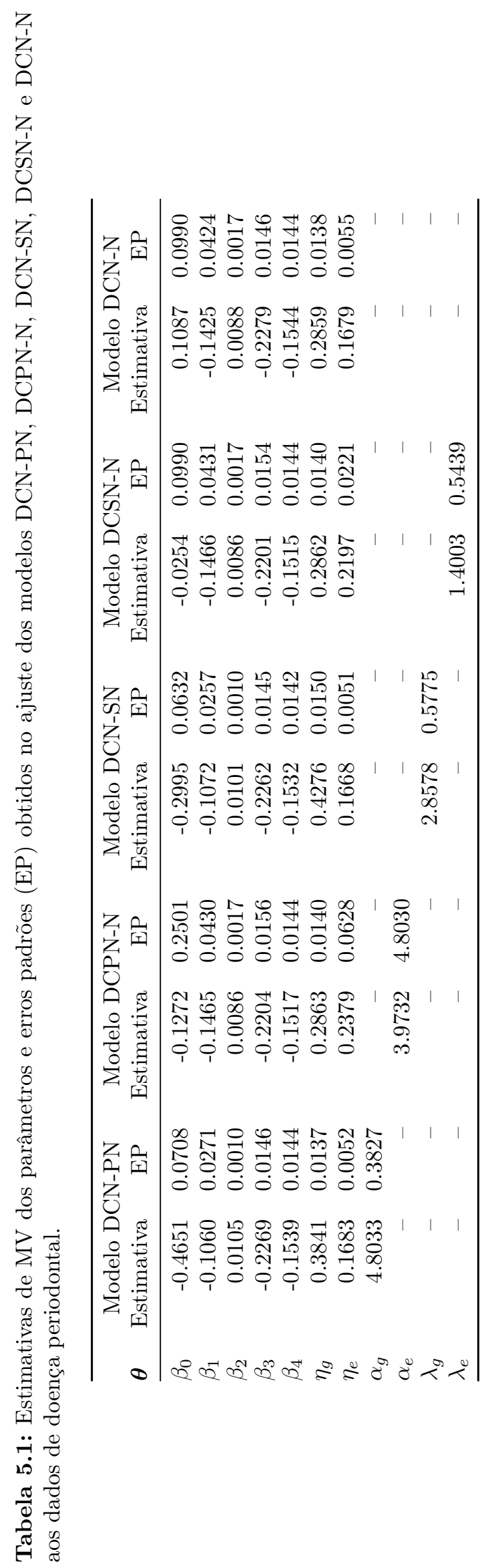


Tabela 5.2: Critérios de seleção dos modelos ajustados aos dados de doença periodontal.

\begin{tabular}{lccccc}
\hline Critério & Modelo DCN-PN & Modelo DCPN-N & Modelo DCN-SN & Modelo DCSN-N & Modelo DCN-N \\
\hline$\ell(\boldsymbol{\theta})$ & -139.9031 & -145.1074 & -132.8446 & -145.1626 & -145.9486 \\
mAIC & 295.9734 & 306.3820 & 281.8564 & 306.4924 & 306.0271 \\
BIC $_{h}$ & 331.7569 & 343.2641 & 317.6399 & 343.3745 & 338.1780 \\
CAIC & 341.9541 & 352.3627 & 327.8371 & 352.4731 & 346.2767 \\
HQIC & 333.9541 & 344.3627 & 319.8371 & 344.4731 & 339.2767 \\
\hline
\end{tabular}

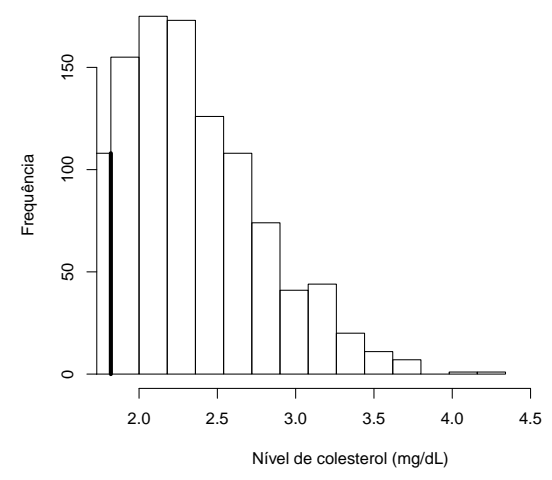

Figura 5.2: Histograma para os dados de Colesterol. O "pino"no extremo inferior representa a frequência de observações censuradas (108) no valor $c_{0}=1.82$.

adotamos o seguinte modelo linear misto:

$$
Y_{i j}=\beta_{0}+\beta_{1} \text { idade }_{i}+\beta_{2} t_{i j}+\gamma_{i}+\epsilon_{i j}
$$

sendo $i d a d e_{i}$ é a idade no inicio do estudo (em anos); e $t_{i j}$ é (tempo - 5)/10, com tempo medido em anos.

Os seguintes modelos estatísticos, os quais diferem na distribuição dos erros e os efeitos aleatórios foram considerados

- Modelo 1: Um modelo com distribuição normal para os erros e distribuição normal-Potência para os efeitos aleatorios, isto é, $\epsilon_{i j} \stackrel{\text { iid }}{\sim} \mathrm{N}\left(0, \eta_{e}^{2}\right)$ e $\gamma_{i} \stackrel{\text { iid }}{\sim} \mathrm{PN}\left(0, \eta_{g}, \alpha_{g}\right)$; e que chamaremos (modelo DCN-PN),

- Modelo 2: Um modelo com distribuição normal-potência para os erros e distribuição normal para os efeitos aleatorios, isto é, $\epsilon_{i j} \stackrel{\text { iid }}{\sim} \operatorname{PN}\left(0, \eta_{e}, \alpha_{e}\right)$ e $\gamma_{i} \stackrel{\text { iid }}{\sim} \mathrm{N}\left(0, \eta_{g}^{2}\right)$; e que chamaremos ( modelo DCPN-N),

- Modelo 3: Um modelo com distribuição normal para os erros e distribuição normal-assimétrico para os efeitos aleatorios, isto é, $\epsilon_{i j} \stackrel{\text { iid }}{\sim} \mathrm{N}\left(0, \eta_{e}^{2}\right)$ e $\gamma_{i} \stackrel{\text { iid }}{\sim} \mathrm{SN}\left(0, \eta_{g}, \lambda_{g}\right)$; e que chamaremos (modelo $D C N-S N)$,

- Modelo 4: Um modelo com distribuição normal-assimétrico para os erros e distribuição normal para os efeitos aleatorios, isto é, $\epsilon_{i j} \stackrel{\text { iid }}{\sim} \mathrm{SN}\left(0, \eta_{e}, \lambda_{e}\right)$ e $\gamma_{i} \stackrel{\text { iid }}{\sim} \mathrm{N}\left(0, \eta_{g}^{2}\right)$; e que chamaremos (modelo DCSN-N), 
- Modelo 5: Um modelo com distribuição normal para os erros e os efeitos aleatorios, isto é, $\epsilon_{i j} \stackrel{\mathrm{iid}}{\sim} \mathrm{N}\left(0, \eta_{e}^{2}\right)$ e $\gamma_{i} \stackrel{\text { iid }}{\sim} \mathrm{N}\left(0, \eta_{g}^{2}\right)$; e que chamaremos (modelo $\left.D C N-N\right)$.

As estimativas dos parâmetros (apenas para as variáveis significativas no modelo) foram obtidos usando a função optim no R (R Development Core Team, 2017), e são mostrados na Tabela 4.2. Para o ajuste dos modelos foram usados 23 pontos da quadratura de Gauss-Hermite. Escolhimos esse número de pontos de quadratura porque o aumento de pontos contribuiu pouco à aproximação do logaritmo da função de log-verosimilhança, isto é, as estimativas se mantiveram no mesmo valor à medida que aumentamos o número de pontos da quadratura.

A Tabela 5.2 mostra os critérios de comparação dos modelos ajustados. Segundo a tabela, todos os criterios (mAIC, BIC ${ }_{h}$, CAIC e HQIC), idicam que o modelo 4 (modelo DCN-PN) parece proporcionar um melhor ajuste aos dados de colesterol em relação aos outros modelos considerados, apoiando a contenção de afastamento da normalidade do efeito aleatório.

A suposição de distribuição normal-potência para os interceptos aleatórios é testada com a hipótese

$$
H_{01}: \alpha_{g}=1 \text { versus } H_{11}: \alpha_{g} \neq 1
$$

usando a estatística de razão de veossimilhança (RV)

$$
\Lambda_{1}=\frac{L_{D C N-N}(\widehat{\boldsymbol{\theta}})}{L_{N-P N}(\widehat{\boldsymbol{\theta}})},
$$

a qual sob os dados em consideração, leva a $-2 \log \left(\Lambda_{1}\right)=-2\left(\ell_{D C N-N}(\widehat{\boldsymbol{\theta}})-\ell_{D C N-P N}(\widehat{\boldsymbol{\theta}})\right)=$ 21.8808 , com $p-$ value $<0.0005$, com forte indicação em contra a hipótese nula.

Similarmente, a suposição de distribuição normal-potência para os efeitos aleatórios, e normalidade dos erros é testada usando a hipótese

$$
H_{02}: \alpha_{e}=1 \quad \text { versus } \quad H_{12}: \alpha_{e} \neq 1,
$$

usando a estatística de RV

$$
\Lambda_{2}=\frac{L_{D C N-N}(\widehat{\boldsymbol{\theta}})}{L_{D C P N-N}(\widehat{\boldsymbol{\theta}})},
$$

que para os dados sob estudo leva a $-2 \log \left(\Lambda_{2}\right)=-2\left(\ell_{D C N-N}(\widehat{\boldsymbol{\theta}})-\ell_{D C P N-N}(\widehat{\boldsymbol{\theta}})\right)=11.4112$, $\operatorname{com} p-$ value $=0.00073$, com forte indicação em contra a hipótese nula. Podes-se concluir que os efeitos aleatórios seguem uma distribuição normal-potência, assim o modelo 1 parece ser um modelo que ajusta bem aos dados em relação ao modelos DCN-SN e DCSN-N.

\subsection{Conclusões}

Neste capítulo, introduzimos um modelo de regressão linear misto que permite modelar dados que apresentam censura (à esquerda ou à direita ou ambos) ou que estão limitados em um intervalo de valores; e possuem coeficiente de assimetria e curtose maior ou menor do que pode ser modelado pelo modelo normal. Em particular, propomos as distribuições normal-potência (Durrans, 1992) e normal-assimétrica (Azzalini, 1985) para dar flexibilidade ao termo do erro e ao efeito aleatório em um modelo linear misto.

Mesmo como no Capítulo 3, a vantagem de considerar as distribuições PN e SN é que podem oferecer flexibilidade na captura de uma ampla gama de comportamentos não normais, incluindo formas assimétricas à esquerda e à direita, tendo a normalidade como caso especial (no caso do modelo PN). Derivamos os dois primeiros momentos da variável resposta e propomos um método 


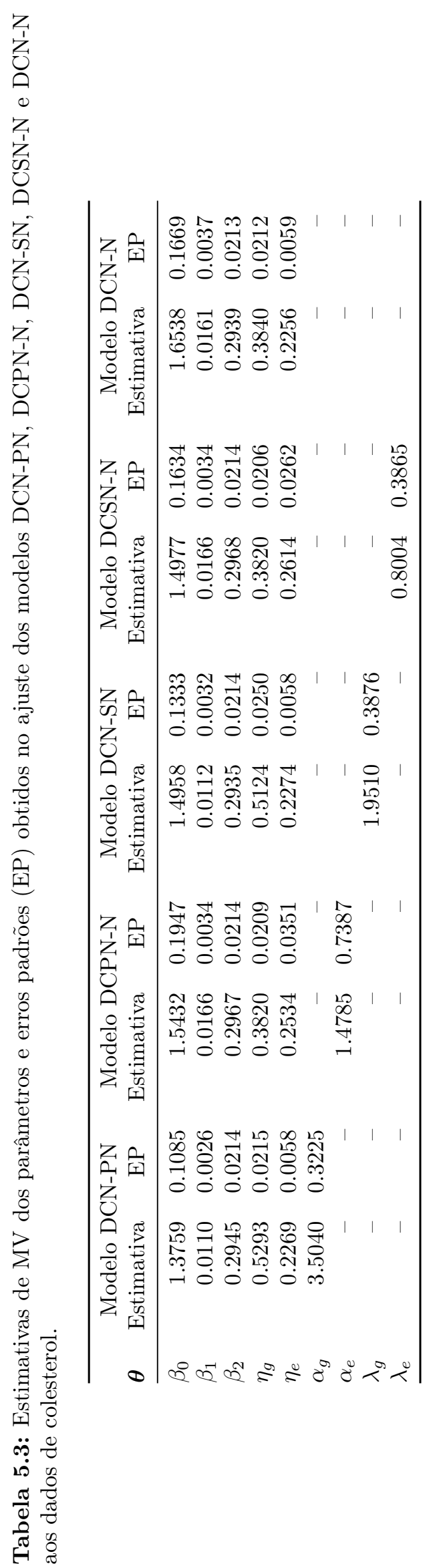


Tabela 5.4: Critérios de seleção dos modelos ajustados aos dados de colesterol.

\begin{tabular}{lccccc}
\hline Critério & Modelo DCN-PN & Modelo DCPN-N & Modelo DCDCN-SN & Modelo DCSN-N & Modelo DCN-N \\
\hline$\ell(\boldsymbol{\theta})$ & -270.7423 & -275.9771 & -271.6029 & -275.9851 & -281.6827 \\
mAIC & 553.5656 & 564.0352 & 555.2868 & 564.0512 & 573.4232 \\
BIC $_{h}$ & 579.8845 & 592.0066 & 581.6057 & 592.0226 & 596.4670 \\
CAIC & 589.1895 & 599.6591 & 590.9107 & 599.6751 & 603.1195 \\
HQIC & 583.1895 & 593.6591 & 584.9107 & 593.6751 & 598.1195 \\
\hline
\end{tabular}

de estimação dos parâmetros do modelo. As estimativas desses parâmetros são obtidas usando a abordagen de máxima verossimilhança com métodos de integração numérica (quadratura de Gauss-Hermite) que também são usados para estudar propriedades assintóticas dos estimadores.

Com casos particulares dos modelos considerados temos os modelos tobit normal-potência com efeitos aleatórios (PNT-RE), e modelo tobit normal-assimétrico com efeitos aleatórios (PNT$\mathrm{RE}$ ), os quais estendem o modelo Tobit usual (Tobin, 1958) com interceptos aleatórios normais

Apresentamos um exemplo de motivação para ilustrar a nossa proposta e concluímos que podem ser alternativas úteis a outros métodos existentes na literatura como o modelo linear beta misto por Figueroa-Zúñiga et al. (2013). Então, acreditamos que o modelo proposto neste capítulo amplia a opção na classe de modelos lineares mistos, para ajustar dados particularmente assimétricos com indicação de distribuição não normal para o efeito aleatório. 


\section{Capítulo 6}

\section{Conclusões Finais e Perspectivas Futuras}

\subsection{Conclusões Finais}

Neste trabalho estudamos algumas extensões dos modelos potência-alfa (Gupta e Gupta , 2008). Inicialmante estudamos extensões do modelo PT às situações em que a variável resposta apresenta censura ou truncamento além de ter assimetria e/ou curtose maior do que o modelo normal usual pode ajustar. O modelo é denotado por CR-PT é uma alternativa para os modelos de regressão $t$ de Student censurado (Arellano-Valle et al., 2012) e os modelos sobre a classe de distribuições SNM (Garay et al., 2017), sendo capaz de modelar dados com caudas mais pesadas do que os modelos tobit normal e tobit $t$-Student usual.

No trabalho também estudamos extensões do modelo normal-potência para às situações dos modelos con efeitos aleatórios (modelos lineares mistos) quando os dados apresentam dupla censura (ou censura à esquera ou à direita). Como casos particulares encontra-se extensões do modelos tobit normal usual com efeitos aleatórios, denotados PNT-RE e SNT-RE (sob a suposição de que os erros e os efeitos aleatórios seguem una distrubuição normal-assimétrica).

A inferência estatística dos modelos estudados neste trabalho é realizada desde a abordagem clássica e é baseada no método da máxima verossimilhança. Para a obtenção de estimativas dos parâmetros dos modelos estudados é nessário o uso do método de integração numérica de Gauss-Hermite para aproximar as integrais envolvidas na função de log-veroossimilhança.

Para uma grande parte dos modelos estudados foram realizados estudos de simulação a fim de estudar as suas bondades e limitações. Os resultados das simulações mostram boas propriedades assintóticas dos estimadores obtidas pelo método.

Aplicações com dados reales sob altitudes de vulcões do mundo, salário de mulheres casadas, níveis de colesterol e doença periodontal mostram que os modelos estudados podem ser alternativas úteis para a modelagem de dados com a características consideradas (grau de assimetria e curtose maior do que o modelo normal usual, por exemplo)

Entre as principais bondades dos modelos estudados, resaltamos que podem oferecer flexibilidade na captura de uma ampla gama de comportamentos não normais, incluindo formas assimétricas à esquerda e à direita, tendo a normalidade como caso especial. Os modelos são também úteis para modelar dados com caudas ainda mais pesadas do que alguns modelos existentes na literatura. O uso de estatísticas baseados na razão de verosimilhanças no caso dos modelos sob a suposição de distribuição normal-potência, é também uma das bondades dos modelos, por exemplo a hipótese assimetria dos erros e o termo do erro nos modelos com efeitos aleatórios podem ser raealizadas.

Consideramos que os modelos estudados constituem uma alternativa viável para a modelagem 
de dados longitudinais o em cluster cuando a variável resposta apresenta assimetia e censura.

\subsection{Perspectivas Futuras}

Varios trabalhos de pesquisa podem ser derivados e/ou direcionados a partir dos resultados deste trabalho, entre eles podemos mencionar os seguintes:

- Estudar a inferência estatística do modelo de regressão linear t-potência censurado.

- Explorar a metodologia de analise dos residuos e métodos diagnósticos para os modelos PNDC-RE, PNT-RE, SNDC-RE e PNT-RE desenvolvidos no Capítulo 5.

- Propor a inferência estatítica dos modelos lineares mistos normal-potência (PNLMM) estudados no Capítulo 4 desde uma perspectiva Bayesiana.

- Considerar a abordagem Bayesiana para fazer a inferéncia estatística dos modelos de regressão lineares mistos assimétricos para dados duplamente censurados (PNDC-RE e SNDC$\mathrm{RE})$, que foram estudados no Capítulo 5.

- Considerar a distribuição t-potência estudada por Zhao e Kim (2016) como modelo alternativo aos modelos estudados neste trabalo e realizar o estudo desde uma perspectiva clássica e Bayesiana.

- Criar um pacote estatístico que permita ajustar os modelos estudados e para disponibilizar no R para su uso livre. 


\section{Apêndice A}

\section{Tabelas das Simulações}

A seguir apresentamos as tabelas dos estudos de simulação feitos para os distintos modelos estudados. 


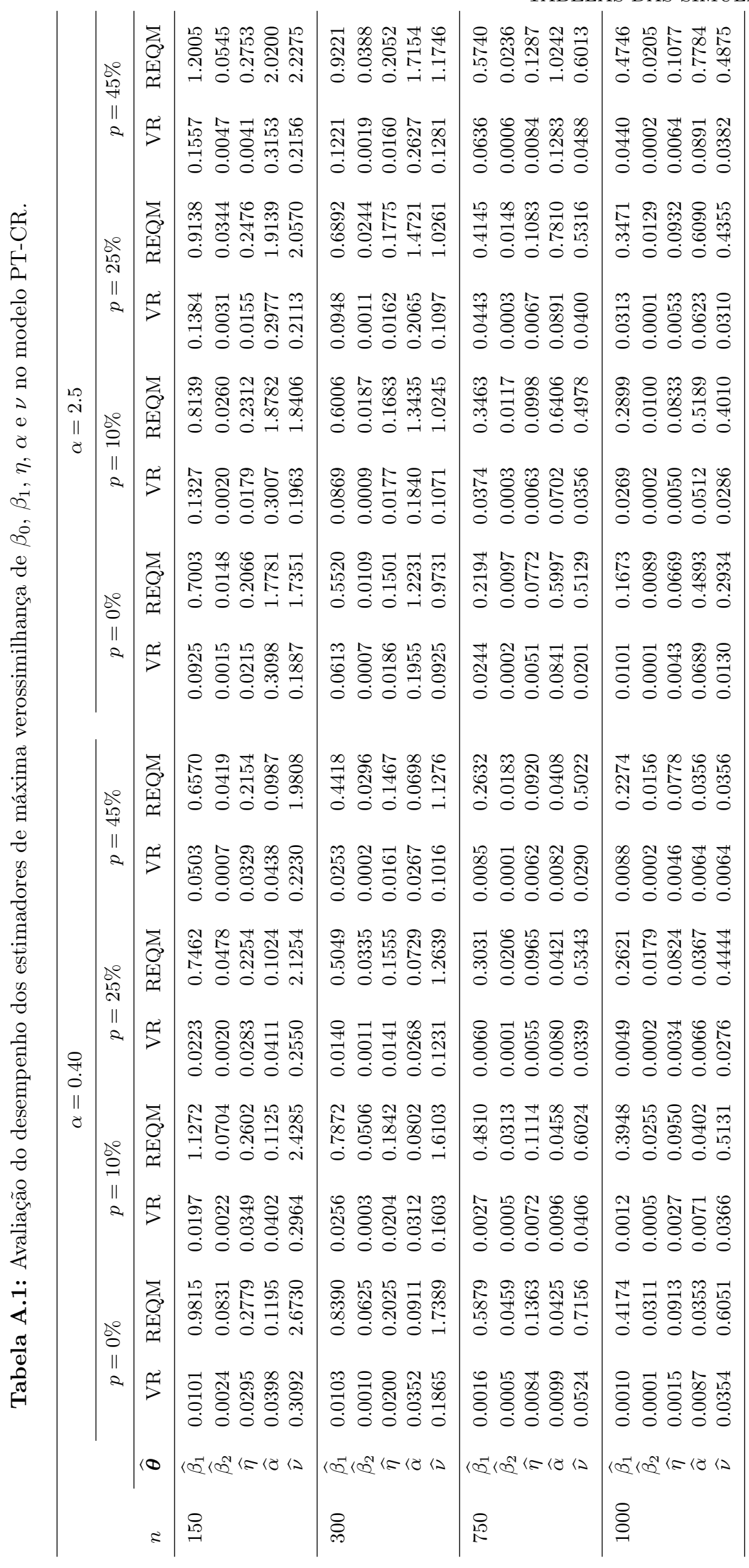




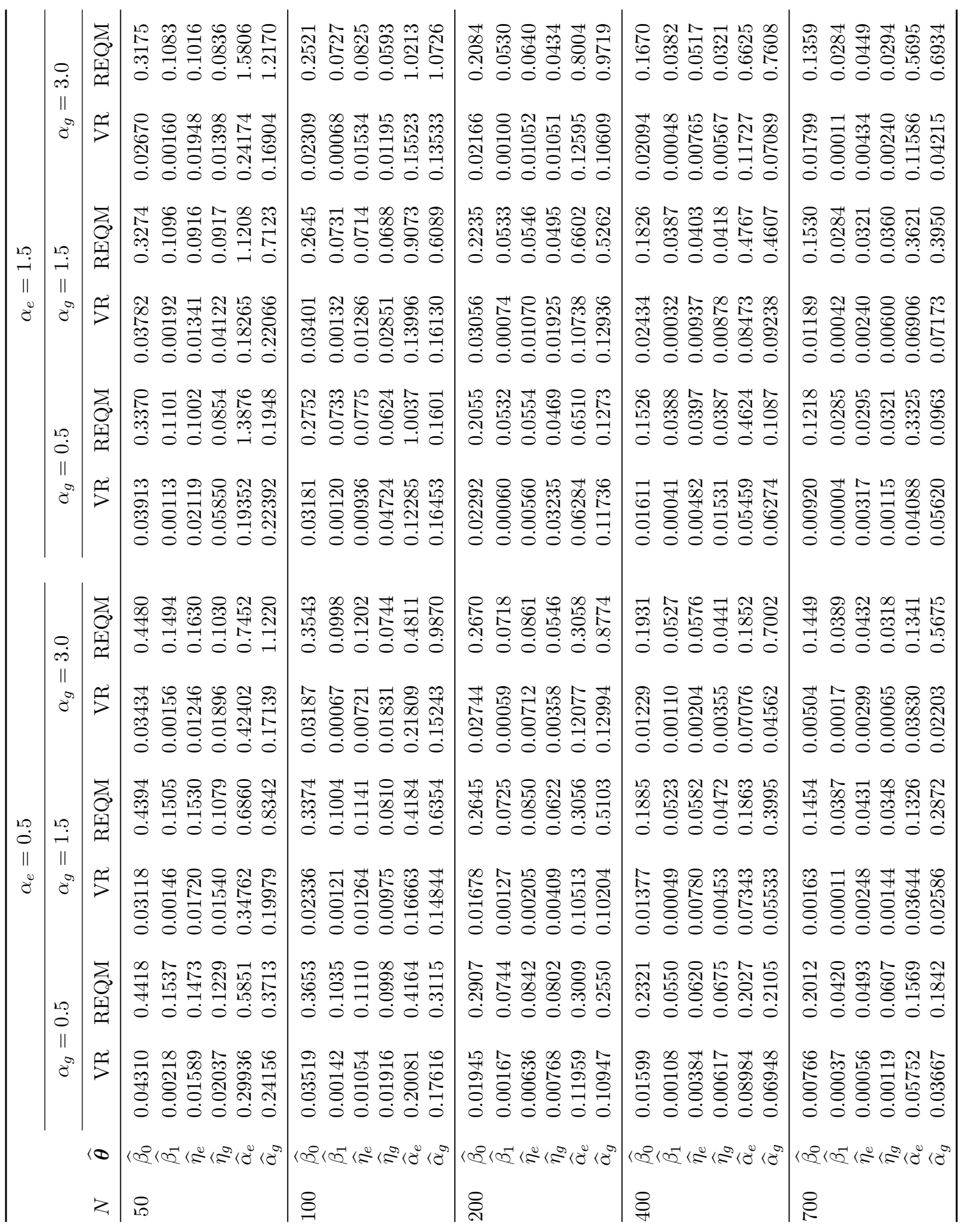




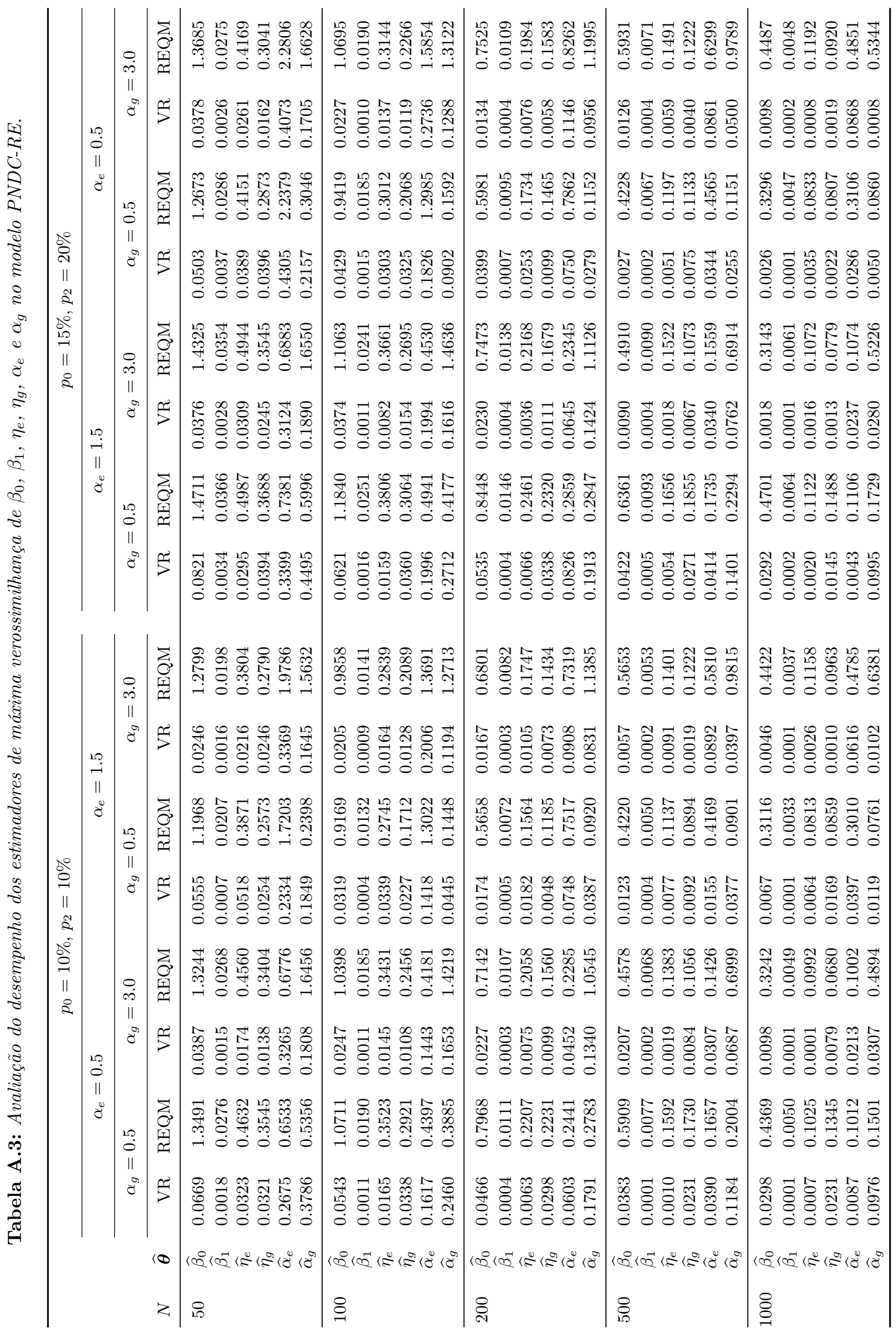




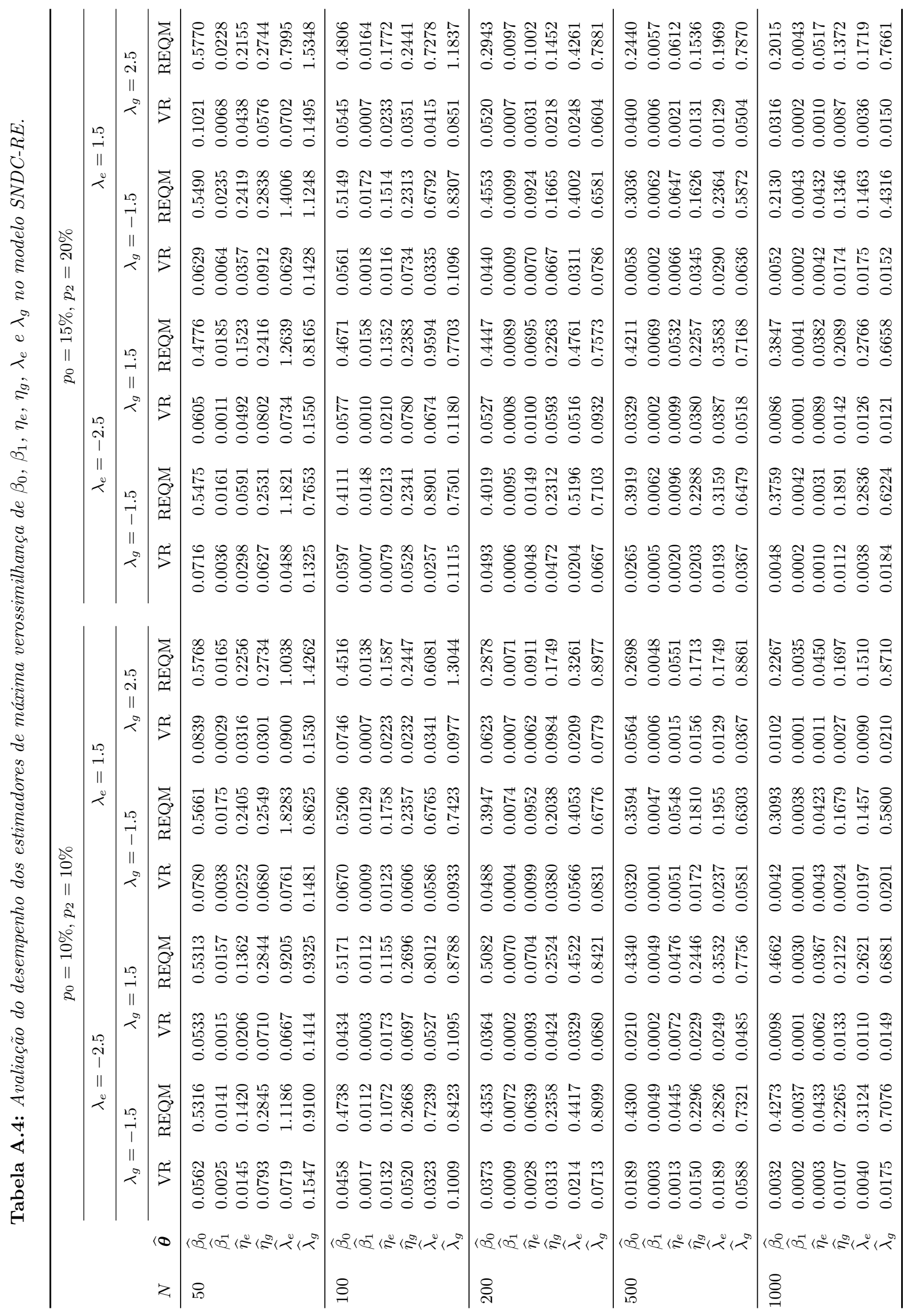




\section{Apêndice B}

\section{Funções Escore e Matrizes de Informação.}

\section{B.1 Matriz de Informação para o Modelo PST}

Apresentamos nesta seção as expressões para elementos da matriz de informação observada do modelo PST nas versões padronizada e de locação-escala desenvolvidos na Seção 3.2.

\section{B.1.1 Modelo PST Padronizado}

Computando a segunda derivada parcial da função de log-verossimilhança (3.5) e multiplicando por -1, obtemos os elementos da matriz de informação observada do modelo PST padronizado. Inicialmente definimos $w=\sqrt{\frac{\nu+1}{x^{2}+\nu}}, r(x \mid \nu)=t(x \mid \nu) / T(x \mid \nu), r_{\lambda}(x \mid \nu)=f_{S T}(x \mid$ $\lambda, \nu) / F_{S T}(x \mid \lambda, \nu)$ e $R_{\lambda}(x \mid \nu)=\left(1+\left(1+\lambda^{2}\right) x^{2} / \nu\right)^{-\frac{\nu}{2}} / F_{S T}(x \mid \lambda, \nu)$, assim, denotando elementos da matriz de informação observada do modelo PST como $j_{\lambda \lambda}, j_{\lambda \alpha}, \ldots, j_{\alpha \nu}, j_{\nu \nu}$, e depois algumas manipulações algebraicas, segue-se que

$$
\begin{aligned}
j_{\lambda \lambda}= & \frac{\lambda(\nu+2)}{\nu} \sum_{i=1}^{n} x_{i}^{3} w_{i}\left(1+\left(1+\lambda^{2}\right) \frac{x_{i}^{2}}{\nu}\right)^{-1} r\left(\lambda x_{i} w_{i} \mid \nu+1\right) \\
& +\frac{\nu+1}{\nu} \sum_{i=1}^{n} x_{i}\left(1+\frac{x_{i}^{2}}{\nu}\right)^{-1}\left[r\left(\lambda x_{i} w_{i} \mid \nu+1\right)\right]^{2} \\
& -\frac{2(\alpha-1)}{\pi} \frac{\lambda}{\left(1+\lambda^{2}\right)^{2}} \sum_{i=1}^{n}\left(1+\left(1+\lambda^{2}\right) \frac{x_{i}^{2}}{\nu}\right)^{-1} R_{\lambda}\left(x_{i} \mid \nu\right) \\
& -\frac{\alpha-1}{\pi} \frac{\lambda}{1+\lambda^{2}} \frac{\nu+2}{\nu} \sum_{i=1}^{n} x_{i}^{2}\left(1+\left(1+\lambda^{2}\right) \frac{x_{i}^{2}}{\nu}\right)^{-1} R_{\lambda}\left(x_{i} \mid \nu\right) \\
& +\frac{\alpha-1}{\pi^{2}\left(1+\lambda^{2}\right)^{2}} \sum_{i=1}^{n}\left[R_{\lambda}\left(x_{i} \mid \nu\right)\right]^{2} \\
j_{\lambda \alpha}= & \frac{1}{\pi\left(1+\lambda^{2}\right)} \sum_{i=1}^{n} R_{\lambda}\left(x_{i} \mid \nu\right)
\end{aligned}
$$




$$
\begin{aligned}
j_{\lambda \nu}= & -\frac{1}{2 \nu(\nu+1)} \sum_{i=1}^{n} x_{i}\left(x_{i}^{2}-1\right) w_{i}\left(1+\frac{x_{i}^{2}}{\nu}\right)^{-1} r\left(\lambda x_{i} w_{i} \mid \nu+1\right) \\
& +\frac{\lambda^{2}}{2 \nu^{2}} \frac{\nu+2}{(\nu+1)^{2}} \sum_{i=1}^{n} x_{i}^{3}\left(x_{i}^{2}-1\right) w_{i}\left(1+\frac{x_{i}^{2}}{\nu}\right)^{-1}\left(1+\left(1+\lambda^{2}\right) \frac{x_{i}^{2}}{\nu}\right)^{-1} r\left(\lambda x_{i} w_{i} \mid \nu+1\right) \\
& +\frac{\lambda}{2 \nu^{2}} \sum_{i=1}^{n} x_{i}^{2}\left(x_{i}^{2}-1\right)\left(1+\frac{x_{i}^{2}}{\nu}\right)^{-2}\left[r\left(\lambda x_{i} w_{i} \mid \nu+1\right)\right]^{2} \\
& +\frac{\alpha-1}{2 \pi(\nu+1)} \frac{1-\lambda^{2}}{\left(1+\lambda^{2}\right)^{2}} \sum_{i=1}^{n} R_{\lambda}\left(x_{i} \mid \nu\right)+\frac{\alpha-1}{2 \pi(\nu+1)} \frac{\lambda}{\left(1+\lambda^{2}\right)^{2}} \sum_{i=1}^{n}\left[R_{\lambda}\left(x_{i} \mid \nu\right)\right]^{2} \\
& -\frac{\alpha-1}{2 \pi(\nu+1)} \frac{\lambda^{2}}{1+\lambda^{2}} \sum_{i=1}^{n} x_{i}^{2}\left(1+\left(1+\lambda^{2}\right) \frac{x_{i}^{2}}{\nu}\right)^{-1} R_{\lambda}\left(x_{i} \mid \nu\right) \\
& -\frac{\alpha-1}{2 \pi\left(1+\lambda^{2}\right)} \sum_{i=1}^{n} \frac{g\left(x_{i}, \nu\right)}{F_{S T}\left(x_{i} \mid \lambda, \nu\right)} R_{\lambda}\left(x_{i} \mid \nu\right) \\
& -\frac{\alpha-1}{2 \pi} \sum_{i=1}^{n} \frac{g_{1}\left(x_{i}, \nu\right)}{F_{S T}\left(x_{i} \mid \lambda, \nu\right)} \\
& +\frac{1}{2 \pi(\nu+1)} \frac{\lambda}{1+\lambda^{2}} \sum_{i=1}^{n} R_{\lambda}\left(x_{i} \mid \nu\right)-\frac{1}{2} \sum_{i=1} \frac{g\left(x_{i}, \nu\right)}{F_{S T}\left(x_{i} \mid \lambda, \nu\right)} \\
j_{\alpha \alpha}= & \frac{n}{\alpha^{2}} \\
j_{\alpha \nu}= & -\frac{n}{2} \psi\left(\frac{\nu+1}{2}\right)+\frac{n}{2} \psi\left(\frac{\nu}{2}\right)+\frac{n}{2 \nu} \\
& \\
& \\
&
\end{aligned}
$$




$$
\begin{aligned}
& j_{\nu \nu}=-\frac{n \alpha}{2 \nu^{2}}-\frac{n \alpha}{4} \psi_{1}\left(\frac{\nu+1}{2}\right)+\frac{n \alpha}{4} \psi_{1}\left(\frac{\nu}{2}\right)-\frac{\nu-1}{2 \nu^{3}} \sum_{i=1}^{n} x_{i}^{2}\left(1+\frac{x_{i}^{2}}{\nu}\right)^{-1} \\
& +\frac{\nu+1}{2 \nu^{3}} \sum_{i=1}^{n} x_{i}^{2}\left(1+\frac{x_{i}^{2}}{\nu}\right)^{-2} \\
& +\frac{\lambda}{4(\nu+1)^{2}} \frac{1}{\nu^{2}} \sum_{i=1}^{n} x_{i}\left(x_{i}^{2}-1\right)\left(x_{i}^{2}+4 \nu+3\right) w_{i}\left(1+\frac{x_{i}^{2}}{\nu}\right)^{-2} r\left(\lambda x_{i} w_{i} \mid \nu+1\right) \\
& -\frac{\lambda}{4 \nu(\nu+1)}\left(\psi\left(\frac{\nu+2}{2}\right)-\psi\left(\frac{\nu+1}{2}\right)-\frac{1}{\nu+1}\right) \\
& \sum_{i=1}^{n} x_{i}\left(x_{i}^{2}-1\right) w_{i}\left(1+\frac{x_{i}^{2}}{\nu}\right)^{-1} r\left(\lambda x_{i} w_{i} \mid \nu+1\right) \\
& -\frac{\lambda^{3}}{4(\nu+1)} \frac{\nu+2}{\nu^{3}} \sum_{i=1}^{n} x_{i}^{3}\left(x_{i}^{2}-1\right)\left(1+\frac{x_{i}^{2}}{\nu}\right)^{-2}\left(1+\left(1+\lambda^{2}\right) \frac{x_{i}^{2}}{\nu}\right)^{-1} r\left(\lambda x_{i} w_{i} \mid \nu+1\right) \\
& +\frac{\lambda}{4 \nu(\nu+1)} \sum_{i=1}^{n} x_{i}\left(x_{i}^{2}-1\right) \ln \left(1+\frac{\lambda^{2} x_{i}^{2}}{\nu+x_{i}^{2}}\right) r\left(\lambda x_{i} w_{i} \mid \nu+1\right) \\
& +\frac{\lambda^{2}}{4 \nu^{3}(\nu+1)} \sum_{i=1}^{n} x_{i}^{2}\left(x_{i}^{2}-1\right)^{2}\left(1+\frac{x_{i}^{2}}{\nu}\right)^{-3}\left[r\left(\lambda x_{i} w_{i} \mid \nu+1\right)\right]^{2} \\
& -\frac{\alpha-1}{2 \pi(\nu+1)^{2}} \frac{\lambda}{1+\lambda^{2}} \sum_{i=1}^{n} R_{\lambda}\left(x_{i} \mid \nu\right) \\
& +\frac{\alpha-1}{4 \pi(\nu+1)} \frac{\lambda}{\nu} \sum_{i=1}^{n} x_{i}^{2}\left(1+\left(1+\lambda^{2}\right) \frac{x_{i}^{2}}{\nu}\right)^{-1} R_{\lambda}\left(x_{i} \mid \nu\right) \\
& -\frac{\alpha-1}{4 \pi(\nu+1)} \frac{\lambda}{1+\lambda^{2}} \sum_{i=1}^{n} \ln \left(1+\left(1+\lambda^{2}\right) \frac{x_{i}^{2}}{\nu}\right) R_{\lambda}\left(x_{i} \mid \nu\right) \\
& +\frac{\alpha-1}{4 \pi(\nu+1)} \frac{\lambda}{1+\lambda^{2}}\left(\psi\left(\frac{\nu+1}{2}\right)-\psi\left(\frac{\nu}{2}\right)-\frac{1}{\nu}\right) \sum_{i=1}^{n} R_{\lambda}\left(x_{i} \mid \nu\right) \\
& -\frac{\alpha-1}{2 \pi(\nu+1)} \frac{\lambda}{1+\lambda^{2}} \sum_{i=1}^{n} \frac{g\left(x_{i}, \nu\right)}{F_{S T}\left(x_{i} \mid \lambda, \nu\right)} R_{\lambda}\left(x_{i} \mid \nu\right) \\
& +\frac{\alpha-1}{4 \pi^{2}(\nu+1)^{2}} \frac{\lambda^{2}}{\left(1+\lambda^{2}\right)^{2}} \sum_{i=1}^{n}\left[R_{\lambda}\left(x_{i} \mid \nu\right)\right]^{2} \\
& +\frac{\alpha-1}{4} \sum_{i=1}^{n}\left(\frac{g\left(x_{i}, \nu\right)}{F_{S T}\left(x_{i} \mid \lambda, \nu\right)}\right)^{2}-\frac{\alpha-1}{2} \sum_{i=1}^{n} \frac{g_{2}\left(x_{i}, \nu\right)}{F_{S T}\left(x_{i} \mid \lambda, \nu\right)}
\end{aligned}
$$

em que $\psi(x)=\frac{d}{d x} \log \Gamma(x)$ e $\psi_{1}(x)=\frac{d}{d x} \psi(x)$ são as funções digama e trigama respectiva- 
mente, $g(x ; \nu)$ é dado em $(3.9)$ e

$$
\begin{aligned}
& g_{1}(x ; \nu)=\int_{-\infty}^{x}\left\{\frac{(\nu+1) s^{2}}{\nu\left(s^{2}+\nu\right)}-\log \left(1+\frac{s^{2}}{\nu}\right)\right\}\left\{1+\frac{\left(1+\lambda^{2}\right) s^{2}}{\nu}\right\}^{-\frac{\nu+2}{2}} s d s \\
&-\int_{-\infty}^{x} \frac{s}{s^{2}+\nu}\left\{1+\frac{\left(1+\lambda^{2}\right) s^{2}}{\nu}\right\}^{-\frac{\nu+2}{2}} d s \\
&+\int_{-\infty}^{x} \frac{\lambda^{2}(\nu+2) s^{3}}{\left(s^{2}+\nu\right)\left(\nu+\left(1+\lambda^{2}\right) s^{2}\right)}\left\{1+\frac{\left(1+\lambda^{2}\right) s^{2}}{\nu}\right\}^{-\frac{\nu+2}{2}} d s \\
& g_{2}(x ; \nu)=\int_{-\infty}^{x}\left\{\frac{s^{2}\left(s^{2} \nu-2 \nu-s^{2}\right)}{\nu^{2}\left(s^{2}+\nu\right)^{2}}+\frac{1}{2}\left[\frac{(\nu+1) s^{2}}{\nu\left(s^{2}+\nu\right)}-\log \left(1+\frac{s^{2}}{\nu}\right)\right]^{2}\right\} f_{S T}(s \mid \lambda, \nu) d s \\
&+\frac{\lambda}{2 \pi(\nu+1)} \int_{-\infty}^{x} \frac{s\left(s^{2}-1\right)}{\left(s^{2}+\nu\right)}\left\{\frac{(\nu+1) s^{2}}{\nu\left(s^{2}+\nu\right)}-\log \left(1+\frac{s^{2}}{\nu}\right)\right\} \\
& \times\left\{1+\frac{\left(1+\lambda^{2}\right) s^{2}}{\nu}\right\} \frac{\nu+2}{2} d s \\
& \frac{\lambda}{\pi} \int_{-\infty}^{x} \frac{s}{(s+\nu)^{2}}\left\{1+\frac{\left(1+\lambda^{2}\right) s^{2}}{\nu}\right\}^{-\frac{\nu+2}{2}} d s \\
&+ \frac{\lambda}{2 \pi}\left(\psi\left(\frac{\nu+1}{2}\right)-\psi\left(\frac{\nu}{2}\right)-\frac{1}{\nu}\right) \int_{-\infty}^{x} \frac{s}{s^{2}+\nu}\left\{1+\frac{\left(1+\lambda^{2}\right) s^{2}}{\nu}\right\} \\
&-\frac{\lambda}{2 \pi} \int_{-\infty}^{x} \frac{s}{s^{2}+\nu}\left\{\frac{(\nu+2)\left(1+\lambda^{2}\right) s^{2}}{\nu\left(\nu+\left(1+\lambda^{2}\right) s^{2}\right)}-\log \left(1+\frac{\left(1+\lambda^{2}\right) s^{2}}{\nu}\right)\right\} \\
& \times\left\{1+\frac{\left(1+\lambda^{2}\right) s^{2}}{\nu}\right\}^{-\frac{\nu+2}{2}} d s \\
& d s
\end{aligned}
$$

\section{B.1.2 Modelo PST de Locação-Escala}

Procedendo como no modelo PST padronizado, os elementos da matriz de informação observada para o modelo PST de locação-escala são obtidos computando a segunda derivada da 
função de log-verossimilhança em (3.15), e são dado por

$$
\begin{aligned}
& j_{\xi \xi}=-\frac{1}{\eta^{2}} \frac{\nu+1}{\nu^{2}} \sum_{i=1}^{n} z_{i}^{2}\left(1+\frac{z_{i}^{2}}{\nu}\right)^{-2}+\frac{1}{\eta^{2}} \frac{\nu+1}{\nu} \sum_{i=1}^{n}\left(1+\frac{z_{i}^{2}}{\nu}\right)^{-2} \\
& +\frac{\lambda}{\eta^{2}} \sum_{i=1}^{n} z_{i} w_{i}\left(1+\frac{z_{i}^{2}}{\nu}\right)^{-1} r\left(\lambda z_{i} w_{i} \mid \nu+1\right) \\
& +\frac{2}{\eta^{2}} \frac{\lambda}{\nu} \sum_{i=1}^{n} z_{i} w_{i}\left(1+\frac{z_{i}^{2}}{\nu}\right)^{-2} r\left(\lambda z_{i} w_{i} \mid \nu+1\right) \\
& +\frac{\lambda^{3}}{\eta^{2}} \frac{\nu+2}{\nu} \sum_{i=1}^{n} z_{i} w_{i}\left(1+\frac{z_{i}^{2}}{\nu}\right)^{-2}\left(1+\left(1+\lambda^{2}\right) \frac{z_{i}^{2}}{\nu}\right)^{-1} r\left(\lambda z_{i} w_{i} \mid \nu+1\right) \\
& +\frac{\lambda^{2}}{\eta^{2}} \frac{\nu+1}{\nu} \sum_{i=1}^{n}\left(1+\frac{z_{i}^{2}}{\nu}\right)^{-3}\left[r\left(\lambda z_{i} w_{i} \mid \nu+1\right)\right]^{2} \\
& -\frac{\lambda}{\eta^{2}} \frac{\alpha-1}{\pi} \sum_{i=1}^{n}\left(1+\frac{z_{i}^{2}}{\nu}\right)^{-1}\left(1+\left(1+\lambda^{2}\right) \frac{z_{i}^{2}}{\nu}\right)^{-1} R_{\lambda}\left(z_{i} \mid \nu\right) \\
& +\frac{\alpha-1}{\eta^{2}} \frac{\nu+1}{\nu} \sum_{i=1}^{n} z_{i}\left(1+\frac{z_{i}^{2}}{\nu}\right)^{-1} r_{\lambda}\left(z_{i} \mid \nu\right)+\frac{\alpha-1}{\eta^{2}} \sum_{i=1}^{n}\left[r_{\lambda}\left(z_{i} \mid \nu\right)\right]^{2} \\
& j_{\xi \eta}=\frac{2}{\eta^{2}} \frac{\nu+1}{\nu} \sum_{i=1}^{n} z_{i}\left(1+\frac{z_{i}^{2}}{\nu}\right)^{-2} \\
& +\frac{\lambda^{3}}{\eta^{2}} \frac{\nu+2}{\nu} \sum_{i=1}^{n} z_{i}^{2} w_{i}\left(1+\frac{z_{i}^{2}}{\nu}\right)^{-2}\left(1+\left(1+\lambda^{2}\right) \frac{z_{i}^{2}}{\nu}\right)^{-1} r\left(\lambda z_{i} w_{i} \mid \nu+1\right) \\
& +\frac{2}{\eta^{2}} \frac{\lambda}{\nu} \sum_{i=1}^{n} z_{i}^{2} w_{i}\left(1+\frac{z_{i}^{2}}{\nu}\right)^{-2} r\left(\lambda z_{i} w_{i} \mid \nu+1\right) \\
& -\frac{\lambda}{\eta^{2}} \sum_{i=1}^{n} w_{i}\left(1+\frac{z_{i}^{2}}{\nu}\right)^{-2} r\left(\lambda z_{i} w_{i} \mid \nu+1\right) \\
& +\frac{\lambda^{2}}{\eta^{2}} \frac{\nu+1}{\nu} \sum_{i=1}^{n} z_{i}\left(1+\frac{z_{i}^{2}}{\nu}\right)^{-3}\left[r\left(\lambda z_{i} w_{i} \mid \nu+1\right)\right]^{2} \\
& -\frac{\lambda}{\eta^{2}} \frac{\alpha-1}{\pi} \sum_{i=1}^{n} z_{i}\left(1+\frac{z_{i}^{2}}{\nu}\right)^{-1}\left(1+\left(1+\lambda^{2}\right) \frac{z_{i}^{2}}{\nu}\right)^{-1} R_{\lambda}\left(z_{i} \mid \nu\right) \\
& -\frac{\alpha-1}{\eta^{2}} \sum_{i=1}^{n} r_{\lambda}\left(z_{i} \mid \nu\right)+\frac{\alpha-1}{\eta^{2}} \frac{\nu+1}{\nu} \sum_{i=1}^{n} z_{i}^{2}\left(1+\frac{z_{i}^{2}}{\nu}\right)^{-1} r_{\lambda}\left(z_{i} \mid \nu\right) \\
& +\frac{\alpha-1}{\eta^{2}} \sum_{i=1}^{n} z_{i}\left[r_{\lambda}\left(z_{i} \mid \nu\right)\right]^{2}
\end{aligned}
$$




$$
\begin{aligned}
& j_{\xi \lambda}=-\frac{\lambda^{2}}{\eta} \frac{\nu+2}{\nu} \sum_{i=1}^{n} z_{i}^{2} w_{i}\left(1+\frac{z_{i}^{2}}{\nu}\right)^{-1}\left(1+\left(1+\lambda^{2}\right) \frac{z_{i}^{2}}{\nu}\right)^{-1} r\left(\lambda z_{i} w_{i} \mid \nu+1\right) \\
& +\frac{1}{\eta} \sum_{i=1}^{n} w_{i}\left(1+\frac{z_{i}^{2}}{\nu}\right)^{-1} r\left(\lambda z_{i} w_{i} \mid \nu+1\right) \\
& -\frac{\lambda}{\eta} \frac{\nu+1}{\nu} \sum_{i=1}^{n} z_{i}\left(1+\frac{z_{i}^{2}}{\nu}\right)^{-2}\left[r\left(\lambda z_{i} w_{i} \mid \nu+1\right)\right]^{2} \\
& +\frac{\alpha-1}{\pi \eta} \sum_{i=1}^{n} z_{i}^{2}\left(1+\frac{z_{i}^{2}}{\nu}\right)^{-1} R_{\lambda}\left(z_{i} \mid \nu\right) \\
& +\frac{\alpha-1}{\pi \eta} \frac{1}{1+\lambda^{2}} \sum_{i=1}^{n} r_{\lambda}\left(z_{i} \mid \nu\right) R_{\lambda}\left(z_{i} \mid \nu\right) \\
& j_{\xi \alpha}=\frac{1}{\eta} \sum_{i=1}^{n} r_{\lambda}\left(z_{i} \mid \nu\right) \\
& j_{\xi \nu}=-\frac{1}{\eta \nu} \sum_{i=1}^{n} z_{i}\left(1+\frac{z_{i}^{2}}{\nu}\right)^{-1}+\frac{\nu+1}{\eta \nu^{2}} \sum_{i=1}^{n} z_{i}\left(1+\frac{z_{i}^{2}}{\nu}\right)^{-2} \\
& +\frac{\lambda}{\eta \nu^{2}} \sum_{i=1}^{n} z_{i}^{2} w_{i}\left(1+\frac{z_{i}^{2}}{\nu}\right)^{-2} r\left(\lambda z_{i} w_{i} \mid \nu+1\right) \\
& +\frac{\lambda}{2 \eta} \frac{1}{\nu(\nu+1)} \sum_{i=1}^{n}\left(z_{i}^{2}-1\right) w_{i}\left(1+\frac{z_{i}^{2}}{\nu}\right)^{-2} r\left(\lambda z_{i} w_{i} \mid \nu+1\right) \\
& +\frac{\lambda^{3}}{2 \eta} \frac{\nu+2}{\nu^{2}(\nu+1)} \sum_{i=1}^{n} z_{i}^{2}\left(z_{i}^{2}-1\right) w_{i}\left(1+\frac{z_{i}^{2}}{\nu}\right)^{-2}\left(1+\left(1+\lambda^{2}\right) \frac{z_{i}^{2}}{\nu}\right)^{-1} r\left(\lambda z_{i} w_{i} \mid \nu+1\right) \\
& -\frac{\lambda^{2}}{2 \eta} \frac{1}{\nu^{2}} \sum_{i=1}^{n} z_{i}\left(z_{i}^{2}-1\right)\left(1+\frac{z_{i}^{2}}{\nu}\right)^{-3}\left[r\left(\lambda z_{i} w_{i} \mid \nu+1\right)\right]^{2} \\
& +\frac{\lambda}{2 \pi \eta} \frac{\alpha-1}{\nu+1} \sum_{i=1}^{n} z_{i}\left(1+\left(1+\lambda^{2}\right) \frac{z_{i}^{2}}{\nu}\right)^{-1} R_{\lambda}\left(z_{i} \mid \nu\right) \\
& +\frac{\alpha-1}{2 \pi \eta(\nu+1)} \frac{\lambda}{1+\lambda^{2}} \sum_{i=1}^{n} r_{\lambda}\left(z_{i} \mid \nu\right) R_{\lambda}\left(z_{i} \mid \nu\right) \\
& +\frac{\alpha-1}{2 \eta} \frac{\nu+1}{\nu^{2}} \sum_{i=1}^{n} z_{i}^{2}\left(1+\frac{z_{i}^{2}}{\nu}\right)^{-1} r_{\lambda}\left(z_{i} \mid \nu\right) \\
& -\frac{\alpha-1}{2 \eta} \sum_{i=1}^{n} \log \left(1+\frac{z_{i}^{2}}{\nu}\right) r_{\lambda}\left(z_{i} \mid \nu\right) \\
& -\frac{\alpha-1}{2 \eta} \sum_{i=1}^{n} \frac{g\left(z_{i}, \nu\right)}{F_{S T}\left(z_{i} \mid \lambda, \nu\right)} r_{\lambda}\left(z_{i} \mid \nu\right) \\
& +\frac{\lambda}{2 \pi \eta} \frac{\alpha-1}{\nu} \sum_{i=1}^{n} z_{i}\left(1+\frac{z_{i}^{2}}{\nu}\right)^{-1}\left(1+\left(1+\lambda^{2}\right) \frac{z_{i}^{2}}{\nu}\right)^{-1} R_{\lambda}\left(z_{i} \mid \nu\right)
\end{aligned}
$$




$$
\begin{aligned}
& j_{\eta \eta}=-\frac{n}{\eta^{2}}+\frac{1}{\eta^{2}} \frac{\nu+1}{\nu} \sum_{i=1}^{n} z_{i}^{2}\left(1+\frac{z_{i}^{2}}{\nu}\right)^{-1}+\frac{2}{\eta^{2}} \frac{\nu+1}{\nu} \sum_{i=1}^{n} z_{i}^{2}\left(1+\frac{z_{i}^{2}}{\nu}\right)^{-2} \\
& -\frac{2 \lambda}{\eta^{2}} \sum_{i=1}^{n} z_{i} w_{i}\left(1+\frac{z_{i}^{2}}{\nu}\right)^{-2} r\left(\lambda z_{i} w_{i} \mid \nu+1\right) \\
& -\frac{\lambda}{\eta^{2} \nu} \sum_{i=1}^{n} z_{i}^{3} w_{i}\left(1+\frac{z_{i}^{2}}{\nu}\right)^{-2} r\left(\lambda z_{i} w_{i} \mid \nu+1\right) \\
& +\frac{\lambda^{3}}{\eta^{2}} \frac{\nu+2}{\nu} \sum_{i=1}^{n} z_{i}^{3} w_{i}\left(1+\frac{z_{i}^{2}}{\nu}\right)^{-2}\left(1+\left(1+\lambda^{2}\right) \frac{z_{i}^{2}}{\nu}\right)^{-1} r\left(\lambda z_{i} w_{i} \mid \nu+1\right) \\
& +\frac{\lambda^{2}}{\eta^{2}} \frac{\nu+1}{\nu} \sum_{i=1}^{n} z_{i}^{2}\left(1+\frac{z_{i}^{2}}{\nu}\right)^{-3} r\left(\lambda z_{i} w_{i} \mid \nu+1\right) \\
& -\frac{\lambda}{\eta^{2}} \frac{\alpha-1}{\pi} \sum_{i=1}^{n} z_{i}^{2}\left(1+\frac{z_{i}^{2}}{\nu}\right)^{-1}\left(1+\left(1+\lambda^{2}\right) \frac{z_{i}^{2}}{\nu}\right)^{-1} R_{\lambda}\left(z_{i} \mid \nu\right) \\
& -\frac{2(\alpha-1)}{\eta^{2}} \sum_{i=1}^{n} z_{i} r_{\lambda}\left(z_{i} \mid \nu\right)-\frac{\alpha-1}{\eta^{2}} \frac{\nu+1}{\nu} \sum_{i=1}^{n} z_{i}^{3}\left(1+\frac{z_{i}^{2}}{\nu}\right)^{-1} r_{\lambda}\left(z_{i} \mid \nu\right) \\
& +\frac{\alpha-1}{\eta^{2}} \sum_{i=1}^{n} z_{i}^{2} r_{\lambda}\left(z_{i} \mid \nu\right) \\
& j_{\eta \lambda}=\frac{1}{\eta} \sum_{i=1}^{n} z_{i} w_{i}\left(1+\frac{z_{i}^{2}}{\nu}\right)^{-1} r\left(\lambda z_{i} w_{i} \mid \nu+1\right) \\
& +\frac{\lambda^{2}}{\eta} \frac{\nu+2}{\nu} \sum_{i=1}^{n} z_{i}^{3} w_{i}\left(1+\frac{z_{i}^{2}}{\nu}\right)^{-1}\left(1+\left(1+\lambda^{2}\right) \frac{z_{i}^{2}}{\nu}\right)^{-1} r\left(\lambda z_{i} w_{i} \mid \nu+1\right) \\
& +\frac{\lambda}{\eta} \frac{\nu+1}{\nu} \sum_{i=1}^{n} z_{i}\left(1+\frac{z_{i}^{2}}{\nu}\right)^{-2}\left[r\left(\lambda z_{i} w_{i} \mid \nu+1\right)\right]^{2} \\
& -\frac{\alpha-1}{\pi \eta} \sum_{i=1}^{n} z_{i}^{2}\left(1+\frac{z_{i}^{2}}{\nu}\right)^{-1} R_{\lambda}\left(z_{i} \mid \nu\right) \\
& +\frac{\alpha-1}{\pi \eta} \frac{1}{1+\lambda^{2}} \sum_{i=1}^{n} r_{\lambda}\left(z_{i} \mid \nu\right) R_{\lambda}\left(z_{i} \mid \nu\right) \\
& j_{\eta \lambda}=\frac{1}{\eta} \sum_{i=1}^{n} z_{i} r_{\lambda}\left(z_{i} \mid \nu\right)
\end{aligned}
$$




$$
\begin{aligned}
& j_{\eta \nu}=-\frac{1}{\eta \nu} \sum_{i=1}^{n} z_{i}^{2}\left(1+\frac{z_{i}^{2}}{\nu}\right)^{-1}+\frac{1}{\eta} \frac{\nu+1}{\nu^{2}} \sum_{i=1}^{n} z_{i}^{2}\left(1+\frac{z_{i}^{2}}{\nu}\right)^{-2} \\
& +\frac{\lambda}{\eta \nu^{2}} \sum_{i=1}^{n} z_{i}^{3} w_{i}\left(1+\frac{z_{i}^{2}}{\nu}\right)^{-2} r\left(\lambda z_{i} w_{i} \mid \nu+1\right) \\
& +\frac{\lambda}{2 \eta} \frac{1}{\nu(\nu+1)} \sum_{i=1}^{n} z_{i}\left(z_{i}^{2}-1\right) w_{i}\left(1+\frac{z_{i}^{2}}{\nu}\right)^{-2} r\left(\lambda z_{i} w_{i} \mid \nu+1\right) \\
& -\frac{\lambda^{3}}{2 \eta} \frac{\nu+2}{\nu^{2}(\nu+1)} \sum_{i=1}^{n} z_{i}^{3}\left(z_{i}^{2}-1\right) w_{i}\left(1+\frac{z_{i}^{2}}{\nu}\right)^{-2}\left(1+\left(1+\lambda^{2}\right) \frac{z_{i}^{2}}{\nu}\right)^{-1} r\left(\lambda z_{i} w_{i} \mid \nu+1\right) \\
& -\frac{\lambda^{2}}{2 \eta \nu^{2}} \sum_{i=1}^{n} z_{i}^{2}\left(z_{i}^{2}-1\right)\left(1+\frac{z_{i}^{2}}{\nu}\right)^{-3}\left[r\left(\lambda z_{i} w_{i} \mid \nu+1\right)\right]^{2} \\
& +\frac{\lambda}{2 \pi \eta} \frac{\alpha-1}{\nu(\nu+1)} \sum_{i=1}^{n} z_{i}^{2}\left(z_{i}^{2}-1\right)\left(1+\frac{z_{i}^{2}}{\nu}\right)^{-1}\left(1+\left(1+\lambda^{2}\right) \frac{z_{i}^{2}}{\nu}\right)^{-1} R_{\lambda}\left(z_{i} \mid \nu\right) \\
& +\frac{\alpha-1}{2 \pi \eta(\nu+1)} \frac{\lambda}{1+\lambda^{2}} \sum_{i=1}^{n} z_{i} r_{\lambda}\left(z_{i} \mid \nu\right) R_{\lambda}\left(z_{i} \mid \nu\right) \\
& +\frac{\alpha-1}{2 \eta} \frac{\nu+1}{\nu^{2}} \sum_{i=1}^{n} z_{i}^{3}\left(1+\frac{z_{i}^{2}}{\nu}\right)^{-1} r\left(\lambda z_{i} w_{i} \mid \nu+1\right) \\
& -\frac{\alpha-1}{2 \eta} \sum_{i=1}^{n} z_{i} \log \left(1+\frac{z_{i}^{2}}{\nu}\right) r\left(\lambda z_{i} w_{i} \mid \nu+1\right) \\
& -\frac{\alpha-1}{2 \eta} \sum_{i=1}^{n} z_{i} \frac{g\left(z_{i}, \nu\right)}{F_{S T}\left(z_{i} \mid \lambda, \nu\right)} r_{\lambda}\left(z_{i} \mid \nu\right) \\
& j_{\lambda \lambda}=\frac{\lambda(\nu+2)}{\nu} \sum_{i=1}^{n} z_{i}^{3} w_{i}\left(1+\left(1+\lambda^{2}\right) \frac{z_{i}^{2}}{\nu}\right)^{-1} r\left(\lambda z_{i} w_{i} \mid \nu+1\right) \\
& +\frac{\nu+1}{\nu} \sum_{i=1}^{n} z_{i}\left(1+\frac{z_{i}^{2}}{\nu}\right)^{-1}\left[r\left(\lambda z_{i} w_{i} \mid \nu+1\right)\right]^{2} \\
& -\frac{2(\alpha-1)}{\pi} \frac{\lambda}{\left(1+\lambda^{2}\right)^{2}} \sum_{i=1}^{n}\left(1+\left(1+\lambda^{2}\right) \frac{z_{i}^{2}}{\nu}\right)^{-1} R_{\lambda}\left(z_{i} \mid \nu\right) \\
& -\frac{\alpha-1}{\pi} \frac{\lambda}{1+\lambda^{2}} \frac{\nu+2}{\nu} \sum_{i=1}^{n} z_{i}^{2}\left(1+\left(1+\lambda^{2}\right) \frac{z_{i}^{2}}{\nu}\right)^{-1} R_{\lambda}\left(z_{i} \mid \nu\right) \\
& +\frac{\alpha-1}{\pi^{2}\left(1+\lambda^{2}\right)^{2}} \sum_{i=1}^{n}\left[R_{\lambda}\left(z_{i} \mid \nu\right)\right]^{2} \\
& j_{\lambda \alpha}=\frac{1}{\pi\left(1+\lambda^{2}\right)} \sum_{i=1}^{n} R_{\lambda}\left(z_{i} \mid \nu\right)
\end{aligned}
$$




$$
\begin{aligned}
j_{\lambda \nu}= & -\frac{1}{2 \nu(\nu+1)} \sum_{i=1}^{n} z_{i}\left(z_{i}^{2}-1\right) w_{i}\left(1+\frac{z_{i}^{2}}{\nu}\right)^{-1} r\left(\lambda z_{i} w_{i} \mid \nu+1\right) \\
& +\frac{\lambda^{2}}{2 \nu^{2}} \frac{\nu+2}{(\nu+1)^{2}} \sum_{i=1}^{n} z_{i}^{3}\left(z_{i}^{2}-1\right) w_{i}\left(1+\frac{z_{i}^{2}}{\nu}\right)^{-1}\left(1+\left(1+\lambda^{2}\right) \frac{z_{i}^{2}}{\nu}\right)^{-1} r\left(\lambda z_{i} w_{i} \mid \nu+1\right) \\
& +\frac{\lambda}{2 \nu^{2}} \sum_{i=1}^{n} z_{i}^{2}\left(z_{i}^{2}-1\right)\left(1+\frac{z_{i}^{2}}{\nu}\right)^{-2}\left[r\left(\lambda z_{i} w_{i} \mid \nu+1\right)\right]^{2} \\
& +\frac{\alpha-1}{2 \pi(\nu+1)} \frac{1-\lambda^{2}}{\left(1+\lambda^{2}\right)^{2}} \sum_{i=1}^{n} R_{\lambda}\left(z_{i} \mid \nu\right)+\frac{\alpha-1}{2 \pi(\nu+1)} \frac{\lambda}{\left(1+\lambda^{2}\right)^{2}} \sum_{i=1}^{n}\left[R_{\lambda}\left(z_{i} \mid \nu\right)\right]^{2} \\
& -\frac{\alpha-1}{2 \pi(\nu+1)} \frac{\lambda^{2}}{1+\lambda^{2}} \sum_{i=1}^{n} z_{i}^{2}\left(1+\left(1+\lambda^{2}\right) \frac{z_{i}^{2}}{\nu}\right)^{-1} R_{\lambda}\left(z_{i} \mid \nu\right) \\
& -\frac{\alpha-1}{2 \pi\left(1+\lambda^{2}\right)} \sum_{i=1}^{n} \frac{g\left(z_{i}, \nu\right)}{F_{S T}\left(z_{i} \mid \lambda, \nu\right)} R_{\lambda}\left(z_{i} \mid \nu\right) \\
& -\frac{\alpha-1}{2 \pi} \sum_{i=1}^{n} \frac{g_{1}\left(z_{i}, \nu\right)}{F_{S T}\left(z_{i} \mid \lambda, \nu\right)} \\
j_{\alpha \alpha}= & \frac{n}{\alpha^{2}} \\
& +\frac{n}{2 \pi(\nu+1)} \frac{\lambda}{1+\lambda^{2}} \sum_{i=1}^{n} R_{\lambda}\left(z_{i} \mid \nu\right)-\frac{1}{2} \sum_{i=1} \frac{g\left(z_{i}, \nu\right)}{F_{S T}\left(x_{i} \mid \lambda, \nu\right)} \\
j_{\alpha \nu}\left(\frac{\nu+1}{2}\right)+\frac{n}{2} \psi\left(\frac{\nu}{2}\right)+\frac{n}{2 \nu} & \left.-\frac{1}{2}{ }^{2}\right) \\
& \\
&
\end{aligned}
$$




$$
\begin{aligned}
& j_{\nu \nu}=-\frac{n \alpha}{2 \nu^{2}}-\frac{n \alpha}{4} \psi_{1}\left(\frac{\nu+1}{2}\right)+\frac{n \alpha}{4} \psi_{1}\left(\frac{\nu}{2}\right)-\frac{\nu-1}{2 \nu^{3}} \sum_{i=1}^{n} z_{i}^{2}\left(1+\frac{z_{i}^{2}}{\nu}\right)^{-1} \\
& +\frac{\nu+1}{2 \nu^{3}} \sum_{i=1}^{n} z_{i}^{2}\left(1+\frac{z_{i}^{2}}{\nu}\right)^{-2} \\
& +\frac{\lambda}{4(\nu+1)^{2}} \frac{1}{\nu^{2}} \sum_{i=1}^{n} z_{i}\left(z_{i}^{2}-1\right)\left(z_{i}^{2}+4 \nu+3\right) w_{i}\left(1+\frac{z_{i}^{2}}{\nu}\right)^{-2} r\left(\lambda z_{i} w_{i} \mid \nu+1\right) \\
& -\frac{\lambda}{4 \nu(\nu+1)}\left(\psi\left(\frac{\nu+2}{2}\right)-\psi\left(\frac{\nu+1}{2}\right)-\frac{1}{\nu+1}\right) \\
& \sum_{i=1}^{n} z_{i}\left(z_{i}^{2}-1\right) w_{i}\left(1+\frac{z_{i}^{2}}{\nu}\right)^{-1} r\left(\lambda z_{i} w_{i} \mid \nu+1\right) \\
& -\frac{\lambda^{3}}{4(\nu+1)} \frac{\nu+2}{\nu^{3}} \sum_{i=1}^{n} z_{i}^{3}\left(z_{i}^{2}-1\right)\left(1+\frac{z_{i}^{2}}{\nu}\right)^{-2}\left(1+\left(1+\lambda^{2}\right) \frac{z_{i}^{2}}{\nu}\right)^{-1} r\left(\lambda z_{i} w_{i} \mid \nu+1\right) \\
& +\frac{\lambda}{4 \nu(\nu+1)} \sum_{i=1}^{n} z_{i}\left(z_{i}^{2}-1\right) \log \left(1+\frac{\lambda^{2} z_{i}^{2}}{\nu+z_{i}^{2}}\right) r\left(\lambda z_{i} w_{i} \mid \nu+1\right) \\
& +\frac{\lambda^{2}}{4 \nu^{3}(\nu+1)} \sum_{i=1}^{n} z_{i}^{2}\left(z_{i}^{2}-1\right)^{2}\left(1+\frac{z_{i}^{2}}{\nu}\right)^{-3}\left[r\left(\lambda z_{i} w_{i} \mid \nu+1\right)\right]^{2} \\
& -\frac{\alpha-1}{2 \pi(\nu+1)^{2}} \frac{\lambda}{1+\lambda^{2}} \sum_{i=1}^{n} R_{\lambda}\left(z_{i} \mid \nu\right) \\
& +\frac{\alpha-1}{4 \pi(\nu+1)} \frac{\lambda}{\nu} \sum_{i=1}^{n} z_{i}^{2}\left(1+\left(1+\lambda^{2}\right) \frac{z_{i}^{2}}{\nu}\right)^{-1} R_{\lambda}\left(z_{i} \mid \nu\right) \\
& -\frac{\alpha-1}{4 \pi(\nu+1)} \frac{\lambda}{1+\lambda^{2}} \sum_{i=1}^{n} \log \left(1+\left(1+\lambda^{2}\right) \frac{z_{i}^{2}}{\nu}\right) R_{\lambda}\left(z_{i} \mid \nu\right) \\
& +\frac{\alpha-1}{4 \pi(\nu+1)} \frac{\lambda}{1+\lambda^{2}}\left(\psi\left(\frac{\nu+1}{2}\right)-\psi\left(\frac{\nu}{2}\right)-\frac{1}{\nu}\right) \sum_{i=1}^{n} R_{\lambda}\left(z_{i} \mid \nu\right) \\
& -\frac{\alpha-1}{2 \pi(\nu+1)} \frac{\lambda}{1+\lambda^{2}} \sum_{i=1}^{n} \frac{g\left(z_{i}, \nu\right)}{F_{S T}\left(z_{i} \mid \lambda, \nu\right)} R_{\lambda}\left(z_{i} \mid \nu\right) \\
& +\frac{\alpha-1}{4 \pi^{2}(\nu+1)^{2}} \frac{\lambda^{2}}{\left(1+\lambda^{2}\right)^{2}} \sum_{i=1}^{n}\left[R_{\lambda}\left(z_{i} \mid \nu\right)\right]^{2} \\
& +\frac{\alpha-1}{4} \sum_{i=1}^{n}\left(\frac{g\left(z_{i}, \nu\right)}{F_{S T}\left(z_{i} \mid \lambda, \nu\right)}\right)^{2}-\frac{\alpha-1}{2} \sum_{i=1}^{n} \frac{g_{2}\left(z_{i}, \nu\right)}{F_{S T}\left(z_{i} \mid \lambda, \nu\right)}
\end{aligned}
$$

em que $w=\sqrt{\frac{\nu+1}{z^{2}+\nu}}, r(z \mid \nu)=\frac{t(z \mid \nu)}{T(z \mid \nu)}, R_{\lambda}(z \mid \nu)=\frac{1}{F_{S T}(z \mid \lambda, \nu)}\left\{1+\left(1+\lambda^{2}\right) z^{2} / \nu\right\}^{-\frac{\nu}{2}}$, e $r_{\lambda}(z \mid \nu)=\frac{f_{S T}(z \mid \lambda, \nu)}{F_{S T}(z \mid \lambda, \nu)}$; e $g(z ; \nu), g_{1}(z ; \nu)$ e $g_{2}(z ; \nu)$ são dados em (3.9), (B.1) e (B.2) respectivamente. 


\section{B.2 Matriz de Informação Observada: Modelo PT Censurado}

As expressões para os elementos da matriz de informação observada do modelo PT censurado são dados a seguir. Estes elementos são obtidos calculando a segunda derivada da função de logverossimilhança $\ell\left(\boldsymbol{\varphi} ; \mathbf{Y}^{o}\right)$ em (3.24), em que $\boldsymbol{\varphi}=(\gamma, \sigma, \alpha, \nu)^{\top}$ e multiplicando por -1 .

$$
\begin{aligned}
j_{\gamma \gamma}=\alpha & \sum_{i=1}^{n}\left(1-d_{i}\right)\left\{r\left(c_{i} \mid \nu\right)+\left(\frac{\nu+1}{\nu}\right)\left(1+\frac{c_{i}^{2}}{\nu}\right)^{-1} c_{i}\right\} r\left(c_{i} \mid \nu\right) \\
& +\left(\frac{\nu+1}{\nu}\right) \sum_{i=1}^{n} d_{i}\left\{\left(1+\frac{z_{i}^{2}}{\nu}\right)^{-1}-\frac{2}{\nu}\left(1+\frac{z_{i}^{2}}{\nu}\right)^{-2} z_{i}^{2}\right\} \\
& +(\alpha-1) \sum_{i=1}^{n} d_{i}\left\{r\left(z_{i} \mid \nu\right)+\left(\frac{\nu+1}{\nu}\right)\left(1+\frac{z_{i}^{2}}{\nu}\right)^{-1} z_{i}\right\} r\left(z_{i} \mid \nu\right) \\
j_{\gamma \sigma}=- & \alpha \sum_{i=1}^{n}\left(1-d_{i}\right)\left\{r\left(c_{i} \mid \nu\right)+\left(\frac{\nu+1}{\nu}\right)\left(1+\frac{c_{i}^{2}}{\nu}\right)^{-1} c_{i}\right\} k_{i} r\left(c_{i} \mid \nu\right) \\
& -\left(\frac{\nu+1}{\nu}\right) \sum_{i=1}^{n} d_{i}\left\{\left(1+\frac{z_{i}^{2}}{\nu}\right)^{-1}-\frac{2}{\nu}\left(1+\frac{z_{i}^{2}}{\nu}\right)^{-2} z_{i}^{2}\right\} y_{i} \\
& -(\alpha-1) \sum_{i=1}^{n} d_{i}\left\{r\left(z_{i} \mid \nu\right)+\left(\frac{\nu+1}{\nu}\right)\left(1+\frac{z_{i}^{2}}{\nu}\right)^{-1} z_{i}\right\} y_{i} r\left(z_{i} \mid \nu\right)
\end{aligned}
$$




$$
\begin{aligned}
& j_{\gamma \nu}=\frac{\alpha}{2} \sum_{i=1}^{n} d_{i}\left\{\left(\frac{\nu+1}{\nu}\right)\left(1+\frac{c_{i}^{2}}{\nu}\right)^{-1} \frac{c_{i}^{2}}{\nu}+b_{01}\left(c_{i} \mid \nu\right)\right. \\
& \left.-\log \left(1+\frac{c_{i}^{2}}{\nu}\right)-\frac{1}{\nu}\left(1-c_{i} r\left(c_{i} \mid \nu\right)\right)\right\} r\left(c_{i} \mid \nu\right) \\
& +\frac{1}{\nu^{2}} \sum_{i=1}^{n} d_{i}\left\{\left(1+\frac{z_{i}^{2}}{\nu}\right)^{-1} z_{i}-\left(\frac{\nu+1}{\nu}\right)\left(1+\frac{z_{i}^{2}}{\nu}\right)^{-2} z_{i}^{3}\right\} \\
& +\frac{\alpha-1}{2} \sum_{i=1}^{n} d_{i}\left\{\left(\frac{\nu+1}{\nu}\right)\left(1+\frac{z_{i}^{2}}{\nu}\right)^{-1} \frac{z_{i}^{2}}{\nu}+b_{01}\left(z_{i} \mid \nu\right)\right. \\
& \left.-\log \left(1+\frac{z_{i}^{2}}{\nu}\right)-\frac{1}{\nu}\left(1-z_{i} r\left(z_{i} \mid \nu\right)\right)\right\} r\left(z_{i} \mid \nu\right) \\
& j_{\gamma \alpha}=\sum_{i=1}^{n}\left(1-d_{i}\right) r\left(c_{i} \mid \nu\right)+\sum_{i=1}^{n} d_{i} r\left(z_{i} \mid \nu\right) \\
& j_{\sigma \sigma}=\alpha \sum_{i=1}^{n}\left(1-d_{i}\right)\left\{\left(\frac{\nu+1}{\nu}\right)\left(1+\frac{c_{i}^{2}}{\nu}\right)^{-1} c_{i}+r\left(c_{i} \mid \nu\right)\right\} r\left(c_{i} \mid \nu\right) k_{i}^{2} \\
& +\sum_{i=1}^{n} d_{i}\left\{\frac{1}{\sigma^{2}}+\left(\frac{\nu+1}{\nu}\right)\left[\left(1+\frac{z_{i}^{2}}{\nu}\right)^{-1}-\frac{2}{\nu}\left(1+\frac{z_{i}^{2}}{\nu}\right)^{-2} z_{i}^{2}\right] y_{i}^{2}\right\} \\
& +(\alpha-1) \sum_{i=1}^{n} d_{i}\left\{\left(\frac{\nu+1}{\nu}\right)\left(1+\frac{z_{i}^{2}}{\nu}\right)^{-1} z_{i}+r\left(z_{i} \mid \nu\right)\right\} r\left(z_{i} \mid \nu\right) y_{i}^{2} \\
& j_{\sigma \nu}=-\frac{\alpha}{2} \sum_{i=1}^{n}\left(1-d_{i}\right)\left\{\left(\frac{\nu+1}{\nu}\right)\left(1+\frac{c_{i}^{2}}{\nu}\right)^{-1} \frac{c_{i}^{2}}{\nu}+b_{01}\left(c_{i} \mid \nu\right)\right. \\
& \left.-\log \left(1+\frac{c_{i}^{2}}{\nu}\right)-\frac{1}{\nu}\left(1-c_{i} r\left(c_{i} \mid \nu\right)\right)\right\} k_{i} r\left(c_{i} \mid \nu\right) \\
& -\frac{1}{\nu^{2}} \sum_{i=1}^{n} d_{i}\left\{\left(1+\frac{z_{i}^{2}}{\nu}\right)^{-1} z_{i}-\left(\frac{\nu+1}{\nu}\right)\left(1+\frac{z_{i}^{2}}{\nu}\right)^{-2} z_{i}^{3}\right\} y_{i} \\
& -\frac{\alpha-1}{2} \sum_{i=1}^{n} d_{i}\left\{\left(\frac{\nu+1}{\nu}\right)\left(1+\frac{z_{i}^{2}}{\nu}\right)^{-1} \frac{z_{i}^{2}}{\nu}+b_{01}\left(z_{i} \mid \nu\right)\right. \\
& \left.-\log \left(1+\frac{z_{i}^{2}}{\nu}\right)-\frac{1}{\nu}\left(1-z_{i} r\left(z_{i} \mid \nu\right)\right)\right\} y_{i} r\left(z_{i} \mid \nu\right) \\
& j_{\sigma \alpha}=-\sum_{i=1}^{n}\left(1-d_{i}\right) r\left(c_{i} \mid \nu\right) k_{i}-\sum_{i=1}^{n} d_{i} r\left(z_{i} \mid \nu\right) y_{i}
\end{aligned}
$$




$$
\begin{aligned}
& j_{\nu \nu}=\frac{\alpha}{4} \sum_{i=1}^{n}\left(1-d_{i}\right)\left\{\psi_{1}\left(\frac{\nu}{2}\right)-\psi_{1}\left(\frac{\nu+1}{2}\right)-\frac{2}{\nu(\nu+1)}+b_{01}^{2}\left(c_{i} \mid \nu\right)\right. \\
& -\frac{1}{\nu} b_{01}\left(c_{i} \mid \nu\right)+\frac{1}{\nu}\left(2 b_{01}\left(c_{i} \mid \nu\right)-\frac{\nu+3}{\nu(\nu+1)}\right) c_{i} r\left(c_{i} \mid \nu\right) \\
& +\frac{1}{\nu+2} \frac{T\left(c_{2 i} \mid \nu+2\right)}{T\left(c_{i} \mid \nu\right)} b_{21}\left(c_{2 i} \mid \nu+2\right)-b_{02}\left(c_{i} \mid \nu\right) \\
& \left.+\frac{1}{\nu}\left[\left(\frac{\nu+1}{\nu}\right)\left(1+\frac{c_{i}^{2}}{\nu}\right)^{-1} \frac{c_{i}^{2}}{\nu}-\log \left(1+\frac{c_{i}^{2}}{\nu}\right)+\frac{c_{i}}{\nu} r\left(c_{i} \mid \nu\right)\right] c_{i} r\left(c_{i} \mid \nu\right)\right\} \\
& -\frac{1}{4} \sum_{i=1}^{n} d_{i}\left\{\psi_{1}\left(\frac{\nu+1}{2}\right)-\psi_{1}\left(\frac{\nu}{2}\right)+\frac{2}{\nu^{2}}-\frac{4}{\nu^{3}}\left(1+\frac{z_{i}^{2}}{\nu}\right)^{-1} z_{i}^{2}\right. \\
& \left.+\frac{2}{\nu^{3}}\left(\frac{\nu+1}{\nu}\right)\left(1+\frac{z_{i}^{2}}{\nu}\right)^{-2} z_{i}^{4}\right\} \\
& +\frac{\alpha-1}{4} \sum_{i=1}^{n} d_{i}\left\{\psi_{1}\left(\frac{\nu}{2}\right)-\psi_{1}\left(\frac{\nu+1}{2}\right)-\frac{2}{\nu(\nu+1)}+b_{01}^{2}\left(z_{i} \mid \nu\right)\right. \\
& -\frac{1}{\nu} b_{01}\left(z_{i} \mid \nu\right)+\frac{1}{\nu}\left(2 b_{01}\left(z_{i} \mid \nu\right)-\frac{\nu+3}{\nu(\nu+1)}\right) z_{i} r\left(z_{i} \mid \nu\right) \\
& +\frac{1}{\nu+2} \frac{T\left(z_{2 i} \mid \nu+2\right)}{T\left(z_{i} \mid \nu\right)} b_{21}\left(z_{2 i} \mid \nu+2\right)-b_{02}\left(z_{i} \mid \nu\right) \\
& \left.+\frac{1}{\nu}\left[\left(\frac{\nu+1}{\nu}\right)\left(1+\frac{z_{i}^{2}}{\nu}\right)^{-1} \frac{z_{i}^{2}}{\nu}-\log \left(1+\frac{z_{i}^{2}}{\nu}\right)+\frac{z_{i}}{\nu} r\left(z_{i} \mid \nu\right)\right] z_{i} r\left(z_{i} \mid \nu\right)\right\} \\
& j_{\nu \alpha}=\frac{1}{2} \sum_{i=1}^{n}\left(1-d_{i}\right)\left\{\psi\left(\frac{\nu+1}{2}\right)-\psi\left(\frac{\nu}{2}\right)-b_{01}\left(c_{i} \mid \nu\right)-\frac{c_{i} r\left(c_{i} \mid \nu\right)}{\nu}\right\} \\
& -\frac{1}{2} \sum_{i=1}^{n} d_{i}\left\{\psi\left(\frac{\nu+1}{2}\right)-\psi\left(\frac{\nu}{2}\right)-b_{01}\left(z_{i} \mid \nu\right)-\frac{z_{i} r\left(z_{i} \mid \nu\right)}{\nu}\right\} \\
& j_{\alpha \alpha}=\sum_{i=1}^{n} \frac{d_{i}}{\alpha^{2}}
\end{aligned}
$$

\section{B.3 Matriz de Informação Observada: Modelo PT Truncado}

As expressões para os elementos da matriz de informação observada do modelo PT truncado são dados a seguir. Estes elementos são obtidos calculando a segunda derivada da função de 
MATRIZ DE INFORMAÇÃO OBSERVADA: MODELO PT TRUNCADO

101

log-verossimilhança $\ell(\boldsymbol{\varphi} ; \mathbf{Y})$ em (3.32), em que $\boldsymbol{\varphi}=(\gamma, \sigma, \alpha, \nu)^{\top}$.

$$
\begin{aligned}
& j_{\gamma \gamma}=-n_{1} \alpha\left\{r(c \mid \nu)+\left(\frac{\nu+1}{\nu}\right)\left(1+\frac{c^{2}}{\nu}\right)^{-1} c\right\} r(c \mid \nu) \\
& +\left(\frac{\nu+1}{\nu}\right) \sum_{y_{i} \leq k}\left\{\left(1+\frac{z_{i}^{2}}{\nu}\right)^{-1}-\frac{2}{\nu}\left(1+\frac{z_{i}^{2}}{\nu}\right)^{-2} z_{i}^{2}\right\} \\
& +(\alpha-1) \sum_{y_{i} \leq k}\left\{r\left(z_{i} \mid \nu\right)+\left(\frac{\nu+1}{\nu}\right)\left(1+\frac{z_{i}^{2}}{\nu}\right)^{-1} z_{i}\right\} r\left(z_{i} \mid \nu\right) \\
& j_{\gamma \sigma}=n_{1} \alpha\left\{r(c \mid \nu)+\left(\frac{\nu+1}{\nu}\right)\left(1+\frac{c^{2}}{\nu}\right)^{-1} c\right\} k r(c \mid \nu) \\
& -\left(\frac{\nu+1}{\nu}\right) \sum_{y_{i} \leq k}\left\{\left(1+\frac{z_{i}^{2}}{\nu}\right)^{-1}-\frac{2}{\nu}\left(1+\frac{z_{i}^{2}}{\nu}\right)^{-2} z_{i}^{2}\right\} y_{i} \\
& -(\alpha-1) \sum_{y_{i} \leq k}\left\{r\left(z_{i} \mid \nu\right)+\left(\frac{\nu+1}{\nu}\right)\left(1+\frac{z_{i}^{2}}{\nu}\right)^{-1} z_{i}\right\} y_{i} r\left(z_{i} \mid \nu\right) \\
& j_{\gamma \nu}=-\frac{n_{1} \alpha}{2}\left\{\left(\frac{\nu+1}{\nu}\right)\left(1+\frac{c^{2}}{\nu}\right)^{-1} \frac{c^{2}}{\nu}+b_{01}(c \mid \nu)\right. \\
& \left.-\log \left(1+\frac{c_{i}^{2}}{\nu}\right)-\frac{1}{\nu}(1-c r(c \mid \nu))\right\} r(c \mid \nu) \\
& +\frac{1}{\nu^{2}} \sum_{y_{i} \leq k}\left\{\left(1+\frac{z_{i}^{2}}{\nu}\right)^{-1} z_{i}-\left(\frac{\nu+1}{\nu}\right)\left(1+\frac{z_{i}^{2}}{\nu}\right)^{-2} z_{i}^{3}\right\} \\
& +\frac{\alpha-1}{2} \sum_{y_{i} \leq k}\left\{\left(\frac{\nu+1}{\nu}\right)\left(1+\frac{z_{i}^{2}}{\nu}\right)^{-1} \frac{z_{i}^{2}}{\nu}+b_{01}\left(z_{i} \mid \nu\right)\right. \\
& \left.-\log \left(1+\frac{z_{i}^{2}}{\nu}\right)-\frac{1}{\nu}\left(1-z_{i} r\left(z_{i} \mid \nu\right)\right)\right\} r\left(z_{i} \mid \nu\right) \\
& j_{\gamma \alpha}=-n_{1} r(c \mid \nu)+\sum_{y_{i} \leq k} r\left(z_{i} \mid \nu\right) \\
& j_{\sigma \sigma}=-n_{1} \alpha\left\{\left(\frac{\nu+1}{\nu}\right)\left(1+\frac{c^{2}}{\nu}\right)^{-1} c+r(c \mid \nu)\right\} r(c \mid \nu) k^{2} \\
& +\sum_{y_{i} \leq k}\left\{\frac{1}{\sigma^{2}}+\left(\frac{\nu+1}{\nu}\right)\left[\left(1+\frac{z_{i}^{2}}{\nu}\right)^{-1}-\frac{2}{\nu}\left(1+\frac{z_{i}^{2}}{\nu}\right)^{-2} z_{i}^{2}\right] y_{i}^{2}\right\} \\
& +(\alpha-1) \sum_{y_{i} \leq k}\left\{\left(\frac{\nu+1}{\nu}\right)\left(1+\frac{z_{i}^{2}}{\nu}\right)^{-1} z_{i}+r\left(z_{i} \mid \nu\right)\right\} r\left(z_{i} \mid \nu\right) y_{i}^{2}
\end{aligned}
$$




$$
\begin{aligned}
& j_{\sigma \nu}=\frac{n_{1} \alpha}{2}\left\{\left(\frac{\nu+1}{\nu}\right)\left(1+\frac{c^{2}}{\nu}\right)^{-1} \frac{c^{2}}{\nu}+b_{01}(c \mid \nu)\right. \\
& \left.-\log \left(1+\frac{c^{2}}{\nu}\right)-\frac{1}{\nu}(1-c r(c \mid \nu))\right\} k r(c \mid \nu) \\
& -\frac{1}{\nu^{2}} \sum_{y_{i} \leq k}\left\{\left(1+\frac{z_{i}^{2}}{\nu}\right)^{-1} z_{i}-\left(\frac{\nu+1}{\nu}\right)\left(1+\frac{z_{i}^{2}}{\nu}\right)^{-2} z_{i}^{3}\right\} y_{i} \\
& -\frac{\alpha-1}{2} \sum_{y_{i} \leq k}\left\{\left(\frac{\nu+1}{\nu}\right)\left(1+\frac{z_{i}^{2}}{\nu}\right)^{-1} \frac{z_{i}^{2}}{\nu}+b_{01}\left(z_{i} \mid \nu\right)\right. \\
& \left.-\log \left(1+\frac{z_{i}^{2}}{\nu}\right)-\frac{1}{\nu}\left(1-z_{i} r\left(z_{i} \mid \nu\right)\right)\right\} y_{i} r\left(z_{i} \mid \nu\right) \\
& j_{\sigma \alpha}=n_{1} r\left(c_{i} \mid \nu\right) k_{i}-\sum_{y_{i} \leq k} r\left(z_{i} \mid \nu\right) y_{i} \\
& j_{\nu \nu}=-\frac{n_{1} \alpha}{4}\left\{\psi_{1}\left(\frac{\nu}{2}\right)-\psi_{1}\left(\frac{\nu+1}{2}\right)-\frac{2}{\nu(\nu+1)}+b_{01}^{2}(c \mid \nu)\right. \\
& -\frac{1}{\nu} b_{01}(c \mid \nu)+\frac{1}{\nu}\left(2 b_{01}(c \mid \nu)-\frac{\nu+3}{\nu(\nu+1)}\right) \operatorname{cr}(c \mid \nu) \\
& +\frac{1}{\nu+2} \frac{T\left(c_{2} \mid \nu+2\right)}{T(c \mid \nu)} b_{21}\left(c_{2} \mid \nu+2\right)-b_{02}\left(c_{\mid} \nu\right) \\
& \left.+\frac{1}{\nu}\left[\left(\frac{\nu+1}{\nu}\right)\left(1+\frac{c^{2}}{\nu}\right)^{-1} \frac{c^{2}}{\nu}-\log \left(1+\frac{c^{2}}{\nu}\right)+\frac{c}{\nu} r(c \mid \nu)\right] \operatorname{cr}(c \mid \nu)\right\} \\
& -\frac{1}{4} \sum_{y_{i} \leq k}\left\{\psi_{1}\left(\frac{\nu+1}{2}\right)-\psi_{1}\left(\frac{\nu}{2}\right)+\frac{2}{\nu^{2}}-\frac{4}{\nu^{3}}\left(1+\frac{z_{i}^{2}}{\nu}\right)^{-1} z_{i}^{2}\right. \\
& \left.+\frac{2}{\nu^{3}}\left(\frac{\nu+1}{\nu}\right)\left(1+\frac{z_{i}^{2}}{\nu}\right)^{-2} z_{i}^{4}\right\} \\
& +\frac{\alpha-1}{4} \sum_{y_{i} \leq k}\left\{\psi_{1}\left(\frac{\nu}{2}\right)-\psi_{1}\left(\frac{\nu+1}{2}\right)-\frac{2}{\nu(\nu+1)}+b_{01}^{2}\left(z_{i} \mid \nu\right)\right. \\
& -\frac{1}{\nu} b_{01}\left(z_{i} \mid \nu\right)+\frac{1}{\nu}\left(2 b_{01}\left(z_{i} \mid \nu\right)-\frac{\nu+3}{\nu(\nu+1)}\right) z_{i} r\left(z_{i} \mid \nu\right) \\
& +\frac{1}{\nu+2} \frac{T\left(z_{2 i} \mid \nu+2\right)}{T\left(z_{i} \mid \nu\right)} b_{21}\left(z_{2 i} \mid \nu+2\right)-b_{02}\left(z_{i} \mid \nu\right) \\
& \left.+\frac{1}{\nu}\left[\left(\frac{\nu+1}{\nu}\right)\left(1+\frac{z_{i}^{2}}{\nu}\right)^{-1} \frac{z_{i}^{2}}{\nu}-\log \left(1+\frac{z_{i}^{2}}{\nu}\right)+\frac{z_{i}}{\nu} r\left(z_{i} \mid \nu\right)\right] z_{i} r\left(z_{i} \mid \nu\right)\right\} \\
& j_{\nu \alpha}=\frac{n_{1}}{2}\left\{\psi\left(\frac{\nu+1}{2}\right)-\psi\left(\frac{\nu}{2}\right)-b_{01}\left(c_{i} \mid \nu\right)-\frac{c_{i} r\left(c_{i} \mid \nu\right)}{\nu}\right\} \\
& -\frac{1}{2} \sum_{y_{i} \leq k}\left\{\psi\left(\frac{\nu+1}{2}\right)-\psi\left(\frac{\nu}{2}\right)-b_{01}\left(z_{i} \mid \nu\right)-\frac{z_{i} r\left(z_{i} \mid \nu\right)}{\nu}\right\} \\
& j_{\alpha \alpha}=\sum_{y_{i} \leq k} \frac{1}{\alpha^{2}}
\end{aligned}
$$




\section{B.4 Matriz de Informação Modelo PT-CR}

\section{B.4.1 Matriz de Informação Observada}

Computando a segunda derivada parcial da função de log-verissimilhança (3.42) obtemos os elementos da matriz de informação observada $\boldsymbol{J}(\boldsymbol{\varphi})$ para o modelo PT-CR model. Temos que

$$
\begin{aligned}
& \boldsymbol{j} \boldsymbol{\gamma} \boldsymbol{\gamma}^{\top}=\alpha \sum_{i=1}^{n}\left(1-d_{i}\right)\left\{r\left(-c_{i} \mid \nu\right)-\left(\frac{\nu+1}{\nu}\right)\left(1+\frac{c_{i}^{2}}{\nu}\right)^{-1} c_{i}\right\} \\
& \times r\left(-c_{i} \mid \nu\right) \mathbf{x}_{i} \mathbf{x}_{i}^{\top} \\
& +\left(\frac{\nu+1}{\nu}\right) \sum_{i=1}^{n} d_{i}\left\{\left(1+\frac{z_{i}^{2}}{\nu}\right)^{-1}-\frac{2}{\nu}\left(1+\frac{z_{i}^{2}}{\nu}\right)^{-2} z_{i}^{2}\right\} \mathbf{x}_{i} \mathbf{x}_{i}^{\top} \\
& +(\alpha-1) \sum_{i=1}^{n} d_{i}\left\{r\left(z_{i} \mid \nu\right)+\left(\frac{\nu+1}{\nu}\right)\left(1+\frac{z_{i}^{2}}{\nu}\right)^{-1} z_{i}\right\} \\
& \times r\left(z_{i} \mid \nu\right) \mathbf{x}_{i} \mathbf{x}_{i}^{\top} \\
& \boldsymbol{j} \boldsymbol{\gamma} \sigma=-\left(\frac{\nu+1}{\nu}\right) \sum_{i=1}^{n} d_{i}\left\{\left(1+\frac{z_{i}^{2}}{\nu}\right)^{-1}-\frac{2}{\nu}\left(1+\frac{z_{i}^{2}}{\nu}\right)^{-2} z_{i}^{2}\right\} y_{i} \mathbf{x}_{i} \\
& -(\alpha-1) \sum_{i=1}^{n} d_{i}\left\{r\left(z_{i} \mid \nu\right)+\left(\frac{\nu+1}{\nu}\right)\left(1+\frac{z_{i}^{2}}{\nu}\right)^{-1} z_{i}\right\} \\
& \times r\left(z_{i} \mid \nu\right) y_{i} \mathbf{x}_{i} \\
& \boldsymbol{j} \boldsymbol{\gamma} \nu=\frac{\alpha}{2} \sum_{i=1}^{n}\left(1-d_{i}\right)\left\{\left(\psi\left(\frac{\nu+1}{2}\right)-\psi\left(\frac{\nu}{2}\right)\right)\left(1+R\left(c_{i} \mid \nu\right)\right)\right. \\
& -\frac{1}{\nu}\left(1+c_{i} r\left(c_{i} \mid \nu\right) R\left(c_{i} \mid \nu\right)\right)-R\left(c_{i} \mid \nu\right) b_{01}\left(c_{i} \mid \nu\right)-\log \left(1+\frac{c_{i}^{2}}{\nu}\right) \\
& \left.+\left(\frac{\nu+1}{\nu}\right)\left(1+\frac{c_{i}^{2}}{\nu}\right)^{-1} \frac{c_{i}^{2}}{\nu}\right\} r\left(-c_{i} \mid \nu\right) \mathbf{x}_{i} \\
& +\frac{1}{\nu^{2}} \sum_{i=1}^{n} d_{i}\left\{\left(1+\frac{z_{i}^{2}}{\nu}\right)^{-1} z_{i}-\left(\frac{\nu+1}{\nu}\right)\left(1+\frac{z_{i}^{2}}{\nu}\right)^{-2} z_{i}^{3}\right\} \mathbf{x}_{i} \\
& +\frac{\alpha-1}{2} \sum_{i=1}^{n} d_{i}\left\{\left(\frac{\nu+1}{\nu}\right)\left(1+\frac{z_{i}^{2}}{\nu}\right)^{-1} \frac{z_{i}^{2}}{\nu}+b_{01}\left(z_{i} \mid \nu\right)\right. \\
& \left.-\log \left(1+\frac{z_{i}^{2}}{\nu}\right)-\frac{1}{\nu}\left(1-z_{i} r\left(z_{i} \mid \nu\right)\right)\right\} r\left(z_{i} \mid \nu\right) \mathbf{x}_{i} \\
& \boldsymbol{j} \gamma \alpha=\sum_{i=1}^{n}\left(1-d_{i}\right) r\left(-c_{i} \mid \nu\right) \mathbf{x}_{i}+\sum_{i=1}^{n} d_{i} r\left(z_{i} \mid \nu\right) \mathbf{x}_{i}
\end{aligned}
$$




$$
\begin{aligned}
& j \sigma \sigma=\sum_{i=1}^{n} d_{i}\left\{\frac{1}{\sigma^{2}}+\left(\frac{\nu+1}{\nu}\right)\left[\left(1+\frac{z_{i}^{2}}{\nu}\right)^{-1}-\frac{2}{\nu}\left(1+\frac{z_{i}^{2}}{\nu}\right)^{-2} z_{i}^{2}\right] y_{i}^{2}\right\} \\
& +(\alpha-1) \sum_{i=1}^{n} I_{i}\left\{\left(\frac{\nu+1}{\nu}\right)\left(1+\frac{z_{i}^{2}}{\nu}\right)^{-1} z_{i}+r\left(z_{i} \mid \nu\right)\right\} r\left(z_{i} \mid \nu\right) y_{i}^{2} \\
& j \sigma \nu=-\frac{1}{\nu^{2}} \sum_{i=1}^{n} d_{i}\left\{\left(1+\frac{z_{i}^{2}}{\nu}\right)^{-1} z_{i}-\left(\frac{\nu+1}{\nu}\right)\left(1+\frac{z_{i}^{2}}{\nu}\right)^{-2} z_{i}^{3}\right\} y_{i} \\
& -\frac{\alpha-1}{2} \sum_{i=1}^{n} d_{i}\left\{\left(\frac{\nu+1}{\nu}\right)\left(1+\frac{z_{i}^{2}}{\nu}\right)^{-1} \frac{z_{i}^{2}}{\nu}+b_{01}\left(z_{i} \mid \nu\right)\right. \\
& \left.-\log \left(1+\frac{z_{i}^{2}}{\nu}\right)-\frac{1}{\nu}\left(1-z_{i} r\left(z_{i} \mid \nu\right)\right)\right\} r\left(z_{i} \mid \nu\right) y_{i} \\
& j \sigma \alpha=-\sum_{i=1}^{n} d_{i} r\left(z_{i} \mid \nu\right) y_{i} \\
& j \nu \nu=+\frac{\alpha}{4} \sum_{i=1}^{n}\left(1-d_{i}\right)\left\{\psi\left(\frac{\nu+1}{2}\right)-\psi\left(\frac{\nu}{2}\right)-b_{01}\left(c_{i} \mid \nu\right)\right. \\
& \left.-\frac{c_{i}}{\nu} r\left(c_{i} \mid \nu\right)\right\}^{2} R\left(c_{i} \mid \nu\right)\left(1+R\left(c_{i} \mid \nu\right)\right) \\
& -\frac{\alpha}{4} \sum_{i=1}^{n}\left(1-d_{i}\right)\left\{\psi_{1}\left(\frac{\nu}{2}\right)-\psi_{1}\left(\frac{\nu+1}{2}\right)-\frac{2}{\nu(\nu+1)}+b_{01}^{2}\left(c_{i} \mid \nu\right)\right. \\
& -\frac{1}{\nu} b_{01}\left(c_{i} \mid \nu\right)+\frac{1}{\nu}\left(2 b_{01}\left(c_{i} \mid \nu\right)-\frac{\nu+3}{\nu(\nu+1)}\right) c_{i} r\left(c_{i} \mid \nu\right) \\
& +\frac{1}{\nu+2} \frac{T\left(c_{2 i} \mid \nu+2\right)}{T\left(c_{i} \mid \nu\right)} b_{21}\left(c_{2 i} \mid \nu+2\right)-b_{02}\left(c_{i} \mid \nu\right) \\
& +\frac{1}{\nu}\left[\left(\frac{\nu+1}{\nu}\right)\left(1+\frac{c_{i}^{2}}{\nu}\right)^{-1} \frac{c_{i}^{2}}{\nu}-\log \left(1+\frac{c_{i}^{2}}{\nu}\right)+\frac{c_{i}}{\nu} r\left(c_{i} \mid \nu\right)\right] \\
& \left.\times c_{i} r\left(c_{i} \mid \nu\right)\right\} R\left(c_{i} \mid \nu\right) \\
& -\frac{1}{4} \sum_{i=1}^{n} d_{i}\left\{\psi_{1}\left(\frac{\nu+1}{2}\right)-\psi_{1}\left(\frac{\nu}{2}\right)+\frac{2}{\nu^{2}}-\frac{4}{\nu^{3}}\left(1+\frac{z_{i}^{2}}{\nu}\right)^{-1} z_{i}^{2}\right. \\
& \left.+\frac{2}{\nu^{3}}\left(\frac{\nu+1}{\nu}\right)\left(1+\frac{z_{i}^{2}}{\nu}\right)^{-2} z_{i}^{4}\right\} \\
& +\frac{\alpha-1}{4} \sum_{i=1}^{n} d_{i}\left\{\psi_{1}\left(\frac{\nu}{2}\right)-\psi_{1}\left(\frac{\nu+1}{2}\right)-\frac{2}{\nu(\nu+1)}+b_{01}^{2}\left(z_{i} \mid \nu\right)\right. \\
& -\frac{1}{\nu} b_{01}\left(z_{i} \mid \nu\right)+\frac{1}{\nu}\left(2 b_{01}\left(z_{i} \mid \nu\right)-\frac{\nu+3}{\nu(\nu+1)}\right) z_{i} r\left(z_{i} \mid \nu\right) \\
& +\frac{1}{\nu+2} \frac{T\left(z_{2 i} \mid \nu+2\right)}{T\left(z_{i} \mid \nu\right)} b_{21}\left(z_{2 i} \mid \nu+2\right)-b_{02}\left(z_{i} \mid \nu\right) \\
& +\frac{1}{\nu}\left[\left(\frac{\nu+1}{\nu}\right)\left(1+\frac{z_{i}^{2}}{\nu}\right)^{-1} \frac{z_{i}^{2}}{\nu}\right. \\
& \left.\left.-\log \left(1+\frac{z_{i}^{2}}{\nu}\right)+\frac{z_{i}}{\nu} r\left(z_{i} \mid \nu\right)\right] z_{i} r\left(z_{i} \mid \nu\right)\right\}
\end{aligned}
$$




$$
\begin{aligned}
j \nu \alpha=- & \frac{1}{2} \sum_{i=1}^{n}\left(1-d_{i}\right)\left\{\psi\left(\frac{\nu+1}{2}\right)-\psi\left(\frac{\nu}{2}\right)-b_{01}\left(c_{i} \mid \nu\right)-\frac{c_{i}}{\nu} r\left(c_{i} \mid \nu\right)\right\} \\
& \times R\left(c_{i} \mid \nu\right)+\frac{1}{2} \sum_{i=1}^{n} d_{i}\left\{\psi\left(\frac{\nu+1}{2}\right)-\psi\left(\frac{\nu}{2}\right)-b_{01}\left(z_{i} \mid \nu\right)\right. \\
& \left.\quad-\frac{z_{i}}{\nu} r\left(z_{i} \mid \nu\right)\right\} \\
j \alpha \alpha=- & \sum_{i=1}^{n} \frac{d_{i}}{\alpha^{2}}
\end{aligned}
$$

em que $\psi_{1}(x)=\frac{d}{d x} \psi(x)$ é a função trigama.

\section{B.4.2 Matriz de Informação Esperada}

A matriz de informação esperada $\boldsymbol{I}(\boldsymbol{\varphi})$ é obtida tomando $\boldsymbol{I}(\boldsymbol{\varphi})=\mathrm{E}[\boldsymbol{J}(\boldsymbol{\varphi})]$, que envolve o computo de momentos truncados que não têm forma fechada e deven ser obtidos numéricamente.

Temos que os elementos $I_{\boldsymbol{\varphi}_{i} \boldsymbol{\varphi}_{j}}$ oda matriz de informação esperada são dados por

$$
\begin{aligned}
\boldsymbol{I}_{\boldsymbol{\gamma} \boldsymbol{\gamma}}= & \sum_{i=1}^{n}\left\{r\left(-c_{i} \mid \nu\right)-\left(\frac{\nu+1}{\nu}\right)\left(1+\frac{c_{i}}{\nu}\right)^{-1} c_{i}\right\} \phi_{\alpha}\left(-c_{i} \mid \nu\right) \mathbf{x}_{i} \mathbf{x}_{i}^{\top} \\
& +\left(\frac{\nu+1}{\nu}\right) \sum_{i=1}^{n}\left\{a_{020}\left(-c_{i} \mid \nu\right)-\frac{2}{\nu} a_{042}\left(-c_{i} \mid \nu\right)\right\} \mathbf{x}_{i} \mathbf{x}_{i}^{\top} \\
& +(\alpha-1) t(0 \mid \nu) \sum_{i=1}^{n}\left\{\left(\frac{\nu+1}{\nu}\right) a_{121}\left(-c_{i} \mid \nu\right)\right. \\
& \left.+t(0 \mid \nu) a_{200}\left(-c_{i} \mid \nu\right)\right\} \mathbf{x}_{i} \mathbf{x}_{i}^{\top} \\
\boldsymbol{I}_{\boldsymbol{\gamma} \sigma}= & -\frac{1}{\sigma}\left(\frac{\nu+1}{\nu}\right) \sum_{i=1}^{n}\left\{a_{021}\left(-c_{i} \mid \nu\right)+c_{i} a_{020}\left(-c_{i} \mid \nu\right)\right\} \mathbf{x}_{i} \\
& +\frac{2}{\sigma}\left(\frac{\nu+1}{\nu^{2}}\right) \sum_{i=1}^{n}\left\{a_{043}\left(-c_{i} \mid \nu\right)+c_{i} a_{042}\left(-c_{i} \mid \nu\right)\right\} \mathbf{x}_{i} \\
& -(t(0 \mid \nu))^{2}\left(\frac{\alpha-1}{\sigma}\right) \sum_{i=1}^{n}\left\{a_{201}\left(-c_{i} \mid \nu\right)+c_{i} a_{200}\left(-c_{i} \mid \nu\right)\right\} \mathbf{x}_{i} \\
& -t(0 \mid \nu)\left(\frac{\alpha-1}{\sigma}\right)\left(\frac{\nu+1}{\nu}\right) \sum_{i=1}^{n}\left\{a_{122}\left(-c_{i} \mid \nu\right)\right. \\
& \left.+c_{i} a_{121}\left(-c_{i} \mid \nu\right)\right\} \mathbf{x}_{i}
\end{aligned}
$$




$$
\begin{aligned}
& \boldsymbol{I}_{\gamma \nu}=\frac{\alpha}{2} \sum_{i=1}^{n}\left\{\left(\psi\left(\frac{\nu+1}{2}\right)-\psi\left(\frac{\nu}{2}\right)\right)\left(1+R\left(c_{i} \mid \nu\right)\right)\right. \\
& -\frac{1}{\nu}\left(1+c_{i} r\left(c_{i} \mid \nu\right) R\left(c_{i} \mid \nu\right)\right)-R\left(c_{i} \mid \nu\right) b_{01}\left(c_{i} \mid \nu\right)-\log \left(1+\frac{c_{i}^{2}}{\nu}\right) \\
& \left.+\left(\frac{\nu+1}{\nu}\right)\left(1+\frac{c_{i}^{2}}{\nu}\right)^{-1} \frac{c_{i}^{2}}{\nu}\right\} \phi_{\alpha}\left(-c_{i} \mid \nu\right) \mathbf{x}_{i} \\
& +\frac{1}{\nu^{2}} \sum_{i=1}^{n}\left\{a_{021}\left(-c_{i} \mid \nu\right)-\left(\frac{\nu+1}{\nu}\right) a_{043}\left(-c_{i} \mid \nu\right)\right\} \mathbf{x}_{i} \\
& +t(0 \mid \nu)\left(\frac{\alpha-1}{2}\right) \sum_{i=1}^{n}\left\{\left(\frac{\nu+1}{\nu}\right) a_{122}\left(-c_{i} \mid \nu\right)-a_{10}\left(-c_{i} \mid \nu\right)\right. \\
& \left.+b_{110}\left(-c_{i} \mid \nu\right)-\frac{1}{\nu}\left(a_{100}\left(-c_{i} \mid \nu\right)-t(0 \mid \nu) a_{201}\left(-c_{i} \mid \nu\right)\right)\right\} \mathbf{x}_{i} \\
& \boldsymbol{I}_{\boldsymbol{\gamma} \alpha}=\frac{1}{\alpha} \sum_{i=1}^{n} \phi_{\alpha}\left(-c_{i} \mid \nu\right) \mathbf{x}_{i}+t(0 \mid \nu) \sum_{i=1}^{n} a_{100}\left(-c_{i} \mid \nu\right) \mathbf{x}_{i} \\
& I_{\sigma \sigma}=\frac{1}{\sigma^{2}} \sum_{i=1}^{n}\left\{1+\left(\frac{\nu+1}{\nu}\right)\left[a_{022}\left(-c_{i} \mid \nu\right)+2 c_{i} a_{021}\left(-c_{i} \mid \nu\right)\right.\right. \\
& +c_{i}^{2} a_{020}\left(-c_{i} \mid \nu\right)-\frac{2}{\nu}\left(a_{044}\left(-c_{i} \mid \nu\right)+2 c_{i} a_{043}\left(-c_{i} \mid \nu\right)\right. \\
& \left.\left.\left.+c_{i}^{2} a_{042}\left(-c_{i} \mid \nu\right)\right)\right]\right\} \\
& +t(0 \mid \nu)\left(\frac{\alpha-1}{\sigma^{2}}\right) \sum_{i=1}^{n}\left\{( \frac { \nu + 1 } { \nu } ) \left[a_{123}\left(-c_{i} \mid \nu\right)+2 c_{i} a_{122}\left(-c_{i} \mid \nu\right)\right.\right. \\
& \left.+c_{i}^{2} a_{121}\left(-c_{i} \mid \nu\right)\right]+t(0 \mid \nu)\left[a_{202}\left(-c_{i} \mid \nu\right)+2 c_{i} a_{201}\left(-c_{i} \mid \nu\right)\right. \\
& \left.\left.+c_{i}^{2} a_{200}\left(-c_{i} \mid \nu\right)\right]\right\} \\
& I_{\sigma \nu}=-\frac{1}{\sigma \nu^{2}} \sum_{i=1}^{n}\left\{a_{022}\left(-c_{i} \mid \nu\right)+c_{i} a_{021}\left(-c_{i} \mid \nu\right)\right. \\
& \left.-\left(\frac{\nu+1}{\nu}\right)\left[a_{044}\left(-c_{i} \mid \nu\right)+c_{i} a_{043}\left(-c_{i} \mid \nu\right)\right]\right\}\left\{T\left(-c_{i} \mid \nu\right)\right\}^{\alpha} \\
& -t(0 \mid \nu)\left(\frac{\alpha-1}{2 \sigma}\right) \sum_{i=1}^{n}\left\{\left(\frac{\nu+1}{\nu^{2}}\right)\left[a_{123}\left(-c_{i} \mid \nu\right)+c_{i} a_{122}\left(-c_{i} \mid \nu\right)\right]\right. \\
& -a_{11}\left(-c_{i} \mid \nu\right)-c_{i} a_{10}\left(-c_{i} \mid \nu\right)+b_{111}\left(-c_{i} \mid \nu\right)+c_{i} b_{110}\left(-c_{i} \mid \nu\right) \\
& -\frac{1}{\nu}\left[a_{101}\left(-c_{i} \mid \nu\right)+c_{i} a_{100}\left(-c_{i} \mid \nu\right)\right] \\
& \left.+\frac{1}{\nu}\left[t(0 \mid \nu) a_{202}\left(-c_{i} \mid \nu\right)+c_{i} t(0 \mid \nu) a_{201}\left(-c_{i} \mid \nu\right)\right]\right\} \\
& I_{\sigma \alpha}=-\frac{t(0 \mid \nu)}{\sigma} \sum_{i=1}^{n}\left\{a_{101}\left(-c_{i} \mid \nu\right)+c_{i} a_{100}\left(-c_{i} \mid \nu\right)\right\}
\end{aligned}
$$




$$
\begin{aligned}
& I_{\nu \nu}=\frac{\alpha}{4} \sum_{i=1}^{n}\left\{\psi\left(\frac{\nu+1}{2}\right)-\psi\left(\frac{\nu}{2}\right)-b_{01}\left(c_{i} \mid \nu\right)-\frac{c_{i}}{\nu} r\left(c_{i} \mid \nu\right)\right\}^{2} \\
& \times R\left(c_{i} \mid \nu\right)\left(1+R\left(c_{i} \mid \nu\right)\right)\left\{T\left(-c_{i} \mid \nu\right)\right\}^{\alpha} \\
& -\frac{\alpha}{4} \sum_{i=1}^{n}\left\{\psi_{1}\left(\frac{\nu}{2}\right)-\psi_{1}\left(\frac{\nu+1}{2}\right)-\frac{2}{\nu(\nu+1)}+b_{01}^{2}\left(c_{i} \mid \nu\right)\right. \\
& -\frac{1}{\nu} b_{01}\left(c_{i} \mid \nu\right)+\frac{1}{\nu}\left(2 b_{01}\left(c_{i} \mid \nu\right)-\frac{\nu+3}{\nu(\nu+1)}\right) c_{i} r\left(c_{i} \mid \nu\right) \\
& -b_{02}\left(c_{i} \mid \nu\right)+\frac{T\left(c_{2 i} \mid \nu+2\right) b_{21}\left(c_{2 i} \mid \nu+2\right)}{(\nu+2) T\left(c_{2 i} \mid \nu\right)} \\
& +\frac{1}{\nu}\left[\left(\frac{\nu+1}{\nu}\right)\left(1+\frac{c_{i}^{2}}{\nu}\right)^{-1} \frac{c_{i}^{2}}{\nu}\right. \\
& \left.\left.-\log \left(1+\frac{c_{i}^{2}}{\nu}\right)+\frac{c_{i}}{\nu} r\left(c_{i} \mid \nu\right)\right] c_{i} r\left(c_{i} \mid \nu\right)\right\} R\left(c_{i} \mid \nu\right)\left\{T\left(-c_{i} \mid \nu\right)\right\}^{\alpha} \\
& -\frac{1}{4} \sum_{i=1}^{n}\left\{\psi_{1}\left(\frac{\nu+1}{2}\right)-\psi_{1}\left(\frac{\nu}{2}\right)+\frac{2}{\nu^{2}}-\frac{4 a_{022}\left(-c_{i} \mid \nu\right)}{\nu^{3}\left(1-\left\{T\left(-c_{i} \mid \nu\right)\right\}^{\alpha}\right)}\right. \\
& \left.+\frac{2}{\nu^{3}}\left(\frac{\nu+1}{\nu}\right) \frac{a_{044}\left(-c_{i} \mid \nu\right)}{\left(1-\left\{T\left(-c_{i} \mid \nu\right)\right\}^{\alpha}\right)}\right\}\left(1-\left\{T\left(-c_{i} \mid \nu\right)\right\}^{\alpha}\right) \\
& +\frac{\alpha-1}{4} \sum_{i=1}^{n}\left\{\psi_{1}\left(\frac{\nu}{2}\right)-\psi_{1}\left(\frac{\nu+1}{2}\right)-\frac{2}{\nu(\nu+1)}\right. \\
& +\frac{b_{020}\left(-c_{i} \mid \nu\right)}{1-\left\{T\left(-c_{i} \mid \nu\right)\right\}^{\alpha}}-\frac{b_{010}\left(-c_{i} \mid \nu\right)}{\nu\left(1-\left\{T\left(-c_{i} \mid \nu\right)\right\}^{\alpha}\right)} \\
& +\frac{1}{\nu}\left[\frac{2 t(0 \mid \nu) b_{111}\left(-c_{i} \mid \nu\right)}{1-\left\{T\left(-c_{i} \mid \nu\right)\right\}^{\alpha}}-\frac{\nu+3}{\nu(\nu+1)} \frac{t(0 \mid \nu) a_{101}\left(-c_{i} \mid \nu\right)}{\left(1-\left\{T\left(-c_{i} \mid \nu\right)\right\}^{\alpha}\right)}\right] \\
& -\mathrm{E}\left[b_{02}\left(z_{i} \mid \nu\right)\right]+\left(\frac{1}{\nu+2}\right) \mathrm{E}\left[\frac{T\left(z_{2 i} \mid \nu+2\right)}{T\left(z_{i} \mid \nu\right)} b_{21}\left(z_{2 i} \mid \nu+2\right)\right] \\
& +\frac{t(0 \mid \nu)}{\nu\left(1-\left\{T\left(-c_{i} \mid \nu\right)\right\}^{\alpha}\right)}\left[\left(\frac{\nu+1}{\nu^{2}}\right) a_{123}\left(-c_{i} \mid \nu\right)-a_{11}\left(-c_{i} \mid \nu\right)\right. \\
& \left.\left.+\frac{1}{\nu} t(0 \mid \nu) a_{202}\left(-c_{i} \mid \nu\right)\right]\right\}\left(1-\left\{T\left(-c_{i} \mid \nu\right)\right\}^{\alpha}\right) \\
& I_{\nu \alpha}=\frac{1}{2 \alpha} \sum_{i=1}^{n}\left\{\psi\left(\frac{\nu+1}{2}\right)-\psi\left(\frac{\nu}{2}\right)-b_{01}\left(c_{i} \mid \nu\right)-\frac{c_{i}}{\nu} r\left(c_{i} \mid \nu\right)\right\} \\
& \times\left(r\left(-c_{i} \mid \nu\right)\right)^{-1} \phi_{\alpha}\left(-c_{i} \mid \nu\right) \\
& -\frac{1}{2} \sum_{i=1}^{n}\left\{\psi\left(\frac{\nu+1}{2}\right)-\psi\left(\frac{\nu}{2}\right)-\frac{b_{010}\left(-c_{i} \mid \nu\right)}{1-\left\{T\left(-c_{i} \mid \nu\right)\right\}^{\alpha}}\right. \\
& \left.-\frac{t(0 \mid \nu) a_{101}\left(-c_{i} \mid \nu\right)}{\nu\left(1-\left\{T\left(-c_{i} \mid \nu\right)\right\}^{\alpha}\right)}\right\}\left(1-\left\{T\left(-c_{i} \mid \nu\right)\right\}^{\alpha}\right) \\
& I_{\alpha \alpha}=\frac{1}{\alpha^{2}} \sum_{i=1}^{n}\left(1-\left\{T\left(-c_{i} \mid \nu\right)\right\}^{\alpha}\right)
\end{aligned}
$$

em que $b_{01}, b_{02}$, e $b_{21}$ são dados por (3.29) e devem ser obtidos numéricamente. Os componentes $a_{k m n}, a_{k n}$ and $b_{k m n}$, são dados na Proposição 4 deven ser computados numéricamente também. 


\section{B.5 Função Escore e Matriz de Informação Observada no Modelo PNLMM}

Apresentamos nesta seção as expressões para elementos da função escore e a matriz de informação observada do modelo de regressão linear misto normal-potência desenvolvido na Capítulo 3 .

\section{B.5.1 Função Escore}

Considerando a fdp da distribuição normal-potência (2.13) e considerando o modelo de regressão normal-potência com intercepto aleatório potência-normal dado em (4.2), o logaritmo da função de verossimilhança aproximada (4.7) pode ser expresso como

$$
\ell(\boldsymbol{\theta} ; \mathbf{Y}) \approx \sum_{i=1}^{n} \log B_{i}
$$

em que

$$
B_{i}=\sum_{k=1}^{Q} \frac{\omega_{k}}{\sqrt{\pi}} A_{i}
$$

e

$$
\begin{aligned}
A_{i}= & \exp \left(m_{i} \log \left(\alpha_{e} / \eta_{e}\right)+\sum_{j=1}^{m_{i}} \log \phi\left(z_{i j}\right)\right. \\
& \left.+\left(\alpha_{e}-1\right) \sum_{j=1}^{m_{i}} \log \Phi\left(z_{i j}\right)+\log \left(\alpha_{g}\right)+\left(\alpha_{g}-1\right) \log \Phi\left(\sqrt{2} u_{k}\right)\right)
\end{aligned}
$$

com $z_{i j}=\left(y_{i j}-\mathbf{x}_{i j}^{\top} \boldsymbol{\beta}-\sqrt{2} \eta_{g} u_{k}\right) / \eta_{e}$. A função escore é obtida diferenciando o logaritmo da função de verossimlhança (B.3) em relação a cada um dos parâmetros. Definindo $v_{i j}=\frac{\phi\left(z_{i j}\right)}{\Phi\left(z_{i j}\right)}$, obtemos que $\boldsymbol{U}(\boldsymbol{\theta})=\left(\boldsymbol{U}_{\boldsymbol{\beta}}^{\top}(\boldsymbol{\theta}), U_{\eta_{e}}(\boldsymbol{\theta}), U_{\eta_{g}}(\boldsymbol{\theta}), U_{\alpha_{e}}(\boldsymbol{\theta}), U_{\alpha_{g}}(\boldsymbol{\theta})\right)^{\top}, \mathrm{com}$

$$
\begin{aligned}
\boldsymbol{U}_{\boldsymbol{\beta}}(\boldsymbol{\theta}) & =\sum_{i=1}^{n} \frac{\mathbf{X}_{i}^{\top} \mathbf{D}_{i 1}}{B_{i}}, & U_{\eta_{e}}(\boldsymbol{\theta}) & =\sum_{i=1}^{n} \frac{D_{i 2}}{B_{i}}, \\
U_{\alpha_{e}}(\boldsymbol{\theta}) & =\sum_{i=1}^{n} \frac{D_{i 4}}{B_{i}}, & U_{\alpha_{g}}(\boldsymbol{\theta}) & =\sum_{i=1}^{n} \frac{D_{i 5}}{B_{i}}
\end{aligned}
$$

em que $\mathbf{X}_{i}$ é uma matriz de dimensão $m_{i} \times p$ com linhas $\mathbf{x}_{i j}^{\top}=\left(x_{i j 1}, \ldots, x_{i j p}\right)$, para $j=1, \ldots, m_{i}$, e

$$
\mathbf{D}_{i 1}=\sum_{k=1}^{Q} \frac{\omega_{k}}{\sqrt{\pi}} A_{i} \mathbf{P}_{i 1}^{\top} \mathbf{E}_{i 1}
$$

$\operatorname{com} \mathbf{P}_{i 1}=\operatorname{diag}\left\{1 / \eta_{e}, \ldots, 1 / \eta_{e}\right\}$ uma matriz $m_{i} \times m_{i}$, e $\mathbf{E}_{i 1}=\left(e_{i 11}, \ldots, e_{i m_{i} 1}\right)^{\top}$ um vetor de dimensão $m_{i} \times 1$ de elementos $e_{i j 1}=z_{i j}-\left(\alpha_{e}-1\right) v_{i j}$. A quantidade $D_{i 2}$ em $U_{\eta_{e}}(\boldsymbol{\theta})$ é dada por

$$
D_{i 2}=\sum_{k=1}^{Q} \frac{\omega_{k}}{\sqrt{\pi}} A_{i} \mathbf{P}_{i 1}^{* \top} \mathbf{E}_{i 2},
$$


em que $\mathbf{P}_{i 1}^{*}=\left(1 / \eta_{e}, \ldots, 1 / \eta_{e}\right)^{\top}$ é um vetor de dimensão $m_{i} \times 1$, e $\mathbf{E}_{i 2}=\left(e_{i 12}, \ldots, e_{i m_{i} 2}\right)^{\top}$ é um vetor de dimensão $m_{i} \times 1$ de elementos $e_{i j 2}=-1+z_{i j}^{2}-\left(\alpha_{e}-1\right) z_{i j} v_{i j}$. A quantidade $D_{i 3} \mathrm{em}$ $U_{\eta_{g}}(\boldsymbol{\theta})$ é dada por

$$
D_{i 3}=\sum_{k=1}^{Q} \frac{\sqrt{2} \omega_{k} u_{k}}{\sqrt{\pi}} A_{i} \mathbf{P}_{i 1}^{* \top} \mathbf{E}_{i 1},
$$

e a quantidade $D_{i 4}$ in $U_{\alpha_{e}}(\boldsymbol{\theta})$ é dada por

$$
D_{i 4}=\sum_{k=1}^{Q} \frac{\omega_{k}}{\sqrt{\pi}} A_{i} \mathbf{1}_{m_{i}}^{\top} \mathbf{E}_{i 3},
$$

em que $\mathbf{E}_{i 3}=\left(e_{i j 3}, \ldots, e_{i m_{i} 3}\right)^{\top}$ é um vetor de dimensão $m_{i} \times 1$ com elementos $e_{i j 3}=\frac{1}{\alpha_{e}}+$ $\log \Phi\left(z_{i j}\right)$ e $\mathbf{1}_{m_{i}}$ é um vetor de uns de dimensão $m_{i} \times 1$. Finalmente a quantidade $D_{i 5}$ em $U_{\alpha_{g}}(\boldsymbol{\theta})$ é dada por

$$
D_{i 5}=\sum_{k=1}^{Q} \frac{\omega_{k}}{\sqrt{\pi}} A_{i} Q_{4}
$$

em que $Q_{4}=\frac{1}{\alpha_{g}}+\log \Phi\left(\sqrt{2} u_{k}\right)$.

\section{B.5.2 Matriz de Informação Observada}

A matriz de informação observada $\boldsymbol{J}(\boldsymbol{\theta})$ de ordem $(p+5) \times(p+5)$ para o modelo de regressão normal-potência com intercepto aleatório distribuído normal-potência é dada por:

$$
\boldsymbol{J}(\boldsymbol{\theta})=\left[\begin{array}{lllll}
\boldsymbol{j}_{\boldsymbol{\beta} \boldsymbol{\beta}} & \boldsymbol{j}_{\boldsymbol{\beta} \eta_{e}} & \boldsymbol{j}_{\boldsymbol{\beta} \eta_{g}} & \boldsymbol{j}_{\boldsymbol{\beta} \alpha_{e}} & \boldsymbol{j}_{\boldsymbol{\beta} \alpha_{g}} \\
\boldsymbol{j}_{\eta_{e} \boldsymbol{\beta}} & j_{\eta_{e} \eta_{e}} & j_{\eta_{e} \eta_{g}} & j_{\eta_{e} \alpha_{e}} & j_{\eta_{e} \alpha_{g}} \\
\boldsymbol{j}_{\eta_{g} \boldsymbol{\beta}} & j_{\eta_{g} \eta_{\varepsilon}} & j_{\eta_{g} \eta_{g}} & j_{\eta_{g} \alpha_{e}} & j_{\eta_{g} \alpha_{g}} \\
\boldsymbol{j}_{\alpha_{e} \boldsymbol{\beta}} & j_{\alpha_{e} \eta_{e}} & j_{\alpha_{e} \eta_{g}} & j_{\alpha_{e} \alpha_{e}} & j_{\alpha_{e} \alpha_{g}} \\
\boldsymbol{j}_{\alpha_{g} \boldsymbol{\beta}} & j_{\alpha_{g} \eta_{e}} & j_{\alpha_{g} \eta_{g}} & j_{\alpha_{g} \alpha_{e}} & j_{\alpha_{g} \alpha_{g}}
\end{array}\right]
$$

em que $\boldsymbol{j}_{\boldsymbol{\theta}_{j} \boldsymbol{\theta}_{k}}$ é obtida como

$$
\begin{aligned}
\boldsymbol{j}_{\boldsymbol{\theta}_{j} \boldsymbol{\theta}_{k}} & =-\sum_{i=1}^{n} \frac{\partial \boldsymbol{U}_{k}(\boldsymbol{\theta})}{\partial \boldsymbol{\theta}_{j}} \\
& =-\sum_{i=1}^{n} \frac{\partial}{\partial \boldsymbol{\theta}_{j}}\left(\frac{1}{B_{i}} \frac{\partial B_{i}}{\partial \boldsymbol{\theta}_{k}}\right) \\
& =-\sum_{i=1}^{n}\left(\frac{1}{B_{i}} \frac{\partial^{2} B_{i}}{\partial \boldsymbol{\theta}_{j} \partial \boldsymbol{\theta}_{k}}-\frac{1}{B_{i}^{2}} \frac{\partial B_{i}}{\partial \boldsymbol{\theta}_{j}} \frac{\partial B_{i}}{\partial \boldsymbol{\theta}_{k}}\right)
\end{aligned}
$$


Baseados em (B.4) obtemos as derivadas apresentadas a seguir

$$
\begin{array}{rlrl}
\boldsymbol{j}_{\boldsymbol{\beta}^{\top} \boldsymbol{\beta}} & =\sum_{i=1}^{n} \mathbf{X}_{i}^{\top}\left(\frac{\mathbf{D}_{i 1} \mathbf{D}_{i 1}^{\top}}{B_{i}^{2}}-\frac{\mathbf{D}_{i 6}}{B_{i}}\right) \mathbf{X}_{i}, & j_{\eta_{g} \alpha_{e}} & =\sum_{i=1}^{n}\left(\frac{D_{i 3} D_{i 4}}{B_{i}^{2}}-\frac{D_{i 16}}{B_{i}}\right), \\
\boldsymbol{j}_{\boldsymbol{\beta} \eta_{e}} & =\sum_{i=1}^{n} \mathbf{X}_{i}^{\top}\left(\frac{\mathbf{D}_{i 1} D_{i 2}}{B_{i}^{2}}-\frac{\mathbf{D}_{i 7}}{B_{i}}\right), & j_{\eta_{g} \alpha_{g}} & =\sum_{i=1}^{n}\left(\frac{D_{i 3} D_{i 5}}{B_{i}^{2}}-\frac{D_{i 17}}{B_{i}}\right), \\
\boldsymbol{j}_{\boldsymbol{\beta} \eta_{g}} & =\sum_{i=1}^{n} \mathbf{X}_{i}^{\top}\left(\frac{\mathbf{D}_{i 1} D_{i 3}}{B_{i}^{2}}-\frac{\mathbf{D}_{i 8}}{B_{i}}\right), & j_{\alpha_{e} \alpha_{e}}=\sum_{i=1}^{n}\left(\frac{D_{i 4}^{2}}{B_{i}^{2}}-\frac{D_{i 18}}{B_{i}}\right), \\
\boldsymbol{j}_{\boldsymbol{\beta} \alpha_{e}}=\sum_{i=1}^{n} \mathbf{X}_{i}^{\top}\left(\frac{\mathbf{D}_{i 1} D_{i 4}}{B_{i}^{2}}-\frac{\mathbf{D}_{i 9}}{B_{i}}\right), & j_{\alpha_{e} \alpha_{g}}=\sum_{i=1}^{n}\left(\frac{D_{i 4} D_{i 5}}{B_{i}^{2}}-\frac{D_{i 19}}{B_{i}}\right), \\
\boldsymbol{j}_{\boldsymbol{\beta} \alpha_{g}}=\sum_{i=1}^{n} \mathbf{X}_{i}^{\top}\left(\frac{\mathbf{D}_{i 1} D_{i 5}}{B_{i}^{2}}-\frac{\mathbf{D}_{i 10}}{B_{i}}\right), & j_{\alpha_{g} \alpha_{g}}=\sum_{i=1}^{n}\left(\frac{D_{i 5}^{2}}{B_{i}^{2}}-\frac{D_{i 20}}{B_{i}}\right), \\
j_{\eta_{e} \eta_{e}}=\sum_{i=1}^{n}\left(\frac{D_{i 2}^{2}}{\left.B_{i}^{2}-\frac{D_{i 11}}{B_{i}}\right),}\right. & j_{\eta_{e} \alpha_{g}}=\sum_{i=1}^{n}\left(\frac{D_{i 2} D_{i 5}}{B_{i}^{2}}-\frac{D_{i 14}}{B_{i}}\right), \\
j_{\eta_{e} \eta_{g}}=\sum_{i=1}^{n}\left(\frac{D_{i 2} D_{i 3}}{B_{i}^{2}}-\frac{D_{i 12}}{B_{i}}\right), & j_{\eta_{g} \eta_{g}}=\sum_{i=1}^{n}\left(\frac{D_{i 3}^{2}}{B_{i}^{2}}-\frac{D_{i 15}}{B_{i}}\right), \\
j_{\eta_{e} \alpha_{e}}=\sum_{i=1}^{n}\left(\frac{D_{i 2} D_{i 4}}{B_{i}^{2}}-\frac{D_{i 13}}{B_{i}}\right) . &
\end{array}
$$

em que $B_{i}, \mathbf{D}_{i 1}, D_{i 2}, D_{i 3}, D_{i 4}$ e $D_{i 5}$ são definidos como acima, e nós definimos também

$$
\mathbf{D}_{i 6}=\sum_{k=1}^{Q} \frac{\omega_{k}}{\sqrt{\pi}} A_{i} \mathbf{P}_{i 1}\left(\mathbf{E}_{i 1} \mathbf{E}_{i 1}^{\top}+\mathbf{R}_{i 1}\right) \mathbf{P}_{i 1},
$$

$\operatorname{com} \mathbf{R}_{i 1}=\operatorname{diag}\left\{r_{i 11}, \ldots, r_{i m_{i} 1}\right\}$ uma matriz $m_{i} \times m_{i}$ de elementos $r_{i j 1}=-1-\left(\alpha_{e}-1\right)\left(z_{i j} v_{i j}+v_{i j}^{2}\right)$. A matriz $\mathbf{D}_{i 7}$ é definida por

$$
\mathbf{D}_{i 7}=\sum_{k=1}^{Q} \frac{\omega_{k}}{\sqrt{\pi}} A_{i} \mathbf{P}_{i 1}\left(\mathbf{E}_{i 1} \mathbf{E}_{i 2}^{\top}+\mathbf{R}_{i 2}\right) \mathbf{P}_{i 1}^{*},
$$

$\operatorname{com} \mathbf{R}_{i 2}=\operatorname{diag}\left\{r_{i 12}, \ldots, r_{i m_{i} 2}\right\}$ uma matriz $m_{i} \times m_{i}$ de elementos $r_{i j 2}=-2 z_{i j}-\left(\alpha_{e}-1\right)\left(-v_{i j}+\right.$ $z_{i j}^{2} v_{i j}+z_{i j} v_{i j}^{2}$ ). As matrizes $\mathbf{D}_{i 8}$ e $\mathbf{D}_{i 9}$ são dadas por

$$
\begin{gathered}
\mathbf{D}_{i 8}=\sum_{k=1}^{Q} \frac{\sqrt{2} u_{k} \omega_{k}}{\sqrt{\pi}} A_{i} \mathbf{P}_{i 1}\left(\mathbf{E}_{i 1} \mathbf{E}_{i 1}^{\top}+\mathbf{R}_{i 1}\right) \mathbf{P}_{i 1}^{*} \quad \mathrm{e} \\
\mathbf{D}_{i 9}=\sum_{k=1}^{Q} \frac{\omega_{k}}{\sqrt{\pi}} A_{i} \mathbf{P}_{i 1}\left(\mathbf{E}_{i 1} \mathbf{E}_{i 3}^{\top}+\mathbf{R}_{i 3}\right) \mathbf{P}_{i 1}^{*},
\end{gathered}
$$

respectivamente, com $\mathbf{R}_{i 3}=\operatorname{diag}\left\{r_{i 13}, \ldots, r_{i m_{i}}\right\}$ uma matriz $m_{i} \times m_{i}$ com elementos $r_{i j 3}=-v_{i j}$. 
$\mathbf{D}_{i 10}$ e $D_{i 11}$ são dados respectivamente por

$$
\begin{gathered}
\mathbf{D}_{i 10}=\sum_{k=1}^{Q} \frac{\omega_{k}}{\sqrt{\pi}} A_{i}^{*} E_{4} \mathbf{P}_{i 1} \mathbf{E}_{i 1} \mathbf{P}_{i 1}^{* \top} \quad \mathrm{e} \\
D_{i 11}=\sum_{k=1}^{Q} \frac{\omega_{k}}{\sqrt{\pi}} A_{i} \mathbf{P}_{i 1}^{* \top}\left(\mathbf{E}_{i 2} \mathbf{E}_{i 2}^{\top}+\mathbf{R}_{i 4}\right) \mathbf{P}_{i 1}^{*},
\end{gathered}
$$

$\operatorname{com} \mathbf{R}_{i 4}=\operatorname{diag}\left\{r_{i 14}, \ldots, r_{i m_{i}}\right\}$ uma matriz $m_{i} \times m_{i}$ de elementos $r_{i j 4}=1-3 z_{i j}^{2}-\left(\alpha_{e}-\right.$ 1) $\left(-2 z_{i j} v_{i j}+z_{i j}^{2} v_{i j}+z_{i j}^{2} v_{i j}^{2}\right)$. As quantidades $D_{i 12}$ e $D_{i 13}$ são dadas por

$$
\begin{gathered}
D_{i 12}=\sum_{k=1}^{Q} \frac{\sqrt{2} u_{k} \omega_{k}}{\sqrt{\pi}} A_{i} \mathbf{P}_{i 1}^{* \top}\left(\mathbf{E}_{i 2} \mathbf{E}_{i 1}^{\top}+\mathbf{R}_{i 2}\right) \mathbf{P}_{i 1}^{*} \quad \mathrm{e} \\
D_{i 13}=\sum_{k=1}^{Q} \frac{\omega_{k}}{\sqrt{\pi}} A_{i} \mathbf{1}_{m_{i}}^{\top}\left(\mathbf{E}_{i 2} \mathbf{E}_{i 3}^{\top}+\mathbf{R}_{i 5}\right) \mathbf{P}_{i 1}^{*}
\end{gathered}
$$

respectivamente, com $\mathbf{R}_{i 5}=\operatorname{diag}\left\{r_{i 15}, \ldots, r_{i m_{i}} 5\right\}$ uma matriz $m_{i} \times m_{i}$ de elementos $r_{i j 5}=$ $-z_{i j} v_{i j}$. As quantidades $D_{i 14}, D_{i 15}$ e $D_{i 16}$ são dadas por

$$
\begin{gathered}
D_{i 14}=\sum_{k=1}^{Q} \frac{\omega_{k}}{\sqrt{\pi}} A_{i} E_{4} \mathbf{P}_{i 1}^{* \top} \mathbf{E}_{i 2}, \\
D_{i 15}=\sum_{k=1}^{Q} \frac{2 u_{k}^{2} \omega_{k}}{\sqrt{\pi}} A_{i} \mathbf{P}_{i 1}^{* \top}\left(\mathbf{E}_{i 1} \mathbf{E}_{i 1}^{\top}+\mathbf{R}_{i 1}\right) \mathbf{P}_{i 1}^{*} \quad \mathrm{e} \\
D_{i 16}=\sum_{k=1}^{Q} \frac{\sqrt{2} u_{k} \omega_{k}}{\sqrt{\pi}} A_{i}^{*} \mathbf{1}_{n_{i}}^{\top}\left(\mathbf{E}_{i 1} \mathbf{E}_{i 3}^{\top}+\mathbf{R}_{i 6}\right) \mathbf{P}_{i 1}^{*},
\end{gathered}
$$

$\operatorname{com} \mathbf{R}_{i 6}=\operatorname{diag}\left\{r_{i 16}, \ldots, r_{i m_{i}} 6\right\}$ uma matriz $m_{i} \times m_{i}$ de elementos $r_{i j 6}=-v_{i j}$. As quantidades $D_{i 17}$ e $D_{i 18}$ são dadas por

$$
\begin{gathered}
D_{i 17}=\sum_{k=1}^{Q} \frac{\sqrt{2} u_{k} \omega_{k}}{\sqrt{\pi}} A_{i} E_{4} \mathbf{P}_{i 1}^{* \top} \mathbf{E}_{i 1} \\
D_{i 18}=\sum_{k=1}^{Q} \frac{\omega_{k}}{\sqrt{\pi}} A_{i} \mathbf{1}_{m_{i}}^{\top}\left(\mathbf{E}_{i 3} \mathbf{E}_{i 3}^{\top}+\mathbf{R}_{i 7}\right) \mathbf{1}_{m_{i}},
\end{gathered}
$$

$\operatorname{com} \mathbf{R}_{i 7}=\operatorname{diag}\left\{r_{i 17}, \ldots, r_{i m_{i}} 7\right\}$ uma matriz $m_{i} \times m_{i}$ de elementos $r_{i j 7}=-1 / \alpha_{e}^{2}$. Finalmente, nós definimos

$$
\begin{aligned}
D_{i 19} & =\sum_{k=1}^{Q} \frac{\omega_{k}}{\sqrt{\pi}} A_{i} E_{4} \mathbf{1}_{m_{i}}^{\top} \mathbf{E}_{i 3} \quad \mathrm{e} \\
D_{i 20} & =\sum_{k=1}^{Q} \frac{\omega_{k}}{\sqrt{\pi}} A_{i}\left(E_{4}^{2}-\frac{1}{\alpha_{g}^{2}}\right) .
\end{aligned}
$$




\section{B.5.3 Função Escore para o PNLMM}

Considerando a fdp da distribuição normal-potência (2.13) e considerando o modelo de regressão linear misto normal-potência dado em (4.17), temos que

$$
f_{Y \mid \boldsymbol{\gamma}}\left(y_{i j} \mid \mathbf{u}_{k}, \boldsymbol{\eta}_{g} ; \boldsymbol{\beta}, \eta_{e}, \alpha_{e}\right)=\frac{\alpha_{e}}{\eta_{e}} \phi\left(z_{i j}\right)\left\{\Phi\left(z_{i j}\right)\right\}^{\alpha_{e}-1}
$$

em que $z_{i j}=\left(y_{i j}-\mathbf{x}_{i j}^{\top} \boldsymbol{\beta}-\sqrt{2} \mathbf{w}_{i j}^{\top} \boldsymbol{\eta}_{g}^{\frac{1}{2}} \mathbf{u}_{k}\right) / \eta_{e}, \mathbf{u}_{k}=\left(u_{k_{1}}, \ldots, u_{k_{q}}\right)^{\top}$, e assim, o logaritmo da função de verossimilhança aproximada (4.23) pode ser expresso como

$$
\ell(\boldsymbol{\theta} ; \mathbf{Y}) \approx \sum_{i=1}^{n} \log B_{i}
$$

em que

$$
B_{i}=\sum_{k_{1}=1}^{Q_{1}} \cdots \sum_{k_{q}=1}^{Q_{q}} \frac{\omega_{k_{1}} \cdots \omega_{k_{q}}}{\pi^{q / 2}} A_{i}
$$

com

$$
\begin{aligned}
A_{i}=\exp & \left\{m_{i} \log \left(\frac{\alpha_{e}}{\eta_{e}}\right)+\sum_{j=1}^{m_{i}} \log \phi\left(z_{i j}\right)+\left(\alpha_{e}-1\right) \sum_{j=1}^{m_{i}} \log \Phi\left(z_{i j}\right)\right. \\
& +\sum_{l=1}^{q} \log \left(\alpha_{l}\right)+\sum_{l=1}^{q-1} \log \left(1+\frac{l}{\alpha}\right)-\sum_{l=1}^{q}\left(\frac{\alpha_{l}}{\alpha}+1\right) \log \Phi\left(\sqrt{2} u_{k_{l}}\right) \\
& \left.-(\alpha+q) \log \left\{\sum_{l=1}^{q}\left[\Phi\left(\sqrt{2} u_{k_{l}}\right)\right]^{-\alpha_{l} / \alpha}-(q-1)\right\}\right\}
\end{aligned}
$$

sendo $u_{k_{l}}$ e $\omega_{k_{l}}$ os pontos e os pesos da quadratura de Gauss-Hermite multidemensional para $l=1, \ldots, q$, respectivamente. A função escore é obtida diferenciando o logaritmo da função de verossimilhança (B.5) em relação a cada um dos parâmetros, e fica dada por

$$
\boldsymbol{U}(\boldsymbol{\theta})=\left(\boldsymbol{U}_{\boldsymbol{\beta}}^{\top}(\boldsymbol{\theta}), \boldsymbol{U}_{\boldsymbol{\eta}_{g}}^{\top}(\boldsymbol{\theta}), \boldsymbol{U}_{\boldsymbol{\alpha}_{g}}^{\top}(\boldsymbol{\theta}), U_{\eta_{e}}(\boldsymbol{\theta}), U_{\alpha_{e}}(\boldsymbol{\theta}),\right)^{\top},
$$

em que $\boldsymbol{U}_{\boldsymbol{\beta}}(\boldsymbol{\theta})=\left(U_{\beta_{1}}(\boldsymbol{\theta}), \ldots, U_{\beta_{p}}(\boldsymbol{\theta})\right)^{\top}, \boldsymbol{U}_{\boldsymbol{\eta}_{g}}(\boldsymbol{\theta})=\left(U_{\eta_{1}}(\boldsymbol{\theta}), \ldots, U_{\eta_{q}}(\boldsymbol{\theta})\right)^{\top}$, e $\boldsymbol{U}_{\boldsymbol{\alpha}_{g}}(\boldsymbol{\theta})=\left(U_{\alpha_{1}}(\boldsymbol{\theta}), \ldots, U_{\alpha_{q}}(\boldsymbol{\theta}), U_{\alpha}(\boldsymbol{\theta})\right)^{\top}, \mathrm{com}$

$$
\begin{aligned}
U_{\beta_{l}}(\boldsymbol{\theta})= & \sum_{i=1}^{n} \frac{1}{B_{i}} \sum_{k_{1}=1}^{Q_{1}} \cdots \sum_{k_{q}=1}^{Q_{q}} \frac{\omega_{k_{1}} \cdots \omega_{k_{q}}}{\pi^{q / 2}} A_{i} \sum_{j=1}^{m_{i}}\left\{z_{i j}-\left(\alpha_{e}-1\right) \frac{\phi\left(z_{i j}\right)}{\Phi\left(z_{i j}\right)}\right\} \frac{x_{i j l}}{\eta_{e}} \\
& \operatorname{para} l=1, \ldots, p \\
U_{\eta_{r}}(\boldsymbol{\theta})= & \sum_{i=1}^{n} \frac{1}{B_{i}} \sum_{k_{1}=1}^{Q_{1}} \cdots \sum_{k_{q}=1}^{Q_{q}} \frac{\omega_{k_{1}} \cdots \omega_{k_{q}}}{\pi^{q / 2}} A_{i} \sum_{j=1}^{m_{i}}\left\{z_{i j}-\left(\alpha_{e}-1\right) \frac{\phi\left(z_{i j}\right)}{\Phi\left(z_{i j}\right)}\right\} \frac{\sqrt{2} w_{i j r} u_{k_{r}}}{\eta_{e}} \\
& \operatorname{para} r=1, \ldots, q
\end{aligned}
$$




$$
\begin{aligned}
U_{\alpha_{r}}(\boldsymbol{\theta})= & \sum_{i=1}^{n} \frac{1}{B_{i}} \sum_{k_{1}=1}^{Q_{1}} \cdots \sum_{k_{q}=1}^{Q_{q}} \frac{\omega_{k_{1}} \cdots \omega_{k_{q}}}{\pi^{q / 2}} A_{i} \\
& \times\left\{\frac{1}{\alpha_{r}}-\frac{1}{\alpha} \log \Phi\left(\sqrt{2} u_{k_{r}}\right)\left(1-\frac{(\alpha+q)\left[\Phi\left(\sqrt{2} u_{k_{r}}\right)\right]^{-\alpha_{r} / \alpha}}{\sum_{l=1}^{q}\left[\Phi\left(\sqrt{2} u_{k_{l}}\right)\right]^{-\alpha_{l} / \alpha}-(q-1)}\right)\right\} \\
& \operatorname{para} r=1, \ldots, q ; \\
U_{\alpha}(\boldsymbol{\theta})= & \sum_{i=1}^{n} \frac{1}{B_{i}} \sum_{k_{1}=1}^{Q_{1}} \cdots \sum_{k_{q}=1}^{Q_{q}} \frac{\omega_{k_{1}} \cdots \omega_{k_{q}}}{\pi^{q / 2}} A_{i} \\
& \times\left\{-\sum_{l=1}^{q-1} \frac{l}{\alpha(\alpha+l)}+\frac{1}{\alpha^{2}} \sum_{l=1}^{q} \alpha_{l} \log \Phi\left(\sqrt{2} u_{k_{l}}\right)\right. \\
& -\log \left\{\sum_{l=1}^{q}\left[\Phi\left(\sqrt{2} u_{k_{l}}\right)\right]^{-\alpha_{l} / \alpha}-(q-1)\right\} \\
& \left.+\frac{(\alpha+q) \sum_{l=1}^{q} \alpha_{l}\left[\Phi\left(\sqrt{2} u_{k_{l}}\right)\right]^{-\alpha_{l} / \alpha} \log \Phi\left(\sqrt{2} u_{k_{l}}\right)}{\alpha^{2}\left(\sum_{l=1}^{q}\left[\Phi\left(\sqrt{2} u_{k_{l}}\right)\right]^{-\alpha_{l} / \alpha}-(q-1)\right)}\right\} \\
U_{\eta_{e}}(\boldsymbol{\theta})= & \sum_{i=1}^{n} \frac{1}{B_{i}} \sum_{k_{1}=1}^{Q_{1}} \cdots \sum_{k_{q}=1}^{Q_{q}} \frac{\omega_{k_{1}} \cdots \omega_{k_{q}}}{\pi^{q / 2}} A_{i} \sum_{j=1}^{m_{i}}\left\{-1+z_{i j}^{2}-\left(\alpha_{e}-1\right) \frac{\phi\left(z_{i j}\right)}{\Phi\left(z_{i j}\right)}\right\} \frac{1}{\eta_{e}} ; \\
U_{\alpha_{e}}(\boldsymbol{\theta})= & \sum_{i=1}^{n} \frac{1}{B_{i}} \sum_{k_{1}=1}^{Q_{1}} \ldots \sum_{k_{q}=1}^{Q_{q}} \frac{\omega_{k_{1}} \cdots \omega_{k_{q}}}{\pi^{q / 2}} A_{i} \sum_{j=1}^{m_{i}}\left\{\frac{1}{\alpha_{e}}+\log \Phi\left(z_{i j}\right)\right\} ;
\end{aligned}
$$

\section{B.6 Função Escore e Matriz de Informação Observada para o Modelo PNDC-RE}

Apresentamos nesta seção as expressões para elementos da função escore e a matriz de informação observada do modelo PNDC-RE. O logaritmo da função de verossimilhança $\ell\left(\boldsymbol{\theta} ; \mathbf{Y}^{o}\right)$ dado em (5.23) pode ser expresso como

$$
\ell\left(\boldsymbol{\theta} ; \mathbf{Y}^{o}\right)=\sum_{i=1}^{n} \log B_{i}
$$

em que

$$
B_{i}=\sum_{k=1}^{Q} \frac{\omega_{k}}{\sqrt{\pi}} A_{i}
$$

com

$$
\begin{aligned}
A_{i}=\exp & \left\{\sum_{j=1}^{m_{i}} d_{0 i j} \alpha_{e} \log \Phi\left(z_{0 i j}\right)+d_{2 i j} \log \left(1-\left\{\Phi\left(z_{2 i j}\right)\right\}^{\alpha_{e}}\right)\right. \\
+ & \left(1-d_{0 i j}-d_{2 i j}\right)\left(\log \left(\alpha_{e} / \eta_{e}\right)+\log \phi\left(z_{i j}\right)+\left(\alpha_{e}-1\right) \log \Phi\left(z_{i j}\right)\right) \\
& \left.+\log \alpha_{g}+\left(\alpha_{g}-1\right) \log \Phi\left(\sqrt{2} u_{k}\right)\right\}
\end{aligned}
$$


sendo $z_{0 i j}=\left(c_{0 i j}-\mathbf{x}_{i j}^{\top} \boldsymbol{\beta}-\sqrt{2} \eta_{g} u_{k}\right) / \eta_{e}, z_{2 i j}=\left(c_{2 i j}-\mathbf{x}_{i j}^{\top} \boldsymbol{\beta}-\sqrt{2} \eta_{g} u_{k}\right) / \eta_{e}$ e $z_{i j}=\left(y_{i j}-\mathbf{x}_{i j}^{\top} \boldsymbol{\beta}-\right.$ $\left.\sqrt{2} \eta_{g} u_{k}\right) / \eta_{e}$

\section{B.6.1 Função Escore}

Diferenciando o logaritmo da função de verossimilhança (B.6) em relação a cada um dos parâmetros obtemos os componentes do vetor de escore $\boldsymbol{U}(\boldsymbol{\theta})$ dado em (5.24) a seguir:

$$
\begin{aligned}
\boldsymbol{U}_{\boldsymbol{\beta}}(\boldsymbol{\theta}) & =\sum_{i=1}^{n} \frac{1}{B_{i}} \sum_{k=1}^{Q} \frac{\omega_{k}}{\sqrt{\pi}} A_{i}\left(-\alpha_{e} \mathbf{X}_{0 i}^{\top} \boldsymbol{\Lambda}_{i} \mathbf{W}_{0 i}+\mathbf{X}_{1 i}^{\top} \boldsymbol{\Lambda}_{i} \mathbf{Q}_{1 i}+\mathbf{X}_{2 i}^{\top} \boldsymbol{\Lambda}_{i} \mathbf{H}_{2 i}\right), \\
U_{\eta_{e}}(\boldsymbol{\theta}) & =\sum_{i=1}^{n} \frac{1}{B_{i}} \sum_{k=1}^{Q} \frac{\omega_{k}}{\sqrt{\pi}} A_{i}\left(-\alpha_{e} \mathbf{Z}_{0 i}^{\top} \mathbf{\Lambda}_{i} \mathbf{W}_{0 i}+\boldsymbol{\Lambda}_{i}^{* \top} \mathbf{Q}_{2 i}+\mathbf{Z}_{2 i}^{\top} \boldsymbol{\Lambda}_{i} \mathbf{H}_{2 i}\right), \\
U_{\eta_{g}}(\boldsymbol{\theta}) & =\sum_{i=1}^{n} \frac{1}{B_{i}} \sum_{k=1}^{Q} \frac{\sqrt{2} \omega_{k} u_{k}}{\sqrt{\pi}} A_{i} \mathbf{\Lambda}_{i}^{* \top}\left(-\alpha_{e} \mathbf{W}_{0 i}+\mathbf{Q}_{1 i}+\mathbf{H}_{2 i}\right), \\
U_{\alpha_{e}}(\boldsymbol{\theta}) & =\sum_{i=1}^{n} \frac{1}{B_{i}} \sum_{k=1}^{Q} \frac{\omega_{k}}{\sqrt{\pi}} A_{i}\left(\mathbf{1}_{m_{i}}^{\top} \mathbf{P}_{0 i}+\mathbf{1}_{n_{i}}^{\top} \mathbf{Q}_{3 i}-\frac{1}{\alpha_{e}} \mathbf{P}_{2 i}^{\top} \mathbf{H}_{2 i}\right), \\
U_{\alpha_{g}}(\boldsymbol{\theta}) & =\sum_{i=1}^{n} \frac{1}{B_{i}} \sum_{k=1}^{Q} \frac{\omega_{k}}{\sqrt{\pi}} A_{i}\left(\frac{1}{\alpha_{g}}+\log \Phi\left(\sqrt{2} u_{k}\right)\right),
\end{aligned}
$$

em que $\mathbf{X}_{0 i}, \mathbf{X}_{1 i}$ e $\mathbf{X}_{2 i}$ são matrizes de dimensão $m_{i} \times 1$ associadas aos valores $Y_{i j}^{o}=c_{0 i j}$, $Y_{i j}^{o}=Y_{i j}$ e $Y_{i j}=c_{2 i j}$ respectivamente; $\mathbf{Z}_{0 i}=\left(z_{0 i 1}, \ldots, z_{0 i m_{i}}\right)^{\top}$ e $\mathbf{Z}_{2 i}=\left(z_{2 i 1}, \ldots, z_{2 i m_{i}}\right)^{\top}$ são vetores de dimensaõ $m_{i} \times 1 ; \boldsymbol{\Lambda}_{i}=\operatorname{diag}\left\{1 / \eta_{e}, \ldots, 1 / \eta_{e}\right\}$ é uma matriz de dimensão $m_{i} \times 1$, e $\boldsymbol{\Lambda}_{i}^{*}=\left(1 / \eta_{e}, \ldots, 1 / \eta_{e}\right)^{\top}$ é um vetor de dimensão $m_{i} \times 1$. As quantidades, $\mathbf{Q}_{1 i}, \mathbf{Q}_{2 i}$ e $\mathbf{Q}_{3 i}$ são vetores de dimensão $m_{i} \times 1$ com componentes dados respectivamente por $q_{1 i j}=z_{i j}-\left(\alpha_{e}-1\right) w_{i j}$, $q_{2 i j}=-1+z_{i j}^{2}-\left(\alpha_{e}-1\right) z_{i j} w_{i j}$ e $q_{3 i j}=\frac{1}{\alpha_{e}}+\log \Phi\left(z_{i j}\right)$ com $w_{i j}=\phi\left(z_{i j}\right) / \Phi\left(z_{i j}\right) . \mathbf{W}_{0 i}=$ $\left(w_{0 i 1}, \ldots, w_{0 i m_{i}}\right)^{\top} \operatorname{com} w_{0 i j}=\phi\left(z_{0 i j}\right) / \Phi\left(z_{0 i j}\right)$ e $\mathbf{H}_{2 i}=\left(h_{2 i 1}, \ldots, h_{2 i m_{i}}\right)^{\top} ; \mathbf{P}_{0 i}$, e $\mathbf{P}_{2 i}$ são vetores $m_{i} \times 1 \operatorname{com} p_{0 i j}=\log \Phi\left(z_{0 i j}\right)$ e $p_{2 i j}=w_{2 i j}^{-1} \log \Phi\left(z_{2 i j}\right) \operatorname{com} w_{2 i j}=\phi\left(z_{2 i j}\right) / \Phi\left(z_{2 i j}\right)$. Finalmente $r_{0 i j}=\alpha_{e} \phi\left(z_{0 i j}\right) / \Phi\left(z_{0 i j}\right)$ e $h_{2 i j}=\left(\alpha_{e} \phi\left(z_{2 i j}\right)\left[\Phi\left(z_{2 i j}\right)\right]^{\alpha_{e}-1}\right) /\left(1-\left[\Phi\left(z_{2 i j}\right)\right]^{\alpha_{e}}\right)$ são as funções de risco e risco inverso da distribuição normal-potência respectivamente.

\section{B.6.2 Matriz de Informação Observada}

Os elementos da matriz de informação observada $\boldsymbol{j}_{\boldsymbol{\theta}_{j} \boldsymbol{\theta}_{k}}$ para o modelo PNDC-RE dada em são obtidos calculado a segunda derivada da função de log-verossimilhança (B.6) em relação a cada um dos parâmetros, isto é, usando a expressão

$$
\begin{aligned}
\boldsymbol{j}_{\boldsymbol{\theta}_{j} \boldsymbol{\theta}_{k}} & =-\sum_{i=1}^{n} \frac{\partial \boldsymbol{U}_{k}(\boldsymbol{\theta})}{\partial \boldsymbol{\theta}_{j}} \\
& =-\sum_{i=1}^{n} \frac{\partial}{\partial \boldsymbol{\theta}_{j}}\left(\frac{1}{B_{i}} \frac{\partial B_{i}}{\partial \boldsymbol{\theta}_{k}}\right) \\
& =-\sum_{i=1}^{n}\left(\frac{1}{B_{i}} \frac{\partial^{2} B_{i}}{\partial \boldsymbol{\theta}_{j} \partial \boldsymbol{\theta}_{k}}-\frac{1}{B_{i}^{2}} \frac{\partial B_{i}}{\partial \boldsymbol{\theta}_{j}} \frac{\partial B_{i}}{\partial \boldsymbol{\theta}_{k}}\right)
\end{aligned}
$$


e são dados por

$$
\begin{aligned}
& j_{\boldsymbol{\beta}_{l} \boldsymbol{\beta}_{l^{\prime}}}=-\sum_{i=1}^{n}\left\{\frac { 1 } { B _ { i } } \sum _ { k = 1 } ^ { Q } \frac { \omega _ { k } } { \sqrt { \pi } } A _ { i } \left[\left(\sum_{j=1}^{m_{i}}\left[-\frac{\alpha_{e} w_{0 i j} x_{i j l}}{\eta_{e}}\right]^{d_{0 i j}}\left[\frac{\left(z_{i j}-\left(\alpha_{e}-1\right) w_{i j}\right) x_{i j l}}{\eta_{e}}\right]^{1-d_{0 i j}-d_{2 i j}}\left[\frac{h_{2 i j} x_{i j l}}{\eta_{e}}\right]^{d_{2 i j}}\right)\right.\right. \\
& \left(\sum_{j=1}^{m_{i}}\left[-\frac{\alpha_{e} w_{0 i j} x_{i j l^{\prime}}}{\eta_{e}}\right]^{d_{0 i j}}\left[\frac{\left(z_{i j}-\left(\alpha_{e}-1\right) w_{i j}\right) x_{i j l^{\prime}}}{\eta_{e}}\right]^{1-d_{0 i j}-d_{2 i j}}\left[\frac{h_{2 i j} x_{i j l^{\prime}}}{\eta_{e}}\right]^{d_{2 i j}}\right) \\
& +\sum_{j=1}^{m_{i}}\left[-\frac{\alpha_{e}\left(z_{0 i j}+w_{0 i j}\right) w_{0 i j} x_{i j l} x_{i j l^{\prime}}}{\eta_{e}^{2}}\right]^{d_{0 i j}}\left[-\frac{\left(1+\left(\alpha_{e}-1\right)\left(z_{i j}+w_{i j}\right) w_{i j}\right) x_{i j l} x_{i j l^{\prime}}}{\eta_{e}^{2}}\right]^{1-d_{0 i j}-d_{2 i j}} \\
& \left.\left[\frac{\left(z_{2 i j}-\left(\alpha_{e}-1\right) w_{2 i j}-h_{2 i j}\right) h_{2 i j} x_{i j l} x_{i j l^{\prime}}}{\eta_{e}^{2}}\right]^{d_{2 i j}}\right] \\
& -\frac{1}{B_{i}^{2}}\left(\sum_{k=1}^{Q} \frac{\omega_{k}}{\sqrt{\pi}} A_{i} \sum_{j=1}^{m_{i}}\left[-\frac{\alpha_{e} w_{0 i j} x_{i j l}}{\eta_{e}}\right]^{d_{0 i j}}\left[\frac{\left(z_{i j}-\left(\alpha_{e}-1\right) w_{i j}\right) x_{i j l}}{\eta_{e}}\right]^{1-d_{0 i j}-d_{2 i j}}\left[\frac{h_{2 i j} x_{i j l}}{\eta_{e}}\right]^{d_{2 i j}}\right) \\
& \left.\left(\sum_{k=1}^{Q} \frac{\omega_{k}}{\sqrt{\pi}} A_{i} \sum_{j=1}^{m_{i}}\left[-\frac{\alpha_{e} w_{0 i j} x_{i j l^{\prime}}}{\eta_{\varepsilon}}\right]^{d_{0 i j}}\left[\frac{\left(z_{i j}-\left(\alpha_{e}-1\right) w_{i j}\right) x_{i j l^{\prime}}}{\eta_{e}}\right]^{1-d_{0 i j}-d_{2 i j}}\left[\frac{h_{2 i j} x_{i j l^{\prime}}}{\eta_{e}}\right]^{d_{2 i j}}\right)\right\} \\
& j_{\boldsymbol{\beta}_{l} \eta_{e}}=-\sum_{i=1}^{n}\left\{\frac { 1 } { B _ { i } } \sum _ { k = 1 } ^ { Q } \frac { \omega _ { k } } { \sqrt { \pi } } A _ { i } \left[\left(\sum_{j=1}^{m_{i}}\left[-\frac{\alpha_{e} w_{0 i j} x_{i j l}}{\eta_{e}}\right]^{d_{0 i j}}\left[\frac{\left(z_{i j}-\left(\alpha_{e}-1\right) w_{i j}\right) x_{i j l}}{\eta_{e}}\right]^{1-d_{0 i j}-d_{2 i j}}\left[\frac{h_{2 i j} x_{i j l}}{\eta_{e}}\right]^{d_{2 i j}}\right)\right.\right. \\
& \left(\sum_{j=1}^{n_{i}}\left[-\frac{\alpha_{e} z_{0 i j} w_{0 i j}}{\eta_{e}}\right]^{d_{0 i j}}\left[\frac{-1+z_{i j}^{2}-\left(\alpha_{e}-1\right) z_{i j} w_{i j}}{\eta_{e}}\right]^{1-d_{0 i j}-d_{2 i j}}\left[\frac{h_{2 i j}}{\eta_{e}}\right]^{d_{2 i j}}\right) \\
& +\sum_{j=1}^{n_{i}}\left[-\frac{\alpha_{e}\left(z_{0 i j}^{2}-1+w_{0 i j} z_{0 i j}\right) w_{0 i j} x_{i j l}}{\eta_{e}^{2}}\right]^{d_{0 i j}}\left[\frac{\left(-2 z_{i j}-\left(\alpha_{e}-1\right)\left(z_{i j}^{2} w_{i j}-w_{i j}+z_{i j} w_{i j}^{2}\right)\right) x_{i j l}}{\eta_{e}^{2}}\right]^{1-d_{0 i j}-d_{2 i j}} \\
& \left.\left[\frac{\left(z_{2 i j}^{2}-\left(\alpha_{e}-1\right) z_{2 i j} w_{2 i j}-1-z_{2 i j} h_{2 i j}\right) h_{2 i j} x_{i j l}}{\eta_{e}^{2}}\right]^{d_{2 i j}}\right] \\
& -\frac{1}{B_{i}^{2}}\left(\sum_{k=1}^{Q} \frac{\omega_{k}}{\sqrt{\pi}} A_{i} \sum_{j=1}^{m_{i}}\left[-\frac{\alpha_{e} w_{0 i j} x_{i j l}}{\eta_{e}}\right]^{d_{0 i j}}\left[\frac{\left(z_{i j}-\left(\alpha_{e}-1\right) w_{i j}\right) x_{i j l}}{\eta_{e}}\right]^{1-d_{0 i j}-d_{2 i j}}\left[\frac{h_{2 i j} x_{i j l}}{\eta_{e}}\right]^{d_{2 i j}}\right) \\
& \left.\left(\sum_{k=1}^{Q} \frac{\omega_{k}}{\sqrt{\pi}} A_{i} \sum_{j=1}^{m_{i}}\left[-\frac{\alpha_{e} z_{0 i j} w_{0 i j}}{\eta_{e}}\right]^{d_{0 i j}}\left[\frac{-1+z_{i j}^{2}-\left(\alpha_{e}-1\right) z_{i j} w_{i j}}{\eta_{e}}\right]^{1-d_{0 i j}-d_{2 i j}}\left[\frac{h_{2 i j}}{\eta_{e}}\right]^{d_{2 i j}}\right)\right\}
\end{aligned}
$$




$$
\begin{aligned}
& j_{\boldsymbol{\beta}_{l} \eta_{g}}=-\sum_{i=1}^{n}\left\{\frac { 1 } { B _ { i } } \sum _ { k = 1 } ^ { Q } \frac { \omega _ { k } } { \sqrt { \pi } } A _ { i } \left[\left(\sum_{j=1}^{m_{i}}\left[-\frac{\alpha_{e} w_{0 i j} x_{i j l}}{\eta_{e}}\right]^{d_{0 i j}}\left[\frac{\left(z_{i j}-\left(\alpha_{e}-1\right) w_{i j}\right) x_{i j l}}{\eta_{e}}\right]^{1-d_{0 i j}-d_{2 i j}}\left[\frac{h_{2 i j} x_{i j l}}{\eta_{e}}\right]^{d_{2 i j}}\right)\right.\right. \\
& \left(\sum_{j=1}^{m_{i}}\left[-\frac{\alpha_{e} w_{0 i j} \sqrt{2} u_{k}}{\eta_{e}}\right]^{d_{0 i j}}\left[\frac{\left(z_{i j}-\left(\alpha_{e}-1\right) w_{i j}\right) \sqrt{2} u_{k}}{\eta_{e}}\right]^{1-d_{0 i j}-d_{2 i j}}\left[\frac{h_{2 i j} \sqrt{2} u_{k}}{\eta_{e}}\right]^{d_{2 i j}}\right) \\
& +\sum_{j=1}^{m_{i}}\left[-\frac{\alpha_{e}\left(z_{0 i j}+w_{0 i j}\right) w_{0 i j} x_{i j l} \sqrt{2} u_{k}}{\eta_{e}^{2}}\right]^{d_{0 i j}}\left[-\frac{\left(1+\left(\alpha_{e}-1\right)\left(z_{i j}+w_{i j}\right) w_{i j}\right) x_{i j l} \sqrt{2} u_{k}}{\eta_{e}^{2}}\right]^{1-d_{0 i j}-d_{2 i j}} \\
& \left.\left[\frac{\left(z_{2 i j}-\left(\alpha_{e}-1\right) w_{2 i j}-h_{2 i j}\right) h_{2 i j} x_{i j l} \sqrt{2} u_{k}}{\eta_{e}^{2}}\right]^{d_{2 i j}}\right] \\
& -\frac{1}{B_{i}^{2}}\left(\sum_{k=1}^{Q} \frac{\omega_{k}}{\sqrt{\pi}} A_{i} \sum_{j=1}^{m_{i}}\left[-\frac{\alpha_{e} w_{0 i j} x_{i j l}}{\eta_{e}}\right]^{d_{0 i j}}\left[\frac{\left(z_{i j}-\left(\alpha_{e}-1\right) w_{i j}\right) x_{i j l}}{\eta_{e}}\right]^{1-d_{0 i j}-d_{2 i j}}\left[\frac{h_{2 i j} x_{i j l}}{\eta_{e}}\right]^{d_{2 i j}}\right) \\
& \left.\left(\sum_{k=1}^{Q} \frac{\omega_{k}}{\sqrt{\pi}} A_{i} \sum_{j=1}^{m_{i}}\left[-\frac{\alpha_{e} w_{0 i j} \sqrt{2} u_{k}}{\eta_{e}}\right]^{d_{0 i j}}\left[\frac{\left(z_{i j}-\left(\alpha_{e}-1\right) w_{i j}\right) \sqrt{2} u_{k}}{\eta_{e}}\right]^{1-d_{0 i j}-d_{2 i j}}\left[\frac{h_{2 i j} \sqrt{2} u_{k}}{\eta_{e}}\right]^{d_{2 i j}}\right)\right\} \\
& j_{\boldsymbol{\beta}_{l} \alpha_{e}}=-\sum_{i=1}^{n}\left\{\frac { 1 } { B _ { i } } \sum _ { k = 1 } ^ { Q } \frac { \omega _ { k } } { \sqrt { \pi } } A _ { i } \left[\left(\sum_{j=1}^{m_{i}}\left[-\frac{\alpha_{e} w_{0 i j} x_{i j l}}{\eta_{e}}\right]^{d_{0 i j}}\left[\frac{\left(z_{i j}-\left(\alpha_{e}-1\right) w_{i j}\right) x_{i j l} l}{\eta_{e}}\right]^{1-d_{0 i j}-d_{2 i j}}\left[\frac{h_{2 i j} x_{i j l}}{\eta_{e}}\right]^{d_{2 i j}}\right)\right.\right. \\
& \left(\sum_{j=1}^{m_{i}}\left[\log \Phi\left(z_{0 i j}\right)\right]^{d_{0 i j}}\left[\frac{1}{\alpha_{e}}+\log \Phi\left(z_{i j}\right)\right]^{1-d_{0 i j}-d_{2 i j}}\left[-\frac{h_{2 i j} \log \Phi\left(z_{2 i j}\right)}{\alpha_{e} w_{2 i j}}\right]^{d_{2 i j}}\right) \\
& +\sum_{j=1}^{m_{i}}\left[-\frac{w_{0 i j} x_{i j l}}{\eta_{e}}\right]^{d_{0 i j}}\left[-\frac{w_{i j} x_{i j l}}{\eta_{e}}\right]^{1-d_{0 i j}-d_{2 i j}} \\
& \left.\left[\frac{\left(h_{2 i j}\left(\alpha_{e}^{-1}+\log \Phi\left(z_{2 i j}\right)\right)+\alpha_{e}^{-1} w_{2 i j}^{-1} h_{2 i j} \log \Phi\left(z_{2 i j}\right)\right) x_{i j l}}{\eta_{e}}\right]^{d_{2 i j}}\right] \\
& -\frac{1}{B_{i}^{2}}\left(\sum_{k=1}^{Q} \frac{\omega_{k}}{\sqrt{\pi}} A_{i} \sum_{j=1}^{m_{i}}\left[-\frac{\alpha_{e} w_{0 i j} x_{i j l}}{\eta_{e}}\right]^{d_{0 i j}}\left[\frac{\left(z_{i j}-\left(\alpha_{e}-1\right) w_{i j}\right) x_{i j l}}{\eta_{e}}\right]^{1-d_{0 i j}-d_{2 i j}}\left[\frac{h_{2 i j} x_{i j l}}{\eta_{e}}\right]^{d_{2 i j}}\right) \\
& \left.\left(\sum_{k=1}^{Q} \frac{\omega_{k}}{\sqrt{\pi}} A_{i} \sum_{j=1}^{m_{i}}\left[\log \Phi\left(z_{0 i j}\right)\right]^{d_{0 i j}}\left[\frac{1}{\alpha_{e}}+\log \Phi\left(z_{i j}\right)\right]^{1-d_{0 i j}-d_{2 i j}}\left[-\frac{h_{2 i j} \log \Phi\left(z_{2 i j}\right)}{\alpha_{e} w_{2 i j}}\right]^{d_{2 i j}}\right)\right\} \\
& j_{\boldsymbol{\beta}_{l} \alpha_{g}}=-\sum_{i=1}^{n}\left\{\frac { 1 } { B _ { i } } \sum _ { k = 1 } ^ { Q } \frac { \omega _ { k } } { \sqrt { \pi } } A _ { i } \left[\left(\sum_{j=1}^{m_{i}}\left[-\frac{\alpha_{e} w_{0 i j} x_{i j l}}{\eta_{e}}\right]^{d_{0 i j}}\left[\frac{\left(z_{i j}-\left(\alpha_{e}-1\right) w_{i j}\right) x_{i j l}}{\eta_{e}}\right]^{1-d_{0 i j}-d_{2 i j}}\left[\frac{h_{2 i j} x_{i j k}}{\eta_{e}}\right]^{d_{2 i j}}\right)\right.\right. \\
& \left.\left(\frac{1}{\alpha_{g}}+\log \Phi\left(\sqrt{2} u_{k}\right)\right)\right] \\
& -\frac{1}{B_{i}^{2}}\left(\sum_{k=1}^{Q} \frac{\omega_{k}}{\sqrt{\pi}} A_{i} \sum_{j=1}^{m_{i}}\left[-\frac{\alpha_{e} w_{0 i j} x_{i j l}}{\eta_{e}}\right]^{d_{0 i j}}\left[\frac{\left(z_{i j}-\left(\alpha_{e}-1\right) w_{i j}\right) x_{i j l}}{\eta_{e}}\right]^{1-d_{0 i j}-d_{2 i j}}\left[\frac{h_{2 i j} x_{i j l}}{\eta_{e}}\right]^{d_{2 i j}}\right) \\
& \left.\left(\sum_{k=1}^{Q} \frac{\omega_{k}}{\sqrt{\pi}} A_{i}\left[\frac{1}{\alpha_{g}}+\log \Phi\left(\sqrt{2} u_{k}\right)\right]\right)\right\}
\end{aligned}
$$




$$
\begin{aligned}
& j_{\eta_{e} \eta_{e}}=-\sum_{i=1}^{n}\left\{\frac { 1 } { B _ { i } } \sum _ { k = 1 } ^ { Q } \frac { \omega _ { k } } { \sqrt { \pi } } A _ { i } \left[\left(\sum_{j=1}^{m_{i}}\left[-\frac{\alpha_{e} z_{0 i j} w_{0 i j}}{\eta_{e}}\right]^{d_{0 i j}}\left[\frac{-1+z_{i j}^{2}-\left(\alpha_{e}-1\right) z_{i j} w_{i j}}{\eta_{e}}\right]^{1-d_{0 i j}-d_{2 i j}}\left[\frac{h_{2 i j}}{\eta_{e}}\right]^{d_{2 i j}}\right)^{2}\right.\right. \\
& +\sum_{j=1}^{m_{i}}\left[\frac{\alpha_{e}\left(2 z_{0 i j} w_{0 i j}-z_{0 i j}^{3} w_{0 i j}-z_{0 i j}^{2} w_{0 i j}^{2}\right)}{\eta_{e}^{2}}\right]^{d_{0 i j}}\left[\frac{1-3 z_{i j}^{2}-\left(\alpha_{e}-1\right)\left(z_{i j}^{3} w_{i j}+z_{i j}^{2} w_{i j}^{2}-2 z_{i j} w_{i j}\right)}{\eta_{e}^{2}}\right]^{1-d_{0 i j}-d_{2 i j}} \\
& \left.\left[\frac{\left(-1+z_{2 i j}^{2}-\left(\alpha_{e}-1\right) z_{2 i j} w_{2 i j}-z_{2 i j} h_{2 i j}\right) h_{2 i j}}{\eta_{e}^{2}}\right]^{d_{2 i j}}\right] \\
& \left.-\frac{1}{B_{i}^{2}}\left(\sum_{k=1}^{Q} \frac{\omega_{k}}{\sqrt{\pi}} A_{i} \sum_{j=1}^{m_{i}}\left[-\frac{\alpha_{e} z_{0 i j} w_{0 i j}}{\eta_{e}}\right]^{d_{0 i j}}\left[\frac{-1+z_{i j}^{2}-\left(\alpha_{e}-1\right) z_{i j} w_{i j}}{\eta_{e}}\right]^{1-d_{0 i j}-d_{2 i j}}\left[\frac{h_{2 i j}}{\eta_{e}}\right]^{d_{2 i j}}\right)^{2}\right\} \\
& j_{\eta_{e} \eta_{g}}=-\sum_{i=1}^{n}\left\{\frac { 1 } { B _ { i } } \sum _ { k = 1 } ^ { Q } \frac { \omega _ { k } } { \sqrt { \pi } } A _ { i } \left[\left(\sum_{j=1}^{m_{i}}\left[-\frac{\alpha_{e} z_{0 i j} w_{0 i j}}{\eta_{e}}\right]^{d_{0 i j}}\left[\frac{-1+z_{i j}^{2}-\left(\alpha_{e}-1\right) z_{i j} w_{i j}}{\eta_{e}}\right]^{1-d_{0 i j}-d_{2 i j}}\left[\frac{h_{2 i j}}{\eta_{e}}\right]^{d_{2 i j}}\right)\right.\right. \\
& \left(\sum_{j=1}^{m_{i}}\left[-\frac{\alpha_{e} w_{0 i j} \sqrt{2} u_{k}}{\eta_{e}}\right]^{d_{0 i j}}\left[\frac{\left(z_{i j}-\left(\alpha_{e}-1\right) w_{i j}\right) \sqrt{2} u_{k}}{\eta_{e}}\right]^{1-d_{0 i j}-d_{2 i j}}\left[\frac{h_{2 i j} \sqrt{2} u_{k}}{\eta_{e}}\right]^{d_{2 i j}}\right) \\
& +\sum_{j=1}^{m_{i}}\left[-\frac{\alpha_{e}\left(z_{0 i j}^{2}-1+w_{0 i j} z_{0 i j}\right) w_{0 i j} \sqrt{2} u_{k}}{\eta_{e}^{2}}\right]^{d_{0 i j}}\left[\frac{\left(-2 z_{i j}-\left(\alpha_{e}-1\right)\left(z_{i j}^{2} w_{i j}-w_{i j}+z_{i j} w_{i j}^{2}\right)\right) \sqrt{2} u_{k}}{\eta_{e}^{2}}\right]^{1-d_{0 i j}-d_{2 i j}} \\
& \left.\left[\frac{\left(z_{2 i j}^{2}-\left(\alpha_{e}-1\right) z_{2 i j} w_{2 i j}-1-z_{2 i j} h_{2 i j}\right) h_{2 i j} \sqrt{2} u_{k}}{\eta_{e}^{2}}\right]^{d_{2 i j}}\right] \\
& -\frac{1}{B_{i}^{2}}\left(\sum_{k=1}^{Q} \frac{\omega_{k}}{\sqrt{\pi}} A_{i} \sum_{j=1}^{m_{i}}\left[-\frac{\alpha_{e} z_{0 i j} w_{0 i j}}{\eta_{e}}\right]^{d_{0 i j}}\left[\frac{-1+z_{i j}^{2}-\left(\alpha_{e}-1\right) z_{i j} w_{i j}}{\eta_{e}}\right]^{1-d_{0 i j}-d_{2 i j}}\left[\frac{h_{2 i j}}{\eta_{e}}\right]^{d_{2 i j}}\right) \\
& \left.\left(\sum_{k=1}^{Q} \frac{\omega_{k}}{\sqrt{\pi}} A_{i} \sum_{j=1}^{m_{i}}\left[-\frac{\alpha_{e} w_{0 i j} \sqrt{2} u_{k}}{\eta_{e}}\right]^{d_{0 i j}}\left[\frac{\left(z_{i j}-\left(\alpha_{e}-1\right) w_{i j}\right) \sqrt{2} u_{k}}{\eta_{e}}\right]^{1-d_{0 i j}-d_{2 i j}}\left[\frac{h_{2 i j} \sqrt{2} u_{k}}{\eta_{e}}\right]^{d_{2 i j}}\right)\right\} \\
& j_{\eta_{e} \alpha_{e}}=-\sum_{i=1}^{n}\left\{\frac { 1 } { B _ { i } } \sum _ { k = 1 } ^ { Q } \frac { \omega _ { k } } { \sqrt { \pi } } A _ { i } \left[\left(\sum_{j=1}^{m_{i}}\left[-\frac{\alpha_{e} z_{0 i j} w_{0 i j}}{\eta_{e}}\right]^{d_{0 i j}}\left[\frac{-1+z_{i j}^{2}-\left(\alpha_{e}-1\right) z_{i j} w_{i j}}{\eta_{e}}\right]^{1-d_{0 i j}-d_{2 i j}}\left[\frac{h_{2 i j}}{\eta_{e}}\right]^{d_{2 i j}}\right)\right.\right. \\
& \left(\sum_{j=1}^{m_{i}}\left[\log \Phi\left(z_{0 i j}\right)\right]^{d_{0 i j}}\left[\frac{1}{\alpha_{e}}+\log \Phi\left(z_{i j}\right)\right]^{1-d_{0 i j}-d_{2 i j}}\left[-\frac{h_{2 i j} \log \Phi\left(z_{2 i j}\right)}{\alpha_{e} w_{2 i j}}\right]^{d_{2 i j}}\right) \\
& +\sum_{j=1}^{m_{i}}\left[-\frac{w_{0 i j} z_{0 i j}}{\eta_{e}}\right]^{d_{0 i j}}\left[-\frac{w_{i j} z_{i j}}{\eta_{e}}\right]^{1-d_{0 i j}-d_{2 i j}} \\
& \left.\left[\frac{\left(h_{2 i j}\left(\alpha_{e}^{-1}+\log \Phi\left(z_{2 i j}\right)\right)+\alpha_{e}^{-1} w_{2 i j}^{-1} h_{2 i j} \log \Phi\left(z_{2 i j}\right)\right) z_{i j}}{\eta_{e}}\right]^{d_{2 i j}}\right] \\
& -\frac{1}{B_{i}^{2}}\left(\sum_{k=1}^{Q} \frac{\omega_{k}}{\sqrt{\pi}} A_{i} \sum_{j=1}^{m_{i}}\left[-\frac{\alpha_{e} z_{0 i j} w_{0 i j}}{\eta_{e}}\right]^{d_{0 i j}}\left[\frac{-1+z_{i j}^{2}-\left(\alpha_{e}-1\right) z_{i j} w_{i j}}{\eta_{e}}\right]^{1-d_{0 i j}-d_{2 i j}}\left[\frac{h_{2 i j}}{\eta_{e}}\right]^{d_{2 i j}}\right) \\
& \left.\left(\sum_{k=1}^{Q} \frac{\omega_{k}}{\sqrt{\pi}} A_{i} \sum_{j=1}^{m_{i}}\left[\log \Phi\left(z_{0 i j}\right)\right]^{d_{0 i j}}\left[\frac{1}{\alpha_{e}}+\log \Phi\left(z_{i j}\right)\right]^{1-d_{0 i j}-d_{2 i j}}\left[-\frac{h_{2 i j} \log \Phi\left(z_{2 i j}\right)}{\alpha_{e} w_{2 i j}}\right]^{d_{2 i j}}\right)\right\}
\end{aligned}
$$




$$
\begin{aligned}
& j_{\eta_{e} \alpha_{g}}=-\sum_{i=1}^{n}\left\{\frac { 1 } { B _ { i } } \sum _ { k = 1 } ^ { Q } \frac { \omega _ { k } } { \sqrt { \pi } } A _ { i } \left[\left(\sum_{j=1}^{m_{i}}\left[-\frac{\alpha_{e} z_{0 i j} w_{0 i j}}{\eta_{e}}\right]^{d_{0 i j}}\left[\frac{-1+z_{i j}^{2}-\left(\alpha_{e}-1\right) z_{i j} w_{i j}}{\eta_{e}}\right]^{1-d_{0 i j}-d_{2 i j}}\left[\frac{h_{2 i j}}{\eta_{e}}\right]^{d_{2 i j}}\right)\right.\right. \\
& \left.\left(\frac{1}{\alpha_{g}}+\log \Phi\left(\sqrt{2} u_{k}\right)\right)\right] \\
& -\frac{1}{B_{i}^{2}}\left(\sum_{k=1}^{Q} \frac{\omega_{k}}{\sqrt{\pi}} A_{i} \sum_{j=1}^{m_{i}}\left[-\frac{\alpha_{e} z_{0 i j} w_{0 i j}}{\eta_{e}}\right]^{d_{0 i j}}\left[\frac{-1+z_{i j}^{2}-\left(\alpha_{e}-1\right) z_{i j} w_{i j}}{\eta_{e}}\right]^{1-d_{0 i j}-d_{2 i j}}\left[\frac{h_{2 i j}}{\eta_{e}}\right]^{d_{2 i j}}\right) \\
& \left.\left(\sum_{k=1}^{Q} \frac{\omega_{k}}{\sqrt{\pi}} A_{i}\left[\frac{1}{\alpha_{g}}+\log \Phi\left(\sqrt{2} u_{k}\right)\right]\right)\right\} \\
& j_{\eta_{g} \eta_{g}}=-\sum_{i=1}^{n}\left\{\frac { 1 } { B _ { i } } \sum _ { k = 1 } ^ { Q } \frac { \omega _ { k } } { \sqrt { \pi } } A _ { i } \left[\left(\sum_{j=1}^{m_{i}}\left[-\frac{\alpha_{e} w_{0 i j} \sqrt{2} u_{k}}{\eta_{e}}\right]^{d_{0 i j}}\left[\frac{\left(z_{i j}-\left(\alpha_{e}-1\right) w_{i j}\right) \sqrt{2} u_{k}}{\eta_{e}}\right]^{1-d_{0 i j}-d_{2 i j}}\left[\frac{h_{2 i j} \sqrt{2} u_{k}}{\eta_{e}}\right]^{d_{2 i j}}\right)^{2}\right.\right. \\
& +\sum_{j=1}^{m_{i}}\left[-\frac{\alpha_{e}\left(z_{0 i j}+w_{0 i j}\right) w_{0 i j} 2 u_{k}^{2}}{\eta_{e}^{2}}\right]^{d_{0 i j}}\left[-\frac{\left(1+\left(\alpha_{e}-1\right)\left(z_{i j}+w_{i j}\right) w_{i j}\right) 2 u_{k}^{2}}{\eta_{e}^{2}}\right]^{1-d_{0 i j}-d_{2 i j}} \\
& \left.\left[\frac{\left(z_{2 i j}-\left(\alpha_{e}-1\right) w_{2 i j}-h_{2 i j}\right) h_{2 i j} 2 u_{k}^{2}}{\eta_{e}^{2}}\right]^{d_{2 i j}}\right] \\
& \left.-\frac{1}{B_{i}^{2}}\left(\sum_{k=1}^{Q} \frac{\omega_{k}}{\sqrt{\pi}} A_{i} \sum_{j=1}^{m_{i}}\left[-\frac{\alpha_{e} w_{0 i j} \sqrt{2} u_{k}}{\eta_{e}}\right]^{d_{0 i j}}\left[\frac{\left(z_{i j}-\left(\alpha_{e}-1\right) w_{i j}\right) \sqrt{2} u_{k}}{\eta_{e}}\right]^{1-d_{0 i j}-d_{2 i j}}\left[\frac{h_{2 i j} \sqrt{2} u_{k}}{\eta_{e}}\right]^{d_{2 i j}}\right)^{2}\right\} \\
& j_{\eta_{g} \alpha_{e}}=-\sum_{i=1}^{n}\left\{\frac { 1 } { B _ { i } } \sum _ { k = 1 } ^ { Q } \frac { \omega _ { k } } { \sqrt { \pi } } A _ { i } \left[\left(\sum_{j=1}^{m_{i}}\left[-\frac{\alpha_{e} w_{0 i j} \sqrt{2} u_{k}}{\eta_{e}}\right]^{d_{0 i j}}\left[\frac{\left(z_{i j}-\left(\alpha_{e}-1\right) w_{i j}\right) \sqrt{2} u_{k}}{\eta_{e}}\right]^{1-d_{0 i j}-d d_{2 i j}}\left[\frac{h_{2 i j} \sqrt{2} u_{k}}{\eta_{e}}\right]^{d_{2 i j}}\right)\right.\right. \\
& \left(\sum_{j=1}^{m_{i}}\left[\log \Phi\left(z_{0 i j}\right)\right]^{d_{0 i j}}\left[\frac{1}{\alpha_{e}}+\log \Phi\left(z_{i j}\right)\right]^{1-d_{0 i j}-d_{2 i j}}\left[-\frac{h_{2 i j} \log \Phi\left(z_{2 i j}\right)}{\alpha_{e} w_{2 i j}}\right]^{d_{2 i j}}\right) \\
& +\sum_{j=1}^{m_{i}}\left[-\frac{w_{0 i j} \sqrt{2} u_{k}}{\eta_{e}}\right]^{d_{0 i j}}\left[-\frac{w_{i j} \sqrt{2} u_{k}}{\eta_{e}}\right]^{1-d_{0 i j}-d_{2 i j}} \\
& \left.\left[\frac{\left(h_{2 i j}\left(\alpha_{e}^{-1}+\log \Phi\left(z_{2 i j}\right)\right)+\alpha_{e}^{-1} w_{2 i j}^{-1} h_{2 i j} \log \Phi\left(z_{2 i j}\right)\right) \sqrt{2} u_{k}}{\eta_{e}}\right]^{d_{2 i j}}\right] \\
& -\frac{1}{B_{i}^{2}}\left(\sum_{k=1}^{Q} \frac{\omega_{k}}{\sqrt{\pi}} A_{i} \sum_{j=1}^{m_{i}}\left[-\frac{\alpha_{e} w_{0 i j} \sqrt{2} u_{k}}{\eta_{e}}\right]^{d_{0 i j}}\left[\frac{\left(z_{i j}-\left(\alpha_{e}-1\right) w_{i j}\right) \sqrt{2} u_{k}}{\eta_{e}}\right]^{1-d_{0 i j}-d_{2 i j}}\left[\frac{h_{2 i j} \sqrt{2} u_{k}}{\eta_{e}}\right]^{d_{2 i j}}\right) \\
& \left.\left(\sum_{k=1}^{Q} \frac{\omega_{k}}{\sqrt{\pi}} A_{i} \sum_{j=1}^{m_{i}}\left[\log \Phi\left(z_{0 i j}\right)\right]^{I_{0 i j}}\left[\frac{1}{\alpha_{e}}+\log \Phi\left(z_{i j}\right)\right]^{1-d_{0 i j}-d_{2 i j}}\left[-\frac{h_{2 i j} \log \Phi\left(z_{2 i j}\right)}{\alpha_{e} w_{2 i j}}\right]^{d_{2 i j}}\right)\right\}
\end{aligned}
$$




$$
\begin{aligned}
& j_{\eta_{g} \alpha_{g}}=-\sum_{i=1}^{n}\left\{\frac { 1 } { B _ { i } } \sum _ { k = 1 } ^ { Q } \frac { \omega _ { k } } { \sqrt { \pi } } A _ { i } \left[\left(\sum_{j=1}^{m_{i}}\left[-\frac{\alpha_{e} w_{0 i j} \sqrt{2} u_{k}}{\eta_{e}}\right]^{d_{0 i j}}\left[\frac{\left(z_{i j}-\left(\alpha_{e}-1\right) w_{i j}\right) \sqrt{2} u_{k}}{\eta_{e}}\right]^{1-d_{0 i j}-d_{2 i j}}\left[\frac{h_{2 i j} \sqrt{2} u_{k}}{\eta_{e}}\right]^{d_{2 i j}}\right)\right.\right. \\
& \left.\left(\frac{1}{\alpha_{g}}+\log \Phi\left(\sqrt{2} u_{k}\right)\right)\right] \\
& -\frac{1}{B_{i}^{2}}\left(\sum_{k=1}^{Q} \frac{\omega_{k}}{\sqrt{\pi}} A_{i} \sum_{j=1}^{m_{i}}\left[-\frac{\alpha_{e} w_{0 i j} \sqrt{2} u_{k}}{\eta_{e}}\right]^{d_{0 i j}}\left[\frac{\left(z_{i j}-\left(\alpha_{e}-1\right) w_{i j}\right) \sqrt{2} u_{k}}{\eta_{e}}\right]^{1-d_{0 i j}-d_{2 i j}}\left[\frac{h_{2 i j} \sqrt{2} u_{k}}{\eta_{e}}\right]^{d_{2 i j}}\right) \\
& \left.\left(\sum_{k=1}^{Q} \frac{\omega_{k}}{\sqrt{\pi}} A_{i}\left[\frac{1}{\alpha_{g}}+\log \Phi\left(\sqrt{2} u_{k}\right)\right]\right)\right\} \\
& j_{\alpha_{e} \alpha_{e}}=-\sum_{i=1}^{n}\left\{\frac { 1 } { B _ { i } } \sum _ { k = 1 } ^ { Q } \frac { \omega _ { k } } { \sqrt { \pi } } A _ { i } \left[\left(\sum_{j=1}^{m_{i}}\left[\log \Phi\left(z_{0 i j}\right)\right]^{d_{0 i j}}\left[\frac{1}{\alpha_{e}}+\log \Phi\left(z_{i j}\right)\right]^{1-d_{0 i j}-d_{2 i j}}\left[-\frac{h_{2 i j} \log \Phi\left(z_{2 i j}\right)}{\alpha_{e} w_{2 i j}}\right]^{I_{2 i j}}\right)^{2}\right.\right. \\
& \left.+\sum_{j=1}^{m_{i}}\left[-\frac{1}{\alpha_{e}}\right]^{1-d_{0 i j}-d_{2 i j}}\left[-\frac{h_{2 i j} \log \Phi\left(z_{2 i j}\right)}{\alpha_{e} w_{2 i j}\left(1-\left\{\Phi\left(z_{2 i j}\right)\right\}^{\alpha_{e}}\right)}\right]^{I_{2 i j}}\right] \\
& \left.-\frac{1}{B_{i}^{2}}\left(\sum_{k=1}^{Q} \frac{\omega_{k}}{\sqrt{\pi}} A_{i} \sum_{j=1}^{m_{i}}\left[\log \Phi\left(z_{0 i j}\right)\right]^{d_{0 i j}}\left[\frac{1}{\alpha_{e}}+\log \Phi\left(z_{i j}\right)\right]^{1-d_{0 i j}-d_{2 i j}}\left[-\frac{h_{2 i j} \log \Phi\left(z_{2 i j}\right)}{\alpha_{e} w_{2 i j}}\right]^{d_{2 i j}}\right)^{2}\right\} \\
& j_{\alpha_{e} \alpha_{g}}=-\sum_{i=1}^{n}\left\{\frac { 1 } { B _ { i } } \sum _ { k = 1 } ^ { Q } \frac { \omega _ { k } } { \sqrt { \pi } } A _ { i } \left[\left(\sum_{j=1}^{m_{i}}\left[\log \Phi\left(z_{0 i j}\right)\right]^{d_{0 i j}}\left[\frac{1}{\alpha_{e n}}+\log \Phi\left(z_{i j}\right)\right]^{1-d_{0 i j}-d_{2 i j}}\left[-\frac{h_{2 i j} \log \Phi\left(z_{2 i j}\right)}{\alpha_{e} w_{2 i j}}\right]^{I_{2 i j}}\right)\right.\right. \\
& \left.\left(\frac{1}{\alpha_{g}}+\log \Phi\left(\sqrt{2} u_{k}\right)\right)\right] \\
& -\frac{1}{B_{i}^{2}}\left(\sum_{k=1}^{Q} \frac{\omega_{k}}{\sqrt{\pi}} A_{i} \sum_{j=1}^{m_{i}}\left[\log \Phi\left(z_{0 i j}\right)\right]^{d_{0 i j}}\left[\frac{1}{\alpha_{e}}+\log \Phi\left(z_{i j}\right)\right]^{1-d_{0 i j}-d_{2 i j}}\left[-\frac{h_{2 i j} \log \Phi\left(z_{2 i j}\right)}{\alpha_{e} w_{2 i j}}\right]^{I_{2 i j}}\right) \\
& \left.\left(\sum_{k=1}^{Q} \frac{\omega_{k}}{\sqrt{\pi}} A_{i}\left[\frac{1}{\alpha_{g}}+\log \Phi\left(\sqrt{2} u_{k}\right)\right]\right)\right\} \\
& j_{\alpha_{g} \alpha_{g}}=-\sum_{i=1}^{n}\left\{\frac{1}{B_{i}} \sum_{k=1}^{Q} \frac{\omega_{k}}{\sqrt{\pi}} A_{i}\left[\left(\frac{1}{\alpha_{g}}+\log \Phi\left(\sqrt{2} u_{k}\right)\right)^{2}-\frac{1}{\alpha_{g}^{2}}\right]\right. \\
& \left.-\frac{1}{B_{i}^{2}}\left(\sum_{k=1}^{Q} \frac{\omega_{k}}{\sqrt{\pi}} A_{i}\left[\frac{1}{\alpha_{g}}+\log \Phi\left(\sqrt{2} u_{k}\right)\right]\right)^{2}\right\}
\end{aligned}
$$




\section{Referências Bibliográficas}

Aitkin (1999) M. Aitkin. A general maximum likelihood analysis of variance components in generalized linear models. Biometrics, 55:117-128. Citado na pág. 1

Akaike (1974) H. Akaike. A new look at the statistical model identification. Autom Control IEEE Trans, 19:716-723. Citado na pág. 26, 35

Arellano-Valle e Azzalini (2008) R. B. Arellano-Valle e A. Azzalini. The centred parametrization for the multivariate skew-normal distribution. Journal of Multivariate Analysis., 99 (7):1362-1382. doi: 10.1016/j.jmva.2008.01.020. Citado na pág. 6, 7, 24

Arellano-Valle e Azzalini (2013) R. B. Arellano-Valle e A. Azzalini. The centred parameterization and related quantities of the skew- $t$ distribution. Journal of Multivariate Analysis., 113:73-90. doi: 10.1016/j.jmva.2011.05.016. Citado na pág. 24, 25

Arellano-Valle et al. (2005) R. B. Arellano-Valle, H. Bolfarine e V. H. Lachos. Skew-normal linear mixed models. Journal of Data Science, 3(4):415-438. Citado na pág. 41, 42, 48, 52, 56

Arellano-Valle et al. (2007) R. B. Arellano-Valle, H. Bolfarine e V. H. Lachos. Bayesian inference for skew-normal linear mixed models. Journal of Applied Statistics, 34(6):663-682. Citado na pág. 41

Arellano-Valle et al. (2012) R. B. Arellano-Valle, L. M. Castro, G. González-Farías e K. A. Munõz Gajardo. Student- $t$ censored regression model: properties and inference. Statistical Methods $\mathscr{E}$ Applications, 21(4):453-473. doi: 10.1007/s10260-012-0199-y. Citado na pág. iii, iv, $4,16,29,32,35,36,40,81$

Arnold (2004) B.C. Arnold. Discussion of jones, m. c. families of distribtion arising from distribution of order statistics. Test, 13:23-25. Citado na pág. 9

Arnold et al. (1999) B.C. Arnold, E. Castillo e J. M. Sarabia. Conditionally Specification of Statistical Model. Springer Series in Statistics, Springer-Verlag, New York. Citado na pág. 12

Atkinson (1985) A. C. Atkinson. Plots, Transformations and Regression: An Introduction to Graphical Methods of Diagnostic Regression Analysis. Oxford University Press", New York. Citado na pág. 46, 48

Azzalini (1985) A. Azzalini. A class of distributions which includes the the normal ones. Scandinavian Journal of Statistics, 12:171-178. Citado na pág. 6, 9, 10, 16, 78

Azzalini e Capitanio (2003) A. Azzalini e A. Capitanio. Distributions generated by perturbation of symmetry with emphasis on a multivariate skew $t$-distribution. Journal of the Royal Statistical Society. Series B (Statistical Methodology), 65(2):367-389. doi: 10.1111/1467-9868.00391. Citado na pág. iii, iv, 6, 8, 10, 16, 17, 39 
Barros et al. (2010) M. Barros, M. Galea, M. González e V. Leiva. Influence diagnostics in the tobit censored response model. Statistical Methods \& Applications, 19(3):379-397. doi: 10.1007/s10260-010-0135-y. Citado na pág. 35

Bayes et al. (2012) C. L. Bayes, J. L. Bazán e C García. A new robust regression model for proportions. Bayesian Analysis, 7(4):841-866. Citado na pág. 1

Bozdogan (1987) H. Bozdogan. Model selection and akaike's information criterion (AIC): The general theory and its analytical extensions. Psychometrika., 52(3):345-370. doi: 10.1007/ BF02294361. Citado na pág. 26

Branco e Dey (2001) M. D. Branco e D. K. Dey. A general class of multivariate skew-elliptical distributions. Journal of Multivariate Analysis, 79(1):99-113. doi: 10.1006/jmva.2000.1960. Citado na pág. 8, 16

Branscum et al. (2007) A. J Branscum, W. O Johnson e M. C. Thurmond. Bayesian beta regression: Applications to household expenditure data and genetic distance between foot and mouth disease viruses. Australian and New Zealand Journal of Statistics, 49(3):287-301. Citado na pág. 1

Butler e Louis (1992) S. M. Butler e T. A Louis. Random effects models with non parametrics priors. Satatistics in Medicine, 111:1981-2000. Citado na pág. 41

Chen et al. (2002) J. Chen, D. Zhang e M. Davidian. A monte carlo em algorithm for generalized linear mixed models with flexible random effects distributions. Biostatistics, 3:347-360. Citado na pág. 1

Colosimo e Giolo (2006) E. A. Colosimo e S. R Giolo. Análise de Sobrevivência Aplicada. ABE-Projeto Fisher. Citado na pág. 3

Delandre et al. (2014) M. Delandre, M. Lavielle e M. A. Poursat. A note on BIC in mixedeffects models. Electronic Journal of Statistics, 8(1):456-475. doi: 10.1214/14-EJS890. Citado na pág. 46,72

Diggle et al. (2004) P. J. Diggle, P. Heagerty, Liang K. Y. e S. L. Zeger. Analysis of Longitudinal Data. Oxford University Press, Oxford, second ed. Citado na pág. 1

Durrans (1992) S. R. Durrans. Distributions of fractional order statistics in hydrology. Water Resources Research, 28:1649-1655. Citado na pág. 9, 10, 16, 42, 78

Evans e Swartz (2000) M. Evans e T. B. Swartz. Approximating Integrals via Monte Carlo and Deterministic Methods. Oxford University Press, New York, first ed. Citado na pág. 15

Fahrmeir e Tutz (2001) L. Fahrmeir e G. Tutz. Multivariate Statistical Modelling Based on Generalized Linar Models. Springer Verlag, New York, second ed. Citado na pág. 15

Fernandes et al. (2006) J. Fernandes, C. Salinas, S. London, R. Wiegand, E. Hill, E. Slate, J. Grewal, P. Werner, J. Sanders e M. Lopes-Virella. Prevalence of periodontal disease in gullah african american diabeticsm. Journal of Dental Research, 85:997. Citado na pág. 73

Ferrari e Cribari-Neto (2004) S. L. Ferrari e F. Cribari-Neto. Beta regression for modelling rates and proportion. Journal of Applied Statistics, 31:799-815. Citado na pág. 1 
Figueroa-Zúñiga et al. (2013) J. Figueroa-Zúñiga, R. Arellano-Valle e S. L. Ferrari. Mixed beta regression: A bayesian perspective. Computational Statistics and Data Analysis, 61:137147. Citado na pág. 1,80

Fitzmaurice et al. (2004) G. M. Fitzmaurice, N. M. Laid e J. H. Ware. Applied Longitudinal Analysis. Wiley-Interscience, New Jersey. Citado na pág. 5

Flecher et al. (2010) C. Flecher, D. Allard e P. Naveau. Truncated skew-normal distributions: moments, estimation by weighted moments and application to climatic data. MetronInternational Journal of Statistics, 68(3):331-345. Citado na pág. 7, 8

Fong et al. (2010) Y. Fong, H. Rue e J. Wakefield. Bayesian inference for generalized linear mixed models. Biostatistics, 11(3):397-412. Citado na pág. 1

Gallant e Nychka (1987) A. R. Gallant e D. W. Nychka. Semi-nonparametric maximum likelihood estimation. Econometrica, 55(2):363-390. doi: 10.2307/1913241. Citado na pág. 41

Galvis et al. (2014) D. M. Galvis, D. Bandyopadhyay e V. H.. Lachos. Augmented mixed beta regression models for periodontal proportion data. Statistics in Medicine, 33(21):3759-3771. doi: 10.1002/sim.6179. Citado na pág. 1, 2, 57, 73

Garay et al. (2017) A. M. Garay, V. H. Lachos, H. Bolfarine e C. R. B Cabral. Linear censored regression models with scale mixtures of normal distributions. Statistical Papers, 58(1):247278. doi: 10.1007/s00362-015-0696-9. Citado na pág. 4, 35, 36, 37, 40, 81

Garay (2014) A. W. Garay. Modelos de regressão para dados Censurados sob Distribuições Simétricas. Tese de Doutorado, Instituto de Matemática e Estatística, Universidade de São Paulo, Brasil. Citado na pág. 3

Global Volcanism Program (2013) Global Volcanism Program. Volcanoes of the world, v. 4.6.0. venzke, e (ed.). Smithsonian Institution. doi: dx.doi.org/10.5479/si.GVP.VOTW4-2013. Citado na pág. 25

Gupta (2003) A. K. Gupta. Multivariate skew t-distribution. Statistics, 37(4):359-363. doi: 10.1080/715019247. Citado na pág. 16

Gupta e Gupta (2004) R. C. Gupta e R. D. Gupta. Generalized skew normal model. Sociedad de Estad stica e Im estigacidn Operativa, 13(2):501-524. Citado na pág. 10

Gupta e Gupta (2008) R. D. Gupta e R. C. Gupta. Analyzing skewed data by power normal model. Test, 17(1):197-210. doi: 10.1007/s11749-006-0030-x. Citado na pág. 1, 8, 9, 10, 81

Hughes (1999) J. P. Hughes. Mixed effects models with censored data with application to hiv rna levels. Biometric, 55:625-629. Citado na pág. 1

Jacqmin-Gadda et al. (2000) H. Jacqmin-Gadda, R. Thiébaut, G. Chêne e D Commenges. Analysis of left-censored longitudinal data with application to viral load in hiv infection. Bioestatistics, 1(4):397-412. Citado na pág. 1

Jones e Faddy (2003) M. C. Jones e M. J. Faddy. A skew extension of the $t$-distribution, with applications. Journal of the Royal Statistical Society. Series B (Statistical Methodology), 65 (1):159-174. Citado na pág. 16

Kalbfleish e Lawless (1992) J. Kalbfleish e J. Lawless. Some useful statistical methods for truncated data. Journal of Quality and Technology, 24:145-152. Citado na pág. 4 
Kundu e Gupta (2013) D Kundu e R. D. Gupta. Power-normal distribution. Statistics, 47 (1):110-125. doi: 10.1080/02331888.2011.568620. Citado na pág. 10, 12, 13, 14

Kutner et al. (2005) M. Kutner, C. J. Nachtsheim, J. Neter e W. Li. Applied Linear statistical Models. McGraw-Hill, New York, fifth ed. Citado na pág. 46, 48

Lachos (2004) V. H. Lachos. Modelos lineares mistos assimetricos. Tese de Doutorado, Instituto de Matemática e Estatística, Universidade de São Paulo, Brasil. Citado na pág. 41

Lachos et al. (2010) V. H. Lachos, P. Ghosh e R. B. Arellano-Valle. Likelihood based inference for skew-normal independent linear mixed models. Statistica Sinica, 20(1):303-322. Citado na pág. 41

Laird e Ware (1982) N. M. Laird e J. H. Ware. Random-effects models for longitudinal data. Biometricss, 38(4):963-974. Citado na pág. 2, 4, 5

Lee e Thompson (2008) K. J. Lee e S. G. Thompson. Flexible parametric models for randomeffects distributions. Statistics in Medicine, 27:418-43. Citado na pág. 1

Lehmann (1953) E. L. Lehmann. The power of rank tests. Ann Math Stat, 24:23-43. Citado na pág. 10

Lin e Lee (2008) T. I. Lin e J. K. Lee. Estimation and prediction in linear mixed models with skew-normal random effects for longitudinal data. Statistics in Medicine, 27(9):1490-1507. doi: 10.1002/sim.3026. Citado na pág. 41

Lindstrom e Bates (1988) M. J. Lindstrom e D. M. Bates. Newton-raphson and em algorithms for linear mixed-effects models for repeated-measures data. Journal of the American Statistical Association, 83(404):1014-1022. Citado na pág. 5

Martínez-Flórez et al. (2013a) G. Martínez-Flórez, B. C. Arnold, H. Bolfarine e H. W. Gómez. The multivariate alpha-power model. Journal of Statistical Planning and Inference, 143(7): 1244-1255. doi: 10.1016/j.jspi.2013.01.008. Citado na pág. 10, 12

Martínez-Flórez et al. (2013b) G. Martínez-Flórez, H. Bolfarine e H. Gómez. The alphapower tobit model. Communications in Statistics - Theory and Methods, 42(4):633-643. doi: 10.1080/03610926.2011.630770. Citado na pág. 1, 32, 33, 63

Martínez-Flórez et al. (2014) G. Martínez-Flórez, H. Bolfarine e H. W. Gómez. Skew-normal alpha-power model. Statistic: A journal of Theoretical and Applied Statistics, 48(6):1414-1428. doi: 10.1080/02331888.2013.826659. Citado na pág. 16, 17, 21

Martínez-Flórez et al. (2015) G. Martínez-Flórez, H. Bolfarine e H. W. Gómez. Doubly censored power-normal regression models with inflation. Test, 24(2):265-286. Citado na pág. 57

Matos et al. (2013) L. A. Matos, M. O. Pretes, M. H. Chen e V. H. Lachos. Likelihood-based inference for mixed-effects models with censored response using the multivariate- $t$ distribution. Statistica Sinica, 23:1323-1342. Citado na pág. 4

McCulloch et al. (2008) C. E. McCulloch, S. R. Searle e J. M. Neuhaus. Generalized, Linear, and Mixed Models. John Wiley and Sons, New York, second ed. Citado na pág. 1

Molenberghs e Verbeke (2005) G. Molenberghs e G. Verbeke. Models for Discrete Longitudinal Data. Springer Verlag, New York. Citado na pág. 1, 6, 46 
Moulton e Hasley (1995) L. H. Moulton e N. A. Hasley. A mixture model with detection limits for regression analyses of antibody response to vaccine. Biometrics, 51:1570-1578. Citado na pág. 57

Mroz (1987) T. A. Mroz. The sensitivity of an empirical model of married women's hours of work to economic and statistical assumptions. Econometrica, 55(4):765-799. doi: 10.2307/ 1911029. Citado na pág. 36, 37

Nelson (1977) F. D Nelson. Censored regression models with unobserved, stochastic censoring thresholds. Journal of Econometrics, 6:309-327. Citado na pág. 4

Nelson (1990) W. Nelson. Hazard plotting of left truncated life data. Journal of Quality and Technology, 22:230-238. Citado na pág. 4

Olsen (1978) R. J. Olsen. Note on the uniqueness of the maximum likelihood estimator for the tobit model. Econometrica, 46(5):1211-1215. Citado na pág. 29, 30, 33

Ortega et al. (2003) E. M. Ortega, H. Bolfarine e G. A. Paula. Influence diagnostics in generalized log-gamma regression models. Computational Statistics and Data Analysis, 42: 165-186. Citado na pág. 35

Pan e Louis (2000) W. Pan e T. A Louis. Mixed-effects model for multivariate censored data. Biometrics, 56(1):160-166. Citado na pág. 1

Park et al. (2007) J. W. Park, M. G. Genton e S. K. Ghosh. Censored times series analysis with autoregressive moving average models. Canadian Journal of Statistics, 35:151-168. Citado na pág. 4

Pewsey et al. (2012) A. Pewsey, H. W. Gómez e H. Bolfarine. Likelihood-based inference for power distributions. Test, 21(4):775-789. doi: 10.1007/s11749-011-0280-0. Citado na pág. 8, 10

Pinheiro e Bates (2000) J. C. Pinheiro e D. M. Bates. Mixed-Effects Models in S and S-PLUS. Springer Verlag, New York. Citado na pág. 5

Pinheiro e Chao (2006) J. C. Pinheiro e E. C. Chao. Efficient laplacian and adaptative gaussian quadrature algorithms for multilevel generalized linear mixed models. American Statistical Association, 15(1):58-81. Citado na pág. 44

Pinheiro et al. (2001) J. C. Pinheiro, C. Liu e Y. N. Wu. Efficient algorithms for robust estimation in linear mixed-effects models using the multivariate $t$ distribution. Journal of Computational and Graphical Statistics, 10(2):249-276. doi: 10.1198/10618600152628059. Citado na pág. 41

R Development Core Team (2017) R Development Core Team. R: A language and environment for statistical computing. Vienna, Austria. R Foundation for Statistical Computing. URL http://www.R-project.org. ISBN 3-900051-07-0. Citado na pág. 18, 26, 52, 53, 73, 75, 78

Rocha (2004) F. M. Rocha. Seleção de estruturas de covariância em dados com medidas repetidas. dissertação de mestrado. Dissertação de Mestrado, Instituto de Matemática e Estatística, Universidade de São Paulo, Brasil. Citado na pág. 5

Rosa et al. (2003) G. J. M Rosa, C.R. Padovani, e G. Gianola. Robust linear mixed models with normal/independent distributions and bayesian MCMC implementation. Biometrical Journal, 45(5):573-590. doi: 10.1002/bimj.200390034. Citado na pág. 41 
Sahu et al. (2003) S. K. Sahu, D. K. Dey e M. D Branco. A new class of multivariate skew distributions with applications to bayesian regression models. The Canadian Journal of Statisticst, 31(2):129-150. doi: 10.2307/3316064. Citado na pág. 16

Schwarz (1978) G. Schwarz. Estimating the dimension of a model. The Annals of Statistics, 6(2):461-464. Citado na pág. 26, 35

Stapleton e Young (1984) D. C. Stapleton e J. D. Young. Censored normal regression with measurement error on the dependent variable. Econometrica, páginas 737-760. Citado na pág. 4

Sugiura (1978) N. Sugiura. Further analysis of the data by akaike's information criterion and the finite corrections. Communications In Statistics - Theory And Methods, 7(1):13-26. doi: 10.1080/03610927808827599. Citado na pág. 35

Thompson e Nelson (2003) M. L. Thompson e K. P. Nelson. Linear regression with Type I interval and left-censored responde data. Environmental and Ecological Statistics, 10:221-230. Citado na pág. 4

Tobin (1958) J. Tobin. Estimation of relationships for limited dependent variables. Econometrica, 26(1):25-36. Citado na pág. 1, 16, 32, 57, 63, 80

Usuga (2013) O. C. Usuga. Modelos de regressão beta com efeitos aleatórios normais e não normais para dados longitudinais. Tese de Doutorado, Instituto de Matemática e Estatística, Universidade de São Paulo, Brasil. Citado na pág. 46, 47

Vaida e Blanchard (2005) F. Vaida e S. Blanchard. Conditional akaike information for mixedeffects models. Biometrika, 92:351-370. doi: 10.1093/biomet/92.2.351. Citado na pág. 46, 72

Vaida e Liu (2009) F. Vaida e L. Liu. Fast implementation for normal mixed effects models with censored response. Journal of Computational and Graphical Statistics, 18:797-817. Citado na pág. 4

Vaida et al. (2007) F. Vaida, A. Fitzgerald e V. DeGruttola. Efficient hybrid em for linear and nonlinear mixed effects models with censored response. Computational Statistics and Data Analysis, 51(12):5718-5730. Citado na pág. 44

Verbeke e Lasaffre (1997) G. Verbeke e E. Lasaffre. The effects of misspecifying the randomeffects distribution in linear mixed models for longitudinal data. Computational Statistics and Data Analysis, 23:541-556. Citado na pág. 1, 41

Verbeke e Molenberghs (2009) G. Verbeke e G. Molenberghs. Linear Mixed Models for Longitudinal Data. Springer Verlag, New York, second ed. Citado na pág. 1, 4, 6

Verbeke et al. (2001) G. Verbeke, B. Spiessens e E. Lassafre. Condicional linear mixed models. The American Statistician, 55(1):25-34. Citado na pág. 4, 6

Vock et al. (2012) D. M. Vock, M. Davidiam, A. A. Tsiatis e A. J. Muir. Mixed model analysis of censored longitudinal data with flexible random-effects density. Biostatistics, 13(1):61-73. Citado na pág. 1

Weiss (2005) R. E. Weiss. Modeling Longitudinal Data. Springer Verlag, New York. Citado na pág. 5 
Wu (2002) L. Wu. A joint model for nonlinear mixed-effects models with censoring and covariates measured with error, with application to aids studies. Journal of the American Statistical Association, 97(460):955-964. Citado na pág. 1

Wu (2010) L. Wu. Mixed Effects Models for Complex Data. Chapman and Hall/CRC, Florida. Citado na pág. 44

Yu (2010) B. Yu. A bayesian mcmc approach to survival analysis with doubly-censored data. Computational Statistics and Data Analysis, 54:1921-1929. Citado na pág. 2

Zhang e Davidiam (2001) D Zhang e M. Davidiam. Linear mixed models with flexible distributions of random effects for longitudinal data. BIOMETR, 57:975-802. Citado na pág. 41, 42,52

Zhao e Kim (2016) J. Zhao e H. M. Kim. Power $t$ distribution. Communications for Statistical Applications and Methods, 23(4):321-334. doi: 10.5351/CSAM.2016.23.4.321. Citado na pág. iii, iv, $2,8,10,16,17,23,39,82$ 